\title{
Clausal Relations and $C$-clones.
}

\author{
DISSERTATION
}

zur Erlangung des akademischen Grades

Doctor rerum naturalium

(Dr. rer. nat.)

vorgelegt

der Fakultät Mathematik und Naturwissenschaften der Technischen Universität Dresden

von

M.Sc. Edith Mireya Vargas García

geboren am: 19.03.1979 in: Mexiko.

Gutachter: Prof. Dr. Dr. hc. Klaus Denecke

Prof. Dr. rer. nat. habil. Reinhard Pöschel

Eingereicht am: 02.03 .2011

Tag der Disputation: 26.05.2011

Betreuer:

Prof. Dr. rer. nat. habil. R. Pöschel 



\section{Acknowledgements}

First of all I would like to express my deepest gratitude to Prof. Reinhard Pöschel, who kindly introduced me to Universal Algebra and Function Algebras during the first year of my doctoral studies, and gave me my first algebra research problem. I am very thankful to him for sharing his wisdom and for the almost endless patience with which he guided me while preparing this work. I was always impressed by his tenacity, and even though I might not meet his high standards, I hope this dissertation will not disappoint him.

I am also very grateful to Mike Behrisch, who deepened my insight of Clone theory. The discussions with him propelled greatly this work. According to his advice the results presented in Chapter 4 are a lot more elegant and readable. I am very thankful to him for his useful comments, remarks, and pointing out some weaknesses in my argumentation.

My sincere thanks to the members of the Institute of Algebra for providing a friendly and supportive research environment. I thank Heiko Reppe and Christian Zschalig for pointing out some writing errors. Especially, I thank Christian Meschke, who introduced me to lattice and order theory.

Also, I would like to thank my family; my grandmother, my mother, my brothers and sisters, and my boyfriend for their patience. Since without their sacrifices and constant support, I might not have been able to complete this dissertation.

Finally, my thanks to the CONACYT (The National Council on Science and Technology of Mexico) for the financial support that allowed me to study in Germany. 



\section{Contents}

Acknowledgements $\quad$ i

Introduction v v

1 Preliminaries 1

1.1 Clones . . . . . . . . . . . . . . . . . . . . . . 1

1.2 Relational clones . . . . . . . . . . . . . . . . . . . . . . . . . . . . .

1.3 The Galois connection $\mathrm{Pol}-\mathrm{Inv} \ldots \ldots . . \ldots . . \ldots 5$

1.4 The Constraint Satisfaction Problem . . . . . . . . . . . . . 10

2 Clausal relations $\quad \mathbf{1 1}$

2.1 Introduction . . . . . . . . . . . . . . . . . . . . . 11

2.2 Operations on clausal relations . . . . . . . . . . . . . . . 12

3 C-clones 17

3.1 Galois connection Pol $-C$ Inv . . . . . . . . . . . . . . . 17

3.2 Boolean $C$-clones . . . . . . . . . . . . . . . . . . 18

3.3 The duality principle for $C$-clones . . . . . . . . . . . . . . . 29

3.4 Some $C$-clones on a finite set. . . . . . . . . . . . . . . 33

3.5 Infinite chains of $C$-clones. . . . . . . . . . . . . . . 38

$4 C$-automorphism groups and Krasner $C$-clones $\quad 47$

4.1 Characterisation . . . . . . . . . . . . . . . . 47

$4.2 C$-automorphism groups and the partition lattice ....... 54

$5 C$-monoids and weak Krasner $C$-clones $\quad \mathbf{5 7}$

5.1 Describing $C$-monoids . . . . . . . . . . . . . . . 57

$5.2 \quad C$-monoids for $|D|=3 \ldots \ldots \ldots \ldots \ldots$

5.3 Maximal $C$-monoids . . . . . . . . . . . . . . . 66

5.4 Minimal $C$-monoids . . . . . . . . . . . . . . . . . 69

5.5 Special $C$-monoids for $|D| \geq 3 \ldots \ldots \ldots \ldots$. . . . . . . . . . . . . . .

6 Maximal $C$-clones $\quad \mathbf{8 5}$

6.1 Description . . . . . . . . . . . . . . . . . 85

$6.2 \quad C$-complete operations . . . . . . . . . . . . . . 87

7 Problems for future research $\quad 95$ 
8 Concluding remarks $\quad 99$

$\begin{array}{lr}\text { Notation } & 101\end{array}$

$\begin{array}{ll}\text { Index } & 105\end{array}$

$\begin{array}{ll}\text { References } & 107\end{array}$ 


\section{Introduction}

Systems of operations are considered in the Clone theory. Clones are sets of operations on a fixed set that are closed under composition and contain all projections. The clones on a finite set $D$ are the Galois closed sets of operations with respect to the well-known Galois connection $\mathrm{Pol}_{D}-\operatorname{Inv}_{D}$ induced by the preservation relation "an operation $f$ preserves a relation $\varrho$ " (see [PK79]). The latter will be used frequently in the subsequent chapters.

In this dissertation a special set of relations on a finite set $D$, called clausal relations (see Definition 2.1.2), is introduced. The definition of a clausal relation is based on the notion of a clausal constraint, which is a disjunction of inequalities of the form $x \geq d$ and $x \leq d$, where $d \in D=\{0,1, \ldots, n-1\}$ and $x$ belongs to a set $X$ of variables. Clausal constraints were studied by N. Creignou, M. Hermann, A. Krokhin and G. Salzer (see [CHKS08]) to classify the complexity of constraints.

The correspondence between various sets of relations and clones has been proven to be quite fruitful in computer sciences; in particular, to identify polynomialtime solvable subclasses of the Constraint Satisfaction Problem (CSP)(see [Jea98], and [BKJ00]). In this thesis it is shown that clausal relations meet a sufficient condition that is known to ensure polynomial time solvability of the corresponding CSP, namely that their clone of polymorphisms contains a constant operation (see [JCG97]). Therefore, for clausal relations the CSP is solvable in polynomial time.

The set of all clones on $D$ forms a complete lattice with respect to the set-theoretic inclusion. There are countably many clones on the two-element set, and their lattice is completely known (see [Pos41]). Furthermore, since there are continuum-many clones on a set containing at least three elements (see [PK79]), it seems to be hopeless to find a full description of this lattice. Therefore, a restriction to special relations makes sense.

Our aim is to describe clones which are determined by sets of clausal relations. These are called $C$-clones (see Definition 3.1.2). The restriction of the Galois connection $\mathrm{Pol}_{D}-\mathrm{Inv}_{D}$ to clausal relations gives rise to the Galois connection $\mathrm{Pol}_{D}-C \mathrm{Inv}_{D}$. The latter yields a smaller number of Galois closed sets of operations ( $C$-clones), a fact that motivated us to find all $C$-clones on $D$, and to describe them.

In particular, this work can be seen as a contribution to reveal the structure of the clone lattice. This dissertation is organised as follows.

First, Chapter 1 recalls basic concepts such as clone; relational, weak Krasner, and Krasner clone. Furthermore, it provides the Galois connection $\mathrm{Pol}_{D}-\mathrm{Inv}_{D}$ and the definition of the Constraint Satisfaction Problem over sets of relations. 
In Chapter 2 the set of clausal relations is defined. Some operations on clausal relations are studied. Moreover, in Section 2.2 we prove that this set can be partitioned into trivial and non-trivial clausal relations.

In Chapter 3 the lattice of $C$-clones is analysed. First, we prove that every $C$-clone is determined by a set of non-trivial clausal relations. Second, we describe the lattice of $C$-clones on the two-element set. The resulting lattice is a 5-element sublattice of the lattice described by E., L. Post (see [Pos41]). In Section 3.4 it is shown that clausal relations meet a sufficient condition that is known to ensure polynomial time solvability of the corresponding CSP. Finally, for $|D| \geq 3$ the existence of infinitely many $C$-clones is shown (see also [Var10]). Therefore, we analyse the lattice of $C$-clones up to equality of their unary bijective parts. Due to the Galois connection $\mathrm{Pol}_{D}-\operatorname{Inv}_{D}$ this is equivalent to the study of automorphism groups of clausal relations, the so-called $C$-automorphism groups.

In Chapter 4 we describe the lattice of $C$-automorphism groups. It turns out that the precondition of bijectivity imposes such strong constraints on the $C$-clone lattice that the resulting lattice is Boolean with $|D|-1$ atoms. Furthermore, it is shown that the Krasner clones (see Section 1.3) corresponding to the $C$-automorphism groups are determined by some of their unary relations, which are intervals w.r.t. the natural order on $D$.

In Chapter 5 we study the $C$-clone lattice up to equality of the corresponding endomorphism monoids, which is the lattice of $C$-monoids. The structure of this lattice is considerably harder to understand, since unary relations do not suffice to describe $C$-monoids. However, in Section 5.1, it is shown that finite sets of clausal relations suffice to describe $C$-monoids. Then, in Section 5.2 the lattice of $C$-monoids for $|D|=3$ is determined. After that, in Sections 5.3 and 5.4, all elements neighbouring the top (co-atoms) and the bottom (atoms) in the $C$-monoid lattice are characterised (see also [Var12]).

In Chapter 6 all co-atoms in the $C$-clone lattice are described. These co-atoms can be described by non-trivial binary clausal relations and it turns out that they coincide with the relations which characterise co-atoms in the lattice of $C$-monoids.

In Chapter 7 some open problems are presented that emerged while working on this dissertation.

Finally, in Chapter 8 some concluding remarks can be found. 


\section{Preliminaries}

This chapter provides definitions, notations and conventions used in the subsequent chapters. Operations, relations, clones of operations defined on a fixed set will be studied. Hence, some notational issues associated with these concepts are addressed in Section 1.1. In Section 1.2 we discuss relational clones, while in Section 1.3 the connection between sets of relations and sets of operations is explained. Finally, in Section 1.4 the definition of the Constraint Satisfaction Problem over sets of relations is given.

Throughout the text $\mathbb{N}=\{0,1,2,3, \ldots\}$ denotes the set of natural numbers, $\mathbb{N}_{+}=\{1,2, \ldots\}$ denotes the set of positive natural numbers, and $D$ (also called domain) represents the set $D=\{0,1, \ldots, n-1\}$ for a fixed natural number $n \geq 2$.

\subsection{Clones}

Let $k$ be a positive natural number. A $k$-ary operation on the domain $D$ is a function $f: D^{k} \longrightarrow D$. We denote by $\mathrm{O}_{D}^{(k)}:=\left\{f \mid f: D^{k} \longrightarrow D\right\}$ the set of all $k$-ary operations on $D$ and by

$$
\mathrm{O}_{D}:=\bigcup_{k=1}^{\infty} \mathrm{O}_{D}^{(k)}
$$

the set of all finitary operations on $D$. Moreover, for each $j \in\{1, \ldots, k\}$ let denote by $e_{j}^{k}\left(d_{1}, \ldots, d_{k}\right):=d_{j}$ the $j$-th projection of arity $k$ and by

$$
J_{D}:=\bigcup_{k=1}^{\infty}\left\{e_{j}^{k} \mid 1 \leq j \leq k\right\}
$$

the set of all projections on $D$.

The composition or superposition of a $k$-ary operation $f$ with operations $g_{1}, \ldots, g_{k}$ of arity $m$ is the $m$-ary operation $f\left(g_{1}, \ldots, g_{k}\right)$ defined by

$$
f\left(g_{1}, \ldots, g_{k}\right)\left(x_{1}, \ldots, x_{m}\right):=f\left(g_{1}\left(x_{1}, \ldots, x_{m}\right), \ldots, g_{k}\left(x_{1}, \ldots, x_{m}\right)\right)
$$

for all $x_{1}, \ldots, x_{m} \in D$.

Now, we will define a clone.

1.1.1 Definition ([Pös04]). Any set of (finitary) operations (on a fixed base set $D$ ) that is closed under composition and contains all projections is called a clone on $D$. If $F \subseteq \mathrm{O}_{D}$ is a clone, we will denote this by $F \leq \mathrm{O}_{D}$. 
Some basic examples of clones are the following:

- The set $\mathrm{O}_{D}$ is called the full clone $D$.

- The set $J_{D}$ is called the trivial clone on $D$.

- The set of all idempotent operations on $D$, i.e.

$$
I=\left\{f \in \mathrm{O}_{D}^{(k)} \mid f(x, \ldots, x)=x\right\} .
$$

- All continuous operations on a topological space.

The following handy remark permits the definition of the clone generated by a subset $F$ of $\mathrm{O}_{D}$, denoted by $\langle F\rangle_{O_{D}}$, as the intersection of all clones that contain $F$, i.e.

$$
\langle F\rangle_{O_{D}}:=\bigcap\left\{C \mid C \leq \mathrm{O}_{D} \wedge F \subseteq C\right\} .
$$

\subsubsection{Remark ([PK79]). The intersection of a set of clones on $D$ is a clone.}

Proof. Let $C_{i}$ be clones on $D$ where $i \in I$, and let $\mathcal{C}:=\bigcap_{i \in I} C_{i}$. We show that $\mathcal{C}$ is a clone. Clearly, $J_{D} \subseteq \mathcal{C}$. Let $k, m$ be positive natural numbers, $f \in \mathrm{O}_{D}^{(k)}$ and let $g_{1}, \ldots, g_{k} \in \mathrm{O}_{D}^{(m)}$. If $\left\{f, g_{1}, \ldots, g_{k}\right\} \subseteq \bigcap_{i \in I} C_{i}$, then we get $\left\{f, g_{1}, \ldots, g_{k}\right\} \subseteq C_{i}$ for all $i \in I$. Since $C_{i}$ are clones on $D$, we obtain $f\left(g_{1}, \ldots, g_{k}\right) \in C_{i}$ for all $i \in I$, and hence $f\left(g_{1}, \ldots, g_{k}\right) \in \mathcal{C}$.

It is not difficult to see that $\langle F\rangle_{O_{D}}$ is the smallest clone containing $F$.

The set $L_{D}=\left\{F \subseteq \mathrm{O}_{D} \mid F \leq \mathrm{O}_{D}\right\}$ of all clones on $D$ forms a complete lattice $^{1}$ with respect to the set-theoretic inclusion. This lattice will be denoted by $\mathcal{L}_{D}:=\left(L_{D}, \subseteq\right)$. The smallest element of this lattice is $J_{D}$ while the greatest element is $\mathrm{O}_{D}$.

Below, we freely use $\leq$ to denote also the usual order on integers. Nevertheless, we hope not to confuse the reader and be clear.

The $k$-ary constant operation (briefly, constant) $c_{a}^{k}: D^{k} \longrightarrow D$ is given by

$$
c_{a}^{k}\left(x_{1}, \ldots, x_{k}\right):=a
$$

for all $x_{1}, \ldots, x_{k} \in D$, where $a$ is a fixed element of $D$. Moreover, a $k$-ary operation $f$ on $D$ is called monotone, if for all $x_{1}, \ldots, x_{k}, y_{1}, \ldots, y_{k} \in D, x_{1} \leq y_{1}, \ldots, x_{k} \leq y_{k}$ implies

$$
f\left(x_{1}, \ldots, x_{k}\right) \leq f\left(y_{1}, \ldots, y_{k}\right) .
$$

Another example of a clone is the set

$$
M:=\left\{f:\{0,1\}^{k} \longrightarrow\{0,1\} \mid f \text { is monotone }, k \in \mathbb{N}_{+}\right\},
$$

\footnotetext{
${ }^{1}$ In this work some notions as lattice, complete lattice, and ordered set will be not defined. More information about these concepts can be found in [DP02].
} 
and is called the clone of monotone Boolean functions.

Let $m$ be a positive natural number. An $m$-ary relation $\varrho$ on $D$ is a subset of the $m$-fold Cartesian product $D^{m}$. If $\varrho=\left\{r_{1}, \ldots, r_{k}\right\}$ then it is often convenient to represent $\varrho$ as a matrix $\left(r_{i j}\right)_{\substack{1 \leq i \leq m \\ 1 \leq j \leq k}} \in D^{m \times k}$, whose columns are the tuples in the relation, i.e. $r_{j}=\left(r_{1 j}, r_{2 j}, \ldots, r_{m j}\right)$ for all $j \in\{1, \ldots, k\}$. We define by

$$
\mathrm{R}_{D}^{(m)}:=\left\{\varrho \mid \varrho \subseteq D^{m}\right\}
$$

the set of all $m$-ary relations on $D$ and by

$$
\mathrm{R}_{D}:=\bigcup_{m=1}^{\infty} \mathrm{R}_{D}^{(m)}
$$

the set of all finitary relations on $D$.

For $m \in \mathbb{N}_{+}$and an equivalence relation $\theta$ on the set $\{0, \ldots, m-1\}$, we define an $m$-ary relation $d_{\theta}$ on $D$ to be the relation

$$
d_{\theta}:=\left\{\left(x_{0}, \ldots, x_{m-1}\right) \in D^{m} \mid \forall(i, j) \in \theta: x_{i}=x_{j}\right\}
$$

and call it diagonal relation. The special case $d_{\theta}=D^{m}$ is also called trivial relation. The set of all diagonal relations together with the empty relation $\emptyset$ is denoted by $\operatorname{diag}(D)$.

There are only two binary diagonal relations, namely

$$
\begin{aligned}
& \Delta_{D}^{(2)}:=\{(x, x) \mid x \in D\} \quad \text { and } \\
& \nabla_{D}^{(2)}:=\{(x, y) \mid x, y \in D\}=D^{2}
\end{aligned}
$$

the identity relation and the full Cartesian product of $D$ with itself.

\subsection{Relational clones}

The definition to come will serve as an analogy of clones of operations with respect to the Galois connection $\mathrm{Pol}_{D}-\operatorname{Inv}_{D}$. The latter is defined in the next section.

A set $Q$ of finitary relations on $D$ containing all diagonal relations and being closed under composition using primitive positive formulas of first order logic with equality, is called a relational clone on $D$. The last statement is equivalently characterised by the fact [Beh09] that the set of finitary relations is closed under the following (infinite number of) partial operations on $\mathrm{R}_{D}$ : First, the two constants $\emptyset$ and $\Delta_{D}^{(2)}$; then for all arities $m, n \in \mathbb{N}_{+}$and mappings $\alpha:\{1, \ldots, n\} \longrightarrow\{1, \ldots, m\}$ the partial operations $\cap^{(m)}, W_{\alpha}$ and $V_{\alpha}$, where

$$
\begin{aligned}
\cap^{(m)}: \mathrm{R}_{D}^{(m)} \times \mathrm{R}_{D}^{(m)} & \longrightarrow \mathrm{R}_{D}^{(m)} \\
(\varrho, \sigma) & \mapsto \varrho \cap \sigma
\end{aligned}
$$


is the theoretic intersection of relations of the same arity,

$$
\begin{aligned}
W_{\alpha}: \mathrm{R}_{D}^{(n)} & \longrightarrow \mathrm{R}_{D}^{(m)} \\
\varrho & \mapsto\left\{\left(a_{1}, \ldots, a_{m}\right) \in D^{m} \mid\left(a_{\alpha(1)}, \ldots, a_{\alpha(n)}\right) \in \varrho\right\}
\end{aligned}
$$

is the covariant substitution functor ${ }^{2}$ (applied to $\alpha$ ), and

$$
\begin{aligned}
V_{\alpha}: \mathrm{R}_{D}^{(m)} & \longrightarrow \mathrm{R}_{D}^{(n)} \\
\sigma & \mapsto\left\{\left(a_{\alpha(1)}, \ldots, a_{\alpha(n)}\right) \in D^{n} \mid\left(a_{1}, \ldots, a_{m}\right) \in \sigma\right\}
\end{aligned}
$$

is the contravariant substitution functor ${ }^{3}$ (applied to $\alpha$ ).

In [Pös80] has been shown that by a special choice of $\alpha$ we obtain many wellknown operations on $\mathrm{R}_{D}$, below are given some of these operations, which will be used in the subsequent chapters.

1.2.1 Examples. Let $m \in \mathbb{N}_{+}, \varrho \in \mathrm{R}_{D}^{(m)}, \mathbf{m}:=\{1, \ldots, m\}$ and let $\mathrm{S}_{\mathbf{m}}$ be the symmetric group (of all permutations) on $\mathbf{m}$. By a special choice of a mapping $\alpha$ we obtain the following operations on relations:

1) Cyclic permutation of coordinates:

For $\alpha \in \mathrm{S}_{\mathbf{m}}$ defined by $\alpha(i):=(i \bmod m)+1$, we get

$$
W_{\alpha}(\varrho)=\left\{\left(x_{1}, x_{2}, \ldots, x_{m}\right) \in D^{m} \mid\left(x_{2}, x_{3}, \ldots, x_{m}, x_{1}\right) \in \varrho\right\}=: \zeta(\varrho) .
$$

2) Exchange of the first two coordinates:

For $\alpha \in \mathrm{S}_{\mathbf{m}}$ such that $\alpha(i):= \begin{cases}1 & \text { if } i=2, \\ 2 & \text { if } i=1, \\ i & \text { otherwise, }\end{cases}$

we get $W_{\alpha}(\varrho)=\left\{\left(x_{1}, x_{2}, \ldots, x_{m}\right) \in D^{m} \mid\left(x_{2}, x_{1}, x_{3}, \ldots, x_{m}\right) \in \varrho\right\}=: \tau(\varrho)$.

3) Permutation of coordinates:

For $\pi \in \mathrm{S}_{\mathbf{m}}$, we have $W_{\pi^{-1}}=V_{\pi}$.

4) Identification of coordinates:

For $\alpha: \mathbf{m} \longrightarrow\{1, \ldots, m-1\}$ such that $\alpha(i):= \begin{cases}m-1 & \text { if } i=m, \\ i & \text { otherwise }\end{cases}$

we get

$$
W_{\alpha}(\varrho)=\left\{\left(x_{1}, x_{2}, \ldots, x_{m-1}\right) \in D^{m-1} \mid\left(x_{1}, x_{2}, \ldots, x_{m-1}, x_{m-1}\right) \in \varrho\right\}=: \Delta(\varrho) .
$$

\footnotetext{
${ }^{2}$ The name is due to $W_{\alpha_{1}} \circ W_{\alpha_{2}}=W_{\alpha_{1} \circ \alpha_{2}}$
}

${ }^{3}$ Likewise, it holds $V_{\alpha_{1}} \circ V_{\alpha_{2}}=V_{\alpha_{2} \circ \alpha_{1}}$ 
5) Projections onto coordinates:

For injective $\alpha:\{1, \ldots, q\} \longrightarrow \mathbf{m}$ defined by $\alpha(k) \mapsto i_{k}$, we get

$$
\begin{aligned}
V_{\alpha}(\varrho) & =p r_{i_{1}, \ldots, i_{q}}(\varrho) \\
& :=\left\{\left(x_{i_{1}}, \ldots, x_{i_{q}}\right) \mid \exists x_{j}\left(j \in \mathbf{m} \backslash\left\{i_{1}, \ldots, i_{q}\right\}\right):\left(x_{1}, \ldots, x_{m}\right) \in \varrho\right\} .
\end{aligned}
$$

Since the intersection of an arbitrary family of relational clones on $D$ is also a relational clone, we can define the relational clone generated by a subset $Q$ of $\mathrm{R}_{D}$, in analogy to Remark 1.1.2, as the intersection of all relational clones that contain $Q$. This relational clone will be denoted by $[Q]_{\mathrm{R}_{D}}$, and it is the least relational clone containing $Q$. If a subset $Q$ of $\mathrm{R}_{D}$ is a relational clone, it will be denoted by $Q \leq \mathrm{R}_{D}$

1.2.2 Remark ([PK79]). The set $\operatorname{diag}(D)$ of all diagonal relations on $D$ is a relational clone. Furthermore, it is the smallest relational clone, which is contained in every relational clone $Q \leq \mathrm{R}_{D}$. By definition the set $\mathrm{R}_{D}$ is the greatest relational clone.

The complete lattice of relational clones will be denoted by $\mathcal{L}_{D}^{*}:=\left(L_{D}^{*}, \subseteq\right)$, where

$$
L_{D}^{*}=\left\{Q \subseteq \mathrm{R}_{D} \mid Q \leq \mathrm{R}_{D}\right\}
$$

The greatest and smallest element of $\mathcal{L}_{D}^{*}$ are $\mathrm{R}_{D}$ and $\operatorname{diag}(D)$, respectively. For a finite set $D$ the lattice of all relational clones and of all clones are dually isomorphic. Figure 1.1 illustrates this interconnection via the Galois connection $\operatorname{Pol}_{D}-\operatorname{Inv}_{D}$.

In [Pos41], E., L. Post investigated and completely described all elements of the clone lattice $\mathcal{L}_{\{0,1\}}$. Figure 3.1 after Lemma 3.2.10, shows the structure of this lattice, this figure also describes the structure of the lattice of all relational clones on $D$ if we turn it around.

\subsection{The Galois connection Pol - Inv}

Galois connections provide a very useful tool for the description of closure operators. In addition to this, on a finite set clones of operations and relational clones are in one-to-one correspondence via the Galois connection of polymorphisms and invariant relations.

We will start with the definition of a closure operator. As usual for a set $M$, the set of all subsets of $M$ will be denoted by $\mathcal{P}(M)$.

1.3.1 Definition ([DW02]). A mapping $C: \mathcal{P}(M) \longrightarrow \mathcal{P}(M)$ is called a closure operator on $M$, if for all subsets $X, Y \subseteq M$ the following properties are satisfied:

(i) $X \subseteq C(X)$

(extensivity),

(ii) $X \subseteq Y \Rightarrow C(X) \subseteq C(Y)$

(monotonicity),

(iii) $C(X)=C(C(X))$

(idempotency). 
Subsets of $M$ of the form $C(X)$ are called closed (with respect to the operator $C$ ) and $C(X)$ is said to be the closed set generated by $X$.

Next we provide the canonical definition of a Galois connection between sets $M_{1}$ and $M_{2}$.

1.3.2 Definition ([Pös04]). Let $M_{1}$ and $M_{2}$ be two sets and let $\varrho \subseteq M_{1} \times M_{2}$ be a binary relation. The pair $(\varphi, \psi)$ of mappings, defined by

$$
\begin{aligned}
\varphi: \mathcal{P}\left(M_{1}\right) & \longrightarrow \mathcal{P}\left(M_{2}\right) \\
X & \mapsto \varphi(X):=\left\{y \in M_{2} \mid \forall x \in X:(x, y) \in \varrho\right\},
\end{aligned}
$$

and

$$
\begin{aligned}
\psi: \mathcal{P}\left(M_{2}\right) & \longrightarrow \mathcal{P}\left(M_{1}\right) \\
Y & \mapsto \psi(Y):=\left\{x \in M_{1} \mid \forall y \in Y:(x, y) \in \varrho\right\}
\end{aligned}
$$

is called the Galois connection induced by $\varrho$.

It is well-known that such pairs $(\varphi, \psi)$ of mappings can be characterised as follows.

For all $X, X^{\prime} \in \mathcal{P}\left(M_{1}\right)$ and all $Y, Y^{\prime} \in \mathcal{P}\left(M_{2}\right)$ the following conditions are satisfied:

(i) $X \subseteq X^{\prime} \Rightarrow \varphi(X) \supseteq \varphi\left(X^{\prime}\right) \quad$ and $\quad Y \subseteq Y^{\prime} \Rightarrow \psi(Y) \supseteq \psi\left(Y^{\prime}\right)$;

(ii) $X \subseteq \psi \varphi(X) \quad$ and $\quad Y \subseteq \varphi \psi(Y)$.

These conditions imply that $\varphi$ and $\psi$ are anti-monotone and their compositions $\psi \varphi$ and $\varphi \psi$ are closure operators. Therefore, elements $X \in \mathcal{P}\left(M_{1}\right)$ and $Y \in \mathcal{P}\left(M_{2}\right)$ that are closed under $\varphi \psi$ or $\psi \varphi$, respectively, i.e.

$$
X=\psi \varphi(X) \text { and } Y=\varphi \psi(Y) \text {, }
$$

are called Galois closed with respect to the Galois connection $(\varphi, \psi)$. The sets of Galois closed elements are

$$
\left\{\psi(Y) \mid Y \subseteq M_{2}\right\} \subseteq \mathcal{P}\left(M_{1}\right) \text { and }\left\{\varphi(X) \mid X \subseteq M_{1}\right\} \subseteq \mathcal{P}\left(M_{2}\right),
$$

and they form dually isomorphic complete lattices (w.r.t. inclusion).

Next, we will consider Galois connections between sets of operations and relations. They all are based on the preservation relation, which is the most important and basic notion of our investigations.

1.3.3 Definition ([Pös04]). We say that a $k$-ary operation $f$ of $\mathrm{O}_{D}^{(k)}$ preserves an $m$-ary relation $\varrho$ of $\mathrm{R}_{D}^{(m)}$, or $\varrho$ is invariant for $f$, or $f$ is a polymorphism of $\varrho$, denoted by $f \triangleright \varrho$, if whenever

$$
r_{1}=\left(a_{11}, \ldots, a_{m 1}\right) \in \varrho, \ldots, r_{k}=\left(a_{1 k}, \ldots, a_{m k}\right) \in \varrho,
$$

it follows that the following tuple also belongs to $\varrho$ :

$$
f\left[r_{1}, \ldots, r_{k}\right]:=\left(f\left(a_{11}, \ldots, a_{1 k}\right), \ldots, f\left(a_{m 1}, \ldots, a_{m k}\right)\right) \in \varrho .
$$


To illustrate this definition, we represent $\varrho=\left\{r_{1}, \ldots, r_{k}\right\}$ as an $m \times k$-matrix. If we apply $f$ to the rows of the matrix and interpret the $m$ resulting values as a $m$-tuple, this has to be in $\varrho$ as well,

$$
\begin{aligned}
& f\left(\begin{array}{llll}
a_{11} & a_{12} & \ldots & a_{1 k}
\end{array}\right)=0 \\
& f\left(\begin{array}{llll}
a_{21} & a_{22} & \ldots & a_{2 k}
\end{array}\right)=0 \\
& \begin{array}{llll}
\vdots & \vdots & \ddots & \vdots
\end{array} \\
& f\left(\begin{array}{llll}
a_{m 1} & a_{m 2} & \ldots & a_{m k}
\end{array}\right)=0 \\
& m \pi \ldots \pi n \\
& \varrho \quad \varrho \quad \cdots \quad \varrho \quad \Rightarrow \quad \varrho .
\end{aligned}
$$

This preservation relation $\triangleright$ induces a Galois connection between sets of operations and sets of relations on $D$.

1.3.4 Definition ([Pös04]). For a set $F \subseteq \mathrm{O}_{D}$ of operations, we define $\operatorname{Inv}_{D} F$ as the set of all relations that are invariant for all $f \in F$ :

$$
\operatorname{Inv}_{D} F:=\left\{\varrho \in \mathrm{R}_{D} \mid \forall f \in F: f \triangleright \varrho\right\}
$$

Similarly, for a set $Q \subseteq \mathrm{R}_{D}$ of relations, we define

$$
\mathrm{Pol}_{D} Q:=\left\{f \in \mathrm{O}_{D} \mid \forall \varrho \in Q: f \triangleright \varrho\right\}
$$

as the set of polymorphisms of $Q$. Furthermore, for $k \in \mathbb{N}_{+}$we abbreviate

$$
\operatorname{Pol}_{D}^{(k)} Q:=\mathrm{O}_{D}^{(k)} \cap \operatorname{Pol}_{D} Q
$$

If $D$ is known from the context we write Pol instead of $\mathrm{Pol}_{D}$, and Inv instead of $\operatorname{Inv}_{D}$. The operators Pol and Inv define the Galois connection Pol-Inv, see Theorem 1.3.5 below.

We can restrict this Galois connection on the operational side, by setting

$$
\text { End } Q:=\mathrm{O}_{D}^{(1)} \cap \operatorname{Pol} Q \quad \text { and } \quad \text { Aut } Q:=\mathrm{S}_{D} \cap \operatorname{Pol} Q
$$

and call these sets of operations endomorphisms of $Q$ and automorphisms ${ }^{4}$ of $Q$, respectively. In this context $\mathrm{S}_{D}$ denotes the symmetric group on $D$.

\footnotetext{
${ }^{4}$ In general such relation preserving permutations are called weak automorphisms of $Q$. However, Lemma 4.1.4 below states that for finite carrier sets relation preserving permutations are also relation reflecting, i.e. $f, f^{-1} \in \operatorname{Pol} Q$. Hence in our case the notions of weak automorphism and automorphism of $Q$ coincide.
} 


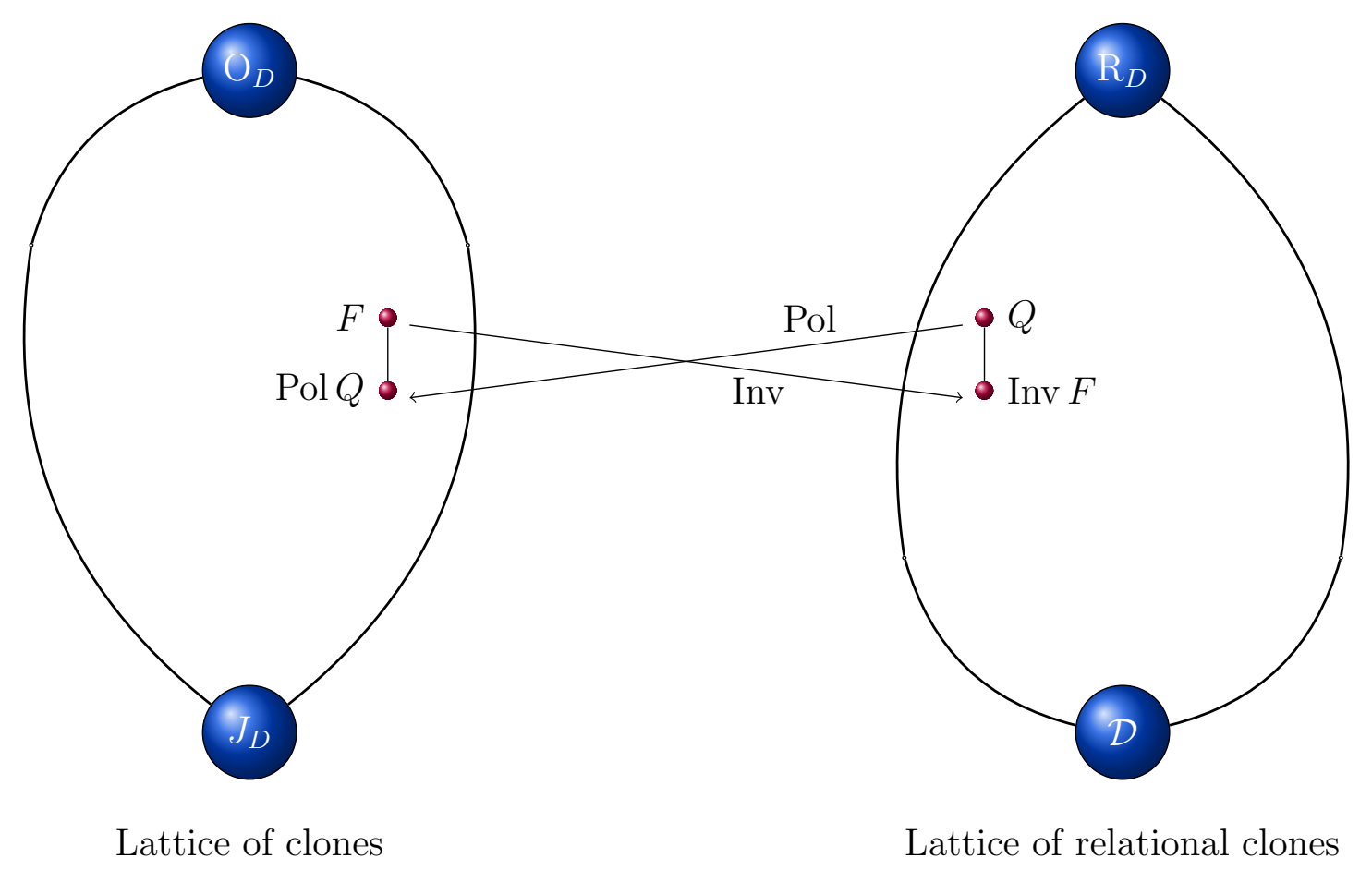

Figure 1.1: Interconnection between clones and relational clones. Here, $\mathcal{D}$ denotes the relational clone $\operatorname{diag}(D)$.

The following theorem gives essential properties of the operators Pol and Inv. They will be used many times in the subsequent chapters.

1.3.5 Theorem ([PK79]). The operators Pol and Inv define a Galois connection between sets of operations and sets of relations on $D$, i.e., it follows that

$$
\begin{array}{ll}
Q \subseteq \operatorname{Inv} \operatorname{Pol} Q, & Q_{1} \subseteq Q_{2} \Rightarrow \operatorname{Pol} Q_{1} \supseteqq \operatorname{Pol} Q_{2}, \\
F \subseteq \operatorname{Pol} \operatorname{Inv} F, & F_{1} \subseteq F_{2} \Rightarrow \operatorname{Inv} F_{1} \supseteqq \operatorname{Inv} F_{2},
\end{array}
$$

Pol Inv $\operatorname{Pol} Q=\operatorname{Pol} Q, \quad \operatorname{Inv} \operatorname{Pol} \operatorname{Inv} F=\operatorname{Inv} F$

for $Q, Q_{1}, Q_{2} \subseteq \mathrm{R}_{D}$ and $F, F_{1}, F_{2} \subseteq \mathrm{O}_{D}$

Furthermore, it follows that

$$
\begin{array}{cc}
Q \subseteq \operatorname{Inv} F \Longleftrightarrow F \subseteq \operatorname{Pol} Q, & \\
\operatorname{Pol} Q_{1} \cap \operatorname{Pol} Q_{2}=\operatorname{Pol}\left(Q_{1} \cup Q_{2}\right), & \operatorname{Pol} Q=\bigcap_{\varrho \in Q} \operatorname{Pol} \varrho, \\
\operatorname{Inv} F_{1} \cap \operatorname{Inv} F_{2}=\operatorname{Inv}\left(F_{1} \cup F_{2}\right), & \operatorname{Inv} F=\bigcap_{f \in F} \operatorname{Inv} f .
\end{array}
$$

The lemma to come shows that for a set of relations $Q$, the set $\operatorname{Pol} Q$ is always a clone. 
1.3.6 Lemma ([PK79]). Let $m \in \mathbb{N}_{+}$, and let $Q \subseteq \mathrm{R}_{D}^{(m)}$ be a set of $m$-ary relations on $D$. Then the set $\operatorname{Pol} Q$ is a clone.

Proof. First, we prove $J_{D} \subseteq \operatorname{Pol} Q$. We show that $e_{j}^{k} \in \operatorname{Pol} Q$ for each $j \in\{1, \ldots, k\}$, where $k \in \mathbb{N}_{+}$. Consider any tuples $r_{1}, \ldots, r_{k}$ belonging to $\varrho \in Q$. For each $j \in\{1, \ldots, k\}$ we have that

$$
e_{j}^{k}\left[r_{1}, \ldots, r_{k}\right]=r_{j} \in \varrho
$$

Thus, $e_{j}^{k} \triangleright \varrho$.

Let $k, m \in \mathbb{N}_{+}, f \in \mathrm{Pol}^{(k)} Q$, and $g_{1}, \ldots, g_{k} \in \mathrm{Pol}^{(m)} Q$, we have to prove that $f\left(g_{1}, \ldots, g_{k}\right) \in \mathrm{Pol}^{(m)} Q$. Consider any tuples $r_{1}, \ldots, r_{m}$ belonging to $\varrho \in Q$. Then

$$
\left(f\left(g_{1}, \ldots, g_{k}\right)\right)\left[r_{1}, \ldots, r_{m}\right]=f\left(g_{1}\left[r_{1}, \ldots, r_{m}\right], \ldots, g_{k}\left[r_{1}, \ldots, r_{m}\right]\right)
$$

Because $g_{1} \triangleright \varrho, \ldots, g_{k} \triangleright \varrho$, we obtain $g_{1}\left[r_{1}, \ldots, r_{m}\right] \in \varrho, \ldots, g_{k}\left[r_{1}, \ldots, r_{m}\right] \in \varrho$. Since $f \triangleright \varrho$, we get $f\left(g_{1}\left[r_{1}, \ldots, r_{m}\right], \ldots, g_{k}\left[r_{1}, \ldots, r_{m}\right]\right) \in \varrho$. Hence, $f\left(g_{1}, \ldots, g_{k}\right) \triangleright \varrho$.

Let us finish this section explaining the relationship between clone generation and the Galois connection Pol - Inv.

As for $F \subseteq \mathrm{O}_{D}$ and $Q \subseteq \mathrm{R}_{D}$, Pol $Q$ is a clone and Inv $F$ a relational clone, one obtains

$$
\begin{aligned}
\langle F\rangle_{O_{D}} & \subseteq \operatorname{Pol} \operatorname{Inv} F \\
{[Q]_{\mathrm{R}_{D}} } & \subseteq \operatorname{Inv} \operatorname{Pol} Q
\end{aligned}
$$

For a finite set $D$ it is well-known (cf. [PK79]) that the Galois closed sets with respect to the Galois connection Pol - Inv are precisely the clones and relational clones, i.e.

1.3.7 Theorem ([PK79]). For $F \subseteq \mathrm{O}_{D}$ and $Q \subseteq \mathrm{R}_{D}$.

$$
\begin{aligned}
& \langle F\rangle_{O_{D}}=\operatorname{Pol} \operatorname{Inv} F \\
& {[Q]_{\mathrm{R}_{D}}=\operatorname{Inv} \operatorname{Pol} Q}
\end{aligned}
$$

The Galois closed sets of relations of the Galois connection Aut - Inv are special relational clones on $D$, so-called Krasner clones. They are exactly those relational clones that are additionally closed under finite unions of relations of the same arity and under complements of relations. Moreover, the Galois closed sets of relations of the Galois connection End - Inv are relational clones on D, so-called weak-Krasner clones. They are those relational clones that are additionally closed under finite unions of relations of the same arity. 


\subsection{The Constraint Satisfaction Problem}

In the current dissertation we will investigate if the Constraint Satisfaction Problem over a special set of relations, which is defined in the next chapter, is tractable. Hence, some basic concepts and results associated to Constraint Satisfaction Problems are addressed in this section.

The aim in a Constraint Satisfaction Problem is to find an assignment of values to a given set of variables, subject to constraints on the values which can be assigned simultaneously to certain specified subsets of the variables [BKJ00]. Many combinatorial problems appearing in computer science and artificial intelligence can be expressed as particular subclasses of the CSP(cf. [Bul02]).

The general CSP is NP-complete, but certain restrictions on the form of the involved constraints may allow to solve the problem in polynomial time.

1.4.1 Definition ([BKJ00]). The constraint satisfaction problem (CSP) over an arbitrary subset $Q$ of $\mathrm{R}_{D}$, denoted $\operatorname{CSP}(Q)$, is defined to be the decision problem with instance $(V, D, \mathcal{C})$, where

- Instance: $V$ is a set of finite variables; $D$ is a set of finite values (sometimes called a domain); and $\mathcal{C}$ is a set of constraints, $\left\{C_{1}, \ldots, C_{q}\right\}$.

Each constraint $C_{i} \in \mathcal{C}$ is a pair $\left\langle s_{i}, \varrho_{i}\right\rangle$, where $s_{i}$ is a tuple of variables of length $m_{i}$, called the constraint scope, and $\varrho_{i} \in Q$ an $m_{i}$-ary relation on $D$, called the constraint relation.

- Question: Does there exists a solution to $(V, D, \mathcal{C})$, that is, a function $f$ from $V$ to $D$, such that for each constraint $\left\langle s_{i}, \varrho_{i}\right\rangle \in \mathcal{C}$, with $s_{i}=\left(x_{i_{1}}, \ldots, x_{i_{m}}\right)$, the tuple $\left(f\left(x_{i_{1}}\right), \ldots, f\left(x_{i_{m}}\right)\right)$ belongs to $\varrho_{i}$ ?

1.4.2 Definition ([JCG97]). If there exists an algorithm that solves every problem instance in $\operatorname{CSP}(Q)$ in polynomial time, then we shall say that $\operatorname{CSP}(Q)$ is a tractable problem, and $Q$ is a tractable set of relations.

P. Jeavons has proved a strong theorem connecting the complexity of $\operatorname{CSP}(Q)$ to the clone of operations $\operatorname{Pol} Q$.

1.4.3 Theorem ([Jea98]). Let $D$ be a finite set, and $Q, Q_{0} \subseteq \mathrm{R}_{D}$. If $Q_{0}$ is finite and $\operatorname{Pol} Q \subseteq \operatorname{Pol} Q_{0}$, then $\operatorname{CSP}\left(Q_{0}\right)$ is reducible in polynomial time to $\operatorname{CSP}(Q)$.

Informally speaking, Theorem 1.4 .3 says that the complexity of $\operatorname{CSP}(Q)$ is determined by the clone $\operatorname{Pol} Q$.

Let us finish this chapter by giving the following sufficient condition to ensure tractability.

1.4.4 Proposition ([JCG97]). Let $c_{a}$ be any constant operation with value a on $D$. For any subset $Q$ of $\mathrm{R}_{D}$, if $c_{a} \in \operatorname{Pol} Q$ then $\operatorname{CSP}(Q)$ is solvable in polynomial time. 


\section{Clausal relations}

In this chapter we study a special set of relations so-called clausal relations. The name of this type of relations is justified by the fact that clausal relations on a 2-element set are exactly those representable by clauses, that is, disjunctions of variables and their negations. In Section 2.2 we discuss some operations under which clausal relations are closed.

\subsection{Introduction}

Clones that are determined by sets of clausal relations will be thoroughly discussed in the following chapters. Next, these relations will be defined.

2.1.1 Definition. Let $p, q \in \mathbb{N}_{+}$. For given parameters $\mathbf{a}=\left(a_{1}, \ldots, a_{p}\right) \in D^{p}$ and $\mathbf{b}=\left(b_{1}, \ldots, b_{q}\right) \in D^{q}$, the clausal relation $\mathbf{R}_{\mathbf{b}}^{\mathbf{a}}$ of type $(p, q)$ is the set of all tuples $\left(x_{1}, \ldots, x_{p}, y_{1}, \ldots, y_{q}\right) \in D^{p+q}$ satisfying

$$
\left(x_{1} \geq a_{1}\right) \vee \ldots \vee\left(x_{p} \geq a_{p}\right) \vee\left(y_{1} \leq b_{1}\right) \vee \ldots \vee\left(y_{q} \leq b_{q}\right)
$$

Note that whenever $a_{i}=0$ for some $i \in\{1, \ldots, p\}$ or $b_{j}=n-1$ for some $j \in\{1, \ldots, q\}$, then the relation $\mathrm{R}_{\mathbf{b}}^{\mathbf{a}}$ is the full Cartesian power of $D$, i.e. $\mathbf{R}_{\mathbf{b}}^{\mathbf{a}}=D^{p+q}$, because $(2.1)$ is satisfied for any $\left(x_{1}, \ldots, x_{p}, y_{1}, \ldots, y_{q}\right) \in D^{p+q}$.

2.1.2 Definition. Let $p, q \in \mathbb{N}_{+}$. We use

$$
\mathcal{R}_{q}^{p}:=\left\{\mathrm{R}_{\mathbf{b}}^{\mathbf{a}} \mid \mathbf{a} \in D^{p}, \mathbf{b} \in D^{q}\right\}
$$

to denote the set of all clausal relations of arity ${ }^{1} p+q$ and

$$
C \mathrm{R}_{D}:=\bigcup_{(p, q) \in \mathbb{N}_{+}^{2}} \mathcal{R}_{q}^{p}
$$

for the set of all finitary clausal relations on $D$.

We write $\mathrm{R}_{\mathbf{b}}^{a}$ for $\mathrm{R}_{\mathbf{b}}^{(a)}$ in the case $p=1$ and likewise $\mathrm{R}_{b}^{\mathrm{a}}$ for $\mathrm{R}_{(b)}^{\mathrm{a}}$ in the case $q=1$. Furthermore, we avoid the parentheses of the expression (2.1).

Bellow we give two examples of clausal relations.

\footnotetext{
${ }^{1}$ If we speak of a clausal relation of arity $p+q$, we implicitly mean also that the clausal relation is of type $(p, q)$.
} 


\subsubsection{Examples.}

a) Let $D=\{0,1\}$, then

$$
\mathrm{R}_{1}^{0}=\left\{\left(x_{1}, y_{1}\right) \in D^{2} \mid x_{1} \geq 0 \vee y_{1} \leq 1\right\}=\left(\begin{array}{cccc}
0 & 0 & 1 & 1 \\
0 & 1 & 0 & 1
\end{array}\right)=D^{2}
$$

b) Let $D=\{0,1,2\}$, then

$$
\begin{aligned}
\mathrm{R}_{0}^{(2,2)} & =\left\{\left(x_{1}, x_{2}, y_{1}\right) \in D^{3} \mid x_{1} \geq 2 \vee x_{2} \geq 2 \vee y_{1} \leq 0\right\} \\
& =\left(\begin{array}{lllllllllllllllllllllllllll}
0 & 0 & 0 & 0 & 0 & 1 & 1 & 1 & 1 & 1 & 2 & 2 & 2 & 2 & 2 & 2 & 2 & 2 & 2 \\
0 & 1 & 2 & 2 & 2 & 0 & 1 & 2 & 2 & 2 & 0 & 0 & 0 & 1 & 1 & 1 & 2 & 2 & 2 \\
0 & 0 & 0 & 1 & 2 & 0 & 0 & 0 & 1 & 2 & 0 & 1 & 2 & 0 & 1 & 2 & 0 & 1 & 2
\end{array}\right) .
\end{aligned}
$$

\subsection{Operations on clausal relations}

In this section we investigate under which operations (namely $\bigcap, \cup, W_{\alpha}, V_{\alpha}$ ) clausal relations are closed. Then, we prove that a union of two clausal relations is also a clausal relation.

2.2.1 Lemma. Let $p, q \in \mathbb{N}_{+}$. If $\mathrm{R}_{\mathbf{b}}^{\mathbf{a}}$ and $\mathrm{R}_{\mathbf{b}^{\prime}}^{\mathbf{a}^{\prime}}$ are clausal relations of arity $p+q$, where the parameters $\mathbf{a}=\left(a_{1}, \ldots, a_{p}\right), \mathbf{a}^{\prime}=\left(a_{1}^{\prime}, \ldots, a_{p}^{\prime}\right) \in D^{p}$ and $\mathbf{b}=\left(b_{1}, \ldots, b_{q}\right)$, $\mathbf{b}^{\prime}=\left(b_{1}^{\prime}, \ldots, b_{q}^{\prime}\right) \in D^{q}$. Then it follows that

$$
\mathrm{R}_{\mathrm{b}}^{\mathrm{a}} \cup \mathrm{R}_{\mathrm{b}^{\prime}}^{\mathbf{a}^{\prime}}=\mathrm{R}_{\mathrm{d}}^{\mathbf{c}}
$$

where $\mathbf{c}=\left(\min \left\{a_{1}, a_{1}^{\prime}\right\}, \ldots, \min \left\{a_{p}, a_{p}^{\prime}\right\}\right)$ and $\mathbf{d}=\left(\max \left\{b_{1}, b_{1}^{\prime}\right\}, \ldots, \max \left\{b_{q}, b_{q}^{\prime}\right\}\right)$.

Proof. Let $\mathbf{z}=\left(x_{1}, \ldots, x_{p}, y_{1}, \ldots, y_{q}\right) \in \mathrm{R}_{\mathbf{b}}^{\mathbf{a}} \cup \mathrm{R}_{\mathbf{b}^{\prime}}^{\mathbf{a}^{\prime}}$

$$
\begin{aligned}
& \Longleftrightarrow \quad x_{1} \geq a_{1} \vee \ldots \vee x_{p} \geq a_{p} \vee y_{1} \leq b_{1} \vee \ldots \vee y_{q} \leq b_{q} \vee \\
& x_{1} \geq a_{1}^{\prime} \vee \ldots \vee x_{p} \geq a_{p}^{\prime} \vee y_{1} \leq b_{1}^{\prime} \vee \ldots \vee y_{q} \leq b_{q}^{\prime} \\
& \Longleftrightarrow \bigvee_{1 \leq i \leq p}\left(x_{i} \geq a_{i} \vee x_{i} \geq a_{i}^{\prime}\right) \vee \bigvee_{1 \leq j \leq q}\left(y_{j} \leq b_{j} \vee y_{j} \leq b_{j}^{\prime}\right) \\
& \Longleftrightarrow \bigvee_{1 \leq i \leq p}\left(x_{i} \geq \min \left\{a_{i}, a_{i}^{\prime}\right\}\right) \vee \bigvee_{1 \leq j \leq q}\left(y_{j} \leq \max \left\{b_{j}, b_{j}^{\prime}\right\}\right)
\end{aligned}
$$

This is equivalent to $\mathbf{z} \in \mathrm{R}_{\mathbf{d}}^{\mathbf{c}}$.

2.2.2 Lemma. Let $p, q \in \mathbb{N}_{+}$. If $\mathrm{R}_{\mathbf{b}}^{\mathbf{a}}$ and $\mathrm{R}_{\mathbf{b}^{\prime}}^{\mathbf{a}^{\prime}}$ are clausal relations of arity $p+q$, where the parameters $\mathbf{a}=\left(a_{1}, \ldots, a_{p}\right), \mathbf{a}^{\prime}=\left(a_{1}^{\prime}, \ldots, a_{p}^{\prime}\right) \in D^{p}$ and $\mathbf{b}=\left(b_{1}, \ldots, b_{q}\right)$, $\mathbf{b}^{\prime}=\left(b_{1}^{\prime}, \ldots, b_{q}^{\prime}\right) \in D^{q}$. Then it follows that

$$
\mathrm{R}_{\mathbf{b}}^{\mathbf{a}} \cap \mathrm{R}_{\mathbf{b}^{\prime}}^{\mathbf{a}^{\prime}} \supseteq \mathrm{R}_{\mathbf{d}}^{\mathbf{c}}
$$

where $\mathbf{c}=\left(\max \left\{a_{1}, a_{1}^{\prime}\right\}, \ldots, \max \left\{a_{p}, a_{p}^{\prime}\right\}\right)$ and $\mathbf{d}=\left(\min \left\{b_{1}, b_{1}^{\prime}\right\}, \ldots, \min \left\{b_{q}, b_{q}^{\prime}\right\}\right)$. 
Proof. Let $\mathbf{z}=\left(x_{1}, \ldots, x_{p}, y_{1}, \ldots, y_{q}\right) \in \mathrm{R}_{\mathbf{b}}^{\mathbf{a}} \cap \mathrm{R}_{\mathbf{b}^{\prime}}^{\mathbf{a}^{\prime}}$

$$
\begin{aligned}
& \Longleftrightarrow \quad\left[x_{1} \geq a_{1} \vee \ldots \vee x_{p} \geq a_{p} \vee y_{1} \leq b_{1} \vee \ldots \vee y_{q} \leq b_{q}\right] \bigwedge \\
& {\left[x_{1} \geq a_{1}^{\prime} \vee \ldots \vee x_{p} \geq a_{p}^{\prime} \vee y_{1} \leq b_{1}^{\prime} \vee \ldots \vee y_{q} \leq b_{q}^{\prime}\right] } \\
& \Longleftrightarrow {\left[\left(x_{1} \geq a_{1} \vee \ldots \vee x_{p} \geq a_{p}\right) \wedge\left(x_{1} \geq a_{1}^{\prime} \vee \ldots \vee x_{p} \geq a_{p}^{\prime}\right)\right] \vee } \\
& {\left[\left(y_{1} \leq b_{1} \vee \ldots \vee y_{q} \leq b_{q}\right) \wedge\left(x_{1} \geq a_{1}^{\prime} \vee \ldots \vee x_{p} \geq a_{p}^{\prime}\right)\right] \vee } \\
& {\left[\left(x_{1} \geq a_{1} \vee \ldots \vee x_{p} \geq a_{p}\right) \wedge\left(y_{1} \leq b_{1}^{\prime} \vee \ldots \vee y_{q} \leq b_{q}^{\prime}\right)\right] \vee } \\
& {\left[\left(y_{1} \leq b_{1} \vee \ldots \vee y_{q} \leq b_{q}\right) \wedge\left(y_{1} \leq b_{1}^{\prime} \vee \ldots \vee y_{q} \leq b_{q}^{\prime}\right)\right] } \\
& \Longleftrightarrow \bigvee_{1 \leq i \leq p}\left(x_{i} \geq a_{i} \wedge x_{i} \geq a_{i}^{\prime}\right) \vee \bigvee_{1 \leq j \leq q}\left(y_{j} \leq b_{j} \wedge y_{j} \leq b_{j}^{\prime}\right) \\
& \bigvee_{1 \leq i \leq p}\left(x_{i} \geq \max \left\{a_{i}, a_{i}^{\prime}\right\}\right) \vee \bigvee_{1 \leq j \leq q}\left(y_{j} \leq \min \left\{b_{j}, b_{j}^{\prime}\right\}\right) .
\end{aligned}
$$

Thus, $\mathrm{R}_{\mathbf{b}}^{\mathbf{a}} \cap \mathrm{R}_{\mathbf{b}^{\prime}}^{\mathbf{a}^{\prime}} \supseteq \mathrm{R}_{\mathbf{d}}^{\mathbf{c}}$.

In general $\mathrm{R}_{\mathbf{b}}^{\mathbf{a}} \cap \mathrm{R}_{\mathbf{b}^{\prime}}^{\mathbf{a}^{\prime}}=\mathrm{R}_{\mathbf{d}}^{\mathbf{c}}$ does not hold, as is shown in the following example.

2.2.3 Example. Let $D=\{0,1\}$, consider $\mathbf{a}=(0,1), \mathbf{a}^{\prime}=(1,0), b_{1}=0$ and $b_{1}^{\prime}=1$. Let also $\mathbf{z}=\left(x_{1}, x_{2}, y_{1}\right)=(0,0,1)$. The tuple $\mathbf{z}$ belongs to $\mathrm{R}_{b_{1}}^{\mathbf{a}} \cap \mathrm{R}_{b_{1}^{\prime}}^{\mathbf{a}^{\prime}}$, because $\mathrm{R}_{b_{1}}^{\mathrm{a}}=D^{3}=\mathrm{R}_{b_{1}^{\prime}}^{\mathbf{a}^{\prime}}$, but $\mathbf{z} \notin \mathrm{R}_{0}^{(1,1)}$.

A special case of the covariant and the contravariant substitution functor is the permutation of the entries of the tuples in a relation. For clausal relations $R_{b}^{a}$ this works as follows.

Let $p, q \in \mathbb{N}_{+}, \pi_{1} \in \mathrm{S}_{p}, \pi_{2} \in \mathrm{S}_{q}$ and let $\pi_{3}:\{1, \ldots, p+q\} \longrightarrow\{1, \ldots, p+q\}$ such that,

$$
\pi_{3}(i):= \begin{cases}\pi_{1}(i) & \text { if } i \leq p \\ \pi_{2}(i-p) & \text { otherwise }\end{cases}
$$

$$
\begin{aligned}
& W_{\pi_{2}}^{\pi_{1}}\left(\mathrm{R}_{\mathbf{b}}^{\mathbf{a}}\right):=W_{\pi_{3}}\left(\mathrm{R}_{\mathbf{b}}^{\mathbf{a}}\right)=\left\{\left(x_{1}, \ldots, x_{p}, y_{1}, \ldots, y_{q}\right) \in D^{p+q} \mid\right. \\
&\left.\left(x_{\pi_{1}(1)}, \ldots, x_{\pi_{1}(p)}, y_{\pi_{2}(1)}, \ldots, y_{\pi_{2}(q)}\right) \in \mathrm{R}_{\mathbf{b}}^{\mathbf{a}}\right\} . \\
& V_{\pi_{2}}^{\pi_{1}}\left(\mathrm{R}_{\mathbf{b}}^{\mathbf{a}}\right):=V_{\pi_{3}}\left(\mathrm{R}_{\mathbf{b}}^{\mathbf{a}}\right)=\left\{\left(x_{\pi_{1}(1)}, \ldots, x_{\pi_{1}(p)}, y_{\pi_{2}(1)}, \ldots, y_{\pi_{2}(q)}\right) \in D^{p+q} \mid\right. \\
&\left.\left(x_{1}, \ldots, x_{p}, y_{1}, \ldots, y_{q}\right) \in \mathrm{R}_{\mathbf{b}}^{\mathbf{a}}\right\} .
\end{aligned}
$$

2.2.4 Lemma. Let $p, q \in \mathbb{N}_{+}, \pi_{1} \in \mathrm{S}_{p}$ and $\pi_{2} \in \mathrm{S}_{q}$. If $\mathrm{R}_{\mathbf{b}}^{\mathbf{a}}$ is a clausal relation of arity $p+q$, then

$$
\mathrm{R}_{\mathrm{b} \circ \pi_{2}}^{\mathbf{a} \circ \pi_{1}}=V_{\pi_{2}}^{\pi_{1}}\left(\mathrm{R}_{\mathbf{b}}^{\mathbf{a}}\right)
$$

holds, where $\mathbf{a} \circ \pi_{1}=\left(a_{\pi_{1}(1)}, \ldots, a_{\pi_{1}(p)}\right), \mathbf{b} \circ \pi_{2}=\left(b_{\pi_{2}(1)}, \ldots, b_{\pi_{2}(q)}\right)$. 
Proof. Let $(\mathbf{x}, \mathbf{y})=\left(x_{1}, \ldots, x_{p}, y_{1}, \ldots, y_{q}\right) \in D^{p+q}$. Then it follows that

$$
\begin{aligned}
& (\mathbf{x}, \mathbf{y}) \in \mathrm{R}_{\mathbf{b} \circ \pi_{2}}^{\mathbf{a} \circ \pi_{1}} \Leftrightarrow\left(x_{1} \geq a_{\pi_{1}(1)} \vee \ldots \vee x_{p} \geq a_{\pi_{1}(p)} \vee y_{1} \leq b_{\pi_{2}(1)} \vee \ldots \vee y_{q} \leq b_{\pi_{2}(q)}\right) \\
& \Leftrightarrow\left(x_{\pi_{1}^{-1}(1)} \geq a_{1} \vee \ldots \vee x_{\pi_{1}^{-1}(p)} \geq a_{p} \vee y_{\pi_{2}^{-1}(1)} \leq b_{1} \vee \ldots \vee y_{\pi_{2}^{-1}(q)} \leq b_{q}\right) \\
& \Leftrightarrow\left(x_{\pi_{1}^{-1}(1)}, \ldots, x_{\pi_{1}^{-1}(p)}, y_{\pi_{2}^{-1}(1)}, \ldots, y_{\pi_{2}^{-1}(q)}\right) \in \mathrm{R}_{\mathbf{b}}^{\mathbf{a}} \\
& \Leftrightarrow(\mathbf{x}, \mathbf{y}) \in W_{\pi_{2}^{-1}}^{\pi_{1}^{-1}}\left(\mathrm{R}_{\mathbf{b}}^{\mathbf{a}}\right) \stackrel{1.2 .1}{\Leftrightarrow}(\mathbf{x}, \mathbf{y}) \in V_{\pi_{2}}^{\pi_{1}}\left(\mathrm{R}_{\mathbf{b}}^{\mathbf{a}}\right) .
\end{aligned}
$$

Subsets of clausal relations are not relational clones, because they are not closed under intersections. Below, we show how the identification of the first two coordinates works in clausal relations $\mathrm{R}_{\mathrm{b}}^{\mathrm{a}}$.

Let $p, q$ be positive natural numbers, and let $\mathrm{R}_{\mathbf{b}}^{\mathbf{a}}$ be a clausal relation of arity $p+q$, we denote by

$$
\begin{aligned}
\Delta^{p}\left(\mathrm{R}_{\mathbf{b}}^{\mathbf{a}}\right):=\left\{\left(x_{1}, \ldots, x_{p-1}, y_{1}, \ldots, y_{q}\right) \in\right. & D^{p-1+q} \mid \\
& \left.\left(x_{1}, x_{1}, x_{2}, \ldots, x_{p-1}, y_{1}, \ldots, y_{q}\right) \in \mathrm{R}_{\mathbf{b}}^{\mathbf{a}}\right\}, \\
\Delta_{q}\left(\mathrm{R}_{\mathbf{b}}^{\mathbf{a}}\right):=\left\{\left(x_{1}, \ldots, x_{p}, y_{1}, \ldots, y_{q-1}\right) \in\right. & D^{p+q-1} \mid \\
& \left.\left(x_{1}, \ldots, x_{p}, y_{1}, y_{1}, y_{2}, \ldots, y_{q-1}\right) \in \mathrm{R}_{\mathbf{b}}^{\mathbf{a}}\right\} .
\end{aligned}
$$

2.2.5 Lemma. Let $p, q \in \mathbb{N}_{+}$and let $\mathrm{R}_{\mathbf{b}}^{\mathbf{a}}$ be a clausal relation of arity $p+q$. Then it follows that

$$
\Delta^{p}\left(\mathrm{R}_{\mathbf{b}}^{\mathbf{a}}\right)=\mathrm{R}_{\mathbf{b}}^{\left(\min \left\{a_{1}, a_{2}\right\}, a_{3} \ldots, a_{p}\right)} .
$$

Proof. Let $\mathbf{z}:=\left(x_{1}, x_{2}, \ldots, x_{p-1}, y_{1}, \ldots, y_{q}\right) \in D^{p-1+q}$. Then it follows that

$$
\begin{aligned}
\mathbf{z} \in \Delta^{p}\left(\mathrm{R}_{\mathbf{b}}^{\mathbf{a}}\right) & \Leftrightarrow\left(x_{1}, x_{1}, x_{2}, \ldots, x_{p-1}, y_{1}, \ldots, y_{q}\right) \in \mathrm{R}_{\mathbf{b}}^{\mathbf{a}} \\
& \Leftrightarrow\left(x_{1} \geq a_{1} \vee x_{1} \geq a_{2} \vee x_{2} \geq a_{3} \vee \ldots \vee x_{p-1} \geq a_{p}\right) \vee \bigvee_{1 \leq j \leq q}\left(y_{j} \leq b_{j}\right) \\
& \Leftrightarrow\left(x_{1} \geq \min \left\{a_{1}, a_{2}\right\} \vee x_{2} \geq a_{3} \vee \ldots \vee x_{p-1} \geq a_{p}\right) \vee \bigvee_{1 \leq j \leq q}\left(y_{j} \leq b_{j}\right) \\
& \Leftrightarrow \mathbf{z} \in \mathrm{R}_{\mathbf{b}}^{\left(\min \left\{a_{1}, a_{2}\right\}, a_{3} \ldots, a_{p}\right)}
\end{aligned}
$$

Similarly, one can prove the following lemma.

2.2.6 Lemma. Let $p, q \in \mathbb{N}_{+}$and let $\mathrm{R}_{\mathbf{b}}^{\mathbf{a}}$ be a clausal relation of arity $p+q$. Then it follows that

$$
\Delta_{q}\left(\mathrm{R}_{\mathbf{b}}^{\mathbf{a}}\right)=\mathrm{R}_{\left(\max \left\{b_{1}, b_{2}\right\}, b_{3} \ldots, b_{q}\right)}^{\mathbf{a}} .
$$

From 2.2.5 and 2.2.6, we deduce the corollary below.

2.2.7 Corollary. Let $p, q \in \mathbb{N}_{+}$and let $\mathrm{R}_{\mathbf{b}}^{\mathbf{a}}$ be a clausal relation of arity $p+q$. Then it follows that

$$
\Delta_{q}\left[\Delta^{p}\left(\mathrm{R}_{\mathbf{b}}^{\mathbf{a}}\right)\right]=\mathrm{R}_{\left(\max \left\{b_{1}, b_{2}\right\}, b_{3} \ldots, b_{q}\right)}^{\left(\min \left\{a_{1}, a_{2}\right\}, a_{p}\right)} .
$$


The following handy lemma shows that the only trivial clausal relations are those we already noticed after Definition 2.1.1. Moreover, the non-trivial ones can be easily identified by their parameters $\mathbf{a}$ and $\mathbf{b}$.

2.2.8 Lemma. The set $C \mathrm{R}_{D}$ can be partitioned as

$$
C \mathrm{R}_{D}=\left\{D^{p+q} \mid p, q \in \mathbb{N}_{+}\right\} \dot{\cup} C \mathrm{R}_{D}^{*},
$$

where

$$
\left\{D^{p+q} \mid p, q \in \mathbb{N}_{+}\right\}=C \mathrm{R}_{D} \cap \operatorname{diag}(D)
$$

are the trivial clausal relations and

$$
C \mathrm{R}_{D}^{*}:=\left\{\mathrm{R}_{\mathbf{b}}^{\mathbf{a}} \mid \mathbf{a} \in(D \backslash\{0\})^{p}, \mathbf{b} \in(D \backslash\{n-1\})^{q} ; p, q \in \mathbb{N}_{+}\right\}
$$

are the non-trivial clausal relations.

Proof. Let $p, q \in \mathbb{N}_{+}, \mathbf{a}=\left(a_{1}, \ldots, a_{p}\right) \in D^{p}, \mathbf{b}=\left(b_{1}, \ldots, b_{q}\right) \in D^{q}$. We have observed above that if one of the $a_{1}, \ldots, a_{p}$ equals 0 , or one of the $b_{1}, \ldots, b_{q}$ equals $n-1$, then $\mathrm{R}_{\mathbf{b}}^{\mathbf{a}}$ is a total relation, i.e. $\mathrm{R}_{\mathbf{b}}^{\mathbf{a}}=D^{p+q}$.

We have to show $C \mathrm{R}_{D}^{*} \cap \operatorname{diag}(D)=\emptyset$. Let us assume the existence of a relation $\varrho \in C \mathrm{R}_{D}^{*} \cap \operatorname{diag}(D)$. Then there exist $\mathbf{a} \in(D \backslash\{0\})^{p}$ and $\mathbf{b} \in(D \backslash\{n-1\})^{q}$ such that

$$
\varrho=\mathrm{R}_{\mathbf{b}}^{\mathbf{a}}
$$

and there exists an equivalence relation $\theta$ on the set $\mathbf{m}=\{0, \ldots, m-1\}$ (where $m=p+q)$ such that $\varrho=d_{\theta}$. We show

$$
\theta=\{(x, x) \mid x \in \mathbf{m}\}=\Delta_{\mathbf{m}},
$$

thus

$$
\varrho=d_{\theta}=D^{m} .
$$

This is a contradiction to $\mathrm{R}_{\mathbf{b}}^{\mathbf{a}}=\varrho=D^{m}$, because $(\underbrace{0, \ldots, 0}_{q}, \underbrace{n-1, \ldots, n-1}_{q}) \notin \mathrm{R}_{\mathbf{b}}^{\mathbf{a}}$.

Let $i, j \in\{1, \ldots, p\}$ with $i \neq j$. Then

$$
(\underbrace{0, \ldots, 0, \stackrel{i}{0}, 0 \ldots, 0, n-1,0, \ldots, 0}_{p}, \underbrace{0, \ldots, 0}_{q}) \in \mathrm{R}_{\mathbf{b}}^{\mathbf{a}}=d_{\theta} .
$$

Thus $(i, j) \notin \theta$. Let $i, j \in\{1, \ldots, q\}$ with $i \neq j$. Then

$$
(\underbrace{n-1, \ldots, n-1}_{p}, \underbrace{0, \ldots, 0, \stackrel{i+p}{0}, 0, \ldots, 0, n^{j+p}-1,0, \ldots, 0}_{q}) \in \mathrm{R}_{\mathbf{b}}^{\mathbf{a}}=d_{\theta} .
$$

Thus $(i+p, j+p) \notin \theta$. Let $i \in\{1, \ldots, p\}$ and $j \in\{1, \ldots, q\}$. Then

$$
(\underbrace{n-1, \ldots, n-1, n-1, n-1, \ldots, n-1}_{p}, \underbrace{0, \ldots, 0, \stackrel{i+p}{0}, 0, \ldots, 0}_{q}) \in \mathrm{R}_{\mathbf{b}}^{\mathbf{a}}=d_{\theta} .
$$

Thus $(i, j+p) \notin \theta$.

Hence, if $i, j \in\{1, \ldots, p+q\}$ with $(i, j) \in \theta$ then $i=j$. This shows $\theta=\Delta_{\mathbf{m}}$. 
An example of a non-trivial clausal relation can be found in 2.1.3 b).

Let us end this section with a lemma that shows under which conditions a nontrivial clausal relation is contained in other non-trivial clausal relation.

2.2.9 Lemma. Let $p, q \in \mathbb{N}_{+}, \mathbf{a}=\left(a_{1}, \ldots, a_{p}\right), \mathbf{a}^{\prime}=\left(a_{1}^{\prime}, \ldots, a_{p}^{\prime}\right) \in(D \backslash\{0\})^{p}$, and $\mathbf{b}=\left(b_{1}, \ldots, b_{q}\right), \mathbf{b}^{\prime}=\left(b_{1}^{\prime}, \ldots, b_{q}^{\prime}\right) \in(D \backslash\{n-1\})^{q}$. Then

$$
\mathrm{R}_{\mathrm{b}^{\prime}}^{\mathrm{a}^{\prime}} \subseteq \mathrm{R}_{\mathrm{b}}^{\mathrm{a}}
$$

holds, if and only if $a_{i}^{\prime} \geq a_{i}$ for all $i \in\{1, \ldots, p\}$ and $b_{j}^{\prime} \leq b_{j}$ for all $j \in\{1, \ldots, q\}$.

Proof. $\Rightarrow$ : Let $\mathrm{R}_{\mathbf{b}^{\prime}}^{\mathbf{a}^{\prime}} \subseteq \mathrm{R}_{\mathbf{b}}^{\mathbf{a}}$ and let $\mathbf{r}:=\left(0, \ldots, 0, a_{i}^{\prime}, 0, \ldots, 0, n-1, \ldots, n-1\right)$ with $i \in\{1, \ldots, p\}$. Obviously, $\mathbf{r} \in \mathrm{R}_{\mathbf{b}^{\prime}}^{\mathbf{a}^{\prime}}$. Since $\mathbf{R}_{\mathbf{b}^{\prime}}^{\mathbf{a}^{\prime}} \subseteq \mathrm{R}_{\mathbf{b}}^{\mathbf{a}}$ we get

$$
0 \geq a_{1} \vee \ldots \vee a_{i}^{\prime} \geq a_{i} \vee \ldots \vee 0 \geq a_{p} \vee n-1 \leq b_{1} \vee \ldots \vee n-1 \leq b_{q}
$$

Hence, $a_{i}^{\prime} \geq a_{i}$ because $0 \nsupseteq a_{i}$ for $i \in\{1, \ldots, p\}$, and $n-1 \not \leq b_{j}$ for $j \in\{1, \ldots, q\}$. Similarly, we obtain $b_{j}^{\prime} \leq b_{j}$ for $j \in\{1, \ldots, q\}$ if we consider the tuple

$$
\left(0, \ldots, 0, n-1, \ldots, n-1, b_{j}, n-1, \ldots, n-1\right) .
$$

Conversely, let $(\mathbf{x}, \mathbf{y})=\left(x_{1}, \ldots, x_{p}, y_{1}, \ldots, y_{q}\right) \in \mathbf{R}_{\mathbf{b}^{\prime}}^{\mathbf{a}^{\prime}}$, i.e.

$$
x_{1} \geq a_{1}^{\prime} \vee \ldots \vee x_{p} \geq a_{p}^{\prime} \vee y_{1} \leq b_{1}^{\prime} \vee \ldots \vee y_{q} \leq b_{q}^{\prime} .
$$

If $x_{i} \geq a_{i}^{\prime}$ for some $i \in\{1, \ldots, p\}$, then because $a_{i}^{\prime} \geq a_{i}$ for all $i \in\{1, \ldots, p\}$, we obtain $x_{i} \geq a_{i}$. Otherwise, because of the expression above $y_{j} \leq b_{j}^{\prime}$ for some $j \in\{1, \ldots, q\}$ and since $b_{j}^{\prime} \leq b_{j}$ for all $j \in\{1, \ldots, q\}$, we get $y_{j} \leq b_{j}$. Thus, $(\mathbf{x}, \mathbf{y}) \in \mathrm{R}_{\mathbf{b}}^{\mathbf{a}}$. 


\section{$3 C$-clones}

In this chapter we study a restricted version of the Galois connection Pol - Inv; where the relations are clausal relations. This restriction provide us with a smaller number of Galois closed sets, a fact that motivated us to describe them.

\subsection{Galois connection $\mathrm{Pol}-C$ Inv}

Next we present a restriction of the well-known Galois connection Pol - Inv where the relations are confined to be clausal relations.

3.1.1 Definition. For $F \subseteq \mathrm{O}_{D}$ we define $C \operatorname{Inv} F:=\operatorname{Inv} F \cap C \mathrm{R}_{D}$. The operators

$$
C \operatorname{Inv}: \mathcal{P}\left(\mathrm{O}_{D}\right) \longrightarrow \mathcal{P}\left(C \mathrm{R}_{D}\right): \quad F \mapsto C \operatorname{Inv} F
$$

and

$$
\mathrm{Pol}: \mathcal{P}\left(C \mathrm{R}_{D}\right) \longrightarrow \mathcal{P}\left(\mathrm{O}_{D}\right): \quad Q \mapsto \operatorname{Pol} Q
$$

define a Galois connection $\mathrm{Pol}-C$ Inv, induced by the relation $\triangleright$, between sets of operations and sets of clausal relations.

We call the Galois closed sets of operations and relations of this Galois connection $C$-clones and relational $C$-clones, respectively. They are defined more formally as follows.

3.1.2 Definition. A set $F \subseteq \mathrm{O}_{D}$ of operations is called a $C$-clone if $F=\operatorname{Pol} Q$ for some set $Q \subseteq C \mathrm{R}_{D}$ of clausal relations, and a set $Q \subseteq C \mathrm{R}_{D}$ is called relational $C$-clone if $Q=C \operatorname{Inv} F$ for a set $F$ of operations.

Every Galois connection naturally gives rise to a pair of closure operators, for the ones belonging to Definition 3.1.1, we introduce a special notation.

3.1.3 Definition. For any $F \subseteq \mathrm{O}_{D}$ and any $Q \subseteq \mathrm{R}_{D}$ we set

$$
\begin{aligned}
& \langle F\rangle_{\mathcal{C}}:=\operatorname{Pol} C \operatorname{Inv} F, \\
& {[Q]_{\mathcal{C}}:=C \operatorname{Inv} \operatorname{Pol} Q .}
\end{aligned}
$$

The following easy and useful observation shows that every $C$-clone is determined by a set of non-trivial clausal relations. 
3.1.4 Remark. For $Q \subseteq C \mathrm{R}_{D}$, it follows that $\operatorname{Pol}(Q)=\operatorname{Pol}\left(Q \cap C \mathrm{R}_{D}^{*}\right)$.

Proof. Let $Q \subseteq C \mathrm{R}_{D}$. We have $Q \cap C \mathrm{R}_{D}^{*} \subseteq Q$, hence $\operatorname{Pol} Q \subseteq \operatorname{Pol}\left(Q \cap C \mathrm{R}_{D}^{*}\right)$. The other inclusion is true because $Q \backslash C \mathrm{R}_{D}^{*} \subseteq\left\{D^{p+q} \mid p, q \in \mathbb{N}_{+}\right\}$contains only trivial relations, and $f \triangleright D^{p+q}$ for any $f \in \mathrm{O}_{D}$.

Let us finish this section with a lemma explaining the relationship between the closure operator $\langle F\rangle_{\mathcal{C}}$ and the clone generation, i.e. the corresponding closure operator of the Galois connection Pol - Inv.

3.1.5 Lemma. For any $F \subseteq \mathrm{O}_{D}$ it follows that:

(a) $\langle F\rangle_{O_{D}} \subseteq\langle F\rangle_{\mathcal{C}}$.

(b) $\left\langle\langle F\rangle_{\mathcal{C}}\right\rangle_{O_{D}}=\langle F\rangle_{\mathcal{C}}$, in particular every $C$-clone is a clone.

Proof. The first statement follows from $C \operatorname{Inv} F \subseteq \operatorname{Inv} F$ for any $F \subseteq \mathrm{O}_{D}$, hence $\langle F\rangle_{O_{D}}=\operatorname{Pol} \operatorname{Inv} F \subseteq \operatorname{Pol} C \operatorname{Inv} F=\langle F\rangle_{\mathcal{C}}$. For the second statement observe that Pol Inv $\langle F\rangle_{\mathcal{C}}=\langle F\rangle_{\mathcal{C}}$.

Let $C L_{D}=\left\{\operatorname{Pol} Q \mid Q \subseteq C \mathrm{R}_{D}\right\}$ be the set of all $C$-clones on $D$. We denote by $\mathcal{C} \mathcal{L}_{D}:=\left(C L_{D}, \subseteq\right)$ the lattice of all $C$-clones on $D$. The greatest element of this lattice is $\mathrm{O}_{D}$ and smallest element is $\mathrm{Pol} C \mathrm{R}_{D}$.

If the lattice $\mathcal{C} \mathcal{L}_{D}=\left(C L_{D}, \subseteq\right)$ is finite, then it can be presented in a Hasse diagram. ${ }^{1}$ In such a drawing, vertices correspond to $C$-clones and two vertices are joined by a line segment exactly if the element corresponding to the vertex drawn higher on the page covers the element corresponding to the vertex drawn lower on the page. Here $a$ covers $b$ means that $a \supset b$ but $a \supset c \supset b$ holds for no $c$.

Our main goal is to analyse the lattice of all $C$-clones on $D$, the first step, is to describe the lattice of $C$-clones for $D=\{0,1\}$.

\subsection{Boolean $C$-clones}

In this section we describe the lattice of all Boolean $C$-clones, i.e. when $D$ is the set $\{0,1\}$ that we also denote by $\mathbf{2}$ for short.

From Lemma 2.2.8, the set $C \mathrm{R}_{\mathbf{2}}$ can be partitioned as in the following corollary.

3.2.1 Corollary. The set $C \mathrm{R}_{\mathbf{2}}$ can be partitioned as

$$
C \mathrm{R}_{\mathbf{2}}=\left\{\mathbf{2}^{p+q} \mid p, q \in \mathbb{N}_{+}\right\} \dot{\cup} C \mathrm{R}_{\mathbf{2}}^{*},
$$

where

$$
C \mathrm{R}_{\mathbf{2}}^{*}=\left\{\mathrm{R}_{\mathbf{0}, q}^{\mathbf{1}, p} \mid p, q \in \mathbb{N}_{+}\right\}
$$

\footnotetext{
${ }^{1}$ More information about Hasse diagrams can be found in [DP02] and [GW99].
} 
and

$$
\mathrm{R}_{\mathbf{0}, q}^{\mathbf{1}, p}:=R_{(\underbrace{(\overbrace{1, \ldots, 1}^{p \text { times }}}_{\underbrace{(0, \ldots, 0)}_{q \text { times }}})} .
$$

Observe that

$$
\begin{aligned}
\mathrm{R}_{\mathbf{0}, q}^{\mathbf{1}, p} & =\left\{(\mathbf{x}, \mathbf{y}) \in \mathbf{2}^{p} \times \mathbf{2}^{q} \mid \exists i \in\{1, \ldots, p\}: x_{i}=1 \vee \exists j \in\{1, \ldots, q\}: y_{j}=0\right\} \\
& =\mathbf{2}^{p+q} \backslash\{(0, \ldots, 0,1, \ldots, 1)\}
\end{aligned}
$$

The remark to come, which follows from 3.1.4, shows that every Boolean $C$-clone can be determined by sets of non-trivial clausal relations on 2 .

\subsubsection{Remark. For $Q \subseteq C \mathrm{R}_{\mathbf{2}}$, it follows that $\operatorname{Pol}(Q)=\operatorname{Pol}\left(Q \cap C \mathrm{R}_{\mathbf{2}}^{*}\right)$.}

We shall describe $\left\{\operatorname{Pol} Q \mid Q \subseteq C \mathrm{R}_{2}\right\}$. Since Pol $-C$ Inv is a Galois connection, this set is dually isomorphic to $\left\{C \operatorname{Inv} F \mid F \subseteq \mathrm{O}_{2}\right\}$. Furthermore, we have

$$
C \operatorname{Inv} F=\bigcap_{f \in F} C \operatorname{Inv} f
$$

for $F \subseteq \mathrm{O}_{2}$. Consequently, it suffices to regard the closed relational sets $C \operatorname{Inv} f$ for $f \in \mathrm{O}_{2}$. Since there is a one to one correspondence between $C \operatorname{Inv} f$ and $\langle f\rangle_{\mathcal{C}}$ via the operators Pol and $C$ Inv, we first consider one-generated $C$-clones. By Remark 3.2.2 and Definition 3.1.3,

$$
\langle f\rangle_{\mathcal{C}}=\operatorname{Pol} C \operatorname{Inv} f=\operatorname{Pol}\left(C \operatorname{Inv} f \cap C \mathrm{R}_{\mathbf{2}}^{*}\right),
$$

i.e. $\langle f\rangle_{\mathcal{C}}$ is the set of all the functions that preserve all the non-trivial invariant clausal relations of $f$.

For the rest of this section we are going to characterise, one-generated $C$-clones for some special functions $f \in \mathrm{O}_{2}$, (namely $f \in\{\neg, h, \vee, \wedge, g\}$ ). We also use the notation from Fig. 3.1 after Lemma 3.2.10, without further explanation.

3.2.3 Lemma. Let $\neg: \mathbf{2} \longrightarrow \mathbf{2}$ be the negation operation, i.e. $\neg(0)=1$ and $\neg(1)=0$. Then we have

$$
\langle\neg\rangle_{\mathcal{C}}=\mathrm{O}_{2}
$$

Proof. By Definition 3.1.3 and Remark 3.2.2, we have

$$
\langle\neg\rangle_{\mathcal{C}}=\left\{f \in \mathrm{O}_{\mathbf{2}} \mid \forall p, q \in \mathbb{N}_{+}: \quad \neg \triangleright \mathrm{R}_{\mathbf{0}, q}^{\mathbf{1 , p}} \Longrightarrow f \triangleright \mathrm{R}_{\mathbf{0}, q}^{\mathbf{1}, p}\right\}
$$

Because of (3.1) it is enough to show $\neg \not \mathbf{R}_{\mathbf{0}, q}^{\mathbf{1 , p}}$ for all $p, q \in \mathbb{N}_{+}$. Indeed, the tuple $\mathbf{r}=(1, \ldots, 1,0, \ldots, 0)$ belongs to $\mathbf{R}_{\mathbf{0}, q}^{\mathbf{1}, p}=\mathbf{2}^{p+q} \backslash\{(0, \ldots, 0,1, \ldots, 1)\}$ for all $p, q \in \mathbb{N}_{+}$, but $\neg[\mathbf{r}]=(\neg(1), \ldots, \neg(1), \neg(0), \ldots, \neg(0))=(0, \ldots, 0,1, \ldots, 1) \notin \mathbf{R}_{\mathbf{0}, q}^{\mathbf{1}, p}$. 
Note that for $p=q=1$ it holds

$$
\mathrm{R}_{\mathbf{0}, q}^{\mathbf{1}, p}=\mathrm{R}_{0}^{1}=\{(0,0),(1,0),(1,1)\}=\geqq,
$$

and hence

$$
\operatorname{Pol}\left(\mathrm{R}_{0}^{1}\right)=\operatorname{Pol}(\geqq)=\operatorname{Pol}(\leqq)=M,
$$

where $M$ is the clone of all monotone Boolean functions.

3.2.4 Lemma. Let $h \in \mathrm{O}_{\mathbf{2}}^{(3)}$ be the ternary majority function on $\mathbf{2}$ (median), i.e. $h(x, y, z):=(x \wedge y) \vee(x \wedge z) \vee(y \wedge z)$ for $x, y, z \in \mathbf{2}$. Then we have

$$
\langle h\rangle_{\mathcal{C}}=\operatorname{Pol}\left(\mathrm{R}_{0}^{1}\right) .
$$

Proof. We show that $h \not \mathbf{R}_{\mathbf{0}, q}^{\mathbf{1}, p}$, unless $p=1, q=1$. We consider several cases:

- $p \geq 2, q \geq 1$ : The scheme

$$
\begin{aligned}
& h(1 \quad 0 \quad 0)=0 \\
& h\left(\begin{array}{lll}
0 & 1 & 0
\end{array}\right)=0 \\
& h\left(\begin{array}{lll}
0 & 0 & 0
\end{array}\right)=0 \\
& h\left(\begin{array}{lll}
0 & 0 & 0
\end{array}\right)=0 \quad(\text { row } p) \\
& h\left(\begin{array}{lll}
1 & 1 & 0
\end{array}\right)=1 \\
& h\left(\begin{array}{lll}
1 & 1 & 1
\end{array}\right)=1 \\
& h\left(\begin{array}{lll}
1 & 1 & 1
\end{array}\right)=1 \quad(\text { row } p+q)
\end{aligned}
$$

shows that $h \not \mathbf{R}_{\mathbf{0}, q}^{\mathbf{1}, p}$, because the tuples (columns of the arguments of $h$ ) all belong to $\mathrm{R}_{\mathbf{0}, q}^{\mathbf{1}, p}$, but after applying $h$ to the tuples, one obtains a tuple (column) that does not belong to $\mathrm{R}_{\mathbf{0}, q}^{\mathbf{1}, p}$.

- $p \geq 1, q \geq 2$ : Likewise, the scheme

$$
\begin{aligned}
& h\left(\begin{array}{lll}
1 & 0 & 0
\end{array}\right)=0 \\
& h\left(\begin{array}{lll}
0 & 0 & 0
\end{array}\right)=0 \\
& h\left(\begin{array}{lll}
0 & 0 & 0
\end{array}\right)=0 \quad(\text { row } p) \\
& h\left(\begin{array}{lll}
1 & 0 & 1
\end{array}\right)=1 \\
& h\left(\begin{array}{lll}
1 & 1 & 0
\end{array}\right)=1 \\
& h\left(\begin{array}{lll}
1 & 1 & 1
\end{array}\right)=1 \\
& h\left(\begin{array}{lll}
1 & 1 & 1
\end{array}\right)=1 \quad(\text { row } p+q)
\end{aligned}
$$

shows that $h \not \mathrm{R}_{\mathbf{0}, q}^{\mathbf{1}, p}$. 
If $p=q=1$, then $h \triangleright \mathrm{R}_{0}^{1}$, because $h$ is a monotone operation and $M=\operatorname{Pol}\left(\mathrm{R}_{0}^{1}\right)$.

3.2.5 Lemma. Let $\wedge$ and $\vee$ be the binary operators, logical conjunction and logical disjunction, respectively. Then we have

$$
\begin{aligned}
& \langle\wedge\rangle_{\mathcal{C}}=\operatorname{Pol}\left\{\mathrm{R}_{\mathbf{0}, q}^{\mathbf{1}, p} \mid p=1, q \in \mathbb{N}_{+}\right\}, \\
& \langle\vee\rangle_{\mathcal{C}}=\operatorname{Pol}\left\{\mathrm{R}_{\mathbf{0}, q}^{\mathbf{1}, p} \mid q=1, p \in \mathbb{N}_{+}\right\} .
\end{aligned}
$$

Proof. At first we have a look at $\wedge$ :

We show that $\wedge \not \mathrm{R}_{\mathbf{0}, q}^{\mathbf{1}, p}$, unless $p=1$ and $q \in \mathbb{N}_{+}$. We consider two cases:

- $p \geq 2, q \in \mathbb{N}_{+}$: The scheme

$$
\begin{array}{rrrr}
1 \wedge & =0 & \\
0 \wedge 1 & \wedge & \\
0 \wedge 0 & =0 & \\
& \vdots & & \\
0 \wedge 0 & =0 & (\text { row } p) \\
1 \wedge 1 & =1 & \\
& \vdots & & \\
1 \wedge & =1 & (\text { row } p+q)
\end{array}
$$

shows that $\wedge \not \mathrm{R}_{\mathbf{0}, q}^{1, p}$.

- $p=1, q \in \mathbb{N}_{+}$: We show

$$
\wedge \triangleright \mathrm{R}_{0, q}^{1, p}
$$

We assume the existence of tuples

$$
\left(x_{1}, y_{1}, \ldots, y_{q}\right),\left(x_{2}, z_{1}, \ldots, z_{q}\right) \in \mathrm{R}_{\mathbf{0}, q}^{\mathbf{1}, p}
$$

such that

$$
x_{1} \wedge x_{2}=0
$$

and for all $j \in\{1, \ldots, q\}$

$$
y_{j} \wedge z_{j}=1 \text {. }
$$

Because of $x_{1} \wedge x_{2}=0$ w.l.o.g. $x_{1}=0$. Then our assumption

$$
\left(x_{1}, y_{1}, \ldots, y_{q}\right) \in \mathbf{R}_{\mathbf{0}, q}^{\mathbf{1}, p}
$$

implies that there is one $j \in\{1, \ldots, q\}$ such that $y_{j}=0$. Thus, $y_{j} \wedge z_{j}=0$, a contradiction.

Similarly, the result for $\vee$ can be proved.

In 3.2.1 we saw that clausal relations are either total or total without one tuple, and none of the diagonals except total relations are clausal relations. Furthermore, $C \operatorname{Inv} \mathrm{O}_{2}=\operatorname{Inv} \mathrm{O}_{2} \cap C \mathrm{R}_{2}=\operatorname{diag}(\mathbf{2}) \cap C \mathrm{R}_{2}$, hence we obtain the following lemma. 
3.2.6 Lemma. The following equality holds

$$
C \operatorname{Inv} \mathrm{O}_{\mathbf{2}}=\left\{\mathbf{2}^{(p+q)} \mid p, q \in \mathbb{N}_{+}\right\} .
$$

Observe that the unary constant operations $c_{0}, c_{1}$ on $\mathbf{2}$, preserve all non-trivial clausal relations on 2 . That is, because $c_{0}\left(y_{1}\right)=0$ and $c_{1}\left(x_{1}\right)=1$ for any tuple $\left(x_{1}, \ldots, x_{p}, y_{1}, \ldots, y_{q}\right)$ of $\mathrm{R}_{\mathbf{0}, q}^{\mathbf{1}, p}$, i.e. For any $p, q \in \mathbb{N}_{+}$we have

$$
c_{0} \triangleright \mathrm{R}_{\mathbf{0}, q}^{\mathbf{1}, p} \text { and } c_{1} \triangleright \mathrm{R}_{\mathbf{0}, q}^{\mathbf{1}, p} \text {. }
$$

In the rest of the section we will freely use $\vee$ to denote supremum of two clones in Post's Lattice (see Fig. 3.1). Nevertheless, we hope not to confuse the reader and be clear.

3.2.7 Lemma. The smallest Boolean $C$-clone is

$$
\langle\emptyset\rangle_{\mathcal{C}}=\operatorname{Pol}\left(C \mathrm{R}_{\mathbf{2}}\right)=\left\{f \in \mathrm{O}_{\mathbf{2}} \mid \forall p, q \in \mathbb{N}_{+}: f \triangleright \mathrm{R}_{\mathbf{0}, q}^{\mathbf{1}, p}\right\} .
$$

It holds

$$
\langle\emptyset\rangle_{\mathcal{C}} \supseteq\left\langle c_{0}\right\rangle_{\mathrm{O}_{2}} \vee\left\langle c_{1}\right\rangle_{\mathrm{O}_{2}} \stackrel{\text { Fig.3.1 }}{=}\left\langle c_{0}, c_{1}\right\rangle_{\mathrm{O}_{2}}
$$

and

$$
\begin{array}{r}
\langle\emptyset\rangle_{\mathcal{C}} \varsubsetneqq\langle\wedge\rangle_{\mathcal{C}} \varsubsetneqq M, \\
\langle\emptyset\rangle_{\mathcal{C}} \varsubsetneqq\langle\vee\rangle_{\mathcal{C}} \varsubsetneqq M .
\end{array}
$$

Furthermore, $\langle\wedge\rangle_{\mathcal{C}}$ and $\langle\vee\rangle_{\mathcal{C}}$ are incomparable $C$-clones.

Proof. From the previous observation we obtain $c_{0}, c_{1} \in\langle\emptyset\rangle_{\mathcal{C}}$. Because $\langle\emptyset\rangle_{\mathcal{C}}$ is a Boolean clone, we have

$$
\left\langle c_{0}\right\rangle_{\mathrm{O}_{2}},\left\langle c_{1}\right\rangle_{\mathrm{O}_{2}} \subseteq\left\langle\langle\emptyset\rangle_{\mathcal{C}}\right\rangle_{\mathrm{O}_{2}} \stackrel{3.1 .5}{=}\langle\emptyset\rangle_{\mathcal{C}}
$$

Thus, $\langle\emptyset\rangle_{\mathcal{C}}$ is an upper bound for $\left\langle c_{0}\right\rangle_{\mathrm{O}_{2}}$ and $\left\langle c_{1}\right\rangle_{\mathrm{O}_{2}}$. Hence,

$$
\left\langle c_{0}\right\rangle_{\mathrm{O}_{2}} \vee\left\langle c_{1}\right\rangle_{\mathrm{O}_{2}} \subseteq\langle\emptyset\rangle_{\mathcal{C}} .
$$

Because $\langle\emptyset\rangle_{\mathcal{C}}=\operatorname{Pol}\left(C \mathrm{R}_{2}\right)$, we have that neither $\wedge \in\langle\emptyset\rangle_{\mathcal{C}}$ nor $\vee \in\langle\emptyset\rangle_{\mathcal{C}}$, because $\wedge \not \mathrm{R}_{\mathbf{0}, q}^{\mathbf{1}, p}$ for $p \geq 2$ and $\vee \not \mathrm{R}_{\mathbf{0}, q}^{\mathbf{1}, p}$ for $q \geq 2$. Thus,

$$
\begin{aligned}
& \wedge \in\langle\wedge\rangle_{\mathcal{C}} \backslash\langle\emptyset\rangle_{\mathcal{C}} \\
& \vee \in\langle\vee\rangle_{\mathcal{C}} \backslash\langle\emptyset\rangle_{\mathcal{C}} .
\end{aligned}
$$

Lemma 3.2.4 implies $M=\operatorname{Pol}\left(\mathrm{R}_{0}^{1}\right)$. Thence, (cf. 3.2.5)

$$
\langle\wedge\rangle_{\mathcal{C}},\langle\vee\rangle_{\mathcal{C}} \subseteq M \stackrel{3.2 .4}{=}\langle h\rangle_{\mathcal{C}} .
$$

This inclusion is proper since

$$
h \in\left(M \backslash\langle\wedge\rangle_{\mathcal{C}}\right) \cap\left(M \backslash\langle\vee\rangle_{\mathcal{C}}\right)
$$


This holds because $h$ is a monotone operation and $h \not \mathbf{R}_{\mathbf{0}, q}^{\mathbf{1}, p}$ for $p=1, q>1$ and for $q=1, p>1$.

Because of $\wedge \not \mathrm{R}_{\mathbf{0}, q}^{\mathbf{1}, p}$ for $p \geq 2$ and $q=1$, we have $\wedge \in\langle\wedge\rangle_{\mathcal{C}} \backslash\langle\vee\rangle_{\mathcal{C}}$, and because of $\vee \not \mathrm{R}_{\mathbf{0}, q}^{\mathbf{1}, p}$ for $p=1$ and $q \geq 2$ we have $\vee \in\langle\vee\rangle_{\mathcal{C}} \backslash\langle\wedge\rangle_{\mathcal{C}}$. Consequently, the two $C$-clones are incomparable.

3.2.8 Lemma. For any subset $F \subseteq \mathrm{O}_{2}$ it follows that

$$
\left\langle c_{0}, c_{1}\right\rangle_{\mathrm{O}_{2}} \vee\langle F\rangle_{\mathrm{O}_{2}} \subseteq\langle F\rangle_{\mathcal{C}}
$$

Proof. From 3.2.7 we infer

$$
\left\langle c_{0}, c_{1}\right\rangle_{\mathrm{O}_{2}} \subseteq\langle\emptyset\rangle_{\mathcal{C}} \subseteq\langle F\rangle_{\mathcal{C}}
$$

Because of $\langle F\rangle_{\mathcal{C}} \in \mathcal{L}_{\mathbf{2}}$ and $F \subseteq\langle F\rangle_{\mathcal{C}}$ we have

$$
\langle F\rangle_{\mathrm{O}_{2}} \subseteq\left\langle\langle F\rangle_{\mathcal{C}}\right\rangle_{\mathrm{O}_{2}} \stackrel{3.1 .5}{=}\langle F\rangle_{\mathcal{C}}
$$

Consequently, we obtain $\left\langle c_{0}, c_{1}\right\rangle_{\mathrm{O}_{2}} \vee\langle F\rangle_{\mathrm{O}_{2}} \subseteq\langle F\rangle_{\mathcal{C}}$.

3.2.9 Lemma. The following equalities hold

$$
\left\langle c_{0}, c_{1}, \wedge\right\rangle_{\mathrm{O}_{2}}=\langle\wedge\rangle_{\mathcal{C}} \quad \text { and } \quad\left\langle c_{0}, c_{1}, \vee\right\rangle_{\mathrm{O}_{2}}=\langle\vee\rangle_{\mathcal{C}}
$$

Proof. From 3.2.8 and Fig. 3.1 we obtain

$$
\left\langle c_{0}, c_{1}, \wedge\right\rangle_{\mathrm{O}_{2}} \stackrel{\text { Fig.3.1 }}{=}\left\langle c_{0}, c_{1}\right\rangle_{\mathrm{O}_{2}} \vee\langle\wedge\rangle_{\mathrm{O}_{2}} \subseteq\langle\wedge\rangle_{\mathcal{C}}
$$

Let us assume

$$
\left\langle c_{0}, c_{1}, \wedge\right\rangle_{\mathrm{O}_{2}} \varsubsetneqq\langle\wedge\rangle_{\mathcal{C}}
$$

Then, $\langle\wedge\rangle_{\mathcal{C}}$ has to be a clone in $\mathcal{L}_{2}$ being above $\left\langle c_{0}, c_{1}, \wedge\right\rangle_{\mathrm{O}_{2}}$. Because the upper cover of $\left\langle c_{0}, c_{1}, \wedge\right\rangle_{\mathrm{O}_{2}}$ in $\mathcal{L}_{2}$ is $M$ (see Post's Lattice, Fig. 3.1), it follows

$$
M \subseteq\langle\wedge\rangle_{\mathcal{C}}
$$

which is a contradiction to $M \supset\langle\wedge\rangle_{\mathcal{C}}$ (cf. Lemma 3.2.7). Similarly, the claim for $\langle\vee\rangle_{\mathcal{C}}$ can be proved.

The lemma to come is valid not only for $D=\mathbf{2}$, but also for $D=\{0,1, \ldots, n-1\}$.

3.2.10 Lemma. For any two subsets $F, G \subseteq \mathrm{O}_{D}$ the following implication holds

$$
F \subseteq G \subseteq\langle F\rangle_{\mathcal{C}} \Longrightarrow\langle G\rangle_{\mathcal{C}}=\langle F\rangle_{\mathcal{C}}
$$

Proof. Since \langle\rangle$_{\mathcal{C}}$ is a closure operator, we obtain

$$
\begin{aligned}
F \subseteq G & \Longrightarrow\langle F\rangle_{\mathcal{C}} \subseteq\langle G\rangle_{\mathcal{C}} \text { and } \\
G \subseteq\langle F\rangle_{\mathcal{C}} & \Longrightarrow\langle G\rangle_{\mathcal{C}} \subseteq\left\langle\langle F\rangle_{\mathcal{C}}\right\rangle_{\mathcal{C}}=\langle F\rangle_{\mathcal{C}} .
\end{aligned}
$$




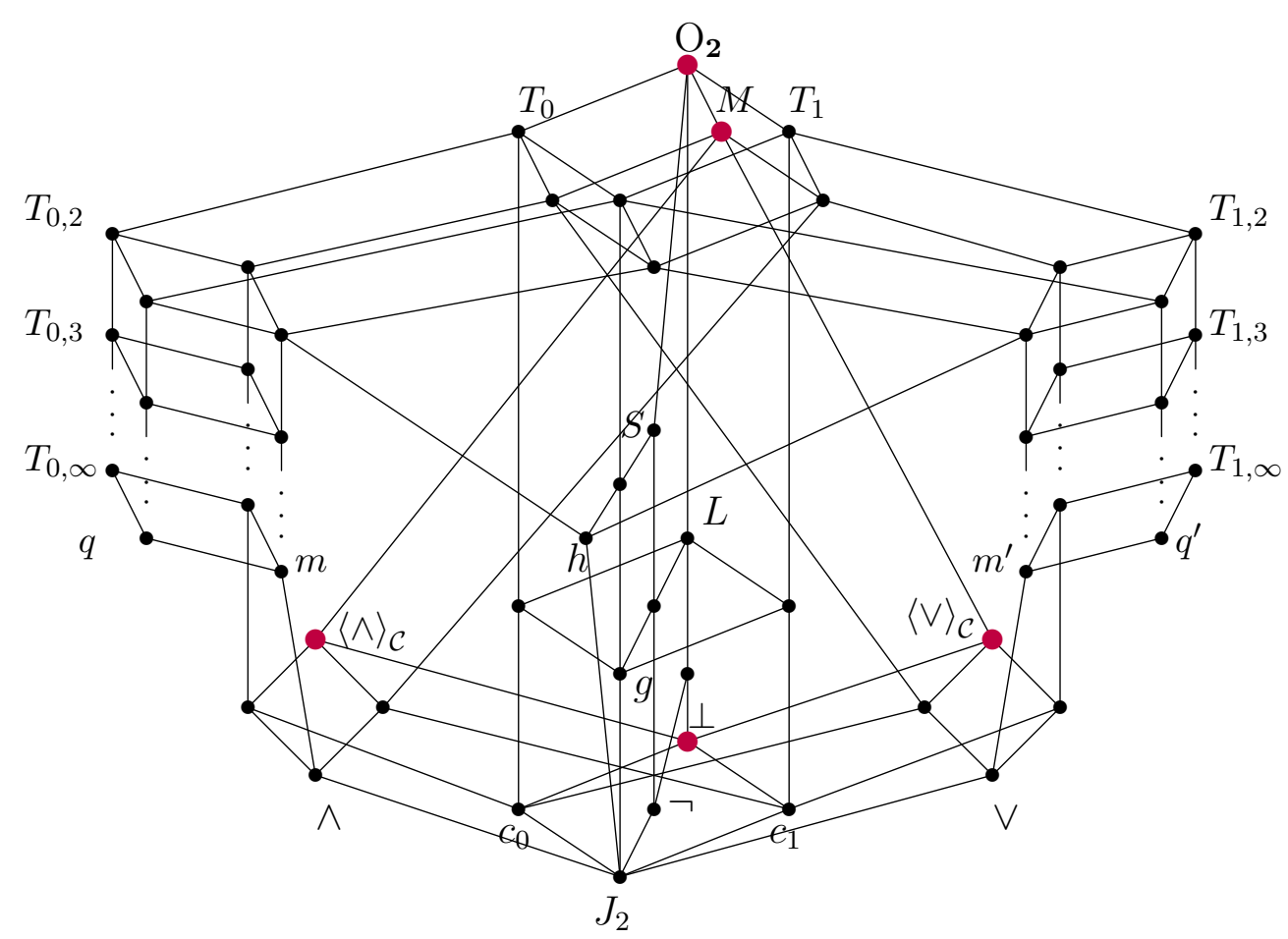

Figure 3.1: Post's Lattice

3.2.11 Lemma. Let $c_{0}, c_{1}$ be the unary constant operations on $\mathbf{2}$ with values 0 and 1 , respectively. Then it follows that

$$
\langle\emptyset\rangle_{\mathcal{C}}=\left\langle c_{0}, c_{1}\right\rangle_{\mathrm{O}_{2}}
$$

Proof. It holds,

$$
\langle\emptyset\rangle_{\mathcal{C}} \subseteq\langle\vee\rangle_{\mathcal{C}} \cap\langle\wedge\rangle_{\mathcal{C}} \stackrel{3.2 .9}{=}\left\langle c_{0}, c_{1}, \wedge\right\rangle_{\mathrm{O}_{2}} \cap\left\langle c_{0}, c_{1}, \vee\right\rangle_{\mathrm{O}_{2}} \stackrel{\text { Fig.3.1 }}{=}\left\langle c_{0}, c_{1}\right\rangle_{\mathrm{O}_{2}} \stackrel{3.2 .7}{\subseteq}\langle\emptyset\rangle_{\mathcal{C}} .
$$

3.2.12 Lemma. Let $g$ be the ternary minority operation, i.e.

$$
g(x, x, y)=g(x, y, x)=g(y, x, x)=y .
$$

Then we have the following equalities

$$
\langle g\rangle_{\mathcal{C}}=\langle L\rangle_{\mathcal{C}}=\mathrm{O}_{2}
$$

Proof. Because of $\neg \in L$, it follows that

$$
\mathrm{O}_{\mathbf{2}} \stackrel{3.2 .3}{=}\langle\neg\rangle_{\mathcal{C}} \subseteq\langle L\rangle_{\mathcal{C}} \subseteq \mathrm{O}_{\mathbf{2}}
$$

hence $\langle L\rangle_{\mathcal{C}}=\mathrm{O}_{\mathbf{2}}$. Applying Lemma 3.2.8 to $\{g\}$ leads to

$$
\{g\} \subseteq L \stackrel{\text { Fig.3.1 }}{=}\left\langle c_{0}, c_{1}\right\rangle_{\mathrm{O}_{2}} \vee\langle g\rangle_{\mathrm{O}_{2}} \subseteq\langle g\rangle_{\mathcal{C}} .
$$


Together with 3.2 .10 we infer

$$
\langle L\rangle_{\mathcal{C}}=\langle g\rangle_{\mathcal{C}}
$$

3.2.13 Remark. Let $F \leq \mathrm{O}_{2}$ be a clone in Post's Lattice. Then we have

$$
\mathcal{C L}_{\mathbf{2}}=\left\{\operatorname{Pol}(C \operatorname{Inv}(F)) \mid F \subseteq \mathrm{O}_{\mathbf{2}}\right\}=\left\{\operatorname{Pol}(C \operatorname{Inv}(F)) \mid F \leq \mathrm{O}_{\mathbf{2}}\right\}
$$

Proof. It is obvious that

$$
\left\{\operatorname{Pol}(C \operatorname{Inv}(F)) \mid F \leq \mathrm{O}_{2}\right\} \subseteq\left\{\operatorname{Pol}(C \operatorname{Inv}(F)) \mid F \subseteq \mathrm{O}_{2}\right\}
$$

To show the other inclusion we regard $F \subseteq \mathrm{O}_{2}$. It holds for $G:=\operatorname{Pol} \operatorname{Inv}(F) \leq \mathrm{O}_{2}$

$$
\begin{aligned}
\operatorname{Pol} C \operatorname{Inv}(G) & =\operatorname{Pol}\left((\operatorname{Inv}(G)) \cap C \mathrm{R}_{D}\right)=\operatorname{Pol}\left((\operatorname{Inv}(\operatorname{Pol} \operatorname{Inv}(F))) \cap C \mathrm{R}_{D}\right) \\
& =\operatorname{Pol}\left(\operatorname{Inv}(F) \cap C \mathrm{R}_{D}\right)=\operatorname{Pol} C \operatorname{Inv}(F) .
\end{aligned}
$$

The theorem below, given also in [Var10], states that there are no more Boolean $C$-clones than the ones already described in the previous Lemmata 3.2.4, 3.2.9, 3.2.11, 3.2.12.

3.2.14 Theorem. The lattice of all Boolean $C$-clones is

$$
\mathcal{C} \mathcal{L}_{2}=\left\{\perp,\langle\wedge\rangle_{\mathcal{C}},\langle\vee\rangle_{\mathcal{C}},\langle h\rangle_{\mathcal{C}}, \mathrm{O}_{2}\right\}
$$

where

$$
\perp:=\left\langle c_{0}, c_{1}\right\rangle_{\mathrm{O}_{2}}, \quad\langle\wedge\rangle_{\mathcal{C}}=\left\langle c_{0}, c_{1}, \wedge\right\rangle_{\mathrm{O}_{2}}, \quad\langle\vee\rangle_{\mathcal{C}}=\left\langle c_{0}, c_{1}, \vee\right\rangle_{\mathrm{O}_{2}}, \quad \text { and }\langle h\rangle_{\mathcal{C}}=M
$$

Proof. The next six equalities are consequences of Lemma 3.2.10 and previous lemmata.

$$
\begin{aligned}
\left\{\langle C\rangle_{\mathcal{C}} \mid C \in\left[J_{2},\left\langle c_{0}, c_{1}\right\rangle_{\mathrm{O}_{2}}\right]_{\mathcal{L}_{2}}\right\} & =\left\{\left\langle c_{0}, c_{1}\right\rangle_{\mathrm{O}_{2}}\right\} \\
\left\{\langle C\rangle_{\mathcal{C}} \mid C \in\left[\langle\wedge\rangle_{\mathrm{O}_{2}},\left\langle\wedge, c_{0}, c_{1}\right\rangle_{\mathrm{O}_{2}}\right]_{\mathcal{L}_{2}}\right\} & =\left\{\left\langle\wedge, c_{0}, c_{1}\right\rangle_{\mathrm{O}_{2}}\right\} \\
\left\{\langle C\rangle_{\mathcal{C}} \mid C \in\left[\langle\vee\rangle_{\mathrm{O}_{2}},\left\langle\vee, c_{0}, c_{1}\right\rangle_{\mathrm{O}_{2}}\right]_{\mathcal{L}_{2}}\right\} & =\left\{\left\langle\vee, c_{0}, c_{1}\right\rangle_{\mathrm{O}_{2}}\right\} \\
\left\{\langle C\rangle_{\mathcal{C}} \mid C \in\left[\langle\neg\rangle_{\mathrm{O}_{2}}, \mathrm{O}_{2}\right]_{\mathcal{L}_{2}}\right\} & =\left\{\mathrm{O}_{2}\right\} \\
\left\{\langle C\rangle_{\mathcal{C}} \mid C \in\left[\langle g\rangle_{\mathrm{O}_{2}}, \mathrm{O}_{2}\right]_{\mathcal{L}_{2}}\right\} & =\left\{\mathrm{O}_{2}\right\} \\
\left\{\langle C\rangle_{\mathcal{C}} \mid C \in\left[\langle h\rangle_{\mathrm{O}_{2}}, M\right]_{\mathcal{L}_{2}}\right\} & =\{M\}
\end{aligned}
$$


The next four equalities will be shown below.

$$
\begin{aligned}
\left\{\langle C\rangle_{\mathcal{C}} \mid C \in\left[\langle\wedge\rangle_{\mathrm{O}_{2}}, M\right]_{\mathcal{L}_{2}} \backslash\left[\langle\wedge\rangle_{\mathrm{O}_{2}},\left\langle\wedge, c_{0}, c_{1}\right\rangle_{\mathrm{O}_{2}}\right]_{\mathcal{L}_{2}}\right\} & =\{M\} \\
\left\{\langle C\rangle_{\mathcal{C}} \mid C \in\left[\langle\wedge\rangle_{\mathrm{O}_{2}}, \mathrm{O}_{2}\right]_{\mathcal{L}_{2}} \backslash\left[\langle\wedge\rangle_{\mathrm{O}_{2}},\left\langle\wedge, c_{0}, c_{1}\right\rangle_{\mathrm{O}_{2}}\right]_{\mathcal{L}_{2}}, C \nsubseteq M\right\} & =\left\{\mathrm{O}_{2}\right\} \\
\left\{\langle C\rangle_{\mathcal{C}} \mid C \in\left[\langle\vee\rangle_{\mathrm{O}_{2}}, M\right]_{\mathcal{L}_{2}} \backslash\left[\langle\vee\rangle_{\mathrm{O}_{2}},\left\langle\vee, c_{0}, c_{1}\right\rangle_{\mathrm{O}_{2}}\right]_{\mathcal{L}_{2}}\right\} & =\{M\} \\
\left\{\langle C\rangle_{\mathcal{C}} \mid C \in\left[\langle\vee\rangle_{\mathrm{O}_{2}}, \mathrm{O}_{2}\right]_{\mathcal{L}_{2}} \backslash\left[\langle\vee\rangle_{\mathrm{O}_{2}},\left\langle\vee, c_{0}, c_{1}\right\rangle_{\mathrm{O}_{2}}\right]_{\mathcal{L}_{2}}, C \nsubseteq M M\right. & =\left\{\mathrm{O}_{2}\right\}
\end{aligned}
$$

Regarding a clone $C \leq \mathrm{O}_{2}$ with $\langle\wedge\rangle_{\mathrm{O}_{2}} \subseteq C$, but $C \notin\left[\langle\wedge\rangle_{\mathrm{O}_{2}},\left\langle\wedge, c_{0}, c_{1}\right\rangle_{\mathrm{O}_{2}}\right]_{\mathcal{L}_{2}}$ yields (using Lemma 3.2.8)

$$
C_{1}:=\left(\left\langle c_{0}, c_{1}\right\rangle_{\mathrm{O}_{2}} \vee C\right) \subseteq\langle C\rangle_{\mathcal{C}} .
$$

If $C \subseteq M$, then one can read off Post's Lattice that $M=C_{1}$. Furthermore, because of monotonicity of $\langle\cdot\rangle_{\mathcal{C}}$ yields $\langle C\rangle_{\mathcal{C}} \subseteq\langle M\rangle_{\mathcal{C}}=M$. Thus,

$$
\langle C\rangle_{\mathcal{C}}=M
$$

Otherwise, (i. e. $C \nsubseteq M$ ) leads to

$$
\mathrm{O}_{2}=C_{1} \subseteq\langle C\rangle_{\mathcal{C}} \subseteq \mathrm{O}_{2} .
$$

The proof for $\vee$ instead of $\wedge$ is similar. Knowing all these 10 equalities and applying Remark 3.2.13, one obtains

$$
\mathcal{C} \mathcal{L}_{2}=\left\{\perp,\langle\wedge\rangle_{\mathcal{C}},\langle\vee\rangle_{\mathcal{C}}, M, \mathrm{O}_{2}\right\}
$$

the clones which are described in the previous lemmata.

The previous theorem does not only describe the set of all the Boolean $C$-clones but also the operations that these contain. For example, $\langle\vee\rangle_{\mathcal{C}}$ contains the operations $c_{0}, c_{1}, \vee$, all the projections and compositions of these functions.

As we mentioned, when we describe $C$-clones at the same time we are describing relational $C$-clones. The lattices of Boolean $C$-clones and of Boolean relational $C$-clones are dually isomorphic as is shown in the next figure.

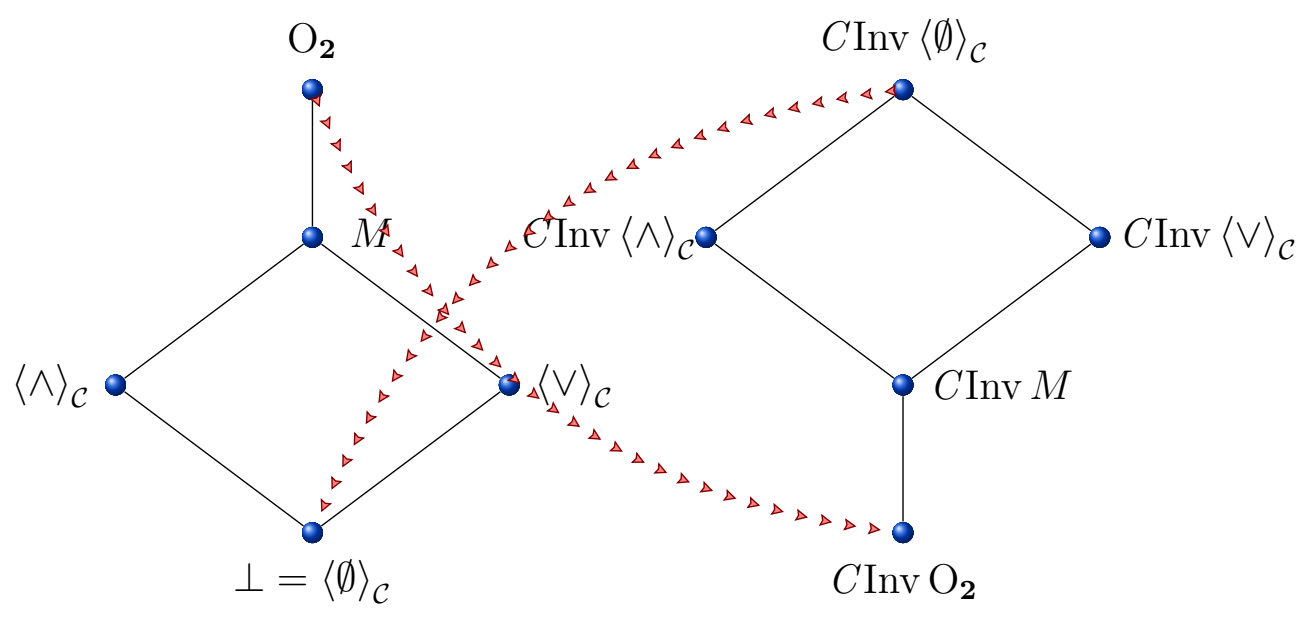


For the remainder of this section, we restrict ourselves to the following: Given a $C$-clone $\operatorname{Pol}(Q)$ with $Q \subseteq C \mathrm{R}_{\mathbf{2}}^{*}$, find a minimal subset $Q_{1} \subseteq Q$, such that

$$
\operatorname{Pol}(Q)=\operatorname{Pol}\left(Q_{1}\right)
$$

The motivation for the restriction is to establish that all Boolean $C$-clones can be described by a finite number of clausal relations.

\subsubsection{Lemma. The following equalities hold}

$$
\begin{aligned}
\mathrm{O}_{\mathbf{2}} & =\operatorname{Pol}(\emptyset) \\
M & =\operatorname{Pol}\left(\mathrm{R}_{0}^{1}\right) \\
\langle\wedge\rangle_{\mathcal{C}} & =\operatorname{Pol}\left\{\mathrm{R}_{\mathbf{0}, q}^{\mathbf{1 , p}} \mid p=1, q \in \mathbb{N}_{+}\right\}=\operatorname{Pol}\left(\mathrm{R}_{(0,0)}^{1}\right), \\
\langle\vee\rangle_{\mathcal{C}} & =\operatorname{Pol}\left\{\mathrm{R}_{\mathbf{0}, q}^{\mathbf{1}, p} \mid q=1, p \in \mathbb{N}_{+}\right\}=\operatorname{Pol}\left(\mathrm{R}_{0}^{(1,1)}\right), \\
\left\langle c_{0}, c_{1}\right\rangle_{\mathrm{O}_{\mathbf{2}}} & =\operatorname{Pol}\left\{\mathrm{R}_{\mathbf{0}, q}^{\mathbf{1}, p} \mid p, q \in \mathbb{N}_{+}\right\}=\operatorname{Pol}\left(\mathrm{R}_{(0,0)}^{(1,1)}\right) .
\end{aligned}
$$

Proof. The characterization of $\mathrm{O}_{2}$ is trivial. The second statement follows from Lemma 3.2.4. The arguments for the rest of the equalities are very similar, so w.l.o.g. we will only deal with the characterization of $\langle\wedge\rangle_{\mathcal{C}}$.

"ᄃ": We have

$$
\left\{\mathrm{R}_{(0,0)}^{1,}\right\} \subseteq\left\{\mathrm{R}_{\mathbf{0}, q}^{\mathbf{1}, p} \mid p=1, q \in \mathbb{N}_{+}\right\}
$$

hence

$$
W:=\operatorname{Pol}\left\{\mathrm{R}_{(0,0)}^{1}\right\} \supseteq \operatorname{Pol}\left\{\mathrm{R}_{\mathbf{0}, q}^{\mathbf{1}, p} \mid p=1, q \in \mathbb{N}_{+}\right\}=\langle\wedge\rangle_{\mathcal{C}} .
$$

"": $\quad$ From the proof of Lemma 3.2.4 we know $M=\langle h\rangle_{\mathcal{C}} \nsubseteq W$, applying the above-established facts that

$$
\left\langle c_{0}, c_{1}, \wedge\right\rangle_{\mathrm{O}_{2}} \stackrel{3.2 .9}{=}\langle\wedge\rangle_{\mathcal{C}} \subseteq W
$$

and $C$-clones are clones, one can read off of Post's Lattice that

$$
W=\left\langle c_{0}, c_{1}, \wedge\right\rangle_{\mathrm{O}_{2}}=\langle\wedge\rangle_{\mathcal{C}}
$$

To finish this section, let us summarize the results obtained so far in two tables. Below, we show clausal relations that are preserved by functions $f \in \mathrm{O}_{2}$, (namely $\left.f \in\left\{\wedge, \vee, \neg, c_{0}, c_{1}, h, g\right\}\right)$. These functions generate atoms in the Post's Lattice. 
TABLE 1

\begin{tabular}{|c|c|c|c|c|c|}
\hline$f$ & & $f \triangleright \mathrm{R}_{\mathbf{0}, q}^{1, p}$ & & $f \not \mathrm{R}_{\mathbf{0}, q}^{1, p}$ & $\langle f\rangle_{\mathcal{C}}$ \\
\hline$\wedge$ & $\mathrm{R}_{\mathbf{0}, q}^{\mathbf{1}, p}$ & for $p=1, q \in \mathbb{N}_{+}$ & $\mathbf{R}_{\mathbf{0}, q}^{\mathbf{1}, p}$ & for $p \geq 2, q \in \mathbb{N}_{+}$ & $\langle\wedge\rangle_{\mathcal{C}}=\left\langle c_{0}, c_{1}, \wedge\right\rangle_{\mathrm{O}_{2}}$ \\
\hline V & $\mathrm{R}_{\mathbf{0}, q}^{\mathbf{1 , p}}$ & for $p \in \mathbb{N}_{+}, q=1$ & $\mathrm{R}_{\mathbf{0}, q}^{\mathbf{1 , p}}$ & for $p \in \mathbb{N}_{+}, q \geq 2$ & $\mid\langle\vee\rangle_{\mathcal{C}}=\left\langle c_{0}, c_{1}, \vee\right\rangle_{\mathrm{O}_{2}}$ \\
\hline$\neg$ & & & $\mathrm{R}_{\mathbf{0}, q}^{\mathbf{1}, p}$ & for $p, q \in \mathbb{N}_{+}$ & $\langle\neg\rangle_{\mathcal{C}}=\mathrm{O}_{2}$ \\
\hline$c_{0}$ & $\mathrm{R}_{\mathbf{0}, q}^{\mathbf{1}, p}$ & for $p, q \in \mathbb{N}_{+}$ & & & $c_{0} \in\langle\emptyset\rangle_{\mathcal{C}}$ \\
\hline$c_{1}$ & $\mathrm{R}_{\mathbf{0}, q}^{1, p}$ & for $p, q \in \mathbb{N}_{+}$ & & & $c_{1} \in\langle\emptyset\rangle_{\mathcal{C}}$ \\
\hline$h$ & $\mathrm{R}_{\mathbf{0}, q}^{\mathbf{1}, p}$ & for $p=1, q=1$ & $\mathrm{R}_{\mathbf{0}, q}^{\mathbf{1 , p}}$ & for $(p, q) \neq(1,1)$ & $\langle h\rangle_{\mathcal{C}}=M$ \\
\hline$g$ & & & $\mathbf{R}_{\mathbf{0}, q}^{\mathbf{1 , p}}$ & for $p, q \in \mathbb{N}_{+}$ & $\langle g\rangle_{\mathcal{C}}=\mathrm{O}_{2}$ \\
\hline
\end{tabular}

In the following table, we give minimal subsets $Q_{i}$ of clausal relations to determine every Boolean $C$-clone.

TABLE 2

\begin{tabular}{|c|c|c|}
\hline$C$-clones & $C \operatorname{Inv}\left(C_{i}\right) \backslash\left\{D^{p+q} \mid p, q \in \mathbb{N}_{+}\right\}$ & $C_{i}=\operatorname{Pol} Q_{i}$ \\
where $1 \leq i \leq 6$ & where $Q_{i} \in C \mathrm{R}_{D}^{*}$ \\
\hline$C_{1}=\langle\emptyset\rangle_{\mathcal{C}}=\left\langle c_{0}, c_{1}\right\rangle_{\mathrm{O}_{\mathbf{2}}}$ & $C \operatorname{Inv}\left(C_{1}\right)=\left\{\mathrm{R}_{\mathbf{0}, q}^{\mathbf{1}, p} \mid p=q=2\right\}$ & $\operatorname{Pol}_{(0,0)}^{(1,1)}$ \\
$C_{2}=\langle\wedge\rangle_{\mathcal{C}}=\left\langle c_{0}, c_{1}, \wedge\right\rangle_{\mathrm{O}_{\mathbf{2}}}$ & $C \operatorname{Inv}\left(C_{2}\right)=\left\{\mathrm{R}_{\mathbf{0}, q}^{\mathbf{1}, p} \mid p=1, q=2\right\}$ & $\operatorname{Pol}_{(0,0)}^{1}$ \\
$C_{3}=\langle\vee\rangle_{\mathcal{C}}=\left\langle c_{0}, c_{1}, \vee\right\rangle_{\mathrm{O}_{\mathbf{2}}}$ & $C \operatorname{Inv}\left(C_{3}\right)=\left\{\mathrm{R}_{\mathbf{0}, q}^{\mathbf{1}, p} \mid p=2, q=1\right\}$ & $\operatorname{PolR}_{0}^{(1,1)}$ \\
$C_{4}=\langle h\rangle_{\mathcal{C}}=M$ & $C \operatorname{Inv}\left(C_{4}\right)=\left\{\mathrm{R}_{\mathbf{0}, q}^{\mathbf{1}, p} \mid p=1, q=1\right\}$ & $\operatorname{Pol}_{0}^{1}$ \\
$C_{5}=\langle\neg\rangle_{\mathcal{C}}=\mathrm{O}_{\mathbf{2}}$ & $C \operatorname{Inv}\left(C_{5}\right)=\emptyset$ & $\operatorname{Pol} \emptyset$ \\
$C_{6}=\langle g\rangle_{\mathcal{C}}=\mathrm{O}_{\mathbf{2}}$ & $C \operatorname{Inv}\left(C_{6}\right)=\emptyset$ & $\operatorname{Pol} \emptyset$ \\
\hline
\end{tabular}




\subsection{The duality principle for $C$-clones}

In previous sections properties (statements) of the lower parameters $\mathbf{b}$ of clausal relations $R_{b}^{a}$, could be handled analogously to properties of the upper parameters a. In this section we explain how a statement for the upper parameters a of clausal relations $\mathrm{R}_{\mathrm{b}}^{\mathrm{a}}$ will correspond to a dual statement concerning the lower parameters $\mathbf{b}$.

Let $\alpha: D \longrightarrow D$ be the permutation defined by

$$
\alpha(x):=n-1-x .
$$

We define the dual element of $x \in D$ by $x^{\partial}:=x^{\alpha}:=\alpha(x)=n-1-x$. For every $u, v$ belonging to $D$, it holds

$$
u \leq v \Longleftrightarrow u^{\alpha} \geq v^{\alpha}
$$

Furthermore, $\left(v^{\alpha}\right)^{\alpha}=v$, i.e. $\alpha^{-1}=\alpha$. Since (3.2) and because $\alpha$ is an isomorphism, we have that $\alpha$ is an order-isomorphism ${ }^{2}$ from $(D, \leq)$ to $(D, \geq)$.

Let $m \in \mathbb{N}_{+}$, for an $m$-ary tuple $\mathbf{x}=\left(x_{1}, \ldots, x_{m}\right)$ on $D^{m}$, we denote by

$$
\mathbf{x}^{\partial}:=\mathbf{x}^{\alpha}:=\left(x_{1}^{\alpha}, x_{2}^{\alpha}, \ldots, x_{m}^{\alpha}\right)=\left(n-1-x_{1}, n-1-x_{2}, \ldots, n-1-x_{m}\right),
$$

the dual m-ary tuple on $D^{m}$. For an $m$-ary relation $\varrho$ on $D$, we associate a relation

$$
\varrho^{\alpha}:=\left\{\mathbf{x}^{\alpha} \mid \mathbf{x} \in \varrho\right\}
$$

and for a set $Q$ of relations on $D$, we let $Q^{\alpha}:=\left\{\varrho^{\alpha} \mid \varrho \in Q\right\}$.

Let $k \in \mathbb{N}_{+}$. The dual of a $k$-ary operation $f$ on $D$ is the $k$-ary operation $f^{\partial}:=f^{\alpha}$, where

$$
\begin{aligned}
f^{\alpha}(\mathbf{x}) & :=\left(f\left(\mathbf{x}^{\alpha^{-1}}\right)\right)^{\alpha}=\left(f\left(\mathbf{x}^{\alpha}\right)\right)^{\alpha} \\
& =n-1-f\left(n-1-x_{1}, \ldots, n-1-x_{k}\right) .
\end{aligned}
$$

It is not difficult to verify that

$$
\begin{aligned}
f\left(\mathbf{x}^{\alpha}\right) & =\left(\left(f\left(\mathbf{x}^{\alpha}\right)\right)^{\alpha}\right)^{\alpha}=\left(f^{\alpha}(\mathbf{x})\right)^{\alpha}, \\
\left(f^{\alpha}\right)^{\alpha}(\mathbf{x}) & =f(\mathbf{x})=\left(f^{\alpha}\left(\mathbf{x}^{\alpha}\right)\right)^{\alpha} .
\end{aligned}
$$

To every $F \subseteq \mathrm{O}_{D}$, we assign $F^{\partial}=F^{\alpha}:=\left\{f^{\alpha} \mid f \in F\right\}$ as the set of dual operations on $D$. In what follows we need the following lemma and remark.

3.3.1 Lemma. Let $f \in \mathrm{O}_{D}$ and let $\varrho \in \mathrm{R}_{D}$. Then it follows that

$$
f \triangleright \varrho \Longleftrightarrow f^{\alpha} \triangleright \varrho^{\alpha} .
$$

\footnotetext{
${ }^{2}$ In this work some notions such as order-isomorphism or linearly ordered set will be not defined explicitly. A nice introduction to these concepts can be found in [DP02].
} 
Proof. Let $k, m$ be positive natural numbers, $f \in \mathrm{O}_{D}^{(k)}$ and let $\varrho \in \mathrm{R}_{D}^{(m)}$. First, we show $f \triangleright \varrho$ implies $f^{\alpha} \triangleright \varrho^{\alpha}$. Consider any $m$-ary tuples $\mathbf{r}_{1}^{\alpha}, \ldots \mathbf{r}_{k}^{\alpha}$ belonging to $\varrho^{\alpha}$, where $\mathbf{r}_{l}^{\alpha}=\left(x_{1 l}^{\alpha}, x_{2 l}^{\alpha}, \ldots, x_{m l}^{\alpha}\right)$ for $l \in\{1, \ldots, k\}$. Since $\mathbf{r}_{1}^{\alpha}, \ldots \mathbf{r}_{k}^{\alpha} \in \varrho^{\alpha}$, we obtain that $\mathbf{r}_{1}, \ldots \mathbf{r}_{k} \in \varrho$. Because $f \triangleright \varrho$, we get $f\left[\mathbf{r}_{1}, \ldots \mathbf{r}_{k}\right] \in \varrho$. Hence,

$$
\begin{aligned}
f\left[\mathbf{r}_{1}, \ldots \mathbf{r}_{k}\right] \stackrel{(3.5)}{=}\left(\left(f^{\alpha}\left(x_{11}^{\alpha}, x_{12}^{\alpha}, \ldots, x_{1 k}^{\alpha}\right)\right)^{\alpha}, \ldots,\left(f^{\alpha}\left(x_{m 1}^{\alpha}, x_{m 2}^{\alpha}, \ldots, x_{m k}^{\alpha}\right)\right)^{\alpha}\right) \in \varrho
\end{aligned}
$$

The latter implies $\left(f^{\alpha}\left(x_{11}^{\alpha}, x_{12}^{\alpha}, \ldots, x_{1 k}^{\alpha}\right), \ldots, f^{\alpha}\left(x_{m 1}^{\alpha}, x_{m 2}^{\alpha}, \ldots, x_{m k}^{\alpha}\right)\right) \in \varrho^{\alpha}$ and hence $f^{\alpha}\left[\mathbf{r}_{1}^{\alpha}, \ldots \mathbf{r}_{k}^{\alpha}\right] \in \varrho^{\alpha}$. Conversely, let $f^{\alpha} \triangleright \varrho^{\alpha}$, using the first part of this proof, we get

$$
f=\left(f^{\alpha}\right)^{\alpha} \triangleright\left(\varrho^{\alpha}\right)^{\alpha}=\varrho .
$$

Let $F$ be a set of operations on $D$ and let $Q$ be a set of relations on $D$. We define

$$
\operatorname{Pol}^{\alpha}(Q):=\left(\operatorname{Pol} Q^{\alpha}\right)^{\alpha} \text { and } \operatorname{Inv}^{\alpha}(F):=\left(\operatorname{Inv} F^{\alpha}\right)^{\alpha} .
$$

3.3.2 Remark. Let $F \subseteq \mathrm{O}_{D}$ and $Q \subseteq \mathrm{R}_{D}$. Then

$$
\operatorname{Pol}^{\alpha}(Q)=\operatorname{Pol} Q \quad \text { and } \quad \operatorname{Inv}^{\alpha}(F)=\operatorname{Inv} F .
$$

Proof. By definition $\operatorname{Pol}^{\alpha}(Q)=\left(\operatorname{Pol} Q^{\alpha}\right)^{\alpha}$, and hence

$$
\begin{aligned}
\mathrm{Pol}^{\alpha}(Q) & =\left(\mathrm{Pol} Q^{\alpha}\right)^{\alpha}=\left(\left\{f \in \mathrm{O}_{D} \mid \forall \varrho^{\alpha} \in Q^{\alpha}: f \triangleright \varrho^{\alpha}\right\}\right)^{\alpha} \\
& =\left(\left\{f \in \mathrm{O}_{D} \mid \forall \varrho \in Q: f \triangleright \varrho^{\alpha}\right\}\right)^{\alpha} \stackrel{3.3 .1}{=}\left(\left\{f \in \mathrm{O}_{D} \mid \forall \varrho \in Q: f^{\alpha} \triangleright \varrho\right\}\right)^{\alpha} \\
& =\left\{f^{\alpha} \in \mathrm{O}_{D} \mid \forall \varrho \in Q: f^{\alpha} \triangleright \varrho\right\}=\left\{g \in \mathrm{O}_{D} \mid \forall \varrho \in Q: g \triangleright \varrho\right\}=\operatorname{Pol}(Q) .
\end{aligned}
$$

Similarly, one can prove $\operatorname{Inv}^{\alpha}(F)=\operatorname{Inv} F$.

Note that for every tuple a that belongs to $(D \backslash\{0\})^{p}$, its dual $\mathbf{a}^{\alpha}$ belongs to $(D \backslash\{n-1\})^{p}$. Similarly, for $\mathbf{b} \in(D \backslash\{n-1\})^{q}$, we get $\mathbf{b}^{\alpha} \in(D \backslash\{0\})^{q}$.

Let $\mathrm{R}_{\mathbf{b}}^{\mathbf{a}} \in C \mathrm{R}_{(D, \leq)}$ be a clausal relation of arity $p+q$, with respect to the linearly ordered set $(D, \leq)$. That is

$$
\mathrm{R}_{\mathbf{b}}^{\mathbf{a}}=\left\{(\mathbf{x}, \mathbf{y}) \in D^{p+q} \mid x_{1} \geq a_{1} \vee \ldots \vee x_{p} \geq a_{p} \vee y_{1} \leq b_{1} \vee \ldots \vee y_{q} \leq b_{q}\right\} .
$$

Using (3.3) above, we see that $\left(\mathrm{R}_{\mathbf{b}}^{\mathbf{a}}\right)^{\alpha}$ is not a clausal relation with respect to $(D, \leq)$, but w.r.t. $(D, \geq)$. i.e.

$$
\begin{aligned}
\left(\mathrm{R}_{\mathbf{b}}^{\mathbf{a}}\right)^{\alpha} & =\left\{(\mathbf{u}, \mathbf{v}) \in D^{p+q} \mid u_{1} \leq a_{1}^{\alpha} \vee \ldots \vee u_{p} \leq a_{p}^{\alpha} \vee v_{1} \geq b_{1}^{\alpha} \vee \ldots \vee v_{q} \geq b_{q}^{\alpha}\right\} \\
& =\mathrm{R}_{\mathbf{b}^{\alpha}}^{\mathbf{a}^{\alpha}} \text { w.r.t. }(D, \geq) .
\end{aligned}
$$

Next we define an operator $P_{p+q}: \mathrm{R}_{D} \longrightarrow \mathrm{R}_{D}$, which swaps the first $p$ coordinates of every tuple belonging to the relation $\varrho$ with the last $q$ coordinates. That is,

$$
P_{p+q}(\varrho):=\left\{(\mathbf{y}, \mathbf{x}) \in D^{q+p} \mid(\mathbf{x}, \mathbf{y}) \in \varrho\right\} .
$$


Note that for $\pi \in \mathrm{S}_{p+q}$ defined by

$$
\pi(k):= \begin{cases}k+q & \text { if } k \leq p \\ k-p & \text { if } k>p\end{cases}
$$

we have $P_{p+q}(\varrho)=V_{\pi}(\varrho)$.

The operator operates on $\left(\mathrm{R}_{\mathbf{b}}^{\mathbf{a}}\right)^{\alpha}$ as follows

$$
\begin{aligned}
P_{p+q}\left(\left(\mathrm{R}_{\mathbf{b}}^{\mathbf{a}}\right)^{\alpha}\right) & =\left\{(\mathbf{v}, \mathbf{u}) \mid(\mathbf{u}, \mathbf{v}) \in\left(\mathrm{R}_{\mathbf{b}}^{\mathbf{a}}\right)^{\alpha}\right\} \\
& =\left\{(\mathbf{v}, \mathbf{u}) \mid u_{1} \leq a_{1}^{\alpha} \vee \ldots \vee u_{p} \leq a_{p}^{\alpha} \vee v_{1} \geq b_{1}^{\alpha} \vee \ldots \vee v_{q} \geq b_{q}^{\alpha}\right\} \\
& =\left\{(\mathbf{v}, \mathbf{u}) \mid v_{1} \geq b_{1}^{\alpha} \vee \ldots \vee v_{q} \geq b_{q}^{\alpha} \vee u_{1} \leq a_{1}^{\alpha} \vee \ldots \vee u_{p} \leq a_{p}^{\alpha}\right\} \\
& =\mathrm{R}_{\mathbf{a}^{\alpha}}^{\mathbf{b}^{\alpha}} \text { w.r.t. }(D, \leq) .
\end{aligned}
$$

3.3.3 Definition. Let $\mathrm{R}_{\mathbf{b}}^{\mathbf{a}} \in C \mathrm{R}_{(D, \leq)}$ be a clausal relation of arity $p+q$, we define the dual clausal relation of arity $q+p$ by

$$
\left(\mathrm{R}_{\mathbf{b}}^{\mathbf{a}}\right)^{\partial}:=P_{p+q}\left(\left(\mathrm{R}_{\mathbf{b}}^{\mathbf{a}}\right)^{\alpha}\right) \stackrel{(3.7)}{=} \mathrm{R}_{\mathbf{a}^{\alpha}}^{\mathbf{b}^{\alpha}}
$$

The latter implies $P_{p+q}^{-1}\left(\left(\mathrm{R}_{\mathbf{b}}^{\mathbf{a}}\right)^{\partial}\right)=\left(\mathrm{R}_{\mathbf{b}}^{\mathbf{a}}\right)^{\alpha}$. Note also that $\left(\mathrm{R}_{\mathbf{b}}^{\mathbf{a}}\right)^{\partial}=\mathrm{R}_{\mathbf{a}^{\alpha}}^{\mathbf{b}^{\alpha}} \in C \mathrm{R}_{(D, \leq)}$.

The set of clausal relations is defined over a linearly ordered set $(D, \leq)$. From (3.6) we obtain $\left(C \mathrm{R}_{(D, \leq)}\right)^{\alpha}=C \mathrm{R}_{(D, \geq)}$. It follows that the set of duals clausal relations equals

$$
\left(C \mathrm{R}_{(D, \leq)}\right)^{\partial}:=P\left[\left(C \mathrm{R}_{(D, \leq)}\right)^{\alpha}\right] \stackrel{(3.6)}{=} P\left[C \mathrm{R}_{(D, \geq)}\right] \stackrel{(3.7)}{=} C \mathrm{R}_{(D, \leq)} .
$$

Here the notation $P[Q]$ means application of the corresponding operator $P_{p+q}$ to every relation in $Q$.

3.3.4 Lemma. Let $f \in \mathrm{O}_{D}$ and $\mathrm{R}_{\mathbf{b}}^{\mathbf{a}} \in C \mathrm{R}_{D}$. Then it holds

$$
f \triangleright \mathrm{R}_{\mathbf{b}}^{\mathbf{a}} \Longleftrightarrow f^{\partial}=f^{\alpha} \triangleright \mathrm{R}_{\mathbf{a}^{\alpha}}^{\mathbf{b}^{\alpha}}=\left(\mathrm{R}_{\mathbf{b}}^{\mathbf{a}}\right)^{\partial} .
$$

Proof. Let $k$ be a positive natural number and let $f \in \mathrm{O}_{D}^{(k)}$, such that $f \triangleright \mathrm{R}_{\mathbf{b}}^{\mathbf{a}}$. We show $f^{\alpha} \triangleright \mathrm{R}_{\mathbf{a}^{\alpha}}^{\mathbf{b}^{\alpha}}$. Let $\mathbf{r}_{1}, \ldots \mathbf{r}_{k} \in \mathrm{R}_{\mathbf{a}^{\alpha}}^{\mathbf{b}^{\alpha}}$, where

$$
\mathbf{r}_{l}=\left(\mathbf{y}_{l}, \mathbf{x}_{l}\right)=\left(y_{1 l}, \ldots, y_{q l}, x_{1 l}, \ldots, x_{p l}\right)
$$

for $l \in\{1, \ldots, k\}$. Then

$$
\begin{aligned}
& y_{1 l} \geq b_{1}^{\alpha} \vee \ldots \vee y_{q l} \geq b_{q}^{\alpha} \vee x_{1 l} \leq a_{1}^{\alpha} \vee \ldots \vee x_{p l} \leq a_{p}^{\alpha} \\
\stackrel{(3.2)}{\Longleftrightarrow} & x_{1 l}^{\alpha} \geq a_{1} \vee \ldots \vee x_{p l}^{\alpha} \geq a_{p} \vee y_{1 l}^{\alpha} \leq b_{1} \vee \ldots \vee y_{q l}^{\alpha} \leq b_{q} \\
\Longleftrightarrow & \left(x_{1 l}^{\alpha}, \ldots, x_{p l}^{\alpha}, y_{1 l}^{\alpha}, \ldots, y_{q l}^{\alpha}\right) \in \mathrm{R}_{\mathrm{b}}^{\mathrm{a}}
\end{aligned}
$$


Since $f \triangleright \mathrm{R}_{\mathbf{b}}^{\mathbf{a}}$, we get

$$
\begin{gathered}
f\left(x_{11}^{\alpha}, \ldots, x_{1 k}^{\alpha}\right) \geq a_{1} \vee \ldots \vee f\left(x_{p 1}^{\alpha}, \ldots, x_{p k}^{\alpha}\right) \geq a_{p} \vee \\
f\left(y_{11}^{\alpha}, \ldots, y_{1 k}^{\alpha}\right) \leq b_{1} \vee \ldots \vee f\left(y_{q 1}^{\alpha}, \ldots, x_{q k}^{\alpha}\right) \leq b_{q} \\
\stackrel{(3.4)(3.2)}{\Longleftrightarrow} f^{\alpha}\left(y_{11}, \ldots, y_{1 k}\right) \geq b_{1}^{\alpha} \vee \ldots \vee f^{\alpha}\left(y_{q 1}, \ldots, y_{q k}\right) \geq b_{q}^{\alpha} \\
f^{\alpha}\left(x_{11}, \ldots, x_{1 k}\right) \leq a_{1}^{\alpha} \vee \ldots \vee f^{\alpha}\left(x_{p 1}, \ldots, x_{p k}\right) \leq a_{p}^{\alpha} \\
\text { Thus } f^{\alpha}\left[\mathbf{r}_{1}, \ldots \mathbf{r}_{k}\right] \in \mathrm{R}_{\mathbf{a}^{\alpha}}^{\mathbf{b}^{\alpha}} .
\end{gathered}
$$

Let $f^{\alpha} \triangleright \mathrm{R}_{\mathbf{a}^{\alpha}}^{\mathbf{b}^{\alpha}}$, using the above direction, we obtain $f=\left(f^{\alpha}\right)^{\alpha} \triangleright \mathrm{R}_{\left(\mathbf{b}^{\alpha}\right)^{\alpha}}^{\left(\mathbf{a}^{\alpha}{ }^{\alpha}\right.}=\mathrm{R}_{\mathbf{b}}^{\mathbf{a}}$.

We define $C \operatorname{Inv}^{\alpha} F:=\left(C \operatorname{Inv}\left(F^{\alpha}\right)\right)^{\alpha}$. Below we show that $C \operatorname{Inv}^{\alpha} F$ is also a set of invariant clausal relations but w.r.t. $(D, \geq)$.

$$
\begin{aligned}
C \operatorname{Inv}^{\alpha} F & =\left(C \operatorname{Inv}\left(F^{\alpha}\right)\right)^{\alpha}=\left(\left\{\varrho \in C \mathrm{R}_{D} \mid \forall f^{\alpha} \in F^{\alpha}: f^{\alpha} \triangleright \varrho\right\}\right)^{\alpha} \\
& =\left(\left\{\varrho \in C \mathrm{R}_{D} \mid \forall f \in F: f^{\alpha} \triangleright \varrho\right\}\right)^{\alpha} \stackrel{3.3 .4}{=}\left(\left\{\varrho \in C \mathrm{R}_{D} \mid \forall f \in F: f \triangleright \varrho^{\alpha}\right\}\right)^{\alpha} \\
& =\left\{\varrho^{\alpha} \mid \varrho \in C \mathrm{R}_{D} \wedge \forall f \in F: f \triangleright \varrho^{\alpha}\right\}=\left\{\varrho^{\alpha} \mid \varrho \in C \mathrm{R}_{D} \wedge \varrho^{\alpha} \in \operatorname{Inv} F\right\} \\
& =\left\{\varrho^{\alpha} \in\left(C \mathrm{R}_{(D, \leq)}\right)^{\alpha} \mid \varrho^{\alpha} \in \operatorname{Inv} F\right\}=\left\{\sigma \in\left(C \mathrm{R}_{(D, \leq)}\right)^{\alpha} \mid \sigma \in \operatorname{Inv} F\right\} \\
& =\left(C \mathrm{R}_{(D, \leq)}\right)^{\alpha} \cap \operatorname{Inv} F=C \mathrm{R}_{(D, \geq)} \cap \operatorname{Inv} F .
\end{aligned}
$$

Hence,

$$
\begin{aligned}
P\left[C \operatorname{Inv}^{\alpha} F\right] & =P\left[\left(C \mathrm{R}_{(D, \leq)}\right)^{\alpha} \cap \operatorname{Inv} F\right]=P\left[\left(C \mathrm{R}_{(D, \leq)}\right)^{\alpha}\right] \cap P[\operatorname{Inv} F] \\
& =C \mathrm{R}_{D} \cap P[\operatorname{Inv} F]=C \mathrm{R}_{D} \cap \operatorname{Inv} F=C \operatorname{Inv} F \\
(C \operatorname{Inv} F)^{\alpha} & =\left(C \operatorname{Inv}\left(F^{\alpha}\right)^{\alpha}\right)^{\alpha}=C \operatorname{Inv}^{\alpha} F^{\alpha} \stackrel{(3.8)}{=} P^{-1}\left[C \operatorname{Inv} F^{\alpha}\right] \\
(\operatorname{Pol} Q)^{\partial} & =(\operatorname{Pol} Q)^{\alpha} \stackrel{3.3 .2}{=}\left(\operatorname{Pol}^{\alpha} Q\right)^{\alpha}=\left(\left(\operatorname{Pol} Q^{\alpha}\right)^{\alpha}\right)^{\alpha}=\operatorname{Pol} Q^{\alpha}
\end{aligned}
$$

We define

$$
\begin{aligned}
(C \operatorname{Inv} F)^{\partial} & :=P\left[(C \operatorname{Inv} F)^{\alpha}\right] \stackrel{(3.9)}{=} P\left[P^{-1}\left[C \operatorname{Inv} F^{\alpha}\right]\right]=C \operatorname{Inv} F^{\alpha}=C \operatorname{Inv} F^{\partial} . \\
\left(\langle F\rangle_{\mathcal{C}}\right)^{\partial} & =(\operatorname{Pol} C \operatorname{Inv} F)^{\partial}=(\operatorname{Pol} C \operatorname{Inv} F)^{\alpha} \stackrel{(3.10)}{=} \operatorname{Pol}(C \operatorname{Inv} F)^{\alpha} \\
& \stackrel{(3.9)}{=} \operatorname{Pol} P^{-1}\left[C \operatorname{Inv} F^{\alpha}\right]=\operatorname{Pol} C \operatorname{Inv} F^{\alpha}=\operatorname{Pol} C \operatorname{Inv} F^{\partial}=\left\langle F^{\partial}\right\rangle_{\mathcal{C}} .
\end{aligned}
$$

3.3.5 Definition. If $S$ is a statement about clausal relations with respect to the linearly ordered set $(D, \leq)$, then we obtain another statement $S^{\partial}$ about clausal relations with respect to $(D, \leq)$ as follows. Taking $(D, \leq)$ as the canonical representative of an $n$-element chain, we obtain from $S$ a statement $S(L, \sqsubseteq)$ about clausal relations with respect to any linearly ordered set $(L, \sqsubseteq)$, making use of the unique order isomorphism from $(D, \leq)$ onto $(L, \sqsubseteq)$. In particular, for $(L, \sqsubseteq)=(D, \geq)$ we can deduce an intermediate statement $S^{\alpha}: \stackrel{\alpha}{=} S(D, \geq)$ from $S$. Interpreting $S^{\alpha}$ again as a statement about clausal relations with respect to $(D, \leq)$ we obtain the dual statement $S^{\partial}$. 
For example from 3.2.15 we infer

$$
\left\{\wedge, c_{0}, c_{1}\right\}=\langle\wedge\rangle_{\mathcal{C}}=\operatorname{Pol} R_{(0,0)}^{1}
$$

We translate this statement to a dual statement

$$
\begin{aligned}
\left(\langle\wedge\rangle_{\mathcal{C}}\right)^{\partial} & \stackrel{(3.11)}{=}\left\langle\wedge^{\partial}\right\rangle_{\mathcal{C}}=\langle\vee\rangle_{\mathcal{C}} \quad \text { and } \\
\left(\operatorname{PolR}_{(0,0)}^{1}\right)^{\partial} & \stackrel{(3.10)}{=} \operatorname{Pol}\left(\mathrm{R}_{(0,0)}^{1}\right)^{\alpha}=\operatorname{Pol} P_{1+2}^{-1}\left(\mathrm{R}_{(0,0)}^{1}\right)^{\partial}=\operatorname{Pol}\left(\mathrm{R}_{(0,0)}^{1}\right)^{\partial}=\operatorname{Pol} \mathrm{R}_{0}^{(1,1)}
\end{aligned}
$$

Thus, we obtain the dual statement $S^{\partial}:\langle\mathrm{V}\rangle_{\mathcal{C}}=\operatorname{Pol~R}_{0}^{(1,1)}$.

3.3.6 Remark. The last step in dualising a statement $S$ from the intermediate statement $S^{\alpha}$ to the dual statement $S^{\partial}$ is not completely straightforward, since clausal relations with respect to $(D, \geq)$ are generally not clausal relations with respect to $(D, \leq)$, only up to permutation of variables. However, via the mentioned variable permutation they are in one-to-one correspondence to each other. As the relation $\triangleright$ is not affected by variable permutations. This is, for instance, the reason for the second part of Lemma 3.3.4.

3.3.7 Theorem (Duality principle). Let $S$ be a statement about clausal relations with respect to $(D, \leq)$. Then,

$$
S \text { is true } \Longleftrightarrow S^{\partial} \text { is true. }
$$

Proof. It is inherent to the applied method of dualisation that $S$ is true iff $S^{\alpha}$ is true iff $S^{\partial}$ is true.

This duality principle is used to simplify proofs hereafter. If a lemma asserts two statements that are dual to each other, we usually prove only one of them.

\subsection{Some $C$-clones on a finite set.}

In this section we show that clausal relations meet a sufficient condition that is known to ensure polynomial time solvability of the corresponding CSP. Furthermore, in order to analyse the lattice of all $C$-clones we collect some statements that generalise Lemmata $3.2 .4,3.2 .5,3.2 .6$ and 3.2 .8 to the case $|D| \geq 3$. Then, we describe the smallest $C$-clone on a finite set $D$.

3.4.1 Lemma. Let $l \in \mathbb{N}_{+}, c_{0}^{l}, c_{n-1}^{l}$ be l-ary constant operations with values 0 and $n-1$, respectively. Then it follows that

$$
\operatorname{Pol} C \mathrm{R}_{D}=\left\langle\left\{c_{0}^{l}, c_{n-1}^{l}\right\}\right\rangle_{O_{D}}
$$


Proof. First, we show that $\left\langle\left\{c_{0}^{l}, c_{n-1}^{l}\right\}\right\rangle_{O_{D}} \subseteq$ Pol $C \mathrm{R}_{D}$. The set $J_{D}$ of all projections on $D$ belongs to $\mathrm{Pol}_{D} \subseteq \operatorname{Pol} C \mathrm{R}_{D}$. Let $\mathrm{R}_{\mathbf{b}}^{\mathbf{a}} \in C \mathrm{R}_{D}$ with $\mathbf{a} \in D^{p}$ and $\mathbf{b} \in D^{q}$ and $r_{1}, \ldots, r_{l} \in \mathrm{R}_{\mathbf{b}}^{\mathrm{a}}$. Since $0 \leq b_{1}$ and $n-1 \geq a_{1}$, we get

$$
\begin{aligned}
c_{0}^{l}\left[r_{1}, \ldots, r_{l}\right] & =(\stackrel{1}{0}, \ldots, \stackrel{p}{0}, \stackrel{1}{0}, \ldots, \stackrel{q}{0}) \in \mathrm{R}_{\mathbf{b}}^{\mathbf{a}}, \\
\text { and } c_{n-1}^{l}\left[r_{1}, \ldots, r_{l}\right] & =(n \stackrel{1}{-1}, \ldots, n \stackrel{p}{-} 1, n \stackrel{1}{-1}, \ldots, n \stackrel{q}{-} 1) \in \mathrm{R}_{\mathbf{b}}^{\mathbf{a}}
\end{aligned}
$$

Consequently, $c_{0}^{l} \triangleright \mathrm{R}_{\mathbf{b}}^{\mathbf{a}}$ and $c_{n-1}^{l} \triangleright \mathrm{R}_{\mathbf{b}}^{\mathbf{a}}$. Conversely, we assume that for some $k \in \mathbb{N}_{+}$ there is a function

$$
f \in\left(\mathrm{Pol}^{(k)} C \mathrm{R}_{D}\right) \backslash\left\{\left\{c_{0}^{k}, c_{n-1}^{k}\right\} \cup J_{D}\right\}
$$

- Since $f \neq c_{0}^{k}$, there is some $\left(y_{1}^{\prime}, \ldots, y_{k}^{\prime}\right) \in D^{k}$ such that $f\left(y_{1}^{\prime}, \ldots, y_{k}^{\prime}\right)>0$.

- As $f \neq c_{n-1}^{k}$, there is some $\left(x_{1}^{\prime}, \ldots, x_{k}^{\prime}\right) \in D^{k}$ such that $f\left(x_{1}^{\prime}, \ldots, x_{k}^{\prime}\right)<n-1$.

Since $f \notin J_{D}$, we get $f \neq e_{j}^{k}$ for all $j \in\{1, \ldots, k\}$. Hence, for all $j$ belonging to $\{1, \ldots, k\}$ there exists a tuple $\left(d_{1}^{j}, \ldots, d_{j}^{j}, \ldots, d_{k}^{j}\right)$ in $D^{k}$ depending on $j$ such that

$$
f\left(d_{1}^{j}, \ldots, d_{j}^{j}, \ldots, d_{k}^{j}\right) \neq d_{j}^{j}
$$

We write these tuples as rows in the following matrix

$$
\left(\begin{array}{cccc}
d_{1}^{1} & d_{2}^{1} & \ldots & d_{k}^{1} \\
d_{1}^{2} & d_{2}^{2} & \ldots & d_{k}^{2} \\
& & \vdots & \\
d_{1}^{k} & d_{2}^{k} & \ldots & d_{k}^{k}
\end{array}\right)
$$

We can reorder the rows of this matrix in such way that the rows where $(\star)$ is satisfied because of " $<$ " are listed first, followed by the rows where $(\star)$ is satisfied because of " $>$ ". That is, for some permutation $\pi \in \mathrm{S}_{\{1, \ldots, k\}}$, there is $j \in\{1, \ldots, k+1\}$ such that for every $i \in\{1, \ldots, j-1\}$ we get

$$
f\left(d_{1}^{\pi(i)}, \ldots, d_{k}^{\pi(i)}\right)<d_{\pi(i)}^{\pi(i)}
$$

and for every $i \in\{j, \ldots, k\}$ we get $f\left(d_{1}^{\pi(i)}, \ldots, d_{k}^{\pi(i)}\right)>d_{\pi(i)}^{\pi(i)}$. Consider the tuples

$$
r_{1}:=\left(x_{1}^{\prime}, d_{1}^{\pi(1)}, \ldots, d_{1}^{\pi(k)}, y_{1}^{\prime}\right), \ldots, r_{k}:=\left(x_{k}^{\prime}, d_{k}^{\pi(1)}, \ldots, d_{k}^{\pi(k)}, y_{k}^{\prime}\right)
$$

The scheme 


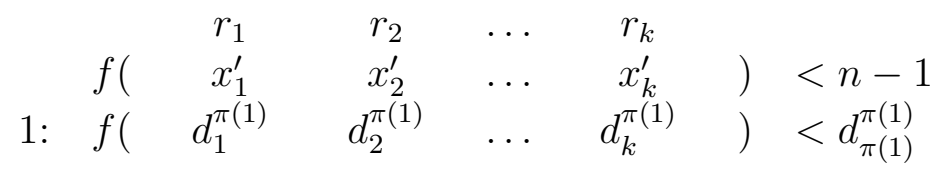

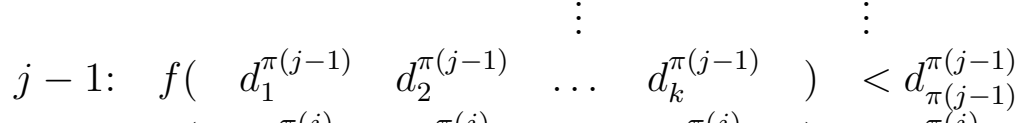

$$
\begin{aligned}
& j: \quad f\left(\begin{array}{lllll}
d_{1}^{\pi(j)} & d_{2}^{\pi(j)} & \ldots & d_{k}^{\pi(j)}
\end{array}\right)>d_{\pi(j)}^{\pi(j)} \\
& f\left(\begin{array}{llll}
d_{1}^{\pi(k)} & d_{2}^{\pi(k)} & \cdots & d_{k}^{\pi(k)}
\end{array}\right)>d_{\pi(k)}^{\pi(k)} \\
& f\left(\begin{array}{ccccc}
y_{1}^{\prime} & y_{2}^{\prime} & \ldots & y_{k}^{\prime}
\end{array}\right)>0
\end{aligned}
$$

shows that $f \not \mathrm{R}_{d_{\pi(j)}^{\pi(j)}, \ldots, d_{\pi(k)}^{\pi(k)}, 0}^{n-1, d_{\pi(1)}^{\pi(1)}, \ldots, d_{\pi-1)}^{\pi(j-1)}}$. This is because the tuples (columns of the arguments of $f$ ) all belong to $\mathrm{R}_{d_{\pi(j)}^{\pi(j)}, \ldots, d_{\pi(k)}^{\pi(k)}, 0}^{n-1, d_{\pi(j-1)}^{\pi(1)}, \ldots, d^{\pi(j-1)}}$, since in the $i$-th column the entry in the $\left(\pi^{-1}(i)+1\right)$-st row is $d_{i}^{i}$, satisfying the clausal expression with equality. However, after applying $f$ to the tuples, one obtains a tuple (column) that does not belong to $\mathrm{R}_{d_{\pi(j)}^{\pi(j)}, \ldots, d_{\pi(k)}^{\pi(k)}, 0}^{n-1, d_{\pi(j-1)}^{\pi(1)}, \ldots, d^{\pi(j-1)}}$. This is impossible, since $f \in \mathrm{Pol}^{(k)} C \mathrm{R}_{D}$.

Consequently, $\left(\mathrm{Pol}^{(k)} C \mathrm{R}_{D}\right) \backslash\left\{\left\{c_{0}^{k}, c_{n-1}^{k}\right\} \cup J_{D}\right\}=\emptyset$ holds for every $k \in \mathbb{N}_{+}$, which proves the claim.

At this point, we know that for every $k \in \mathbb{N}_{+}$the $k$-ary constant operation $c_{0}^{k}$ belongs to $\operatorname{Pol}\left(C \mathrm{R}_{D}\right)$. The latter together with Proposition 1.4.4, make the constraint satisfaction problem over clausal relations, denoted by $\operatorname{CSP}\left(C \mathrm{R}_{D}\right)$, solvable in polynomial time.

Analogously to Lemma 3.2.8, and using the previous lemma, the lemma to come can be proved.

3.4.2 Lemma. For any subset $F \subseteq \mathrm{O}_{D}$ and all $l \in \mathbb{N}_{+}$it follows that

$$
\left\langle\left\{c_{0}^{l}, c_{n-1}^{l}\right\}\right\rangle_{O_{D}} \vee\langle F\rangle_{O_{D}} \subseteq\langle F\rangle_{\mathcal{C}}
$$

In 2.2.8 we saw that clausal relations are either trivial or non-trivial, and none of the diagonals except for the full Cartesian powers are clausal relations. Furthermore, $C \operatorname{Inv} \mathrm{O}_{D}=\operatorname{Inv} \mathrm{O}_{D} \cap C \mathrm{R}_{D}=\operatorname{diag}(D) \cap C \mathrm{R}_{D}$ holds. Hence, we obtain the following lemma.

3.4.3 Lemma. The following equality holds

$$
C \operatorname{Inv} \mathrm{O}_{D}=\left\{D^{p+q} \mid p, q \in \mathbb{N}_{+}\right\}
$$

In the following proofs, we use that the tuple $(n-1, \ldots, n-1,0, \ldots, \stackrel{p}{0})$ belongs to all non-trivial clausal relations $\mathrm{R}_{\mathbf{b}}^{\mathbf{a}}$, but $(0, \ldots, \stackrel{p}{0}, n-1, \ldots, n \stackrel{q}{-} 1)$ does not belong to $\mathrm{R}_{\mathrm{b}}^{\mathrm{a}}$. 
3.4.4 Lemma. Let $h \in \mathrm{O}_{D}^{(3)}$ be the ternary majority operation on $D$ defined by $h(x, y, z):=(x \vee y) \wedge(x \vee z) \wedge(y \vee z)$ where $x \vee y:=\max \{x, y\}$ and $x \wedge y:=\min \{x, y\}$. Then we have

$$
\langle h\rangle_{\mathcal{C}}=\operatorname{Pol}\left\{\mathrm{R}_{b}^{a} \mid a \in D \backslash\{0\}, b \in D \backslash\{n-1\}\right\} .
$$

Proof. Let $\mathrm{R}_{\mathbf{b}}^{\mathbf{a}} \in C \mathrm{R}_{D}^{*}$, i.e. $\mathbf{a} \in(D \backslash\{0\})^{p}$ and $\mathbf{b} \in(D \backslash\{n-1\})^{q}$. We show that $h \not \mathrm{R}_{\mathrm{b}}^{\mathrm{a}}$ unless $p=1, q=1$.

- $p \geq 2, q \geq 1$ : The scheme

$$
\begin{aligned}
& h\left(\begin{array}{ccc}
n-1 & 0 & 0
\end{array}\right)=0 \\
& h\left(\begin{array}{ccc}
0 & n-1 & 0
\end{array}\right)=0 \\
& h\left(\begin{array}{lll}
0 & 0 & 0
\end{array}\right)=0 \\
& h\left(\begin{array}{ccc}
0 & 0 & 0
\end{array}\right)=0 \quad(\text { row } p) \\
& h\left(\begin{array}{ccc}
n-1 & n-1 & 0
\end{array}\right)=n-1 \\
& h(n-1 n-1 n-1)=n-1 \\
& h(n-1 n-1 n-1)=n-1 \quad(\text { row } p+q)
\end{aligned}
$$

shows that $h \not \mathrm{R}_{\mathbf{b}}^{\mathbf{a}}$, because the tuples (columns of the arguments of $h$ ) all belong to $\mathrm{R}_{\mathbf{b}}^{\mathrm{a}}$, but after applying $h$ to the tuples, one obtains a tuple (column) that does not belong to $R_{b}^{a}$.

- $p \geq 1, q \geq 2$ : Likewise, the scheme

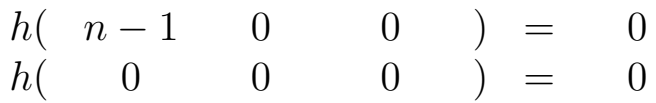

$$
\begin{aligned}
& h\left(\begin{array}{ccc}
0 & 0 & 0
\end{array}\right)=0 \quad(\text { row } p) \\
& h\left(\begin{array}{ccc}
n-1 & 0 & n-1
\end{array}\right)=n-1 \\
& h\left(\begin{array}{ccc}
n-1 & n-1 & 0
\end{array}\right)=n-1 \\
& h(n-1 n-1 n-1)=n-1 \\
& h(n-1 n-1 n-1)=n-1 \quad(\text { row } p+q)
\end{aligned}
$$

- $p=q=1$ : Let $\mathrm{R}_{b}^{a}$ be any non-trivial clausal relation of arity 2, and let $\left(x_{1}, y_{1}\right),\left(x_{2}, y_{2}\right),\left(x_{3}, y_{3}\right) \in \mathrm{R}_{b}^{a}$. We consider two cases:

- Suppose that there exist $i, j \in\{1,2,3\}$ with $i \neq j: x_{i} \geq a, x_{j} \geq a$. Since $h(x, y, z)=(x \vee y) \wedge(x \vee z) \wedge(y \vee z)=(x \wedge y) \vee(x \wedge z) \vee(y \wedge z), h$ is totally symmetric, i.e, $h\left(x_{1}, x_{2}, x_{3}\right)=h\left(x_{\pi(1)}, x_{\pi(2)}, x_{\pi(3)}\right)$ for all $\pi \in \mathrm{S}_{\mathbf{3}}$. Thus, w.l.o.g. we consider $i=1, j=2$, i.e.

$$
x_{1} \geq a, x_{2} \geq a, x_{3} \geq x_{3}
$$


because $h$ is monotone, we obtain $h\left(x_{1}, x_{2}, x_{3}\right) \geq h\left(a, a, x_{3}\right)=a$, and hence

$$
\left(h\left(x_{1}, x_{2}, x_{3}\right), h\left(y_{1}, y_{2}, y_{3}\right)\right) \in \mathrm{R}_{b}^{a}
$$

- Otherwise, there exist $i, j \in\{1,2,3\}$ with $i \neq j: y_{i} \leq b, y_{j} \leq b$, because $\left(x_{1}, y_{1}\right),\left(x_{2}, y_{2}\right),\left(x_{3}, y_{3}\right) \in \mathrm{R}_{b}^{a}$. Furthermore, since $h$ is monotone we get $h\left(y_{1}, y_{2}, y_{3}\right) \leq b$. Thus,

$$
\left(h\left(x_{1}, x_{2}, x_{3}\right), h\left(y_{1}, y_{2}, y_{3}\right)\right) \in \mathrm{R}_{b}^{a} .
$$

The last two cases show $h \triangleright \mathrm{R}_{b}^{a}$.

We finish this section with the next lemma, which characterise $C$-clones generated by the binary operations min and max.

3.4.5 Lemma. Let $x \vee y=\max \{x, y\}$ and $x \wedge y=\min \{x, y\}$ for $x, y \in D$. Then it holds

$$
\begin{aligned}
\langle\wedge\rangle_{\mathcal{C}} & =\operatorname{Pol}\left\{\mathrm{R}_{\mathbf{b}}^{a} \mid a \in D \backslash\{0\}, \mathbf{b} \in(D \backslash\{n-1\})^{q}\right\}, \\
\langle\vee\rangle_{\mathcal{C}} & =\operatorname{Pol}\left\{\mathrm{R}_{b}^{\mathbf{a}} \mid \mathbf{a} \in(D \backslash\{0\})^{p}, b \in D \backslash\{n-1\}\right\} .
\end{aligned}
$$

Proof. At first we look at $\wedge$ : Let $\mathrm{R}_{\mathrm{b}}^{\mathrm{a}} \in C \mathrm{R}_{D}^{*}$. We show that $\wedge \not \mathrm{R}_{\mathrm{b}}^{\mathrm{a}}$, unless when $p=1$ and $q \in \mathbb{N}_{+}$. We consider two cases:

- $p \geq 2$ : The scheme

$$
\begin{aligned}
& n-1 \wedge 0=0 \\
& 0 \wedge n-1=0 \\
& 0 \wedge 0=0 \\
& 0 \wedge 0=0 \quad(\text { row } p) \\
& n-1 \wedge n-1=n-1 \\
& n-1 \wedge n-1=n-1 \quad(\text { row } p+q)
\end{aligned}
$$

shows that $\wedge \not \mathrm{R}_{\mathrm{b}}^{\mathrm{a}}$.

- $p=1, q \in \mathbb{N}_{+}$: We show $\wedge \triangleright \mathrm{R}_{\mathbf{b}}^{a}$ for all $\mathrm{R}_{\mathbf{b}}^{a} \in C \mathrm{R}_{D}^{*}$. We assume the existence of tuples $\left(x_{1}, y_{1}, \ldots, y_{q}\right),\left(z_{1}, y_{1}^{\prime}, \ldots, y_{q}^{\prime}\right) \in \mathrm{R}_{\mathbf{b}}^{a}$ such that

$$
x_{1} \wedge z_{1}=0,
$$

and for all $j \in\{1, \ldots, q\}$

$$
y_{j} \wedge y_{j}^{\prime}=n-1 \text {. }
$$

Hence, $y_{j}=y_{j}^{\prime}=n-1$ for all $j \in\{1, \ldots, q\}$. Because of $x_{1} \wedge z_{1}=0$ w.l.o.g. $x_{1}=0$. Thus,

$$
\left(x_{1}, y_{1}, \ldots, y_{q}\right)=(0, n-1, \ldots, n-1) \in \mathrm{R}_{\mathbf{b}}^{a},
$$

which is a contradiction. 
We obtain the second statement, if we apply the duality principle to the first statement.

\subsection{Infinite chains of $C$-clones.}

In contrast to Boolean $C$-clones, we show that for $|D| \geq 3$ there are infinitely many $C$-clones by constructing infinite descending chains of such clones.

Let $\{0,1,2\} \subseteq D$ and $m \in \mathbb{N}_{+}$. Consider the following clausal relation:

$$
\begin{aligned}
& \left.\varrho_{m}:=\mathrm{R}_{(\underbrace{(1, \ldots, 1}_{m \text { times }})}^{\overbrace{1, \ldots, 1}^{m \text { times }}}\right) \\
& \left\{\left(x_{1}, \ldots, x_{m}, y_{1}, \ldots, y_{m}\right) \in D^{2 m} \mid x_{1} \geq 1 \vee \ldots \vee x_{m} \geq 1 \vee y_{1} \leq 1 \vee \ldots \vee y_{m} \leq 1\right\} .
\end{aligned}
$$

Observe that the tuple $(0, \ldots, 0,2, \ldots, 2)$ does not belong to $\varrho_{m}$.

3.5.1 Proposition. If $\varrho_{m}$ is the $2 m$-ary relation defined above, then

$$
\operatorname{Pol}\left(\varrho_{m-1}\right) \supsetneqq \operatorname{Pol}\left(\varrho_{m}\right)
$$

holds for any $m \in \mathbb{N}_{+}$.

Proof. Let $n \in \mathbb{N}_{+}$and $f \in \operatorname{Pol}^{(n)}\left(\varrho_{m}\right)$. We have to show $f \in \operatorname{Pol}^{(n)}\left(\varrho_{m-1}\right)$. Let $r_{1}, \ldots, r_{n} \in \varrho_{m-1}$, where $r_{k}=:\left(x_{1 k}, \ldots, x_{m-1 k}, y_{1 k}, \ldots, y_{m-1 k}\right)$ for $k \in\{1, \ldots, n\}$. Then

$$
\begin{gathered}
x_{1 k} \geq 1 \vee \ldots \vee x_{m-1 k} \geq 1 \vee y_{1 k} \leq 1 \vee \ldots \vee y_{m-1 k} \leq 1 \\
\Leftrightarrow \quad x_{1 k} \geq 1 \vee \ldots \vee x_{m-1 k} \geq 1 \vee x_{m-1 k} \geq 1 \vee y_{1 k} \leq 1 \vee \ldots \vee y_{m-1 k} \leq 1 \vee y_{m-1 k} \leq 1 .
\end{gathered}
$$

We define for $k \in\{1, \ldots, n\}$

$$
\begin{aligned}
r_{k}^{\prime} & =\left(\bar{x}_{1 k}, \ldots, \bar{x}_{m-1 k}, \bar{x}_{m k}, \bar{y}_{1 k}, \ldots, \bar{y}_{m-1 k}, \bar{y}_{m k}\right) \\
& :=\left(x_{1 k}, \ldots, x_{m-1 k}, x_{m-1 k}, y_{1 k}, \ldots, y_{m-1 k}, y_{m-1 k}\right) .
\end{aligned}
$$

The new tuples $r_{1}^{\prime}, \ldots, r_{n}^{\prime}$ belong to $\varrho_{m}$ because of the above expression.

Because $f \triangleright \varrho_{m}$, we have $\left(c_{1}, \ldots, c_{m-1}, c_{m}, d_{1}, \ldots, d_{m-1}, d_{m}\right):=f\left[r_{1}^{\prime}, \ldots, r_{n}^{\prime}\right] \in \varrho_{m}$. Then, by construction, we have $c_{m-1}=c_{m}, d_{m-1}=d_{m}$, and

$$
\begin{aligned}
& c_{m-1} \geq 1 \vee c_{m} \geq 1 \quad \Longleftrightarrow \quad c_{m-1} \geq 1, \\
& d_{m-1} \leq 1 \vee d_{m} \leq 1 \quad \Longleftrightarrow \quad d_{m-1} \leq 1 .
\end{aligned}
$$

Therefore, $\left(c_{1}, \ldots, c_{m-1}, d_{1}, \ldots, d_{m-1}\right)=f\left[r_{1}, \ldots, r_{n}\right] \in \varrho_{m-1}$, i.e. $f \triangleright \varrho_{m-1}$.

Now we show that the inclusion is proper. Let $f$ be a $2 m$-ary operation such that

$$
f\left(x_{1}, \ldots, x_{m}, \ldots, x_{2 m}\right):= \begin{cases}0 & \text { if there is only one } 1 \text { among the first } m \text { entries } \\ & \text { and } 0 \text { in the other entries, } \\ 2 & \text { if there is only one } 1 \text { between the } m+1, \ldots, 2 m \\ \text { entries and } 2 \text { in the other entries, } & \text { otherwise. }\end{cases}
$$


That is

$$
\begin{gathered}
f(1,0, \ldots, \stackrel{m}{0}, 0, \ldots, \stackrel{2 m}{0})=\ldots=f(0, \ldots, 0, \stackrel{m}{1}, 0, \ldots, 0)=0 \\
f(2,2, \ldots, \stackrel{m+1}{1}, 2, \ldots, 2)=\ldots=f(2, \ldots, 2, \stackrel{m+1}{2}, \ldots, 2,1)=2 .
\end{gathered}
$$

We show $f \notin \mathrm{Pol}^{(2 m)}\left(\varrho_{m}\right)$.

Consider the tuples $r_{1}, \ldots r_{2 m}$ belonging to $\varrho_{m}$, such that

$$
r_{1}=(1,0, \ldots, \stackrel{m}{0}, 2, \ldots, \stackrel{2 m}{2}), \ldots, r_{2 m}=(0, \ldots, 0,2, \ldots, 2, \stackrel{2 m}{1}) .
$$

\begin{tabular}{|c|c|c|c|c|c|c|c|c|c|c|c|}
\hline & & 1 & 2 & $\ldots$ & $\mathrm{m}$ & $\mathrm{m}+1$ & $\mathrm{~m}+2$ & $\ldots$ & $2 \mathrm{~m}$ & & \\
\hline 1 & $f($ & 1 & 0 & $\ldots$ & 0 & 0 & 0 & $\ldots$ & 0 & ) & $=0$ \\
\hline 2 & $f($ & 0 & 1 & $\ldots$ & 0 & 0 & 0 & $\ldots$ & 0 & ) & $=0$ \\
\hline $\mathrm{m}$ & $f($ & 0 & 0 & $\ldots$ & 1 & 0 & 0 & $\ldots$ & 0 & ) & $=0$ \\
\hline $\mathrm{m}+1$ & $f($ & 2 & 2 & $\ldots$ & 2 & 1 & 2 & $\ldots$ & 2 & ) & $=2$ \\
\hline $\mathrm{m}+2$ & $f($ & 2 & 2 & $\ldots$ & 2 & 2 & 1 & $\ldots$ & 2 & ) & $=2$ \\
\hline $2 \mathrm{~m}$ & $f($ & 2 & 2 & $\ldots$ & 2 & 2 & 2 & $\ldots$ & 1 & ) & $\begin{array}{c}=2 \\
\notin \varrho_{m}\end{array}$ \\
\hline
\end{tabular}

$f\left[r_{1}, \ldots, r_{2 m}\right] \notin \varrho_{m}$ because,

after we apply $f$ to the tuples in $\varrho_{m}$, we obtain a tuple that does not belong to $\varrho_{m}$. However, $f \in \mathrm{Pol}^{(2 m)}\left(\varrho_{m-1}\right)$ as the following argument shows. Consider any the tuples $r_{1}^{*}, \ldots, r_{2 m}^{*}$ belonging to $\varrho_{m-1}$, where

$$
r_{k}^{*}=\left(x_{1 k}, \ldots, x_{m-1 k}, y_{1 k}, \ldots, y_{m-1 k}\right)
$$

for $k \in\{1, \ldots, 2 m\}$. Note that, by definition, every tuple $r_{k}^{*}$ satisfies the following expression:

$$
x_{1 k} \geq 1 \vee \ldots \vee x_{m-1 k} \geq 1 \vee y_{1 k} \leq 1 \vee \ldots \vee y_{m-1 k} \leq 1 .
$$

Let us regard the tuples $r_{k}^{*} \in \varrho_{m-1}$ as the columns of a matrix $A \in D^{2(m-1) \times(2 m)}$. We construct a matrix $\left(b_{i j}\right)_{\substack{1 \leq i \leq 2(m-1) \\ 1 \leq j \leq 2 m}}=B \in\{0,1\}^{2(m-1) \times(2 m)}$ in the following way:

$$
b_{i j}:= \begin{cases}1 & \text { if } a_{i j} \geq 1 \text { and } 1 \leq i \leq m-1 \\ 1 & \text { if } a_{i j} \leq 1 \text { and } m \leq i \leq 2(m-1) \\ 0 & \text { otherwise }\end{cases}
$$

In every column $r_{k}^{*}$ there is at least one element $d \in D$ such that $d \geq 1$ or $d \leq 1$, hence any column of $B$ contains at least one entry $b_{i j}=1$. Since there are only $2(m-1)$ rows but $2 m$ columns, there is at least one row of $\mathrm{B}$, say the $l$-st row, containing two entries 1. Consequently,

$$
f\left(r_{l 1}^{*}, \ldots, r_{l 2 m}^{*}\right)=1
$$

from the definition of $f$. Then $f\left[r_{1}^{*}, \ldots, r_{2 m}^{*}\right] \in \varrho_{m-1}$, hence $f \triangleright \varrho_{m-1}$. 
Similarly to 3.5 .1 , if we consider

- a fixed number $c$ belonging $\{1, \ldots, n-2\}, m \in \mathbb{N}_{+}$,

- the clausal relation $\varrho_{m}^{\prime}$ defined by

$$
\begin{aligned}
& \left.\varrho_{m}^{\prime}:=\mathrm{R}_{(\underbrace{(c, \ldots, c)}_{m \text { times }}}^{\overbrace{c, \ldots, c}^{m \text { times }}}\right) \\
& =\left\{(\mathbf{x}, \mathbf{y}) \in D^{2 m} \mid x_{1} \geq c \vee \ldots \vee x_{m} \geq c \vee y_{1} \leq c \vee \ldots \vee y_{m} \leq c\right\},
\end{aligned}
$$

- and $g \in \mathrm{O}_{D}^{(2 m)}$ defined by

$$
g\left(x_{1}, \ldots, x_{m}, \ldots, x_{2 m}\right):= \begin{cases}0 & \text { if there is only one } c \text { among the first } \\ n-1 \quad & \text { if entries and } 0 \text { in the other entries } \\ & \text { the entries } m+1, \ldots, 2 m \text { and } n-1 \text { in } c \text { among } \\ & \text { the other entries, } \\ c \quad & \text { otherwise, }\end{cases}
$$

then the proposition below can be proved.

3.5.2 Proposition. Let $c \in\{1, \ldots, n-2\}$ be fixed, and let $\varrho_{m}^{\prime}$ be the $2 m$-ary relation defined above. Then

$$
\operatorname{Pol}\left(\varrho_{m-1}^{\prime}\right) \supsetneqq \operatorname{Pol}\left(\varrho_{m}^{\prime}\right)
$$

holds for any $m \in \mathbb{N}_{+}$.

Next we show that

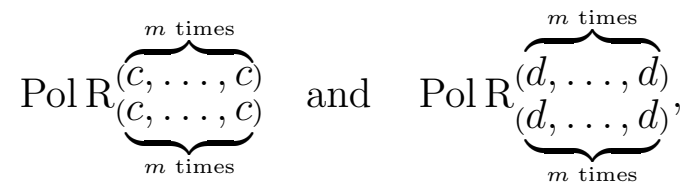

where $m \in \mathbb{N}_{+}, c, d \in D \backslash\{0, n-1\}$ are fixed and $c \neq d$, are incomparable.

Let $m \in \mathbb{N}_{+}$, we define $\sigma_{m}$ by

$\left.\sigma_{m}:=\mathrm{R}_{(\underbrace{(\overbrace{d, \ldots, d}^{m, \ldots, d})}_{m \text { times }}}^{\underbrace{}_{\text {times }}, \ldots}\right)=\left\{(\mathbf{x}, \mathbf{y}) \in D^{2 m} \mid x_{1} \geq d \vee \ldots \vee x_{m} \geq d \vee y_{1} \leq d \vee \ldots \vee y_{m} \leq d\right\}$.

3.5.3 Lemma. Let $c, d \in D \backslash\{0, n-1\}$ be fixed and let $\varrho_{m}^{\prime}$ and $\sigma_{m}$ be the $2 m$-ary relations defined above. If $c \neq d$, then

$$
\operatorname{Pol}\left(\varrho_{m}^{\prime}\right) \nsubseteq \operatorname{Pol}\left(\sigma_{m}\right)
$$

holds for any $m \in \mathbb{N}_{+}$. 
Proof. Fix $c, d \in\{1, \ldots, n-2\}$, consider Pol $\varrho_{m}^{\prime}$ and Pol $\sigma_{m}$, where $c \neq d$. As $c \neq d$, we consider two cases:

1) Let $c<d$ and $f$ be a $2 m$-ary operation such that

$$
f\left(x_{1}, \ldots, x_{m}, \ldots, x_{2 m}\right):= \begin{cases}c & \text { if there is exactly one } d \text { in the first } \\ n-1 & m \text { entries and } 0 \text { in the other entries } \\ n-1 \text { othe. }\end{cases}
$$

It will be shown that $f \triangleright \varrho_{m}^{\prime}$ but $f \not \sigma_{m}$.

Let $\mathbf{r}_{k}:=\left(x_{1 k}, \ldots, x_{m k}, y_{1 k}, \ldots, y_{m k}\right)$ be any tuples, where $k \in\{1, \ldots, 2 m\}$, belonging to $\varrho_{m}^{\prime}$. We define

$$
f\left[\mathbf{r}_{1}, \ldots, \mathbf{r}_{2 m}\right]:=\left(e_{1}, \ldots, e_{m}, o_{1}, \ldots, o_{m}\right) .
$$

As $\operatorname{Im}(f) \in\{c, n-1\}$, we get $f\left(x_{11}, \ldots, x_{12 m}\right)=e_{1} \geq c$, and hence

$$
f\left[\mathbf{r}_{1}, \ldots, \mathbf{r}_{2 m}\right] \in \varrho_{m}^{\prime} .
$$

Thus, $f \triangleright \varrho_{m}^{\prime}$.

Below we show that $f \not \sigma_{m}$. The schema

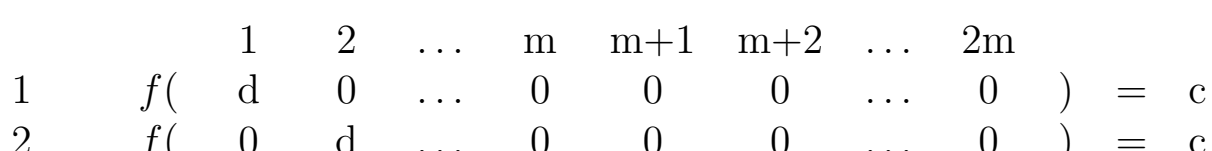

$$
\begin{aligned}
& \left.\begin{array}{llllllllll}
2 & f( & 0 & \mathrm{~d} & \ldots & 0 & 0 & 0 & \ldots & 0
\end{array}\right)=\mathrm{c} \\
& \mathrm{m} \quad f\left(\begin{array}{llllllll}
0 & 0 & \ldots & \mathrm{d} & 0 & 0 & \ldots & 0
\end{array}\right)=\mathrm{c} \\
& \mathrm{m}+1 \quad f\left(\begin{array}{cccccccc}
\mathrm{n}-1 & \mathrm{n}-1 & \ldots & \mathrm{n}-1 & \mathrm{~d} & \mathrm{n}-1 & \ldots & \mathrm{n}-1
\end{array}\right)=\mathrm{n}-1 \\
& \mathrm{~m}+2 \quad f\left(\begin{array}{llllllll}
\mathrm{n}-1 & \mathrm{n}-1 & \ldots & \mathrm{n}-1 & \mathrm{n}-1 & \mathrm{~d} & \ldots & \mathrm{n}-1
\end{array}\right)=\mathrm{n}-1 \\
& 2 \mathrm{~m} \quad f\left(\begin{array}{llllllll}
\mathrm{n}-1 & \mathrm{n}-1 & \ldots & \mathrm{n}-1 & \mathrm{n}-1 & \mathrm{n}-1 & \ldots & \mathrm{d}
\end{array}\right)=\mathrm{n}-1
\end{aligned}
$$

shows that $f \not \sigma_{m}$, because the tuples (columns of the arguments of $f$ ) all belong to $\sigma_{m}$. However, after applying $f$ to the tuples, one obtains a tuple (column) that does not belong to $\sigma_{m}$, (because $c \nsupseteq d$ and $n-1 \not \leq d$ ).

2) If $d<c$, then, similarly to case 1 ) one can prove that Pol $\varrho_{m}^{\prime}$ is not contained in Pol $\sigma_{m}$, by using $g \in \mathrm{O}_{D}^{(2 m)}$ such that

$$
g\left(x_{1}, \ldots, x_{m}, \ldots, x_{2 m}\right):=\left\{\begin{aligned}
c \quad & \text { if there is exactly one } d \text { between the } \\
& m+1, \ldots, 2 m \text { entries and } n-1 \\
& \text { in the other entries, } \\
0 \quad & \text { otherwise. }
\end{aligned}\right.
$$


Note that if $c \neq d$ implies $\mathrm{Pol} \varrho_{m}^{\prime} \nsubseteq \mathbb{\mathrm { Pol }} \sigma_{m}$, then automatically (changing the role of $c$ and $d$, wich remain unequal) $\operatorname{Pol} \sigma_{m} \nsubseteq \mathrm{Pol} \varrho_{m}^{\prime}$ also holds.

From Proposition 3.5.2 and the previous lemma, we get $n-2$ different infinite descending chains of $C$-clones. They are depicted in Figure 3.2 below.

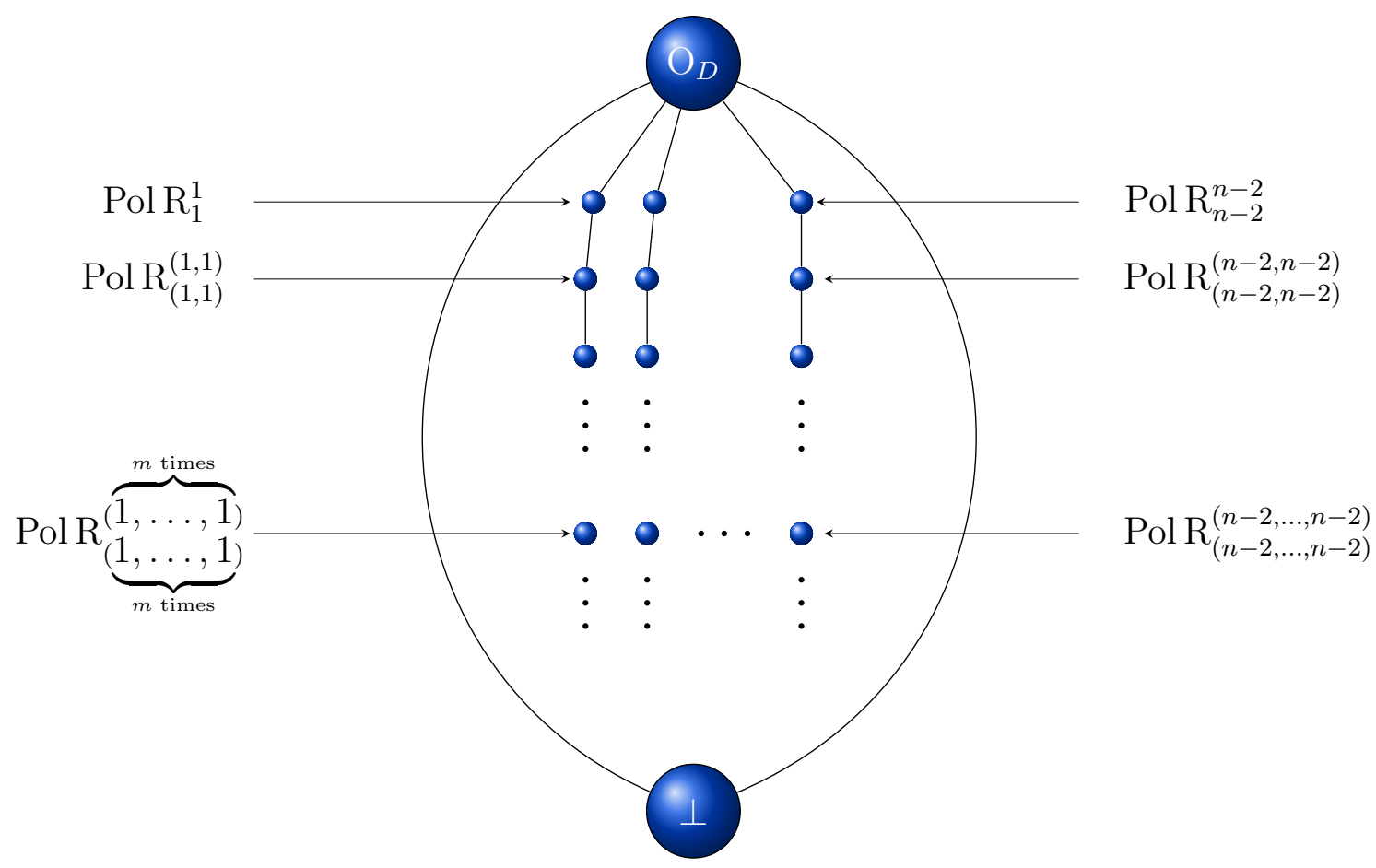

Figure 3.2: Infinite descending chains in the lattice of $C$-clones.

At this point it has been proved that there are infinitely many $C$-clones. However, we cannot answer the following question:

Is the set of all $C$-clones defined on a finite set with at least three elements, countable or uncountable? We leave this question open, which is also presented in Chapter 7.

Below we prove that if we permute the components of $\mathbf{a}$ or $\mathbf{b}$ of the clausal relation $\mathrm{R}_{\mathbf{b}}^{\mathbf{a}}$, the $C$-clone Pol $\mathrm{R}_{\mathbf{b}}^{\mathbf{a}}$ stays the same. To this end we need an auxiliary statement. For a finite set $D$ we have $[Q]_{\mathrm{R}_{D}}=\operatorname{Inv} \operatorname{Pol} Q$, so Pol $[Q]_{\mathrm{R}_{D}}=\operatorname{Pol} \operatorname{Inv} \operatorname{Pol} Q$, whence 3.5.4 follows by setting $Q=\left\{\mathrm{R}_{\mathrm{b}}^{\mathrm{a}}\right\}$.

3.5.4 Remark. Let $\mathrm{R}_{\mathrm{b}}^{\mathrm{a}}$ be a clausal relation. Then, $\mathrm{Pol}\left[\mathrm{R}_{\mathrm{b}}^{\mathrm{a}}\right]_{\mathrm{R}_{D}}=\operatorname{Pol} \mathrm{R}_{\mathrm{b}}^{\mathrm{a}}$ holds.

3.5.5 Lemma. Let $p, q \in \mathbb{N}_{+}, \pi_{1} \in \mathrm{S}_{p}$ and $\pi_{2} \in \mathrm{S}_{q}$. Then

$$
\operatorname{Pol} \mathrm{R}_{\mathbf{b}}^{\mathbf{a}}=\operatorname{Pol} \mathrm{R}_{\mathrm{b} \circ \pi_{2}}^{\mathbf{a} \circ \pi_{1}}
$$

holds, where $\mathbf{a}=\left(a_{1}, \ldots, a_{p}\right) \in(D \backslash\{0\})^{p}, \mathbf{b}=\left(b_{1}, \ldots, b_{q}\right) \in(D \backslash\{n-1\})^{q}$, $\mathbf{a} \circ \pi_{1}=\left(a_{\pi_{1}(1)}, \ldots, a_{\pi_{1}(p)}\right)$, and $\mathbf{b} \circ \pi_{2}=\left(b_{\pi_{2}(1)}, \ldots, b_{\pi_{2}(q)}\right)$. 
Proof. From Lemma 2.2.4, we infer $\mathrm{R}_{\mathbf{b} \circ \pi_{2}}^{\mathrm{a} \circ \pi_{1}}=V_{\pi_{2}}^{\pi_{1}}\left(\mathrm{R}_{\mathbf{b}}^{\mathbf{a}}\right)$. First, we show that the relational clone $\left[\mathrm{R}_{\mathbf{b}}^{\mathbf{a}}\right]_{\mathrm{R}_{D}}$ is equal to $\left[V_{\pi_{2}}^{\pi_{1}}\left(\mathrm{R}_{\mathbf{b}}^{\mathbf{a}}\right)\right]_{\mathrm{R}_{D}}$.

Observe that $V_{\pi_{2}}^{\pi_{1}}\left(\mathrm{R}_{\mathbf{b}}^{\mathbf{a}}\right) \in\left[\mathrm{R}_{\mathbf{b}}^{\mathbf{a}}\right]_{\mathrm{R}_{D}}$. Moreover,

$$
\begin{gathered}
\mathrm{R}_{\mathbf{b}}^{\mathbf{a}}=V_{i d}^{i d}\left(\mathrm{R}_{\mathbf{b}}^{\mathbf{a}}\right)=V_{\pi_{2} \circ \pi_{2}^{-1}}^{\pi_{1} \circ \pi_{1}^{-1}}\left(\mathrm{R}_{\mathbf{b}}^{\mathbf{a}}\right)=V_{\pi_{2}^{-1}}^{\pi_{1}^{-1}}\left(V_{\pi_{2}}^{\pi_{1}}\left(\mathrm{R}_{\mathbf{b}}^{\mathbf{a}}\right)\right) \in\left[V_{\pi_{2}}^{\pi_{1}}\left(\mathrm{R}_{\mathbf{b}}^{\mathbf{a}}\right)\right]_{\mathrm{R}_{D}}, \\
\operatorname{Pol~R}_{\mathbf{b}} \stackrel{3.5 .4}{=} \operatorname{Pol}\left[\mathrm{R}_{\mathbf{b}}^{\mathbf{a}}\right]_{\mathrm{R}_{D}}=\operatorname{Pol}\left[V_{\pi_{2}}^{\pi_{1}}\left(\mathrm{R}_{\mathbf{b}}^{\mathbf{a}}\right)\right]_{\mathrm{R}_{D}}=\operatorname{Pol}\left[\mathrm{R}_{\mathbf{b} \circ \pi_{2}}^{\mathbf{a} \circ \pi_{1}}\right]_{\mathrm{R}_{D}} \stackrel{3.5 .4}{=} \operatorname{Pol~\mathrm {R}_{\mathrm {b}\circ \pi _{2}}^{\mathbf {a}}} .
\end{gathered}
$$

For what is left of this section, we will address the following task.

Given a $C$-clone $\operatorname{Pol}\left(\mathrm{R}_{\mathbf{b}}^{\mathbf{a}, a^{\prime}}\right)$, where

$$
\begin{aligned}
\mathrm{R}_{\mathbf{b}}^{\mathbf{a}, a^{\prime}}= & \left\{\left(x_{1}, \ldots, x_{p}, x_{p+1}, y_{1}, \ldots, y_{q}\right) \in D^{p+q+1} \mid\right. \\
& \left.x_{1} \geq a_{1} \vee \ldots \vee x_{p} \geq a_{p} \vee x_{p+1} \geq a^{\prime} \vee y_{1} \leq b_{1} \vee \ldots \vee y_{q} \leq b_{q}\right\},
\end{aligned}
$$

find a relation $\mathrm{R}_{\mathbf{b}}^{\mathrm{a}}$ of lesser arity, such that

$$
\operatorname{Pol}\left(\mathrm{R}_{\mathbf{b}}^{\mathbf{a}, a^{\prime}}\right) \subseteq \operatorname{Pol}\left(\mathrm{R}_{\mathbf{b}}^{\mathbf{a}}\right) \quad \text { or } \quad \operatorname{Pol}\left(\mathrm{R}_{\mathbf{b}}^{\mathbf{a}}\right) \subseteq \operatorname{Pol}\left(\mathrm{R}_{\mathbf{b}^{\prime}}^{\mathbf{a}, a^{\prime}}\right)
$$

3.5.6 Proposition. Let $p, q \in \mathbb{N}_{+}, \mathbf{a}=\left(a_{1}, \ldots, a_{p}\right) \in(D \backslash\{0\})^{p}, a^{\prime} \in D \backslash\{0\}$ and $\mathbf{b}=\left(b_{1}, \ldots, b_{q}\right) \in(D \backslash\{n-1\})^{q}$. Then

$$
\operatorname{Pol}\left(\mathrm{R}_{\mathbf{b}}^{\mathbf{a}, a^{\prime}}\right) \subseteq \operatorname{Pol}\left(\mathrm{R}_{\mathbf{b}}^{\mathbf{a}}\right)
$$

holds, if and only if there exists some $i \in\{1, \ldots, p\}$, such that $a^{\prime} \geq a_{i}$.

Proof. $\Rightarrow$ Let $k \in \mathbb{N}_{+}$and let $f \in \mathrm{O}_{D}^{(k)}$ be the $k$-ary operation on $D$ defined by

$$
f(\mathbf{x}):= \begin{cases}a^{\prime} & \text { if } \mathbf{x}=\left(a_{1}, \ldots, a_{1}\right) \in(D \backslash\{0\})^{k} \\ n-1 & \text { otherwise }\end{cases}
$$

We show $f \in \operatorname{Pol}^{(k)}\left(\mathrm{R}_{\mathbf{b}}^{\mathbf{a}, a^{\prime}}\right)$. Let $r_{1}, r_{2}, \ldots, r_{k}$ be any tuples belonging to $\mathrm{R}_{\mathbf{b}}^{\mathbf{a}, a^{\prime}}$, where

$$
r_{l}=\left(x_{1 l}, \ldots, x_{p l}, x_{p+1 l}, y_{1 l}, \ldots, y_{q l}\right)
$$

for $l \in\{1, \ldots, k\}$. Then $\left(c_{1}, \ldots, c_{p+1}, d_{1}, \ldots, d_{q}\right):=f\left[r_{1}, \ldots, r_{k}\right] \in \mathrm{R}_{\mathbf{b}}^{\mathbf{a}, a^{\prime}}$ because $c_{p+1}=f\left(x_{p+11}, \ldots, x_{p+1 k}\right) \geq a^{\prime}$. Hence, $f \in \operatorname{Pol}^{(k)}\left(\mathrm{R}_{\mathbf{b}}^{\mathbf{a}, a^{\prime}}\right)$ and $f \in \operatorname{Pol}^{(k)}\left(\mathrm{R}_{\mathbf{b}}^{\mathbf{a}}\right)$ by supposition. Let $r_{1}^{\prime}=\ldots=r_{k}^{\prime}=\left(x_{1}, \ldots, x_{p}, y_{1}, \ldots, y_{q}\right):=\left(a_{1}, \ldots, a_{1}, 0, \ldots, 0\right)$. Obviously, $r_{l}^{\prime}$ belongs to $\mathrm{R}_{\mathbf{b}}^{\mathbf{a}}$ for $l \in\{1, \ldots, k\}$, thus,

$$
f\left[r_{1}^{\prime}, \ldots, r_{k}^{\prime}\right]=\left(a^{\prime}, \ldots, a^{\prime}, n-1, \ldots, n-1\right) \in \mathrm{R}_{\mathbf{b}}^{\mathbf{a}} .
$$

Because $n-1 \not \leq b_{j}$ for all $j \in\{1, \ldots, q\}$, there must be one $i \in\{1, \ldots, p\}$ such that $a^{\prime} \geq a_{i}$. 
$\Leftarrow$ Thanks to 3.5.5, we assume $i=p$, i.e. $a^{\prime} \geq a_{p}$, so $\min \left\{a_{p}, a^{\prime}\right\}=a_{p}$.

Let $f$ be any $k$-ary operation with $f \in \operatorname{Pol}^{(k)}\left(\mathrm{R}_{\mathbf{b}}^{\mathbf{a}, a^{\prime}}\right)$ we have to show $f \in \operatorname{Pol}^{(k)}\left(\mathrm{R}_{\mathbf{b}}^{\mathbf{a}}\right)$. Let $r_{1}, \ldots, r_{k} \in \mathrm{R}_{\mathbf{b}}^{\mathbf{a}}$, where $r_{l}=\left(x_{1 l}, \ldots, x_{p l}, y_{1 l}, \ldots, y_{q l}\right)$ for $l \in\{1, \ldots, k\}$. Then

$$
\begin{gathered}
\quad x_{1 l} \geq a_{1} \vee \ldots \vee x_{p l} \geq a_{p} \vee y_{1 l} \leq b_{1} \vee \ldots \vee y_{q l} \leq b_{q} \\
\Longleftrightarrow \quad x_{1 l} \geq a_{1} \vee \ldots \vee x_{p l} \geq \min \left\{a_{p}, a^{\prime}\right\} \vee y_{1 l} \leq b_{1} \vee \ldots \vee y_{q l} \leq b_{q} \\
\Longleftrightarrow \quad x_{1 l} \geq a_{1} \vee \ldots \vee x_{p l} \geq a_{p} \vee x_{p l} \geq a^{\prime} \vee y_{1 l} \leq b_{1} \vee \ldots \vee y_{q l} \leq b_{q}
\end{gathered}
$$

We define for $l \in\{1, \ldots, k\}$

$$
r_{l}^{\prime}=\left(\bar{x}_{1 l}, \ldots, \bar{x}_{p l}, \bar{x}_{p+1 l}, \bar{y}_{1 l}, \ldots, \bar{y}_{q l}\right):=\left(x_{1 l}, \ldots, x_{p l}, x_{p l}, y_{1 l}, \ldots, y_{q l}\right) .
$$

The tuples $r_{1}^{\prime}, \ldots, r_{k}^{\prime}$ belong to $\mathrm{R}_{\mathbf{b}}^{\mathbf{a}, a^{\prime}}$ because of (3.12).

Because $f \triangleright \mathrm{R}_{\mathbf{b}}^{\mathbf{a}, a^{\prime}}$ we have $\left(c_{1}, \ldots c_{p}, c_{p+1}, d_{1}, \ldots, d_{q}\right):=f\left[r_{1}^{\prime}, \ldots, r_{k}^{\prime}\right] \in \mathrm{R}_{\mathbf{b}}^{\mathbf{a}, a^{\prime}}$. Then, by construction, we have $c_{p}=c_{p+1}$, and

$$
c_{p} \geq a_{p} \vee c_{p+1} \geq a^{\prime} \geq a_{p} \Longleftrightarrow c_{p} \geq a_{p}
$$

Therefore, $\left(c_{1}, \ldots, c_{p}, d_{1}, \ldots, d_{q}\right) \in \mathrm{R}_{\mathbf{b}}^{\mathbf{a}}$, i.e. $f \triangleright \mathrm{R}_{\mathbf{b}}^{\mathbf{a}}$.

Using the duality principle for $C$-clones, the dual statement of 3.5.6 is the following.

3.5.7 Proposition. Let $p, q \in \mathbb{N}_{+}, \mathbf{b}=\left(b_{1}, \ldots, b_{q}\right) \in(D \backslash\{n-1\})^{q}, b^{\prime} \in D \backslash\{n-1\}$ and $\mathbf{a}=\left(a_{1}, \ldots, a_{p}\right) \in(D \backslash\{0\})^{p}$. Then

$$
\operatorname{Pol}\left(\mathrm{R}_{\mathbf{b}, b^{\prime}}^{\mathbf{a}}\right) \subseteq \operatorname{Pol}\left(\mathrm{R}_{\mathbf{b}}^{\mathbf{a}}\right)
$$

holds, if and only if there exists some $j \in\{1, \ldots, q\}$ such that $b^{\prime} \leq b_{j}$.

The following lemma shows a special case where the inclusion, presented in Proposition 3.5.6 above, is proper.

3.5.8 Lemma. Let $k \in \mathbb{N}_{\geq 2}$ and let $|D| \geq 2$. Then

$$
\mathrm{Pol}^{(k)}\left(\mathrm{R}_{0}^{n-1, n-1}\right) \varsubsetneqq \mathrm{Pol}^{(k)}\left(\mathrm{R}_{0}^{n-1}\right) \text {. }
$$

Proof. First, we show $\mathrm{Pol}^{(2)}\left(\mathrm{R}_{0}^{n-1, n-1}\right) \varsubsetneqq \mathrm{Pol}^{(2)}\left(\mathrm{R}_{0}^{n-1}\right)$. To prove the last statement, it is enough, thanks to 3.5.6, to show that $f \triangleright \mathrm{R}_{0}^{n-1}$, but $f \not \mathrm{R}_{0}^{n-1, n-1}$ for some $f \in \mathrm{O}_{D}^{(2)}$. Let $f$ be the binary operation on $D$ defined by

$$
f\left(x_{1}, x_{2}\right):= \begin{cases}n-1 & \text { if } x_{1}=x_{2}=n-1 \\ 0 & \text { otherwise. }\end{cases}
$$


We show that $f$ does not preserve $\mathrm{R}_{0}^{n-1, n-1}$. Consider, $r_{1}=(n-1,0, n-1)$ and $r_{2}=(0, n-1, n-1)$. Clearly, the tuples $r_{1}, r_{2}$ belong to $\mathrm{R}_{0}^{n-1, n-1}$, but $f\left[r_{1}, r_{2}\right]$, which is equal to $(f(n-1,0), f(0, n-1), f(n-1, n-1))=(0,0, n-1)$, does not belong to $\mathrm{R}_{0}^{n-1, n-1}$.

To prove that $f$ preserves $\mathrm{R}_{0}^{n-1}$, we consider any two tuples $r_{1}^{\prime}=\left(x_{1}, y_{1}\right)$ and $r_{2}^{\prime}=\left(x_{2}, y_{2}\right)$ belonging to $\mathrm{R}_{0}^{n-1}$, i.e. $x_{l}=n-1 \vee y_{l}=0$ for $l \in\{1,2\}$. Now we consider two cases:

- If $\left(x_{1}, x_{2}\right)=(n-1, n-1)$, then

$$
f\left[r_{1}^{\prime}, r_{2}^{\prime}\right]=\left(f\left(x_{1}, x_{2}\right), f\left(y_{1}, y_{2}\right)\right)=\left(n-1, f\left(y_{1}, y_{2}\right)\right) \in \mathrm{R}_{0}^{n-1} .
$$

- If $\left(x_{1}, x_{2}\right) \neq(n-1, n-1)$ because $r_{1}^{\prime}, r_{2}^{\prime} \in \mathrm{R}_{0}^{n-1}$, then we get $y_{l}=0$ for some $l \in\{1,2\}$. Hence, $\left(f\left(x_{1}, x_{2}\right), f\left(y_{1}, y_{2}\right)\right)=(0,0) \in \mathrm{R}_{0}^{n-1}$.

To show the lemma for $k \in \mathbb{N}_{\geq 2}$, we define $g\left(x_{1}, x_{2}, \ldots, x_{k}\right)=f\left(x_{1}, x_{2}\right)$. Evidently, $g \in\langle f\rangle_{O_{D}}$, furthermore $f\left(x_{1}, x_{2}\right)=g\left(x_{1}, x_{2}, x_{2} \ldots, x_{2}\right)$, so $f \in\langle g\rangle_{O_{D}}$. Hence, $\langle f\rangle_{O_{D}}=\langle g\rangle_{O_{D}}$, and $\operatorname{Inv} f=\operatorname{Inv}\langle f\rangle_{O_{D}}=\operatorname{Inv}\langle g\rangle_{O_{D}}=\operatorname{Inv} g$. So, because of the first part of the proof, we have $g \triangleright \mathrm{R}_{0}^{n-1}$, but $g \not \mathrm{R}_{0}^{n-1, n-1}$. 



\section{$4 C$-automorphism groups and Krasner $C$-clones}

As we already mentioned our aim is the analysis of the lattice of all $C$-clones. This seems to be fairly hard as we exhibited already infinitely many $C$-clones for $|D| \geq 3$. A first modest step towards our goal is to describe all $C$-clones correlating with Krasner clones on the relational side. Since the latter correspond exactly to all permutation groups on $D$ (cf. [PK79]), there are only finitely many such clones for finite $D$.

\subsection{Characterisation}

In this section we characterise all groups and Krasner clones that are determined by sets of clausal relations. To this end we present a restriction of the Galois connection Aut - Inv, where the relations are clausal relations.

4.1.1 Definition. For $G \subseteq \mathrm{S}_{D}$ we define $C \operatorname{Inv} G:=\operatorname{Inv} G \cap C \mathrm{R}_{D}$. The operators

$$
\begin{aligned}
C \operatorname{Inv}: \mathcal{P}\left(\mathrm{S}_{D}\right) \longrightarrow \mathcal{P}\left(C \mathrm{R}_{D}\right): & & G \mapsto C \operatorname{Inv} G \\
\text { Aut }: \mathcal{P}\left(C \mathrm{R}_{D}\right) \longrightarrow \mathcal{P}\left(\mathrm{S}_{D}\right): & & Q \mapsto \text { Aut } Q
\end{aligned}
$$

define a Galois connection Aut - $C$ Inv between symmetric groups and clausal relations.

4.1.2 Definition. A permutation group $G \subseteq \mathrm{S}_{D}$ is called $C$-automorphism group if $G=$ Aut $Q$ for some set $Q \subseteq C \mathrm{R}_{D}$ of clausal relations, and a set $Q \subseteq C \mathrm{R}_{D}$ is called Krasner $C$-clone if $Q=C \operatorname{Inv} G$ for a permutation group $G$.

In this sense the $C$-automorphism groups allow to analyse the lattice of all $C$-clones up to equality of their unary bijective part. From Remark 3.1.4 we deduce that every $C$-automorphism group can be determined by sets of non-trivial clausal relations, i.e.

4.1.3 Remark. For $Q \subseteq C \mathrm{R}_{D}$, it follows that $\operatorname{Aut}(Q)=\operatorname{Aut}\left(Q \cap C \mathrm{R}_{D}^{*}\right)$.

We shall describe $\left\{\right.$ Aut $\left.Q \mid Q \subseteq C \mathrm{R}_{D}\right\} \stackrel{4.1 .3}{=}\left\{\right.$ Aut $\left.Q^{\prime} \mid Q^{\prime} \subseteq C \mathrm{R}_{D}^{*}\right\}$. Since

$$
\operatorname{Aut} Q^{\prime}=\bigcap_{\mathbf{R}_{\mathbf{b}}^{\mathbf{a}} \in Q^{\prime}} \operatorname{Aut} \mathbf{R}_{\mathbf{b}}^{\mathbf{a}}
$$


for $Q^{\prime} \subseteq C \mathrm{R}_{D}^{*}$, it suffices to regard $C$-automorphism groups ${ }^{1}$ Aut $\mathrm{R}_{\mathbf{b}}^{\mathbf{a}}$ with parameters $\mathbf{a}=\left(a_{1}, \ldots, a_{p}\right) \in(D \backslash\{0\})^{p}$ and $\mathbf{b}=\left(b_{1}, \ldots, b_{q}\right) \in(D \backslash\{n-1\})^{q}$. Observe that, by definition, a clausal relation $\mathrm{R}_{\mathrm{b}}^{\mathbf{a}}$ can be written ${ }^{2}$ as

$$
\mathrm{R}_{\mathbf{b}}^{\mathbf{a}}=\bigcup_{i=1}^{p}\left(D^{i-1} \times\left[a_{i}, n-1\right] \times D^{p+q-i}\right) \cup \bigcup_{j=1}^{q}\left(D^{p+j-1} \times\left[0, b_{j}\right] \times D^{q-j}\right)
$$

For the rest of this section, we are going to study $C$-automorphism groups determined by only one non-trivial clausal relation. In order to characterise them, we use the following lemma, which shows that, on a finite set $D$, permutations preserving some relations automatically preserve its complement.

4.1.4 Lemma ([PK79]). Let $m \in \mathbb{N}_{+}, f \in \mathrm{S}_{D}, \varrho \in \mathrm{R}_{D}^{(m)}$ and let $\varrho^{C}:=D^{m} \backslash \varrho$. Then

$$
f \triangleright \varrho \Longleftrightarrow f^{-1} \triangleright \varrho \Longleftrightarrow f \triangleright \varrho^{C} \Longleftrightarrow f^{-1} \triangleright \varrho^{C} .
$$

Using this lemma, for a permutation $f \in \mathrm{S}_{D}$ and a relation $\mathrm{R}_{\mathbf{b}}^{\mathbf{a}} \in C \mathrm{R}_{D}^{*}$, the proposition $f \triangleright \mathrm{R}_{\mathbf{b}}^{\mathbf{a}}$ can always be replaced by the preservation of the complement $\left(\mathrm{R}_{\mathbf{b}}^{\mathbf{a}}\right)^{C}=\prod_{i=1}^{p}\left[0, a_{i}\right) \times \prod_{j=1}^{q}\left(b_{j}, n-1\right]$. Hence,

$$
\operatorname{Aut} \mathrm{R}_{\mathbf{b}}^{\mathbf{a}}=\operatorname{Aut}\left(\left(\mathrm{R}_{\mathbf{b}}^{\mathbf{a}}\right)^{C}\right)=\operatorname{Aut}\left(\prod_{i=1}^{p}\left[0, a_{i}\right) \times \prod_{j=1}^{q}\left(b_{j}, n-1\right]\right) .
$$

4.1.5 Lemma. Let $\mathrm{R}_{\mathrm{b}}^{\mathrm{a}} \in C \mathrm{R}_{D}^{*}$ be a non-trivial clausal relation, that is to say $\mathbf{a}=\left(a_{1}, \ldots, a_{p}\right) \in(D \backslash\{0\})^{p}$ and $\mathbf{b}=\left(b_{1}, \ldots, b_{q}\right) \in(D \backslash\{n-1\})^{q}$. Then it holds

$$
\begin{aligned}
\operatorname{Pol}\left\{\left(\mathrm{R}_{\mathbf{b}}^{\mathbf{a}}\right)^{C}\right\} & =\operatorname{Pol}\left(\prod_{i=1}^{p}\left[0, a_{i}\right) \times \prod_{j=1}^{q}\left(b_{j}, n-1\right]\right) \\
& =\operatorname{Pol}\left(\left\{\left[0, a_{i}\right) \mid i \in\{1, \ldots, p\}\right\} \cup\left\{\left(b_{j}, n-1\right] \mid j \in\{1, \ldots, q\}\right\}\right) .
\end{aligned}
$$

Proof. Let $W:=\operatorname{Pol}\left(\left\{\left[0, a_{i}\right) \mid i \in\{1, \ldots, p\}\right\} \cup\left\{\left(b_{j}, n-1\right] \mid j \in\{1, \ldots, q\}\right\}\right)$, and let $f \in W$, we show $f \in \operatorname{Pol}\left\{\left(\mathrm{R}_{\mathbf{b}}^{\mathbf{a}}\right)^{C}\right\}$. As $f \in W$, it is equivalent to

$$
\left\{\left[0, a_{i}\right) \mid i \in\{1, \ldots, p\}\right\} \cup\left\{\left(b_{j}, n-1\right] \mid j \in\{1, \ldots, q\}\right\} \subseteq \operatorname{Inv} f .
$$

Since Inv $f$ is a relational clone of $R_{D}$, it is closed under $\times$, it follows that also $\prod_{i=1}^{p}\left[0, a_{i}\right) \times \prod_{j=1}^{q}\left(b_{j}, n-1\right] \in \operatorname{Inv} f$, which is equivalent to

$$
f \in \operatorname{Pol}\left(\prod_{i=1}^{p}\left[0, a_{i}\right) \times \prod_{j=1}^{q}\left(b_{j}, n-1\right]\right)=\operatorname{Pol}\left\{\left(\mathrm{R}_{\mathbf{b}}^{\mathbf{a}}\right)^{C}\right\} .
$$

\footnotetext{
${ }^{1}$ For convenience of notation we occasionally also write Aut $\varrho$ instead of Aut $\{\varrho\}$ for just one relation $\varrho \in \mathrm{R}_{D}$.

${ }^{2}$ Here $[i, j]$ denotes a closed interval of $D$ w.r.t. the natural order on $D$.
} 
Conversely, relational clones are also closed under projections to coordinates, and it holds

$$
\operatorname{pr}_{i}\left(\mathrm{R}_{\mathbf{b}}^{\mathbf{a}}\right)^{C}=\left[0, a_{i}\right) \quad \text { and } \quad \operatorname{pr}_{p+j}\left(\mathrm{R}_{\mathbf{b}}^{\mathbf{a}}\right)^{C}=\left(b_{j}, n-1\right]
$$

for $i \in\{1, \ldots, p\}$ and $j \in\{1, \ldots, q\}$. So if $f \in \operatorname{Pol} \prod_{i=1}^{p}\left[0, a_{i}\right) \times \prod_{j=1}^{q}\left(b_{j}, n-1\right]$, equivalently $\prod_{i=1}^{p}\left[0, a_{i}\right) \times \prod_{j=1}^{q}\left(b_{j}, n-1\right] \in \operatorname{Inv} f$, then also

$$
\operatorname{pr}_{i}\left(\mathrm{R}_{\mathbf{b}}^{\mathbf{a}}\right)^{C}=\left[0, a_{i}\right) \in \operatorname{Inv} f \quad \text { and } \quad \operatorname{pr}_{p+j}\left(\mathrm{R}_{\mathbf{b}}^{\mathbf{a}}\right)^{C}=\left(b_{j}, n-1\right] \in \operatorname{Inv} f
$$

for all $i \in\{1, \ldots, p\}$ and $j \in\{1, \ldots, q\}$. This implies

$$
\left\{\left[0, a_{i}\right) \mid i \in\{1, \ldots, p\}\right\} \cup\left\{\left(b_{j}, n-1\right] \mid j \in\{1, \ldots, q\}\right\} \subseteq \operatorname{Inv} f
$$

equivalently to $f \in \operatorname{Pol}\left(\left\{\left[0, a_{i}\right) \mid i \in\{1, \ldots, p\}\right\} \cup\left\{\left(b_{j}, n-1\right] \mid j \in\{1, \ldots, q\}\right\}\right)$.

By Lemma 4.1.4 it holds for $f \in \mathrm{S}_{D}$ and $b<n-1$

$$
f \triangleright(b, n-1] \Longleftrightarrow f \triangleright(b, n-1]^{C}=[0, b]=[0, b+1),
$$

that is $\operatorname{Aut}(b, n-1]=\operatorname{Aut}[0, b+1)$. Combining this observation with the previous lemma, we obtain for all $\mathrm{R}_{\mathbf{b}}^{\mathbf{a}} \in C \mathrm{R}_{D}^{*}$

$$
\begin{aligned}
\operatorname{Aut}\left\{\mathrm{R}_{\mathbf{b}}^{\mathbf{a}}\right\} & =\operatorname{Aut}\left\{\left(\mathrm{R}_{\mathbf{b}}^{\mathbf{a}}\right)^{C}\right\} \\
& \stackrel{4.1 .5}{=} \operatorname{Aut}\left(\left\{\left[0, a_{i}\right) \mid i \in\{1, \ldots, p\}\right\} \cup\left\{\left(b_{j}, n-1\right] \mid j \in\{1, \ldots, q\}\right\}\right) \\
& =\operatorname{Aut}\left(\left\{\left[0, a_{i}\right) \mid i \in\{1, \ldots, p\}\right\} \cup\left\{\left[0, b_{j}+1\right) \mid j \in\{1, \ldots, q\}\right\}\right) \\
& =\operatorname{Aut}\left(\operatorname{Int} \mathrm{R}_{\mathbf{b}}^{\mathbf{a}}\right),
\end{aligned}
$$

where Int $\mathrm{R}_{\mathrm{b}}^{\mathrm{a}}:=\left\{\left[0, a_{i}\right) \mid i \in\{1, \ldots, p\}\right\} \cup\left\{\left[0, b_{j}+1\right) \mid j \in\{1, \ldots, q\}\right\}$ are the intervals belonging to $\mathrm{R}_{\mathrm{b}}^{\mathrm{a}}$. This enables us to simplify our Galois connection once more:

4.1.6 Corollary ([BV10]). The lattice of $C$-automorphism groups is

$$
\left\{\text { Aut } Q \mid Q \subseteq C \mathrm{R}_{D}\right\}=\{\text { Aut } Q \mid Q \subseteq \mathcal{I}\},
$$

where $\mathcal{I}:=\{[0, a) \mid a \in D \backslash\{0\}\}$.

Proof. For $Q \subseteq C \mathrm{R}_{D}$, Remark 4.1.3 yields

$$
\begin{aligned}
\text { Aut } Q & =\operatorname{Aut}\left(Q \cap C \mathrm{R}_{D}^{*}\right)=\bigcap\left\{\operatorname{Aut}\{\varrho\} \mid \varrho \in Q \cap C \mathrm{R}_{D}^{*}\right\} \\
& =\bigcap\left\{\operatorname{Aut} \operatorname{Int} \varrho \mid \varrho \in Q \cap C \mathrm{R}_{D}^{*}\right\}=\operatorname{Aut}\left(\bigcup\left\{\operatorname{Int} \varrho \mid \varrho \in Q \cap C \mathrm{R}_{D}^{*}\right\}\right),
\end{aligned}
$$

where $\bigcup\left\{\operatorname{Int} \varrho \mid \varrho \in Q \cap C \mathrm{R}_{D}^{*}\right\} \subseteq \mathcal{I}$.

Conversely, for $u \in D \backslash\{0\}$ one has $\operatorname{Int} \mathrm{R}_{(u-1)}^{(u)}=\{[0, u)\}$, and so

$$
\operatorname{Aut}\left\{\mathrm{R}_{(u-1)}^{(u)}\right\}=\operatorname{Aut} \operatorname{Int} \mathrm{R}_{(u-1)}^{(u)}=\operatorname{Aut}\{[0, u)\} .
$$

Hence, for all $U \subseteq D \backslash\{0\}$ holds

$$
\operatorname{Aut}\{[0, u) \mid u \in U\}=\operatorname{Aut}\left\{\mathrm{R}_{(u-1)}^{(u)} \mid u \in U\right\} \text {. }
$$


4.1.7 Corollary. For $Q \subseteq C \mathrm{R}_{D}$ the corresponding Krasner clone is generated by its unary part:

$$
\text { Inv Aut } Q=\operatorname{Inv} \text { Aut Inv }{ }^{(1)} \text { Aut } Q .
$$

Proof. Let $Q^{*}:=Q \cap C \mathrm{R}_{D}^{*}$ denote the non-trivial part of $Q$ and abbreviate

$$
\text { Inv Aut }=:[\cdot]^{\prime} \text {. }
$$

Then as demonstrated in Corollary 4.1.6, one has $\operatorname{Aut}(Q)=\operatorname{Aut}\left(Q^{*}\right)=\operatorname{Aut} Q^{\prime}$, where $Q^{\prime}:=\bigcup\left\{\right.$ Int $\left.\rho \mid \rho \in Q^{*}\right\}$. We will show $Q^{\prime} \subseteq[Q]^{\prime(1)} \subseteq[Q]^{\prime}$, then

$$
[Q]^{\prime}=\operatorname{Inv} \operatorname{Aut}(Q)=\operatorname{Inv} \operatorname{Aut}\left(Q^{\prime}\right)=\left[Q^{\prime}\right]^{\prime} \subseteq\left[[Q]^{\prime(1)}\right]^{\prime} \subseteq\left[[Q]^{\prime}\right]^{\prime}=[Q]^{\prime}
$$

yields the claim. To prove $Q^{\prime} \subseteq[Q]^{\prime(1)}$, it suffices to see Int $\rho \subseteq[Q]^{\prime(1)}$ for any $\rho \in Q^{*} \subseteq Q$. Obviously, Int $\rho$ is a set of unary relations. By definition, for $\rho=\mathrm{R}_{\mathbf{b}}^{\mathbf{a}}$ holds

$$
\left[0, a_{i}\right)=p r_{i}\left(\mathrm{R}_{\mathbf{b}}^{\mathbf{a}}\right)^{C} \in[\rho]^{\prime} \subseteq[Q]^{\prime} \quad \text { and } \quad\left[0, b_{j}+1\right)=\left(p r_{p+j}\left(\mathrm{R}_{\mathbf{b}}^{\mathbf{a}}\right)^{C}\right)^{C} \in[\rho]^{\prime} \subseteq[Q]^{\prime}
$$

for $i \in\{1, \ldots, p\}$ and $j \in\{1, \ldots, q\}$. Thus, Int $\rho \subseteq[Q]^{\prime}$ concluding the argument.

Now the lattice of $C$-automorphism groups can be treated by computer. The following example shows it for $|D|=2,|D|=3$, and $|D|=4$.

4.1.8 Example. In the next cross tables, is shown which $f \in \mathrm{S}_{D}$ preserve which intervals in $\mathcal{I}$. The rows are labelled by functions $f_{k}$ followed by their tuple $z$ of values. Note that the index $k$ corresponds to these tuples as it is just the interpretation of $z=f_{k}(0) f_{k}(1) \ldots f_{k}(n-1)$ as a n-adic number: $k=\sum_{i=0}^{n-1} f_{k}(i)|D|^{n-1-i}$.

\begin{tabular}{|c||c|}
\hline$\triangleright$ & {$[0,1)$} \\
\hline \hline$f_{1}: 01$ & $\times$ \\
\hline$f_{2}: 10$ & \\
\hline
\end{tabular}

\begin{tabular}{|c||c|c|}
\hline$\triangleright$ & {$[0,1)$} & {$[0,2)$} \\
\hline \hline$f_{5}: 012$ & $\times$ & $\times$ \\
\hline$f_{7}: 021$ & $\times$ & \\
\hline$f_{11}: 102$ & & $\times$ \\
\hline$f_{15}: 120$ & & \\
\hline$f_{19}: 201$ & & \\
\hline$f_{21}: 210$ & & \\
\hline
\end{tabular}

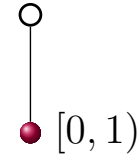

The lattice of all $C$-automorphism groups on the set $D=\{0,1\}$.

$[0,1)$

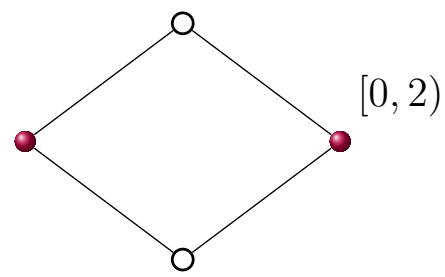

The lattice of all $C$-automorphism groups on the set $D=\{0,1,2\}$. 


\begin{tabular}{|c||c|c|c|}
\hline$\triangleright$ & {$[0,1)$} & {$[0,2)$} & {$[0,3)$} \\
\hline \hline$f_{27}: 0123$ & $\times$ & $\times$ & $\times$ \\
\hline$f_{30}: 0132$ & $\times$ & $\times$ & \\
\hline$f_{39}: 0213$ & $\times$ & & $\times$ \\
\hline$f_{45}: 0231$ & $\times$ & & \\
\hline$f_{54}: 0312$ & $\times$ & & \\
\hline$f_{57}: 0321$ & $\times$ & & \\
\hline$f_{75}: 1023$ & & $\times$ & $\times$ \\
\hline$f_{78}: 1032$ & & $\times$ & \\
\hline$f_{99}: 1203$ & & & $\times$ \\
\hline$f_{108}: 1230$ & & & \\
\hline$f_{114}: 1302$ & & & \\
\hline$f_{120}: 1320$ & & & \\
\hline$f_{135}: 2013$ & & & $\times$ \\
\hline$f_{141}: 2031$ & & & \\
\hline$f_{147}: 2103$ & & & $\times$ \\
\hline$f_{156}: 2130$ & & & \\
\hline$f_{177}: 2301$ & & & \\
\hline$f_{180}: 2310$ & & & \\
\hline$f_{198}: 3012$ & & & \\
\hline$f_{201}: 3021$ & & & \\
\hline$f_{210}: 3102$ & & & \\
\hline$f_{216}: 3120$ & & & \\
\hline$f_{225}: 3201$ & & & \\
\hline$f_{228}: 3210$ & & & \\
\hline
\end{tabular}

$[0,3)$

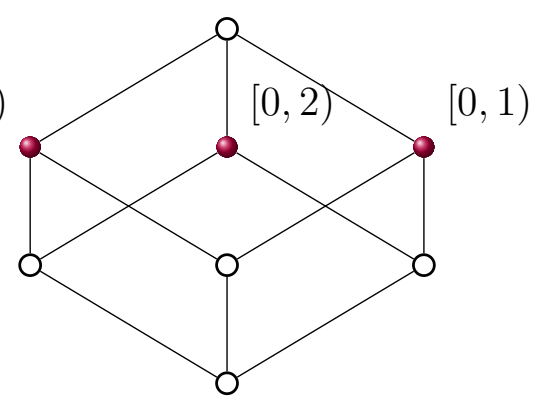

The lattice of all $C$-automorphism groups on the set $D=\{0,1,2,3\}$

In this example it could be seen that the lattice is Boolean with $n-1$ atoms. In fact, this is true in general:

4.1.9 Theorem ([BV10]). The lattice of all $C$-automorphism groups is dually isomorphic to $(\mathcal{P}(D \backslash\{0\}), \subseteq)$ via the following isomorphism

$$
\begin{aligned}
\phi:(\mathcal{P}(D \backslash\{0\}), \subseteq) & \longrightarrow \\
U & \left(\left\{\text { Aut } Q \mid Q \subseteq C \mathrm{R}_{D}\right\}, \supseteq\right) \\
U & \text { Aut }\{[0, u) \mid u \in U\} .
\end{aligned}
$$

Proof. Obviously, $(\mathcal{P}(\mathcal{I}), \subseteq) \cong(\mathcal{P}(D \backslash\{0\}), \subseteq)$, mapping each $U \subseteq D \backslash\{0\}$ to $\{[0, u) \mid u \in U\}$. So from Corollary 4.1.6 one obtains that $\phi$ is a surjective order preserving map. Now it will be shown that it is also order reflecting. This will automatically yield injectivity. To this end we consider $U, U^{\prime} \subseteq D \backslash\{0\}$ such that $\phi(U) \supseteq \phi\left(U^{\prime}\right)$. Take any $u \in U$. Finiteness of $D$ yields that $U^{\prime}=\left\{u_{1}, \ldots, u_{k}\right\}$ such that w.l.o.g. $0=$ : $u_{0}<u_{1}<u_{2}<\ldots<u_{\nu} \leq u<u_{\nu+1}<\ldots<u_{k}$ for some $0 \leq \nu \leq k$, where $1 \leq k \leq|D|$ or $U^{\prime}=\emptyset$. For $U^{\prime} \neq \emptyset$ assume, to obtain 
a contradiction, $u_{\nu}<u$, and let $f \in \mathrm{S}_{D}$ be the transposition swapping $u$ and $u_{\nu}$. Evidently, $f \in \phi\left(U^{\prime}\right) \subseteq \phi(U)$, so $f \triangleright[0, u)$. The assumption $u_{\nu}<u$ yields $u_{\nu} \in[0, u)$, implying $u=f\left(u_{\nu}\right)<u$, a contradiction. Hence, $u=u_{\nu} \in U^{\prime}$, which shows $U \subseteq U^{\prime}$. If $U^{\prime}=\emptyset$ the same argument with $\nu=0$ yields a contradiction as $\phi\left(U^{\prime}\right)=\mathrm{S}_{D}$. Consequently, this case does not occur, concluding the proof.

We finish this section characterising the sort of permutation groups occurring in the lattice of all $C$-automorphism groups. To do this we need an additional auxiliary statement.

4.1.10 Lemma. Let $a_{1}, a_{2} \in D$ with $a_{1}<a_{2}$. Then it holds

$$
\operatorname{Aut}\left\{\left[0, a_{1}\right),\left[a_{1}, a_{2}\right)\right\}=\operatorname{Aut}\left\{\left[0, a_{1}\right),\left[0, a_{2}\right)\right\} .
$$

Proof. The claim is equivalent to the following equality of Krasner clones

$$
\operatorname{Inv} \text { Aut }\left\{\left[0, a_{1}\right),\left[a_{1}, a_{2}\right)\right\}=\operatorname{Inv} \text { Aut }\left\{\left[0, a_{1}\right),\left[0, a_{2}\right)\right\} \text {. }
$$

As Krasner clones are closed under intersection, union and complements, one obtains

$$
\begin{aligned}
{\left[a_{1}, a_{2}\right) } & =\left[0, a_{2}\right) \backslash\left[0, a_{1}\right)=\left[0, a_{2}\right) \cap\left[0, a_{1}\right)^{C} \in \operatorname{Inv} \text { Aut }\left\{\left[0, a_{1}\right),\left[0, a_{2}\right)\right\}, \\
{\left[0, a_{2}\right) } & =\left[0, a_{1}\right) \cup\left[a_{1}, a_{2}\right) \in \operatorname{Inv} \text { Aut }\left\{\left[0, a_{1}\right),\left[a_{1}, a_{2}\right)\right\},
\end{aligned}
$$

showing that the Krasner clones are mutually contained in each other.

4.1.11 Theorem ([BV10]). Let a natural number $m \in \mathbb{N}$ and a finite sequence of elements $0=: a_{0}<a_{1}<\ldots<a_{m}<a_{m+1}:=n$ be given. Then it holds,

$$
\operatorname{Aut}\left\{\left[0, a_{1}\right),\left[0, a_{2}\right), \ldots,\left[0, a_{m}\right)\right\} \cong \mathrm{S}_{\left[a_{0}, a_{1}\right)} \times \mathrm{S}_{\left[a_{1}, a_{2}\right)} \times \ldots \times \mathrm{S}_{\left[a_{m-1}, a_{m}\right)} \times \mathrm{S}_{\left[a_{m}, n\right)} .
$$

Proof. First, we use induction on $m \in \mathbb{N}$ to prove

$$
\operatorname{Aut}\left\{\left[0, a_{1}\right),\left[0, a_{2}\right), \ldots,\left[0, a_{m}\right)\right\}=\operatorname{Aut}\left\{\left[0, a_{1}\right),\left[a_{1}, a_{2}\right), \ldots,\left[a_{m-1}, a_{m}\right)\right\} .
$$

The case $m=0$ is trivial since both sides of the equation yield Aut $\emptyset$. Assuming the claim is true for $m \in \mathbb{N}$, we show its truth for $m+1$ :

$$
\begin{aligned}
\operatorname{Aut}\left\{\left[0, a_{1}\right), \ldots,\left[0, a_{m+1}\right)\right\}= \\
\quad \text { Aut }\left\{\left[0, a_{1}\right), \ldots,\left[0, a_{m-1}\right)\right\} \cap \operatorname{Aut}\left\{\left[0, a_{m}\right),\left[0, a_{m+1}\right)\right\} \\
\stackrel{\text {.1.10 }}{=} \operatorname{Aut}\left\{\left[0, a_{1}\right), \ldots,\left[0, a_{m-1}\right)\right\} \cap \operatorname{Aut}\left\{\left[0, a_{m}\right),\left[a_{m}, a_{m+1}\right)\right\} \\
=\operatorname{Aut}\left\{\left[0, a_{1}\right), \ldots,\left[0, a_{m-1}\right),\left[0, a_{m}\right)\right\} \cap \operatorname{Aut}\left\{\left[a_{m}, a_{m+1}\right)\right\} \\
=\operatorname{Aut}\left\{\left[0, a_{1}\right),\left[a_{1}, a_{2}\right), \ldots,\left[a_{m-1}, a_{m}\right)\right\} \cap \operatorname{Aut}\left\{\left[a_{m}, a_{m+1}\right)\right\} \\
=\operatorname{Aut}\left\{\left[0, a_{1}\right),\left[a_{1}, a_{2}\right), \ldots,\left[a_{m-1}, a_{m}\right),\left[a_{m}, a_{m+1}\right)\right\} .
\end{aligned}
$$

Next we prove

$$
\operatorname{Aut}\left\{\left[a_{0}, a_{1}\right), \ldots,\left[a_{m-1}, a_{m}\right)\right\}=\operatorname{Aut}\left\{\left[a_{0}, a_{1}\right), \ldots,\left[a_{m-1}, a_{m}\right),\left[a_{m}, n\right)\right\} .
$$


Choose $f \in \operatorname{Aut}\left\{\left[a_{0}, a_{1}\right), \ldots,\left[a_{m-1}, a_{m}\right)\right\}=\operatorname{Aut}\left\{\left[0, a_{1}\right),\left[0, a_{2}\right), \ldots,\left[0, a_{m}\right)\right\}$ arbitrarily. Especially, $f \triangleright\left[0, a_{m}\right)$, which is, by Lemma 4.1 .4 , equivalent to $f$ preserves $\left[0, a_{m}\right)^{C}=\left[a_{m}, n\right)$. This shows the inclusion of Aut $\left\{\left[a_{i}, a_{i+1}\right) \mid 0 \leq i<m\right\}$ in Aut $\left\{\left[a_{i}, a_{i+1}\right) \mid 0 \leq i \leq m\right\}$, and the converse is not difficult. Finally, we show that

$$
\operatorname{Aut}\left\{\left[a_{0}, a_{1}\right), \ldots,\left[a_{m-1}, a_{m}\right),\left[a_{m}, n\right)\right\} \cong \mathrm{S}_{\left[a_{0}, a_{1}\right)} \times \ldots \times \mathrm{S}_{\left[a_{m-1}, a_{m}\right)} \times \mathrm{S}_{\left[a_{m}, n\right)} .
$$

We define

$$
\begin{aligned}
\varphi: \mathrm{S}_{\left[a_{0}, a_{1}\right)} \times \ldots \times \mathrm{S}_{\left[a_{m}, n\right)} & \longrightarrow \operatorname{Aut}\left\{\left[a_{0}, a_{1}\right), \ldots,\left[a_{m-1}, a_{m}\right),\left[a_{m}, n\right)\right\} \\
\left(g_{0}, \ldots, g_{m}\right) & \mapsto \varphi\left(g_{0}, \ldots, g_{m}\right)
\end{aligned}
$$

by

$$
\varphi\left(g_{0}, \ldots, g_{m}\right)(x):=g_{i}(x) \text { iff } x \in\left[a_{i}, a_{i+1}\right)
$$

for all $i \in\{0, \ldots, m-1\}$. As the intervals $\left\{\left[a_{i}, a_{i+1}\right) \mid 0 \leq i \leq m\right\}$ form a partition of $D, \varphi\left(g_{0}, \ldots, g_{m}\right)$ is well defined. In order to prove that $\varphi$ is an isomorphism, we have to show that $\varphi$ is a bijective homomorphism. To verify the homomorphism property, take $\left(g_{0}, \ldots, g_{m}\right),\left(h_{0}, \ldots, h_{m}\right) \in \prod_{i=0}^{m} \mathrm{~S}_{\left[a_{i}, a_{i+1}\right)}$ and let $x \in D$. Then, apparently, $x \in\left[a_{i}, a_{i+1}\right)$ for some $0 \leq i \leq m$, and

$$
\begin{aligned}
\varphi\left(g_{0} \circ h_{0}, \ldots, g_{m} \circ h_{m}\right)(x) & =\left(g_{i} \circ h_{i}\right)(x)=g_{i}(x) \circ h_{i}(x) \\
& =\varphi\left(g_{0} \ldots, g_{m}\right)(x) \circ \varphi\left(h_{0}, \ldots, h_{m}\right)(x) .
\end{aligned}
$$

It is clear by definition that $\varphi$ is injective. Finally, we show that $\varphi$ is surjective. Let $h \in \operatorname{Aut}\left\{\left[a_{0}, a_{1}\right), \ldots,\left[a_{m-1}, a_{m}\right),\left[a_{m}, n\right)\right\}$, then we define $g_{i}=\left.h\right|_{\left[a_{i}, a_{i+1}\right)}$, yielding $\varphi\left(g_{1}, \ldots, g_{m}\right)=h$.

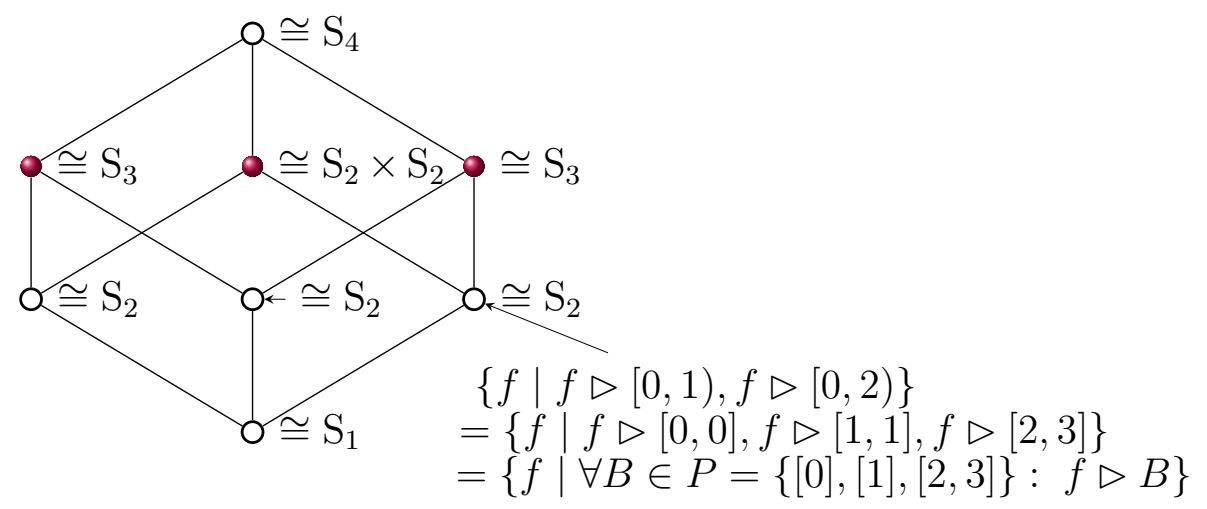

Figure 4.1: The lattice of all $C$-automorphism groups on the set $D=\{0,1,2,3\}$.

Applying the last theorem to all $C$-automorphism groups for $|D|=4$, the case shown in the Example 4.1.8, one obtains the lattice depicted in Figure 4.1. 


\section{2 $C$-automorphism groups and the partition lattice}

The remark in Figure 4.1 hints at another way to understand the structure of this lattice: It is isomorphic (see Proposition 4.2 .1 below) to a sublattice $\mathbb{P}$ of the partition lattice on $D$, consisting of those partitions all blocks of which are intervals w.r.t. the natural order on $D$. That is, $P \in \operatorname{Part}(D)$ is such a partition, iff for every block $B \in P$ holds

$$
[\min B, \max B] \subseteq B .
$$

Combining this observation with the arguments used in the proof of Theorem 4.1.11, one can see that the $C$-automorphism group belonging to such a partition $P$ is given as a direct product of the symmetric groups corresponding to the blocks of $P$ (cf. also Figure 4.1).

4.2.1 Proposition ([BV10]). The lattice of $C$-automorphism groups can be embedded into the lattice $(\operatorname{Part}(D), \leq)$ of all partitions on $D$. The image of this embedding is the lattice $\mathbb{P}:=\{P \in \operatorname{Part}(D) \mid \forall B \in P:[\min B, \max B] \subseteq B\}$.

Proof. By Theorem 4.1.9 it is sufficient to embed $(\mathcal{P}(D \backslash\{0\}), \supseteq)$ in $(\operatorname{Part}(D), \leq)$. We define an order-reversing mapping $\psi: \mathcal{P}(D \backslash\{0\}) \longrightarrow \operatorname{Part}(D)$ in the following way: For every $U \subseteq D \backslash\{0\}$ there is some $m \in \mathbb{N}$ such that $U=\left\{a_{1}, \ldots, a_{m}\right\}$ and $0=: a_{0}<a_{1}<a_{2}<\ldots<a_{m}<a_{m+1}:=n$. Using this we set

$$
\psi(U):=\left\{\left[a_{i}, a_{i+1}\right) \mid 0 \leq i \leq m\right\} .
$$

Clearly, $\psi(U)$ is a partition in $\mathbb{P}$. Conversely, every partition $P \in \mathbb{P}$ arises in the form $P=\psi(U)$ for the set $U:=D \cap\{1+\max B \mid B \in P\}$.

It remains to be shown that $\psi$ is an injective lattice homomorphism. To this end regard $m, k \in \mathbb{N}$ and arbitrary finite sequences

$$
\begin{aligned}
& 0=: a_{0}<a_{1}<a_{2}<\ldots<a_{m}<a_{m+1}:=n \text { and } \\
& 0=: b_{0}<b_{1}<b_{2}<\ldots<b_{k}<b_{k+1}:=n
\end{aligned}
$$

and let $U:=\left\{a_{1}, \ldots, a_{m}\right\}$ and $V:=\left\{b_{1}, \ldots, b_{k}\right\}$.

To show injectivity assume $\psi(U)=\psi(V)$. Now an inductive argument shows $U=V$ : the block $\left[0, a_{1}\right) \in \psi(U)=\psi(V)$, and the only block in $\psi(V)$ containing 0 is $\left[0, b_{1}\right)$, so $\left[0, a_{1}\right)=\left[0, b_{1}\right)$. This implies $a_{1}=b_{1}$. Then

$$
\left[b_{1}, a_{2}\right)=\left[a_{1}, a_{2}\right) \in \psi(U)=\psi(V),
$$

and the only block of $\psi(V)$ containing $b_{1}$ is $\left[b_{1}, b_{2}\right)$. So $\left[a_{1}, a_{2}\right)=\left[b_{1}, b_{2}\right)$, implying $a_{2}=b_{2}$ etc. This continues until $n=a_{m+1}=b_{m+1}$ has been shown, and this means that $b_{m+1}=n=b_{k+1}$. So $k=m$, and $U=V$.

To show $\psi(U \cup V)=\psi(U) \wedge \psi(V)$, take any $v \in U \cup V$ and regard the block $[v, w) \in \psi(U \cup V)$ given by $w:=\min \{y \in U \cup V \mid v<y\}$. Clearly, $a_{i} \leq v<a_{i+1}$ for some $0 \leq i \leq m$, and by definition of $w$, one has $w \leq a_{i+1}$. This shows that the block 
$[v, w) \subseteq\left[a_{i}, a_{i+1}\right) \in \psi(U)$ and, as $v \in U \cup V$ was arbitrary, that $\psi(U \cup V) \leq \psi(U)$. An analogous argument yields $\psi(U \cup V) \leq \psi(V)$, so

$$
\psi(U \cup V) \leq \psi(U) \wedge \psi(V)
$$

Conversely, take any block $B \in \psi(U) \wedge \psi(V)$ and regard some $x \in B$. As

$$
\psi(U) \wedge \psi(V) \leq \psi(U)
$$

there is some $0 \leq i \leq m$ such that $B \subseteq\left[a_{i}, a_{i+1}\right)$. Likewise, there is some $0 \leq j \leq k$ such that $B \subseteq\left[b_{j}, b_{j+1}\right)$. Then also

$$
c:=\max \left\{a_{i}, b_{j}\right\} \leq x<\min \left\{a_{i+1}, b_{j+1}\right\}=: e .
$$

As $B \neq \emptyset, c<e$. Let $d:=\min \{y \in U \cup V \mid c<y\}$, then by minimality $d \leq e$. Assuming $d<e$ and $d \in U$ would contradict $a_{i} \leq c<d<e \leq a_{i+1}$, the assumption on the order in $U$, and similarly, $d \in V$ would contradict $b_{j} \leq c<d<e \leq b_{j+1}$. Hence, $d=e$ and $x \in[c, e)=[c, d)$, i.e. $B \subseteq[c, d) \in \psi(U \cup V)$. As $B \in \psi(U) \wedge \psi(V)$ was arbitrary, this shows $\psi(U) \wedge \psi(V) \leq \psi(U \cup V)$.

Using similar but not necessarily more complicated arguments, below we prove that $\psi(U) \vee \psi(V)=\psi(U \cap V)$.

First we show $\psi(U) \leq \psi(U \cap V)$. Choose any $0 \leq i \leq m$ and define elements

$$
c:=\max \left\{y \in U \cap V \mid y \leq a_{i}\right\} \text { and } d:=\min \{y \in U \cap V \mid c<y\} .
$$

By definition we have $c \leq a_{i}$ and $c<d$. Assuming $d \leq a_{i}$, implies by maximality of $c$ the contradiction $d \leq c<d$. Thus $a_{i}<d$; as $d \in U$ we obtain $a_{i+1} \leq d$. This means $\left[a_{i}, a_{i+1}\right) \subseteq[c, d)$, and as $a_{i} \in U$ was arbitrary, every block in $\psi(U)$ is contained in some block of $\psi(U \cap V)$. This shows

$$
\psi(U) \leq \psi(U \cap V)
$$

and $\psi(V) \leq \psi(U \cap V)$ can be proven with analogous arguments. Hence,

$$
\psi(U) \vee \psi(V) \leq \psi(U \cap V)
$$

To verify $\psi(U) \vee \psi(V) \geq \psi(U \cap V)$, we regard any block $[u, v) \in \psi(U \cap V)$. Note that $v=\min \{y \in U \mid y>u$ and $y \in V\}$. As $u, v \in U$, there is a subsequence

$$
u=a_{0}^{\prime}<a_{1}^{\prime}<a_{2}^{\prime}<\cdots<a_{t}^{\prime}=v
$$

of $a_{0}, a_{1}, a_{2}, \ldots, a_{m}$, such that $[u, v)=\bigcup_{0 \leq s<t}\left[a_{s}^{\prime}, a_{s+1}^{\prime}\right)$.

We show by induction on $1 \leq r \leq t$ that there is some block $B \in \psi(U) \vee \psi(V)$ with $\left[a_{0}^{\prime}, a_{r}^{\prime}\right) \subseteq B$. The base $r=1$ is simple: $\left[a_{0}^{\prime}, a_{1}^{\prime}\right) \in \psi(U) \leq \psi(U) \vee \psi(V)$, so there is such a block $B$. We now proceed with the inductive step from $1 \leq r$ to $r+1 \leq t$. By the induction hypothesis, we know of the existence of a block $B \in \psi(U) \vee \psi(V)$ with $\left[a_{0}^{\prime}, a_{r}^{\prime}\right) \subseteq B$. Let $b:=\max \left\{y \in V \mid y \leq a_{r}^{\prime}\right\}$, especially $b \leq a_{r}^{\prime}$. Since $0<r<t$, 
we have $u=a_{0}^{\prime}<a_{r}^{\prime}<a_{t}^{\prime}=v$, so by minimality of $v, a_{r}^{\prime} \notin V$. This shows $a_{r}^{\prime} \neq b$, i.e. $a_{r}^{\prime}<b$. Define $b^{+}:=\min \{y \in V \mid y>b\}$, then $b<b^{+}$. The maximality of $b$ implies $b^{+}>a_{r}^{\prime}$, i.e. $b<a_{r}^{\prime}<b^{+}$. Thus, $\left[b, b^{+}\right) \in \psi(V) \leq \psi(U) \vee \psi(V)$. Hence, there is some block $B^{\prime} \in \psi(U) \vee \psi(V)$ with $a_{r}^{\prime} \in\left[b, b^{+}\right) \subseteq B^{\prime}$. Furthermore, as $V \ni u=a_{0}^{\prime}<a_{r}^{\prime}$, the maximality of $b$ implies $u=a_{0}^{\prime} \leq b<a_{r}^{\prime}$, so $b \in\left[a_{0}^{\prime}, a_{r}^{\prime}\right) \subseteq B$. Thus $b \in B \cap B^{\prime}$, i.e. $B=B^{\prime}$ as they are blocks of a partition. Putting this together with our previous observations, we obtain $a_{r}^{\prime} \in\left[b, b^{+}\right) \subseteq B^{\prime}=B$. Since $\left[a_{r}^{\prime}, a_{r+1}^{\prime}\right) \in \psi(U) \leq \psi(U) \vee \psi(V)$, there is a block $B^{\prime \prime} \in \psi(U) \vee \psi(V)$ such that $a_{r}^{\prime} \in\left[a_{r}^{\prime}, a_{r+1}^{\prime}\right) \subseteq B^{\prime \prime}$. Hence $a_{r}^{\prime} \in B \cap B^{\prime \prime}$, so $B=B^{\prime \prime}$ and $\left[a_{r}^{\prime}, a_{r+1}^{\prime}\right) \subseteq B^{\prime \prime}=B$. This shows

$$
\left[a_{0}^{\prime}, a_{r+1}^{\prime}\right)=\left[a_{0}^{\prime}, a_{r}^{\prime}\right) \cup\left[a_{r}^{\prime}, a_{r+1}^{\prime}\right) \subseteq B,
$$

finishing the induction.

Using the result just proven for $r=t$, one obtains $[u, v]=\left[a_{0}^{\prime}, a_{t}^{\prime}\right) \in B$ for some block $B \in \psi(U) \vee \psi(V)$. For the block $[u, v) \in \psi(U \cap V)$ was arbitrarily chosen, this yields $\psi(U \cap V) \leq \psi(U) \vee \psi(V)$, concluding the proof. 


\section{$5 C$-monoids and weak Krasner $C$-clones}

In this chapter we study the $C$-clone lattice on a finite set up to equality of the corresponding endomorphism monoids. Here, unary relations are not any more enough to determine the lattice of all relational clones given by $C$-monoids, as given by $C$-automorphism groups (See Chapter 4 ), and hence its structure is considerably harder to understand.

\subsection{Describing $C$-monoids}

In this section we describe some monoids and weak Krasner clones that are determined by sets of clausal relations. The restriction of the Galois connection End - Inv where the relations are clausal relations define the following Galois connection.

5.1.1 Definition. For $F \subseteq \mathrm{O}_{D}^{(1)}$ we define $C \operatorname{Inv} F:=\operatorname{Inv} F \cap C \mathrm{R}_{D}$. The operators

$$
\begin{array}{rlrl}
C \text { Inv }: \mathcal{P}\left(\mathrm{O}_{D}^{(1)}\right) \longrightarrow \mathcal{P}\left(C \mathrm{R}_{D}\right): & & F \mapsto C \operatorname{Inv} F \\
\text { End }: \mathcal{P}\left(C \mathrm{R}_{D}\right) \longrightarrow \mathcal{P}\left(\mathrm{O}_{D}^{(1)}\right): & Q \mapsto \operatorname{End} Q
\end{array}
$$

define a Galois connection End - $C$ Inv between unary operations and clausal relations.

5.1.2 Definition. A set $F \subseteq \mathrm{O}_{D}^{(1)}$ is called a clausal monoid, or $C$-monoid for short if $F=$ End $Q$ for some set $Q \subseteq C \mathrm{R}_{D}$ of clausal relations. A set $Q \subseteq C \mathrm{R}_{D}$ is called a weak Krasner $C$-clone if $Q=C \operatorname{Inv} F$ for some set $F$ of unary operations.

Every Galois connection gives rise to a pair of closure operators. For the ones belonging to Definition 5.1.1 we introduce a special notation.

5.1.3 Definition. For any $F \subseteq \mathrm{O}_{D}^{(1)}$ and any $Q \subseteq C \mathrm{R}_{D}$ we set

$$
\begin{aligned}
\langle F\rangle_{\mathcal{C O}}^{(1)} & :=\operatorname{End} C \operatorname{Inv} F, \\
{[Q]_{w \mathcal{C}} } & :=C \operatorname{Inv} \text { End } Q .
\end{aligned}
$$

Our aim is to describe $\left\{\right.$ End $\left.Q \mid Q \subseteq C \mathrm{R}_{D}\right\}$ all $C$-monoids on $D$. According to Remark 3.1.4 every $C$-monoid can be determined by sets of non-trivial clausal relations, i.e.

$$
\left\{\text { End } Q \mid Q \subseteq C \mathrm{R}_{D}\right\} \stackrel{3.1 .4}{=}\left\{\text { End } Q^{\prime} \mid Q^{\prime} \subseteq C \mathrm{R}_{D}^{*}\right\}
$$


Since

$$
\text { End } Q^{\prime}=\bigcap_{\mathrm{R}_{\mathbf{b}}^{\mathbf{a}} \in Q^{\prime}} \text { End } \mathbf{R}_{\mathbf{b}}^{\mathbf{a}}
$$

for $Q^{\prime} \subseteq C \mathrm{R}_{D}^{*}$, it suffices to regard $C$-monoids of the form End $\mathrm{R}_{\mathbf{b}}^{\mathbf{a}}$ with parameters $\mathbf{a}=\left(a_{1}, \ldots, a_{p}\right) \in(D \backslash\{0\})^{p}$ and $\mathbf{b}=\left(b_{1}, \ldots, b_{q}\right) \in(D \backslash\{n-1\})^{q}$.

With the help of Propositions 5.1.4, 5.1.5, and Corollary 5.1.6 below we can identify finite subsets of clausal relations, which are sufficient to describe the lattice of all $C$-monoids.

5.1.4 Proposition. Let $\mathbf{a}=\left(a_{1}, \ldots, a_{p}\right) \in(D \backslash\{0\})^{p}$ and $b_{1}, \ldots, b_{q} \in D \backslash\{n-1\}$, where $p, q \in \mathbb{N}_{+}$. Then, it follows that

$$
\text { End } \mathrm{R}_{b_{1}, \ldots, b_{q}, b_{q}}^{\mathrm{a}}=\text { End } \mathrm{R}_{b_{1}, \ldots, b_{q}}^{\mathrm{a}} \text {. }
$$

Proof. " $\subseteq$ ": Let $f \in$ End $\mathrm{R}_{b_{1}, \ldots, b_{q}, b_{q}}^{\mathrm{a}}$. We have to show $f \in \operatorname{End~}_{b_{1}, \ldots, b_{q}}^{\mathrm{a}}$. Let $\mathbf{r}:=\left(x_{1}, \ldots, x_{p}, y_{1}, \ldots, y_{q}\right) \in \mathrm{R}_{b_{1}, \ldots, b_{q}}^{\mathbf{a}}$, i.e.

$$
\begin{aligned}
& x_{1} \geq a_{1} \vee \ldots \vee x_{p} \geq a_{p} \vee y_{1} \leq b_{1} \vee \ldots \vee y_{q} \leq b_{q} \\
\Longleftrightarrow \quad & x_{1} \geq a_{1} \vee \ldots \vee x_{p} \geq a_{p} \vee y_{1} \leq b_{1} \vee \ldots \vee y_{q} \leq b_{q} \vee y_{q} \leq b_{q} .
\end{aligned}
$$

Because of the above expression we obtain $\left(x_{1}, \ldots, x_{p}, y_{1}, \ldots, y_{q}, y_{q}\right) \in \mathrm{R}_{b_{1}, \ldots, b_{q}, b_{q}}^{\mathbf{a}}$. Since $f \triangleright \mathrm{R}_{b_{1}, \ldots, b_{q}, b_{q}}^{\mathrm{a}}$, we have

$$
\begin{aligned}
& f\left(x_{1}\right) \geq a_{1} \vee \ldots \vee f\left(x_{p}\right) \geq a_{p} \vee f\left(y_{1}\right) \leq b_{1} \vee \ldots \vee f\left(y_{q}\right) \leq b_{q} \vee f\left(y_{q}\right) \leq b_{q} \\
\Longleftrightarrow & f\left(x_{1}\right) \geq a_{1} \vee \ldots \vee f\left(x_{p}\right) \geq a_{p} \vee f\left(y_{1}\right) \leq b_{1} \vee \ldots \vee f\left(y_{q}\right) \leq b_{q} \\
\Longleftrightarrow & f[\mathbf{r}] \in \mathrm{R}_{b_{1}, \ldots, b_{q}}^{\mathbf{a}} .
\end{aligned}
$$

" $\supseteq$ ": Let $f \in$ End $\mathrm{R}_{b_{1}, \ldots, b_{q}}^{\mathbf{a}}$. Assume that $f \notin$ End $\mathrm{R}_{b_{1}, \ldots, b_{q}, b_{q}}^{\mathrm{a}}$, hence there is one $\mathbf{r}^{\prime}:=\left(x_{1}^{\prime}, \ldots, x_{p}^{\prime}, y_{1}^{\prime}, \ldots, y_{q}^{\prime}, y_{q+1}^{\prime}\right) \in \mathrm{R}_{b_{1}, \ldots, b_{q}, b_{q}}^{\mathbf{a}}$ such that $f\left[\mathbf{r}^{\prime}\right] \notin \mathrm{R}_{b_{1}, \ldots, b_{q}, b_{q}}^{\mathrm{a}}$, i.e.

$$
x_{1}^{\prime} \geq a_{1} \vee \ldots \vee x_{p}^{\prime} \geq a_{p} \vee y_{1}^{\prime} \leq b_{1} \vee \ldots \vee y_{q}^{\prime} \leq b_{q} \vee y_{q+1}^{\prime} \leq b_{q}
$$

and $f\left(x_{1}^{\prime}\right)<a_{1} \wedge \ldots \wedge f\left(x_{p}^{\prime}\right)<a_{p} \wedge f\left(y_{1}^{\prime}\right)>b_{1} \wedge \ldots \wedge f\left(y_{q}^{\prime}\right)>b_{q} \wedge f\left(y_{q+1}^{\prime}\right)>b_{q}$.

We consider two cases:

Case 1: If $\mathbf{r}^{*}:=\left(x_{1}^{\prime}, \ldots, x_{p}^{\prime}, y_{1}^{\prime}, \ldots, y_{q}^{\prime}\right) \in \mathrm{R}_{b_{1}, \ldots, b_{q}}^{\mathbf{a}}$, then it follows that $f\left(x_{i}^{\prime}\right)<a_{i}$ for all $i$ belonging to $\{1, \ldots, p\}$ and $f\left(y_{j}^{\prime}\right)>b_{j}$ for all $j \in\{1, \ldots, q\}$. Consequently, $f\left[\mathbf{r}^{*}\right]$ does not belong to $\mathrm{R}_{b_{1}, \ldots, b_{q}}^{\mathbf{a}}$, which is a contradiction to $f \triangleright \mathrm{R}_{b_{1}, \ldots, b_{q}}^{\mathbf{a}}$.

Case 2: If $\mathbf{r}^{*} \notin \mathrm{R}_{b_{1}, \ldots, b_{q}}^{\mathrm{a}}$, i.e.,

$$
x_{1}^{\prime}<a_{1} \wedge \ldots \wedge x_{p}^{\prime}<a_{p} \wedge y_{1}^{\prime}>b_{1} \wedge \ldots \wedge y_{q}^{\prime}>b_{q} .
$$

Because of (5.1) we have $y_{q+1}^{\prime} \leq b_{q}$. Furthermore, the tuple

$$
\mathbf{r}^{* *}:=\left(x_{1}^{\prime}, \ldots, x_{p}^{\prime}, y_{1}^{\prime}, \ldots, y_{q-1}^{\prime}, y_{q+1}^{\prime}\right)
$$

belongs to $\mathrm{R}_{b_{1}, \ldots, b_{q}}^{\mathrm{a}}$. However,

$$
f\left(x_{1}^{\prime}\right)<a_{1} \wedge \ldots \wedge f\left(x_{p}^{\prime}\right)<a_{p} \wedge f\left(y_{1}^{\prime}\right)>b_{1} \wedge \ldots \wedge f\left(y_{q-1}^{\prime}\right)>b_{q-1} \wedge f\left(y_{q+1}^{\prime}\right)>b_{q} .
$$

Thus, we get $f\left[\mathbf{r}^{* *}\right] \notin \mathrm{R}_{b_{1}, \ldots, b_{q}}^{\mathbf{a}}$, which is also a contradiction to $f \triangleright \mathrm{R}_{b_{1}, \ldots, b_{q}}^{\mathbf{a}}$. 
The dual statement of 5.1.4 is the following.

5.1.5 Proposition. Let $a_{1}, \ldots, a_{p} \in D \backslash\{0\}$ and $\mathbf{b}=\left(b_{1}, \ldots, b_{q}\right) \in(D \backslash\{n-1\})^{q}$, where $p, q \in \mathbb{N}_{+}$. Then it follows that

$$
\text { End } \mathrm{R}_{\mathbf{b}}^{a_{1}, \ldots, a_{p}, a_{p}}=\text { End } \mathrm{R}_{\mathbf{b}}^{a_{1}, \ldots, a_{p}} .
$$

From Lemma 3.5.5 we infer that if we permute the components of $\mathbf{a}$ or $\mathbf{b}$ of the clausal relation $\mathrm{R}_{\mathrm{b}}^{\mathrm{a}}$, the $C$-monoid End $\mathrm{R}_{\mathrm{b}}^{\mathrm{a}}$ stays the same. This is pointed out in the following corollary.

5.1.6 Corollary. Let $p, q \in \mathbb{N}_{+}, \pi_{1} \in \mathrm{S}_{p}$ and $\pi_{2} \in \mathrm{S}_{q}$. Then

$$
\text { End } \mathrm{R}_{\mathbf{b}}^{\mathbf{a}}=\text { End } \mathrm{R}_{\mathbf{b} \circ \pi_{2}}^{\mathbf{a} \circ \pi_{1}}
$$

holds, where $\mathbf{a}=\left(a_{1}, \ldots, a_{p}\right) \in(D \backslash\{0\})^{p}, \mathbf{b}=\left(b_{1}, \ldots, b_{q}\right) \in(D \backslash\{n-1\})^{q}$, $\mathbf{a} \circ \pi_{1}=\left(a_{\pi_{1}(1)}, \ldots, a_{\pi_{1}(p)}\right)$, and $\mathbf{b} \circ \pi_{2}=\left(b_{\pi_{2}(1)}, \ldots, b_{\pi_{2}(q)}\right)$.

Due to Propositions 5.1.4, 5.1.5 and Corollary 5.1.6, it is sufficient for the study of $C$-monoids to regard $C$-monoids End $\mathrm{R}_{\mathbf{b}}^{\mathbf{a}}$, where $\mathrm{R}_{\mathbf{b}}^{\mathbf{a}} \in C \mathrm{R}_{D}^{*}$ is a non-trivial clausal relation, and all entries of the tuple $\mathbf{a}$ and of the tuple $\mathbf{b}$ are pairwise distinct, respectively. This implies that $1 \leq q, p<n$.

Reordering the entries in the tuples of a relation does not change its set of polymorphisms. This, implies that reordering the components of $\mathbf{a}$ or $\mathbf{b}$ of a clausal relation $R_{b}^{a}$, does not change the set of endomorphisms that preserve it. That is End $R_{\mathbf{b}}^{\mathbf{a}}=$ End $\mathrm{R}_{\widetilde{\mathbf{b}}}^{\widetilde{\mathbf{a}}}$, where $\widetilde{\mathbf{a}}$ has the same entries as $\mathbf{a}$ but in ascending order and $\widetilde{\mathbf{b}}$ has the same entries as $\mathbf{b}$ but in descending order. In the following sections we will only consider such clausal relations.

From Propositions 3.5.6 and 3.5.7 we infer the following corollaries.

5.1.7 Corollary. Let $p, q \in\{1, \ldots, n-1\}, \mathbf{b}=\left(b_{1}, \ldots, b_{q}\right) \in(D \backslash\{n-1\})^{q}$, and $a_{1}, \ldots, a_{p}, a^{\prime} \in D \backslash\{0\}$. Then the inclusion

$$
\text { End } \mathrm{R}_{\mathbf{b}}^{a_{1}, \ldots, a_{p}, a^{\prime}} \subseteq \text { End } \mathrm{R}_{\mathbf{b}}^{a_{1}, \ldots, a_{p}}
$$

holds if and only if there exists some $i \in\{1, \ldots, p\}$ with $a^{\prime} \geq a_{i}$.

5.1.8 Corollary. Let $p, q \in\{1, \ldots, n-1\}, \mathbf{a}=\left(a_{1}, \ldots, a_{p}\right) \in(D \backslash\{0\})^{p}$, and $b_{1}, \ldots, b_{q}, b^{\prime} \in D \backslash\{n-1\}$. Then the inclusion

$$
\text { End } \mathrm{R}_{b_{1}, \ldots, b_{q}, b^{\prime}}^{\mathbf{a}} \subseteq \text { End } \mathrm{R}_{b_{1}, \ldots, b_{q}}^{\mathbf{a}}
$$

holds if and only if there exists some $j \in\{1, \ldots, q\}$ with $b^{\prime} \leq b_{j}$.

From Propositions and Corollaries 5.1.4 to 5.1.8 we infer the existence of the following finite descending chains in the lattice of $C$-monoids on a finite set $D$ :

$$
\text { End } \mathrm{R}_{0}^{1,2, \ldots, n-2, n-1} \subseteq \text { End } \mathrm{R}_{0}^{1,2, \ldots, n-2} \subseteq \ldots \subseteq \text { End } \mathrm{R}_{0}^{1,2} \subseteq \text { End } \mathrm{R}_{0}^{1}
$$


and

End $\mathrm{R}_{n-2, n-3, \ldots, 1,0}^{n-1} \subseteq$ End $\mathrm{R}_{n-2, n-3, \ldots, 1}^{n-1} \subseteq \ldots \subseteq$ End $\mathrm{R}_{n-2, n-3}^{n-1} \subseteq$ End $\mathrm{R}_{n-2}^{n-1}$.

Lemma 5.1.9 shows some equalities between $C$-monoids, which enable us to reduce the number of non-trivial clausal relations to consider for determining $C$-monoids. Corollary 5.1.10 below states the numbers of relations resulting from this reduction.

5.1.9 Lemma. Let $f \in \mathrm{O}_{D}^{(1)}, \mathbf{a} \in(D \backslash\{0\})^{p}, \mathbf{b} \in(D \backslash\{n-1\})^{q}$. Then we have:

1. For $p \geq 0$ and $q \geq 1$ the following equivalence holds:

$$
f \triangleright \mathrm{R}_{\mathbf{b}}^{1, \mathbf{a}} \Longleftrightarrow f \triangleright \mathrm{R}_{\mathbf{b}, 0}^{1, \mathbf{a}} .
$$

Consequently, End $\mathrm{R}_{\mathbf{b}}^{1, \mathbf{a}}=$ End $\mathrm{R}_{\mathbf{b}, 0}^{1, \mathbf{a}}$.

2. For $p \geq 1$ and $q \geq 0$ the following equivalence holds:

$$
f \triangleright \mathrm{R}_{n-2, \mathbf{b}}^{\mathbf{a}} \Longleftrightarrow f \triangleright \mathrm{R}_{n-2, \mathbf{b}}^{\mathbf{a}, n-1}
$$

Consequently, End $\mathrm{R}_{n-2, \mathbf{b}}^{\mathbf{a}}=$ End $\mathrm{R}_{n-2, \mathbf{b}}^{\mathbf{a}, n-1}$.

Proof. We show that End $\mathrm{R}_{\mathbf{b}}^{1, \mathbf{a}}=$ End $\mathrm{R}_{\mathbf{b}, 0}^{1, \mathbf{a}}$. The inclusion " $\supseteq$ " follows from Corollary 5.1.8. Conversely, let $f \in \operatorname{End~}_{\mathbf{b}}^{1, \mathbf{a}}$, and let $\mathbf{r}:=(u, \mathbf{x}, \mathbf{y}, v) \in \mathrm{R}_{\mathbf{b}, 0}^{1, \mathbf{a}}$. The latter is equivalent to $(u, \mathbf{x}, \mathbf{y}) \in \mathrm{R}_{\mathbf{b}}^{1, \mathbf{a}}$ or $v=0$. We consider two cases:

- If $(u, \mathbf{x}, \mathbf{y}) \in \mathrm{R}_{\mathbf{b}}^{1, \mathbf{a}}$, then from $f \triangleright \mathrm{R}_{\mathbf{b}}^{1, \mathbf{a}}$ we obtain that $(f(u), f[\mathbf{x}], f[\mathbf{y}]) \in \mathbf{R}_{\mathbf{b}}^{1, \mathbf{a}}$. Hence, we get that $f[\mathbf{r}]=(f(u), f[\mathbf{x}], f[\mathbf{y}], f(v)) \in \mathrm{R}_{\mathbf{b}, 0}^{1, \mathbf{a}}$.

- If $(u, \mathbf{x}, \mathbf{y}) \notin \mathrm{R}_{\mathbf{b}}^{1, \mathbf{a}}$, then we have $u=0$ and $(\mathbf{x}, \mathbf{y}) \notin \mathrm{R}_{\mathbf{b}}^{\mathbf{a}}$. Since $\mathbf{r} \in \mathrm{R}_{\mathbf{b}, 0}^{1, \mathbf{a}}$, we obtain $v=0$. Let us assume $f[\mathbf{r}]=(f(u), f[\mathbf{x}], f[\mathbf{y}], f(v)) \notin \mathrm{R}_{\mathbf{b}, 0}^{1, \mathbf{a}}$. Hence, $0=f(u)$ and $0=f(u)=f(0)=f(v)>0$, which is a contradiction. Thus, it follows that $f[\mathbf{r}] \in \mathrm{R}_{\mathbf{b}, 0}^{1, \mathbf{a}}$.

The second statement follows from the duality principle for $C$-clones:

$$
\begin{aligned}
& f \triangleright \mathrm{R}_{n-2, \mathbf{b}}^{\mathbf{a}} \stackrel{3.3 .4}{\Longleftrightarrow} f^{\alpha} \triangleright \mathrm{R}_{\mathbf{a}^{\alpha}}^{n-2^{\alpha}, \mathbf{b}^{\alpha}} \Longleftrightarrow f^{\alpha} \triangleright \mathrm{R}_{n-1-\mathbf{a}}^{1, n-1-\mathbf{b}} \\
& \stackrel{\text { first part }}{\Longleftrightarrow} f^{\alpha} \triangleright \mathrm{R}_{n-1-\mathbf{a}, 0}^{1, n-1-\mathbf{b}} \stackrel{3.3 .4}{\Longleftrightarrow} f=\left(f^{\alpha}\right)^{\alpha} \triangleright \mathrm{R}_{1^{\alpha},(n-1-\mathbf{b})^{\alpha}}^{(n-1-\mathbf{a})^{\alpha}, 0^{\alpha}}=\mathrm{R}_{n-2, \mathbf{b}}^{\mathbf{a}, n-1} .
\end{aligned}
$$

Applying Lemma 5.1.9 to $C$-monoids on $D=\{0,1,2\}$ yields

$$
\left[\mathrm{R}_{1}^{1}\right]_{w \mathcal{C}}=\left[\mathrm{R}_{(1,0)}^{1}\right]_{w \mathcal{C}}=\left[\mathrm{R}_{1}^{(1,2)}\right]_{w \mathcal{C}}=\left[\mathrm{R}_{(1,0)}^{(1,2)}\right]_{w \mathcal{C}}
$$

These equalities can be read from the lattice of $C$-monoids on $D=\{0,1,2\}$. (cf. Fig. 5.3) 
5.1.10 Corollary. Let $n \geq 3$. Then the number of non-trivial clausal relations to determine all $C$-monoids on $D$ can be reduced from

$$
\frac{1}{4} 4^{n}-2^{n}+1
$$

to

$$
\frac{9}{64} 4^{n}-2^{n-1}+1
$$

Proof. The number of all non-trivial clausal relations $\mathrm{R}_{\mathrm{b}}^{\mathrm{a}}$ where all entries of the tuple $\mathbf{a}$ and of the tuple $\mathbf{b}$ are pairwise distinct, and where all the entries in $\mathbf{a}$ are in ascending order, and in $\mathbf{b}$ are in descending order on $D=\{0, \ldots, n-1\}$ is

$$
\begin{aligned}
|(\mathcal{P}(\{1, \ldots, n-1\}) \backslash\{\emptyset\}) \times(\mathcal{P}(\{0, \ldots, n-2\}) \backslash\{\emptyset\})| & =\left(2^{n-1}-1\right)^{2} \\
& =\frac{1}{4} 4^{n}-2^{n}+1 .
\end{aligned}
$$

From the fist part of Lemma 5.1.9 we consider relations $\mathrm{R}_{\mathbf{d}}^{\mathbf{c}}$, where the parameters $\mathbf{c}$ contain 1 but not 0 , and where the parameters $\mathbf{d}$ contain 0 but not $n-1$, and where $\mathbf{d} \neq(0)$. The number of this kind of relations is $\frac{2^{n-1}}{2} \cdot\left(\frac{2^{n-1}}{2}-1\right)$. From the second part of Lemma 5.1.9, we consider $\mathrm{R}_{\mathbf{d}}^{\mathbf{c}}$, where the parameters $\mathbf{c}$ contain $n-1$ but not 0 , and where the parameters $\mathbf{d}$ contain $n-2$ but not $n-1$ and where $\mathbf{c} \neq(n-1)$. The number of these relations is again $\frac{2^{n-1}}{2} \cdot\left(\frac{2^{n-1}}{2}-1\right)=2^{n-2} \cdot\left(2^{n-2}-1\right)$.

This way some combinations of parameters have been counted twice (those being subject to the first and the second part of Lemma 5.1.9). That are precisely those tuples c containing 1 and $n-1$ but no 0 , and those tuples $\mathbf{d}$ with 0 and $n-2$ but not with $n-1$. The number of those relations is $\left(2^{n-3}\right)^{2}$. This means that the number of relations that are needed to determine all $C$-monoids on $D$ can be reduced to

$$
\begin{aligned}
& \frac{1}{4} 4^{n}-2^{n}+1-2 \cdot 2^{n-2}\left(2^{n-2}-1\right)+\left(2^{n-3}\right)^{2} \\
& =2^{2 n}\left(\frac{2^{4}-2^{3}+1}{2^{6}}\right)-2^{n-1}+1 \\
& =4^{n}\left(\frac{9}{64}\right)-2^{n-1}+1 .
\end{aligned}
$$

The following example shows the reduction of the number of non-trivial clausal relations to determine $C$-monoids on $D=\{0,1,2,3\}$.

5.1.11 Example. Let $D=\{0,1,2,3\}$. At the beginning of the section we explain that to describe all $C$-monoids on $D$, it is sufficient to regard $C$-monoids of the form End $R_{d}^{\mathbf{c}}$, where $R_{\mathbf{d}}^{\mathbf{c}}$ is a non-trivial clausal relation without double parameters. Hence, the set of all non-trivial clausal relations on $D$ without double parameters to regard is:

$$
S:=\left\{\begin{array}{ccccccc}
\mathrm{R}_{0}^{1}, & \mathrm{R}_{1}^{1}, & \mathrm{R}_{2}^{1}, & \mathrm{R}_{(1,0)}^{1}, & \mathrm{R}_{(2,0)}^{1}, & \mathrm{R}_{(2,1)}^{1}, & \mathrm{R}_{(2,1,0)}^{1}, \\
\mathrm{R}_{0}^{2}, & \mathrm{R}_{1}^{2}, & \mathrm{R}_{2}^{2}, & \mathrm{R}_{(1,0)}^{2}, & \mathrm{R}_{(2,0)}^{2}, & \mathrm{R}_{(2,1)}^{2}, & \mathrm{R}_{(2,1,0)}^{2}, \\
\vdots & & & \vdots & & & \vdots \\
\mathrm{R}_{0}^{(1,2,3)}, & \mathrm{R}_{1}^{(1,2,3)}, & \mathrm{R}_{2}^{(1,2,3)}, & \mathrm{R}_{(1,0)}^{(1,2,3)}, & \mathrm{R}_{(2,0)}^{(1,2,3)}, & \mathrm{R}_{(2,1)}^{(1,2,3)}, & \mathrm{R}_{(2,1,0)}^{(1,2,3)}
\end{array}\right\} .
$$


and its cardinality is:

$$
|S|=|(\mathcal{P}(\{1,2,3\}) \backslash\{\emptyset\}) \times(\mathcal{P}(\{0,1,2\}) \backslash\{\emptyset\})|=\left(2^{3}-1\right)^{2}=\frac{1}{4} 4^{4}-2^{4}+1=49 .
$$

The first part of Lemma 5.1.9 states the equality of the $C$-monoids End $\mathrm{R}_{\mathbf{b}}^{1, \mathbf{a}}$ and End $\mathrm{R}_{\mathbf{b}, 0}^{1, \mathbf{a}}$. Hence we look for relations $\mathrm{R}_{\mathbf{d}}^{\mathbf{c}}=\mathrm{R}_{\mathbf{b}, 0}^{1, \mathbf{a}} \in S$, where $\mathbf{c}$ contains 1 but not 0 , and $\mathbf{d}$ contains 0 but not 3 . Furthermore, since $q \geq 1$ we are not going to consider tuples, where $\mathbf{d}=(0)$. The set of these relations is

$$
A=\left\{\begin{array}{cccccc}
\mathrm{R}_{(1,0)}^{1}, & \mathrm{R}_{(2,0)}^{1}, & \mathrm{R}_{(2,1,0)}^{1}, & \mathrm{R}_{(1,0)}^{(1,2)}, & \mathrm{R}_{(2,0)}^{(1,2)}, & \mathrm{R}_{(2,1,0)}^{(1,2)}, \\
\mathrm{R}_{(1,0)}^{(1,3)}, & \mathrm{R}_{(2,0)}^{(1,3)}, & \mathrm{R}_{(2,1,0)}^{(1,3)}, & \mathrm{R}_{(1,0)}^{(1,2,3)}, & \mathrm{R}_{(2,0)}^{(1,2,3)}, & \mathrm{R}_{(2,1,0)}^{(1,2,3)}
\end{array}\right\} .
$$

From the second part of Lemma 5.1.9 we take a look at the relations relations $\mathrm{R}_{\mathbf{d}}^{\mathbf{e}}=\mathrm{R}_{(2, \mathbf{b})}^{(\mathbf{a}, 3)} \in S$ where e contains 3 but not 0 , and $\mathbf{d}$ contains 2 but not 3 , and $\mathbf{e} \neq(3)$. The set of these relations is:

$$
B=\left\{\begin{array}{cccccc}
\mathrm{R}_{2}^{(1,3)}, & \mathrm{R}_{(2,0)}^{(1,3)}, & \mathrm{R}_{(2,1)}^{(1,3)}, & \mathrm{R}_{(2,1,0)}^{(1,3)}, & \mathrm{R}_{2}^{(2,3)}, & \mathrm{R}_{(2,0)}^{(2,3)} \\
\mathrm{R}_{(2,1)}^{(2,3)}, & \mathrm{R}_{(2,1,0)}^{(2,3)}, & \mathrm{R}_{2}^{(1,2,3)}, & \mathrm{R}_{(2,0)}^{(1,2,3)}, & \mathrm{R}_{(2,1)}^{(1,2,3)}, & \mathrm{R}_{(2,1,0)}^{(1,2,3)}
\end{array}\right\} .
$$

Now some relations have been considered twice, namely those relations $R_{\mathbf{d}}^{\mathbf{c}}$ where $\mathbf{c}$ contains 1 and 3 and where $\mathbf{d}$ contains 0 and 2 . These are

$$
C=\left\{\mathrm{R}_{(2,0)}^{(1,3)}, \mathrm{R}_{(2,1,0)}^{(1,3)}, \mathrm{R}_{(2,0)}^{(1,2,3)}, \mathrm{R}_{(2,1,0)}^{(1,2,3)}\right\}
$$

The number of relations to describe $C$-monoids on $D=\{0,1,2,3\}$ can be reduced from 49 to $29=|S|-|A|-|B|+|C|$. Relations that are not crossed out in the scheme below are sufficient to determine all $C$-monoids for $|D|=4$.

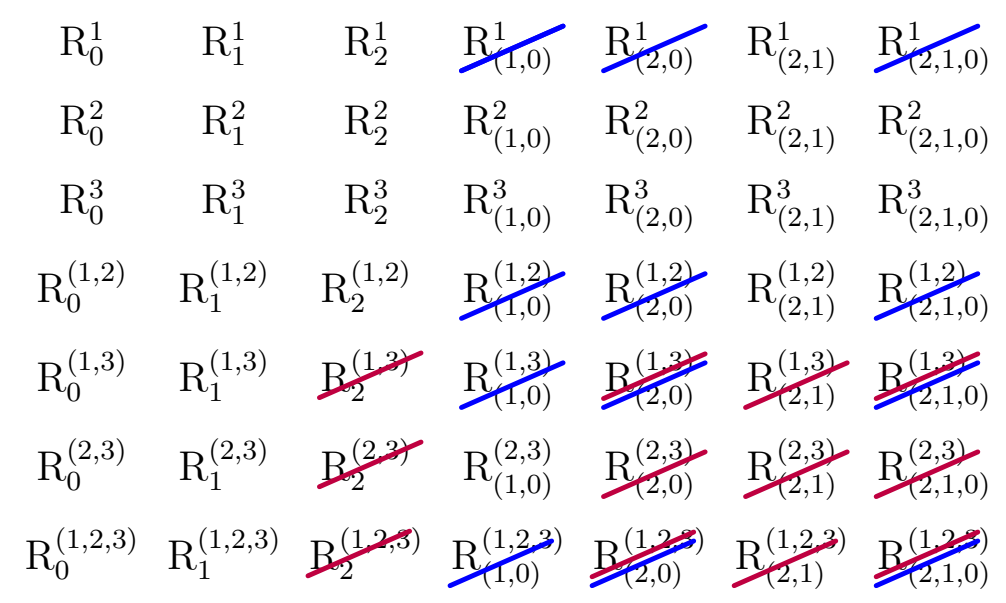

In the next section we investigate the lattice of all $C$-monoids for $|D|=3$. 


\section{$5.2 C$-monoids for $|D|=3$}

In the following we present the Hasse diagram of the lattice $\left(C L_{\mathbf{3}}^{(1)}, \subseteq\right)$, where $C L_{\mathbf{3}}^{(1)}$ is the set of all $C$-monoids on $\mathbf{3}:=\{0,1,2\}$.

Due to Propositions 5.1.4, 5.1.5 and Corollary 5.1.6 the problem of determining all $C$-monoids on the three element set is reduced to the task which functions $f \in \mathrm{O}_{\mathbf{3}}^{(1)}$ (See Table 5.1) preserve which non-trivial clausal relations $\mathrm{R}_{\mathrm{b}}^{\mathrm{a}}$, where all entries of the tuple $\mathbf{a}$ and of the tuple $\mathbf{b}$ are pairwise distinct respectively, and where all the entries in $\mathbf{a}$ are in ascending order, and in $\mathbf{b}$ are in descending order. That is, $\mathbf{R}_{\mathbf{b}}^{\mathbf{a}}$ belongs to

$$
\left\{\mathrm{R}_{0}^{1}, \mathrm{R}_{0}^{2}, \mathrm{R}_{1}^{1}, \mathrm{R}_{1}^{2}, \mathrm{R}_{(1,0)}^{2}, \mathrm{R}_{(1,0)}^{1}, \mathrm{R}_{0}^{(1,2)}, \mathrm{R}_{1}^{(1,2)}, \mathrm{R}_{(1,0)}^{(1,2)}\right\}
$$

In the following tables, the index $k$ of the functions is just the interpretation of $f_{k}(0) f_{k}(1) f_{k}(2)$ as a 3 -adic number: $k=\sum_{i=0}^{2}|\mathbf{3}|^{2-i} \cdot f_{k}(i)$. For example

$$
17=3^{2} \cdot f_{17}(0)+3^{1} \cdot f_{17}(1)+3^{0} \cdot f_{17}(2)
$$

\begin{tabular}{|c|c|c|c|c|c|c|c|c|c|c|c|c|c|c|}
\hline & $f_{0}$ & $f_{1}$ & $f_{2}$ & $f_{3}$ & $f_{4}$ & $f_{5}$ & $f_{6}$ & $f_{7}$ & $f_{8}$ & $f_{9}$ & $f_{10}$ & $f_{11}$ & $f_{12}$ & $f_{13}$ \\
\hline 0 & 0 & 0 & 0 & 0 & 0 & 0 & 0 & 0 & 0 & 1 & 1 & 1 & 1 & 1 \\
1 & 0 & 0 & 0 & 1 & 1 & 1 & 2 & 2 & 2 & 0 & 0 & 0 & 1 & 1 \\
2 & 0 & 1 & 2 & 0 & 1 & 2 & 0 & 1 & 2 & 0 & 1 & 2 & 0 & 1 \\
\hline
\end{tabular}

\begin{tabular}{|c|c|c|c|c|c|c|c|c|c|c|c|c|c|}
\hline & $f_{14}$ & $f_{15}$ & $f_{16}$ & $f_{17}$ & $f_{18}$ & $f_{19}$ & $f_{20}$ & $f_{21}$ & $f_{22}$ & $f_{23}$ & $f_{24}$ & $f_{25}$ & $f_{26}$ \\
\hline 0 & 1 & 1 & 1 & 1 & 2 & 2 & 2 & 2 & 2 & 2 & 2 & 2 & 2 \\
1 & 1 & 2 & 2 & 2 & 0 & 0 & 0 & 1 & 1 & 1 & 2 & 2 & 2 \\
2 & 2 & 0 & 1 & 2 & 0 & 1 & 2 & 0 & 1 & 2 & 0 & 1 & 2 \\
\hline
\end{tabular}

Figure 5.1: Tables of unary functions on $\mathbf{3}$.

Next example provides us with the lattice of all $C$-monoids on $\mathbf{3}$.

5.2.1 Example. The following cross table shows which $f \in \mathrm{O}_{\mathbf{3}}^{(1)}$ preserve which relations $\varrho$ belonging to $\left\{\mathrm{R}_{0}^{1}, \mathrm{R}_{0}^{2}, \mathrm{R}_{1}^{1}, \mathrm{R}_{1}^{2}, \mathrm{R}_{(1,0)}^{2}, \mathrm{R}_{(1,0)}^{1}, \mathrm{R}_{0}^{(1,2)}, \mathrm{R}_{1}^{(1,2)}, \mathrm{R}_{(1,0)}^{(1,2)}\right\}$. The rows are labelled by functions $f_{k}$ (see Table 5.1), the columns are labeled by the relations themselves. Furthermore, in the case when $f \not \varrho$ we give a tuple $\mathbf{r}$ that belongs to $\varrho$ but where $f[\mathbf{r}] \notin \varrho$. For example: In the third row, third column, we have that $(2,2) \in \mathrm{R}_{0}^{2}$. However, $\left(f_{1}(2), f_{1}(2)\right)=(1,1) \notin \mathrm{R}_{0}^{2}$. 


\begin{tabular}{|c|c|c|c|c|c|c|c|c|c|}
\hline$\triangleright$ & $\mathrm{R}_{0}^{1}$ & $\mathrm{R}_{0}^{2}$ & $\mathrm{R}_{1}^{1}$ & $\mathrm{R}_{1}^{2}$ & $\mathrm{R}_{(1,0)}^{2}$ & $\mathrm{R}_{(1,0)}^{1}$ & $\mathrm{R}_{0}^{(1,2)}$ & $\mathrm{R}_{1}^{(1,2)}$ & $\mathrm{R}_{(1,0)}^{(1,2)}$ \\
\hline \hline$f_{0}$ & $\times$ & $\times$ & $\times$ & $\times$ & $\times$ & $\times$ & $\times$ & $\times$ & $\times$ \\
\hline$f_{1}$ & $(1,2)$ & $(2,2)$ & $\times$ & $\times$ & $\times$ & $\times$ & $(1,0,2)$ & $\times$ & $\times$ \\
\hline$f_{2}$ & $(1,2)$ & $\times$ & $(1,2)$ & $\times$ & $\times$ & $(1,2,2)$ & $(1,0,2)$ & $(1,1,2)$ & $(1,1,2,2)$ \\
\hline$f_{3}$ & $(2,1)$ & $(2,1)$ & $\times$ & $\times$ & $\times$ & $\times$ & $(2,2,1)$ & $\times$ & $\times$ \\
\hline$f_{4}$ & $\times$ & $(2,2)$ & $\times$ & $\times$ & $\times$ & $\times$ & $(0,2,1)$ & $\times$ & $\times$ \\
\hline$f_{5}$ & $\times$ & $\times$ & $\times$ & $\times$ & $\times$ & $\times$ & $\times$ & $\times$ & $\times$ \\
\hline$f_{6}$ & $(2,1)$ & $(2,1)$ & $(0,1)$ & $(0,1)$ & $(2,2,1)$ & $(2,1,1)$ & $(2,2,1)$ & $(2,2,1)$ & $(2,2,1,1)$ \\
\hline$f_{7}$ & $\times$ & $(2,2)$ & $(0,1)$ & $(0,1)$ & $(0,1,2)$ & $(0,1,1)$ & $(0,2,1)$ & $(0,2,1)$ & $(0,2,1,1)$ \\
\hline$f_{8}$ & $\times$ & $\times$ & $(0,1)$ & $(0,1)$ & $(0,0,1)$ & $(0,1,1)$ & $\times$ & $(0,0,1)$ & $(0,0,1,1)$ \\
\hline$f_{9}$ & $(2,0)$ & $(1,0)$ & $\times$ & $\times$ & $\times$ & $\times$ & $(1,2,0)$ & $\times$ & $\times$ \\
\hline$f_{10}$ & $(1,0)$ & $(0,0)$ & $\times$ & $\times$ & $\times$ & $\times$ & $(1,2,0)$ & $\times$ & $\times$ \\
\hline$f_{11}$ & $(1,2)$ & $(1,2)$ & $(1,2)$ & $\times$ & $(1,2,0)$ & $(1,2,2)$ & $(1,1,0)$ & $(1,1,2)$ & $(1,1,2,2)$ \\
\hline$f_{12}$ & $(2,1)$ & $(2,1)$ & $\times$ & $\times$ & $\times$ & $\times$ & $(2,2,0)$ & $\times$ & $\times$ \\
\hline$f_{13}$ & $\times$ & $(2,2)$ & $\times$ & $\times$ & $\times$ & $\times$ & $\times$ & $\times$ & $\times$ \\
\hline$f_{14}$ & $\times$ & $(0,0)$ & $\times$ & $\times$ & $(1,2,0)$ & $\times$ & $\times$ & $\times$ & $\times$ \\
\hline$f_{15}$ & $(2,1)$ & $(2,1)$ & $(2,1)$ & $(0,1)$ & $(2,1,0)$ & $(2,1,1)$ & $(2,2,1)$ & $(2,2,1)$ & $(2,2,1,1)$ \\
\hline$f_{16}$ & $\times$ & $(0,0)$ & $\times$ & $(0,1)$ & $(2,1,0)$ & $\times$ & $\times$ & $\times$ & $\times$ \\
\hline$f_{17}$ & $\times$ & $(0,0)$ & $\times$ & $(0,1)$ & $(0,1,1)$ & $\times$ & $\times$ & $\times$ & $\times$ \\
\hline$f_{18}$ & $(2,0)$ & $(2,0)$ & $(2,0)$ & $(2,0)$ & $(2,0,0)$ & $(2,0,0)$ & $(2,2,0)$ & $(2,2,0)$ & $(2,2,0,0)$ \\
\hline$f_{19}$ & $(1,2)$ & $(2,0)$ & $(1,0)$ & $(1,0)$ & $(1,0,0)$ & $(1,0,0)$ & $(1,1,0)$ & $(1,1,0)$ & $(1,1,0,0)$ \\
\hline$f_{20}$ & $(1,2)$ & $(1,0)$ & $(1,0)$ & $(1,0)$ & $(1,0,0)$ & $(1,0,0)$ & $(1,1,0)$ & $(1,1,0)$ & $(1,1,0,0)$ \\
\hline$f_{21}$ & $(2,0)$ & $(2,0)$ & $(2,0)$ & $(2,0)$ & $(2,0,0)$ & $(2,0,0)$ & $(2,2,0)$ & $(2,2,0)$ & $(2,2,0,0)$ \\
\hline$f_{22}$ & $\times$ & $(2,0)$ & $\times$ & $(2,0)$ & $(2,0,0)$ & $\times$ & $\times$ & $\times$ & $\times$ \\
\hline$f_{23}$ & $\times$ & $(1,0)$ & $\times$ & $(1,0)$ & $(1,0,0)$ & $\times$ & $\times$ & $\times$ & $\times$ \\
\hline$f_{24}$ & $(2,0)$ & $(2,0)$ & $(2,0)$ & $(2,0)$ & $(2,0,0)$ & $(2,0,0)$ & $(2,2,0)$ & $(2,2,0)$ & $(2,2,0,0)$ \\
\hline$f_{25}$ & $\times$ & $(2,1)$ & $\times$ & $(2,1)$ & $(2,0,0)$ & $\times$ & $\times$ & $\times$ & $\times$ \\
\hline$f_{26}$ & $\times$ & $\times$ & $\times$ & $\times$ & $\times$ & $\times$ & $\times$ & $\times$ & $\times$ \\
\hline
\end{tabular}

Figure 5.2: Table of $C$-monoids on 3.

The previous table was first determined by hand. Later we verified the cross table with a computer program that determines which unary functions on $D$ (for $|D| \leq 5$ ) preserve which non-trivial clausal relations $\mathrm{R}_{\mathbf{b}}^{\mathbf{a}}$, where all entries of the tuple $\mathbf{a}$ and of the tuple $\mathbf{b}$ are pairwise distinct, respectively.

We present only the pseudo code of the procedure, which verifies whether a given unary function $f$ preserves a given relation of the above mentioned type. 


\section{Pseudo code to verify whether $f \triangleright \mathrm{R}_{\mathrm{b}}^{\mathrm{a}}$.}

S1 Given: $n=|D|, p, q \in\{1, \ldots, n-1\}$, a unary function $f$ on $D$, and tuples $\mathbf{a} \in(D \backslash\{0\})^{p}, \mathbf{b} \in(D \backslash\{n-1\})^{q}$ where all entries of the tuple $\mathbf{a}$ and of $\mathbf{b}$ are pairwise distinct, respectively.

S2 Determine all tuples $\mathbf{r}:=\left(x_{1}, \ldots, x_{p}, y_{1}, \ldots, y_{q}\right) \in D^{p+q}$ that satisfy $x_{1} \geq a_{1} \vee \ldots \vee x_{p} \geq a_{p} \vee y_{1} \leq b_{1} \vee \ldots \vee y_{q} \leq b_{q}$ and collect them in $\mathrm{R}_{\mathrm{b}}^{\mathrm{a}}$.

S3 For each $\mathbf{r} \in \mathrm{R}_{\mathbf{b}}^{\mathbf{a}}$ test whether $f[\mathbf{r}] \in \mathrm{R}_{\mathbf{b}}^{\mathbf{a}}$ holds.

In case it does not, then return $f \not \mathrm{R}_{\mathrm{b}}^{\mathrm{a}}$ and write the tuple $\mathbf{r}$. If all tests were successful, then return $f \triangleright \mathrm{R}_{\mathbf{b}}^{\mathbf{a}}$.

This pseudo code is well-defined because it is an easy application of the Definition 1.3.3. It is complete because the number of tuples in the relation is finite, and all tuples are verified.

Figure 5.3 shows the concept lattice of the formal context (cross table) from Table 5.2. A detailed introduction to concepts, concept lattices, formal contexts can be found in [GW99]. Here these concepts will be not defined.

The pairs of the form $\left(\langle F\rangle_{\mathcal{C O} O_{D}^{(1)}}, C \operatorname{Inv} F\right)$, where $\langle F\rangle_{\mathcal{C O} O_{D}^{(1)}}$ is a $C$-monoid and $C \operatorname{Inv} F$ is the corresponding weak Krasner $C$-clone, and of the form $\left(\operatorname{End} Q,[Q]_{w \mathcal{C}}\right)$, where End $Q$ is a $C$-monoid and $[Q]_{w \mathcal{C}}$ is the corresponding weak Krasner $C$-clone can be read from the diagram.

In the diagram we used the reduced labelling as is common in formal concept analysis (see [GW99]). This means that each function and each clausal relation appear only once in the diagram. The pair of the form $\left(\langle f\rangle_{\mathcal{C} O_{D}^{(1)}}, C \operatorname{Inv} f\right)$ is labelled with $f$, and of the form (End $\mathbf{R}_{\mathbf{b}}^{\mathbf{a}},\left[\mathrm{R}_{\mathbf{b}}^{\mathbf{a}}\right]_{w \mathcal{C}}$ ) is labelled with $\mathbf{R}_{\mathbf{b}}^{\mathbf{a}}$.

For every node in the line diagram one can read the functions belonging to the corresponding $C$-monoid, by collecting all functions located at this node and at nodes which can be reached by descending line paths from this node. For example: The node with label $f_{4}$ corresponds to $\left(\left\langle f_{4}\right\rangle_{\mathcal{C O}}(1), C \operatorname{Inv} f_{4}\right)$. Hence, we get

$$
\left\langle f_{4}\right\rangle_{\mathcal{C O}}(1)=\left\{f_{0}, f_{4}, f_{5}, f_{13}, f_{26}\right\}
$$

Dually, for every node one read the set of relations belonging to the corresponding weak Krasner $C$-clone by following all line paths going upwards from the node and noting down all relations assigned to these nodes. Hence, we obtain

$$
C \operatorname{Inv}\left\{f_{4}\right\}=\left\{\mathrm{R}_{0}^{1}, \mathrm{R}_{1}^{1}, \mathrm{R}_{1}^{2}, \mathrm{R}_{(1,0)}^{2}, \mathrm{R}_{(1,0)}^{1}, \mathrm{R}_{1}^{(1,2)}, \mathrm{R}_{(1,0)}^{(1,2)}\right\}
$$


Similarly, the circle with label $\mathrm{R}_{0}^{(1,2)}$ corresponds to $\left(\right.$ End $\left.\mathrm{R}_{0}^{(1,2)},\left[\mathrm{R}_{0}^{(1,2)}\right]_{w \mathcal{C}}\right)$, where

$$
\begin{aligned}
\text { End } \mathrm{R}_{0}^{(1,2)} & =\left\{f_{0}, f_{5}, f_{8}, f_{13}, f_{14}, f_{16}, f_{17}, f_{22}, f_{23}, f_{25}, f_{26}\right\} \text { and } \\
{\left[\mathrm{R}_{0}^{(1,2)}\right]_{w \mathcal{C}} } & =\left\{\mathrm{R}_{0}^{(1,2)}, \mathrm{R}_{0}^{1}\right\}
\end{aligned}
$$

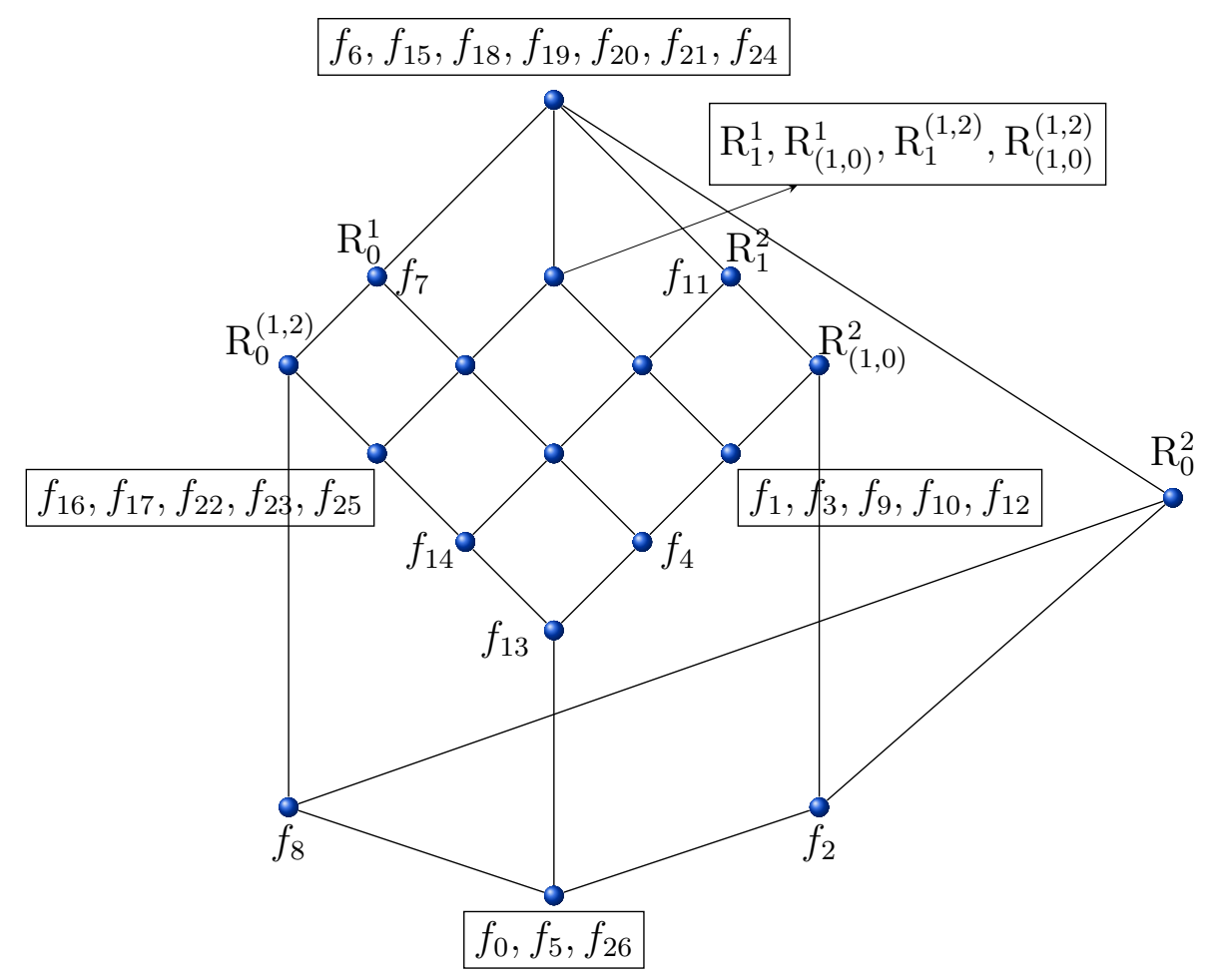

Figure 5.3: The lattice of all $C$-monoids for 3 .

Note that in the lattice that is displayed in Figure 5.3 the co-atoms are of the form; $\langle F\rangle_{\mathcal{C O}}(1)=$ End $\mathrm{R}_{b}^{a}$, where $\mathrm{R}_{b}^{a}$ is a non trivial clausal relation of arity two. In the following section we show that all co-atoms in the lattice of $C$-monoids on an arbitrary finite set $D$ are of this form. Moreover, we describe all atoms in the lattice of $C$-monoids.

\subsection{Maximal $C$-monoids}

In this section all co-atoms in the lattice of $C$-monoids for $|D| \geq 3$ are described. These are formally defined as follows.

5.3.1 Definition. A $C$-monoid $F$ is called maximal if $F \neq \mathrm{O}_{D}^{(1)}$ and between $F$ and $\mathrm{O}_{D}^{(1)}$ there are no more $C$-monoids. That is, for every $C$-monoid $F^{\prime}$ the condition $F \varsubsetneqq F^{\prime}$ implies $F^{\prime}=\mathrm{O}_{D}^{(1)}$. 
Theorem 5.3.5 below, given also in [Var12], states that a $C$-monoid is maximal if it can be determined by only one non-trivial clausal relation of arity two. To prove this theorem we need some auxiliary statements such as Lemmata 5.3.3, 5.3.4 and Remark 5.3.2. The latter states that $C$-monoids of the form End $\mathrm{R}_{b}^{a}$, where $a \in D \backslash\{0\}$ and $b \in D \backslash\{n-1\}$, are candidates for maximal $C$-monoids.

5.3.2 Remark. Let $a \in D \backslash\{0\}$ and $b \in D \backslash\{n-1\}$. Then

$$
\text { End } \mathrm{R}_{b}^{a} \varsubsetneqq \mathrm{O}_{D}^{(1)} \text {. }
$$

Proof. Let $f$ be the unary operation on $D$ defined by $f(x):=n-1-x$. We show that $f \notin$ End $\mathrm{R}_{b}^{a}$. Clearly, the tuple $\mathbf{r}:=(n-1,0)$ belongs to $\mathrm{R}_{b}^{a}$. Moreover, as $0 \nsupseteq a$ and $n-1 \not \leq b$ we obtain $(0, n-1)=f[\mathbf{r}] \notin \mathrm{R}_{b}^{a}$.

5.3.3 Lemma. Let $f \in \mathrm{O}_{D}^{(1)}, a_{1}, \ldots, a_{p} \in D \backslash\{0\}$ and $b_{1}, \ldots, b_{q} \in D \backslash\{n-1\}$. Then we have

$$
f \triangleright \mathrm{R}_{b_{1}, \ldots, b_{q}}^{a_{1}, \ldots, a_{p}} \Rightarrow f \triangleright \mathrm{R}_{\max \left\{b_{1}, \ldots, b_{q}\right\}}^{\min \left\{a_{1}, \ldots, a_{p}\right\}} .
$$

Proof. Let $f \in \mathrm{O}_{D}^{(1)}$ and $f \triangleright \mathrm{R}_{b_{1}, \ldots, b_{q}}^{a_{1}, \ldots, a_{p}}$. We have to show that $f$ preserves $\mathrm{R}_{\max \left\{b_{1}, \ldots, b_{q}\right\}}^{\min \left\{a_{1}, \ldots, a_{p}\right\}}$. Let $\mathbf{r}=\left(x_{1}, y_{1}\right) \in \mathrm{R}_{\max \left\{b_{1}, \ldots, b_{q}\right\}}^{\min \left\{a_{1}, \ldots, a_{p}\right\}}$, i.e.

$$
\begin{gathered}
x_{1} \geq \min \left\{a_{1}, \ldots, a_{p}\right\} \vee y_{1} \leq \max \left\{b_{1}, \ldots, b_{q}\right\} \\
\Longleftrightarrow x_{1} \geq a_{1} \vee \ldots \vee x_{1} \geq a_{p} \vee y_{1} \leq b_{1} \vee y_{1} \leq b_{2} \vee \ldots \vee y_{1} \leq b_{q}
\end{gathered}
$$

Because of $(5.2)$, the tuple $\left(x_{1}, \ldots, x_{1}, y_{1}, \ldots, y_{1}\right)$ belongs to $\mathrm{R}_{b_{1}, \ldots, b_{q}}^{a_{1}, \ldots, a_{p}}$. Furthermore, since $f \triangleright \mathrm{R}_{b_{1}, \ldots, b_{q}}^{a_{1}, \ldots, a_{p}}$, we obtain

$$
\begin{aligned}
& f\left(x_{1}\right) \geq a_{1} \vee \ldots \vee f\left(x_{1}\right) \geq a_{p} \vee f\left(y_{1}\right) \leq b_{1} \vee \ldots \vee f\left(y_{1}\right) \leq b_{q} \\
\Longleftrightarrow & f\left(x_{1}\right) \geq \min \left\{a_{1}, \ldots, a_{p}\right\} \vee f\left(y_{1}\right) \leq \max \left\{b_{1}, \ldots, b_{q}\right\} .
\end{aligned}
$$

Hence, we get $f[\mathbf{r}]=\left(f\left(x_{1}\right), f\left(y_{1}\right)\right) \in \mathrm{R}_{\max \left\{b_{1}, \ldots, b_{q}\right\}}^{\min \left\{a_{1}, \ldots, a_{p}\right\}}$.

We can also prove that any two $C$-monoids, End $\mathrm{R}_{b_{1}}^{a_{1}}$ and End $\mathrm{R}_{b_{2}}^{a_{2}}$, where

$$
\left(a_{1}, b_{1}\right) \neq\left(a_{2}, b_{2}\right)
$$

and $\left(a_{1}, b_{1}\right),\left(a_{2}, b_{2}\right) \in(D \backslash\{0\}) \times(D \backslash\{n-1\})$, are incomparable.

5.3.4 Lemma. Let $a_{1}, a_{2} \in D \backslash\{0\}, b_{1}, b_{2} \in D \backslash\{n-1\}$. If $\left(a_{1}, b_{1}\right) \neq\left(a_{2}, b_{2}\right)$, then we have

$$
\text { End } \mathrm{R}_{b_{1}}^{a_{1}} \nsubseteq \text { End } \mathrm{R}_{b_{2}}^{a_{2}} \quad \text { and } \quad \text { End } \mathrm{R}_{b_{2}}^{a_{2}} \nsubseteq \text { End } \mathrm{R}_{b_{1}}^{a_{1}} \text {. }
$$

Proof. Consider End $\mathrm{R}_{b_{1}}^{a_{1}}$ and End $\mathrm{R}_{b_{2}}^{a_{2}}$, where $\left(a_{1}, b_{1}\right) \neq\left(a_{2}, b_{2}\right)$. We divide the proof in two cases. 
Case 1 If $a_{1} \neq a_{2}$, then w.l.o.g. $a_{1}<a_{2} \leq n-1$. First, we show that End $\mathrm{R}_{b_{1}}^{a_{1}}$ is not contained in End $\mathrm{R}_{b_{2}}^{a_{2}}$. Let $f$ be the unary operation on $D$ defined by

$$
f(x):= \begin{cases}a_{1} & \text { if } x=a_{2}, \\ n-1 & \text { otherwise }\end{cases}
$$

Let $\mathbf{r}:=\left(x_{1}, y_{1}\right) \in \mathrm{R}_{b_{1}}^{a_{1}}$. Since $f\left(x_{1}\right) \geq a_{1}$, we have $f[\mathbf{r}]=\left(f\left(x_{1}\right), f\left(y_{1}\right)\right) \in \mathrm{R}_{b_{1}}^{a_{1}}$ and hence, $f \in$ End $\mathrm{R}_{b_{1}}^{a_{1}}$. Since $\mathbf{r}^{*}:=\left(a_{2}, 0\right) \in \mathrm{R}_{b_{2}}^{a_{2}}$ and $f\left[\mathbf{r}^{*}\right]=\left(a_{1}, n-1\right) \notin \mathrm{R}_{b_{2}}^{a_{2}}$, it follows that $f \notin$ End $\mathrm{R}_{b_{2}}^{a_{2}}$.

To prove End $\mathrm{R}_{b_{2}}^{a_{2}} \nsubseteq$ End $\mathrm{R}_{b_{1}}^{a_{1}}$, consider $g \in \mathrm{O}_{D}^{(1)}$ defined by

$$
g(x):= \begin{cases}0 & \text { if } x=a_{1} \\ x & \text { otherwise }\end{cases}
$$

We show $g \triangleright \mathrm{R}_{b_{2}}^{a_{2}}$. Let $\mathbf{r}=\left(x_{1}, y_{1}\right) \in \mathrm{R}_{b_{2}}^{a_{2}}$, i.e $x_{1} \geq a_{2} \vee y_{1} \leq b_{2}$. We consider two sub-cases:

- If $x_{1} \geq a_{2}>a_{1}$, then $g\left(x_{1}\right)=x_{1} \geq a_{2}$, and $g[\mathbf{r}] \in \mathrm{R}_{b_{2}}^{a_{2}}$.

- If $y_{1} \leq b_{2}$, then $g\left(y_{1}\right) \leq b_{2}$. Hence, $g[\mathbf{r}] \in \mathrm{R}_{b_{2}}^{a_{2}}$.

Since $\mathbf{r}^{\prime}:=\left(a_{1}, n-1\right) \in \mathrm{R}_{b_{1}}^{a_{1}}$ and $g\left[\mathbf{r}^{\prime}\right]=(0, n-1) \notin \mathrm{R}_{b_{1}}^{a_{1}}$, we get $g \not \mathrm{R}_{b_{1}}^{a_{1}}$.

Case 2 If $b_{1} \neq b_{2}$, then w.l.o.g. $0 \leq b_{1}<b_{2}$. First, we prove End $R_{b_{2}}^{a_{2}} \nsubseteq$ End $R_{b_{1}}^{a_{1}}$. Consider $f^{*} \in \mathrm{O}_{D}^{(1)}$ defined by

$$
f^{*}(x):= \begin{cases}b_{2} & \text { if } x=b_{1} \\ 0 & \text { otherwise }\end{cases}
$$

Because $f^{*}\left(y_{1}\right) \leq b_{2}$ for any $\left(x_{1}, y_{1}\right) \in \mathrm{R}_{b_{2}}^{a_{2}}$, we have $f^{*} \triangleright \mathrm{R}_{b_{2}}^{a_{2}}$. Furthermore, $f^{*} \not \mathrm{R}_{b_{1}}^{a_{1}} ;$ since $\left(n-1, b_{1}\right) \in \mathrm{R}_{b_{1}}^{a_{1}}$ and $\left(f^{*}(n-1), f^{*}\left(b_{1}\right)\right) \stackrel{b_{2}}{=}\left(0, b_{2}\right) \notin \mathrm{R}_{b_{1}}^{a_{1}}$. Finally, to prove End $\mathrm{R}_{b_{1}}^{a_{1}} \nsubseteq$ End $\mathrm{R}_{b_{2}}^{a_{2}}$ consider $g^{*} \in \mathrm{O}_{D}^{(1)}$ defined by

$$
g^{*}(x):= \begin{cases}n-1 & \text { if } x=b_{2} \\ x & \text { otherwise }\end{cases}
$$

To show $g^{*} \triangleright \mathrm{R}_{b_{1}}^{a_{1}}$, let $\mathbf{r}=\left(x_{1}, y_{1}\right) \in \mathrm{R}_{b_{1}}^{a_{1}}$, i.e $x_{1} \geq a_{1} \vee y_{1} \leq b_{1}$. We consider two sub-cases:

- If $x_{1} \geq a_{1}$, then $g^{*}\left(x_{1}\right) \geq a_{1}$. Hence, $g^{*}[\mathbf{r}] \in \mathrm{R}_{b_{1}}^{a_{1}}$.

- If $y_{1} \leq b_{1}<b_{2}$, then $g^{*}\left(y_{1}\right)=y_{1} \leq b_{1}$. Hence, we get $g^{*}[\mathbf{r}] \in \mathrm{R}_{b_{1}}^{a_{1}}$.

Moreover, because $\left(0, b_{2}\right) \in \mathrm{R}_{b_{2}}^{a_{2}}$ and $(0, n-1)=\left(g^{*}(0), g^{*}\left(b_{2}\right)\right) \notin \mathrm{R}_{b_{2}}^{a_{2}}$, it follows that $g^{*} \not \mathrm{R}_{b_{2}}^{a_{2}}$.

Now we can characterise all maximal $C$-monoids. 
5.3.5 Theorem. Let $M \subseteq \mathrm{O}_{D}^{(1)}$ be a $C$-monoid. $M$ is maximal if and only if there are elements $a \in D \backslash\{0\}$ and $b \in D \backslash\{n-1\}$ such that

$$
M=\text { End } \mathrm{R}_{b}^{a} \text {. }
$$

Proof. " $\Rightarrow:$ Let $M \subseteq \mathrm{O}_{D}^{(1)}$ be a maximal $C$-monoid. Then there exists a relation $\varrho \in C \mathrm{R}_{D}^{*}$ such that $M=$ End $\varrho$. This means there are elements $a_{1} \ldots a_{p} \in D \backslash\{0\}$ and $b_{1}, \ldots, b_{q} \in D \backslash\{n-1\}$ such that $M=$ End $\mathrm{R}_{b_{1}, \ldots, b_{q}}^{a_{1}, \ldots, a_{p}}$. From 5.3.3 and 5.3.2, we have

$$
M=\text { End } \mathrm{R}_{b_{1}, \ldots, b_{q}}^{a_{1}, \ldots, a_{p}} \stackrel{5.3 .3}{\subseteq} \text { End } \mathrm{R}_{\max \left\{b_{1}, \ldots, b_{q}\right\}}^{\min \left\{a_{1}, \ldots, a_{p}\right\}} \stackrel{5.3 .2}{\subsetneq} \mathrm{O}_{D}^{(1)}
$$

Since $M$ is maximal, we have $M=\operatorname{End} \mathrm{R}_{\max \left\{b_{1}, \ldots, b_{q}\right\}}^{\min \left\{a_{1}, \ldots, a_{p}\right\}}$.

" $\Leftarrow$ :" Let End $\mathrm{R}_{b}^{a}$ be a $C$-monoid, where $a \in D \backslash\{0\}$ and $b \in D \backslash\{n-1\}$. Because of 5.3.2, we have End $\mathrm{R}_{b}^{a} \varsubsetneqq \mathrm{O}_{D}^{(1)}$. Since the lattice of the $C$-monoids is finite, there is a maximal $C$-monoid $M$ such that

$$
\text { End } \mathrm{R}_{b}^{a} \subseteq M \varsubsetneqq \mathrm{O}_{D}^{(1)} .
$$

The first part of this proof implies the existence of elements $c \in D \backslash\{0\}$ and $d \in D \backslash\{n-1\}$ such that $M=$ End $\mathrm{R}_{d}^{c}$. Hence, End $\mathrm{R}_{b}^{a} \subseteq$ End $\mathrm{R}_{d}^{c}$. Because of 5.3.4, we have $a=c$ and $b=d$. Thus, $M=$ End $\mathrm{R}_{b}^{a}$ is maximal.

Finally, from Theorem 5.3.5, we deduce there are $(n-1)^{2}$ maximal $C$-monoids on an $n$-element set $D$, for $|D| \geq 3$.

\subsection{Minimal $C$-monoids}

In this section, all atoms in the lattice of $C$-monoids for $|D| \geq 3$ are described, these are formally defined as follows.

5.4.1 Definition. Let $i d_{D}$ be the identity on $D$. A $C$-monoid $F$ is minimal, if $F \neq\left\langle i d_{D}\right\rangle_{C O_{D}^{(1)}}$ and between $F$ and $\left\langle i d_{D}\right\rangle_{\mathcal{C O}_{D}^{(1)}}$ there are no more $C$-monoids.

We begin the characterisation of all minimal $C$-monoids by describing the smallest $C$-monoid.

5.4.2 Lemma. Let $c_{0}, c_{n-1}$ be unary constants operations with values 0 and $n-1$ respectively, and let $i d_{D}$ be the identity on $D$. Then

$$
\text { End } C \mathrm{R}_{D}=\left\{c_{0}, c_{n-1}, i d_{D}\right\} \text {. }
$$

Proof. We first show $\left\{c_{0}, c_{n-1}, i d_{D}\right\} \subseteq$ End $C \mathrm{R}_{D}$. The identity $i d_{D}$ belongs to End $\mathrm{R}_{D} \subseteq$ End $C \mathrm{R}_{D}$. Furthermore, let $\mathrm{R}_{\mathbf{b}}^{\mathbf{a}} \in C \mathrm{R}_{D}$ with $\mathbf{a} \in D^{p}, \mathbf{b} \in D^{q}$ and consider $\mathbf{r} \in \mathrm{R}_{\mathbf{b}}^{\mathbf{a}}$. Since $0 \leq b_{j}$ and $n-1 \geq a_{j}$, we have $c_{0}[\mathbf{r}]=(0, \ldots, 0) \in \mathrm{R}_{\mathbf{b}}^{\mathbf{a}}$ and $c_{n-1}[\mathbf{r}]=(n-1, \ldots, n-1) \in \mathrm{R}_{\mathbf{b}}^{\mathbf{a}}$. Consequently,

$$
c_{0} \triangleright \mathrm{R}_{\mathbf{b}}^{\mathbf{a}} \text { and } c_{n-1} \triangleright \mathrm{R}_{\mathbf{b}}^{\mathbf{a}} \text {. }
$$


Conversely, we assume that there is a function $f \in\left(\right.$ End $\left.C \mathrm{R}_{D}\right) \backslash\left\{c_{0}, c_{n-1}, i d_{D}\right\}$. Since $f \neq i d_{D}$, there is some $c \in D$ such that $f(c) \neq c$. As $f \neq c_{0}$, there is some $y \in D$ such that $f(y)>0$. Finally, $f \neq c_{n-1}$ implies there is some $x \in D$ such that $f(x)<n-1$. We divide the remaining proof in two cases:

- If $f(c)<c$, then since $(c, y) \in \mathrm{R}_{0}^{c}$ and $(f(c), f(y)) \notin \mathrm{R}_{0}^{c}$, it follows that $f \not \mathrm{R}_{0}^{c}$. This is impossible, since $f \in$ End $C \mathrm{R}_{D}$.

- If $f(c)>c$, then from $(x, c) \in \mathrm{R}_{c}^{n-1}$ and $(f(x), f(c)) \notin \mathrm{R}_{c}^{n-1}$, we receive $f \not \mathrm{R}_{c}^{n-1}$, which is also a contradiction.

Consequently, (End $\left.C \mathrm{R}_{D}\right) \backslash\left\{c_{0}, c_{n-1}, i d_{D}\right\}=\emptyset$, which proves the claim.

In the rest of this section we shall characterise one-generated $C$-monoids for some functions $f \in \mathrm{O}_{D}^{(1)}$ (namely $f \in\left\{f_{a}^{-}, f_{a}^{+}, c_{a} \mid 0<a<n-1\right\}$ defined hereafter).

For $0<a<n-1$ let $f_{a}^{-}, f_{a}^{+}, c_{a} \in \mathrm{O}_{D}^{(1)}$ be the unary operations defined by

$$
f_{a}^{-}(x):=\left\{\begin{array}{ll}
a-1 & \text { if } x=a \\
x & \text { otherwise, }
\end{array} \quad f_{a}^{+}(x):= \begin{cases}a+1 & \text { if } x=a \\
x & \text { otherwise }\end{cases}\right.
$$

and $c_{a}(x):=a$ for all $x \in D$. These functions allow us to characterise the minimal $C$-monoids.

The lemma below states that clausal relations belonging to the weak Krasner $C$-clones of $f_{a}^{-}$are those clausal relations $\mathbf{R}_{\mathbf{b}}^{\mathbf{e}}$ such that $e_{1}, \ldots, e_{p} \notin\{0, a\}$.

5.4.3 Lemma. Let $0<a<n-1$. Then,

$$
C \mathrm{R}_{D}^{*} \cap C \operatorname{Inv} f_{a}^{-}=\left\{\mathrm{R}_{\mathbf{b}}^{\mathbf{e}} \mid \mathbf{e} \in(D \backslash\{0, a\})^{p}, \mathbf{b} \in(D \backslash\{n-1\})^{q}\right\} .
$$

Proof. We define

$$
W:=\left\{\mathrm{R}_{\mathbf{b}}^{\mathbf{e}} \mid \mathbf{e} \in(D \backslash\{0, a\})^{p}, \mathbf{b} \in(D \backslash\{n-1\})^{q}\right\} \subseteq C \mathrm{R}_{D}^{*} .
$$

First, we show $W \subseteq C \operatorname{Inv} f_{a}^{-}$. Let $\mathrm{R}_{\mathbf{b}}^{\mathbf{e}} \in W$, we have to prove that $f_{a}^{-} \triangleright \mathrm{R}_{\mathbf{b}}^{\mathbf{e}}$. By the equation (4.1) before Lemma 4.1.4, it is enough to prove $f_{a}^{-} \triangleright\left[e_{i}, n-1\right]$ for all $i \in\{1, \ldots, p\}$ and $f_{a}^{-} \triangleright\left[0, b_{j}\right]$ for all $j \in\{1, \ldots, q\}$. Let $y \in\left[0, b_{j}\right]$. Since $f_{a}^{-}(y) \leq y \leq b_{j}$, one obtains $f_{a}^{-} \triangleright\left[0, b_{j}\right]$ for all $j \in\{1, \ldots, q\}$.

To prove $f_{a}^{-} \triangleright\left[e_{i}, n-1\right]$ for all $i \in\{1, \ldots, p\}$, let us assume the opposite, i.e., there is one $x \in\left[e_{i}, n-1\right]$ such that $f_{a}^{-}(x) \notin\left[e_{i}, n-1\right]$. The latter implies $f_{a}^{-}(x)<e_{i}$. By definition of $f_{a}^{-}$there are only two cases to consider.

1) If $x=a$, then $a=x \geq e_{i}>f_{a}^{-}(x)=f_{a}^{-}(a)$ and by definition $f_{a}^{-}(a)=a-1$. Consequently, $e_{i}=a$. This is impossible, since $\mathrm{R}_{\mathbf{b}}^{\mathbf{e}} \in W$.

2) If $x \neq a$, then $x=f_{a}^{-}(x)<e_{i}$, a contradiction to $x \in\left[e_{i}, n-1\right]$. 
We show $C \mathrm{R}_{D}^{*} \cap C \operatorname{Inv} f_{a}^{-} \subseteq W$. Assume that there is a relation $\mathrm{R}_{\mathbf{b}^{\prime}}^{\mathbf{e}^{\prime}}$ belonging to $C \operatorname{Inv} f_{a}^{-} \cap C \mathrm{R}_{D}^{*}$ but not to $W$. Hence, $a=e_{i}^{\prime}$ for some $i \in\{1, \ldots, p\}$, i.e., $\mathrm{R}_{\mathbf{b}^{\prime}}^{\mathbf{e}^{\prime}}$ is equal to $\mathrm{R}_{\mathbf{b}^{\prime}}^{\left(e_{1}^{\prime}, \ldots, e_{i-1}^{\prime}, a, e_{i+1}^{\prime}, \ldots, e_{p}^{\prime}\right)}$. Then

$$
\mathbf{r}:=(0, \ldots, 0, \stackrel{i}{a}, 0, \ldots, 0, n-1, \ldots, n-1) \in \mathrm{R}_{\mathbf{b}^{\prime}}^{\left(e_{1}^{\prime}, \ldots, e_{i-1}^{\prime}, a, e_{i+1}^{\prime}, \ldots, e_{p}^{\prime}\right)} .
$$

Since $f_{a}^{-} \triangleright \mathrm{R}_{\mathbf{b}^{\prime}}^{\mathbf{e}^{\prime}}$, we receive $f_{a}^{-}[\mathbf{r}] \in \mathrm{R}_{\mathbf{b}^{\prime}}^{\left(e_{1}^{\prime}, \ldots, e_{i-1}^{\prime}, a, e_{i+1}^{\prime}, \ldots, e_{p}^{\prime}\right)}$. But this yields to a contradiction since

$$
\begin{aligned}
0<e_{1}^{\prime} \wedge \ldots \wedge 0<e_{i-1}^{\prime} \wedge a-1<a \wedge 0<e_{i+1}^{\prime} \wedge & \ldots \wedge 0<e_{p}^{\prime} \\
& \wedge n-1>b_{1}^{\prime} \wedge \ldots \wedge n-1>b_{q}^{\prime} .
\end{aligned}
$$

Similarly, one can prove following statement, which states that clausal relations belonging to the weak Krasner $C$-clones of $f_{a}^{+}$are those clausal relations $\mathrm{R}_{\mathrm{b}}^{\mathrm{e}}$ such that $b_{1}, \ldots, b_{q}$ do not belong to $\{a, n-1\}$.

5.4.4 Lemma. Let $0<a<n-1$. Then,

$$
C \mathrm{R}_{D}^{*} \cap C \operatorname{Inv} f_{a}^{+}=\left\{\mathrm{R}_{\mathbf{b}}^{\mathbf{e}} \mid \mathbf{e} \in(D \backslash\{0\})^{p}, \mathbf{b} \in(D \backslash\{a, n-1\})^{q}\right\} .
$$

We see below that the one-generated $C$-monoids of functions of the form $f_{a}^{-}$or $f_{a}^{+}$are atoms in the lattice of all $C$-monoids. We show for $g \in\left\{f_{a}^{-}, f_{a}^{+}\right\}$that whenever $f$ belongs to $\langle g\rangle_{\mathcal{C} O_{D}^{(1)}}$ and $f$ does not belong to the smallest $C$-monoid, then $f=g$, in particular $\langle f\rangle_{\mathcal{C} O_{D}^{(1)}}=\langle g\rangle_{\mathcal{C} O_{D}^{(1)}}$. To do this we need some additional auxiliary statements.

5.4.5 Lemma. Let $f \in \mathrm{O}_{D}^{(1)}, c \in D$ and let $c_{0}$ and $c_{n-1}$ be unary constants operations with values 0 and $n-1$, respectively. Then the following holds:

1) If $f(c)<c$, then $f=c_{0}$ or $f \not \mathrm{R}_{0}^{c}$.

2) If $f(c)>c$, then $f=c_{n-1}$ or $f \not \mathrm{R}_{c}^{n-1}$.

Proof. Let $f(c)<c$. If $f \neq c_{0}$, then there is some $y \in D$ such that $f(y) \neq 0$. Hence, we get $(c, y) \in \mathrm{R}_{0}^{c}$, and $(f(c), f(y)) \notin \mathrm{R}_{0}^{c}$. Thus, $f \not \mathrm{R}_{0}^{c}$. Similarly, the second statement can be proved.

5.4.6 Corollary. Let $0<a<n-1, c_{0}, c_{n-1}$. Then we have

$$
\begin{aligned}
& g \in\left\langle f_{a}^{-}\right\rangle_{\mathcal{C O}(1)} \Rightarrow g \in\left\{c_{0}, c_{n-1}\right\} \text { or } g(c)=c \text { for all } c \in D \backslash\{a\}, \\
& g \in\left\langle f_{a}^{+}\right\rangle_{\mathcal{C O}}(1) \Rightarrow g \in\left\{c_{0}, c_{n-1}\right\} \text { or } g(c)=c \text { for all } c \in D \backslash\{a\} .
\end{aligned}
$$

Proof. Let $g \in\left\langle f_{a}^{-}\right\rangle_{\mathcal{C O}}(1)$. By Lemma 5.4.3, it follows $g \triangleright \mathrm{R}_{\mathbf{b}}^{\mathbf{e}}$ where $\mathbf{e} \in(D \backslash\{0, a\})^{p}$. Assume that $g \neq c_{0}$ and $g \neq c_{n-1}$, and there is some $c \in D \backslash\{a\}$ such that $g(c) \neq c$. Since $g(c) \neq c$, we consider two cases. 
- If $g(c)<c$, then from Lemma 5.4.5 we infer $g \not \mathrm{R}_{0}^{c}$ as $g \neq c_{0}$. However, as $g \in$ End $C \operatorname{Inv} f_{a}^{-}$and $\mathrm{R}_{0}^{c} \in C \operatorname{Inv} f_{a}^{-}$, we obtain $g \triangleright \mathrm{R}_{0}^{c}$, which is a contradiction.

- Similarly, if $g(c)>c$, then $g \not \mathrm{R}_{c}^{n-1}$ because $g \neq c_{n-1}$. As $g \in \operatorname{End} C \operatorname{Inv} f_{a}^{-}$ and $\mathrm{R}_{c}^{n-1} \in C \operatorname{Inv} f_{a}^{-}$, we obtain $g \triangleright \mathrm{R}_{c}^{n-1}$, which is also a contradiction.

Similarly, one can prove the second statement.

Finally, we obtain the following corollary.

5.4.7 Corollary. Let $0<a<n-1, c_{0}, c_{n-1}$ and $i d_{D}$. Then we have

1) If $f \in\left(\right.$ End $\left.C \operatorname{Inv} f_{a}^{-}\right) \backslash\left\{c_{0}, c_{n-1}, i d_{D}\right\}$, then $f=f_{a}^{-}$.

2) If $f \in\left(\right.$ End $\left.C \operatorname{Inv} f_{a}^{+}\right) \backslash\left\{c_{0}, c_{n-1}, i d_{D}\right\}$, then $f=f_{a}^{+}$.

Proof. Let $f \in\left(\right.$ End $\left.C \operatorname{Inv} f_{a}^{-}\right) \backslash\left\{c_{0}, c_{n-1}, i d_{D}\right\}$. We show that $f(a)=a-1$ and $f(x)=x$ for $x \in D \backslash\{a\}$. The preconditions, together with Corollary 5.4.6, imply that $f(c)=c$ for all $c \neq a$. As $f \neq i d_{D}, n-1 \geq d:=f(a) \neq a$. Assume that $d>a$. Since $f \neq c_{n-1}$ and because of 5.4.5, we infer $f \not \mathrm{R}_{a}^{n-1}$. This is impossible, since $\mathrm{R}_{a}^{n-1} \in C \operatorname{Inv} f_{a}^{-}$and $f \in$ End $C \operatorname{Inv} f_{a}^{-}$. Thus, $d<a$. Assume $d<a-1$. As $f \neq c_{0}$, there is some $y \in D$ such that $f(y) \supsetneqq 0$. Obviously, $(a, y) \in \mathrm{R}_{0}^{a-1}$ but $(f(a), f(y)) \notin \mathrm{R}_{0}^{a-1}$. Hence, we get $f \not \mathrm{R}_{0}^{a-1}$. This is impossible, since $f \in$ End $C \operatorname{Inv} f_{a}^{-}$and $\mathrm{R}_{0}^{a-1}$ belongs to $C \operatorname{Inv} f_{a}^{-}$. Hence, $f(a)=d=a-1$. Thus, $f=f_{a}^{-}$. Similarly, the second statement can be proved.

Proposition 5.4 .8 below, given also in [Var12], states that $\left\langle c_{a}\right\rangle_{\mathcal{C} O_{D}^{(1)}}, 0<a<n-1$ are minimal and describes their weak Krasner $C$-clones.

5.4.8 Proposition. Let $0<a<n-1$ and $c_{a}$ be the unary constant operation with value a. Then we have

$$
C \mathrm{R}_{D}^{*} \cap C \operatorname{Inv}\left(c_{a}\right)=\left\{\mathrm{R}_{\mathbf{d}}^{\mathbf{e}} \mid \exists i \in\{1, \ldots, p\}: e_{i} \leq a \text { or } \exists j \in\{1, \ldots, q\}: d_{j} \geq a\right\},
$$

and

$$
\text { End } C \operatorname{Inv}\left(c_{a}\right)=\left\{i d_{D}, c_{0}, c_{n-1}, c_{a}\right\} \text {, i.e. }\left\langle c_{a}\right\rangle_{\mathcal{C O}_{D}^{(1)}} \text { is minimal. }
$$

Proof. We define

$$
W:=\left\{\mathrm{R}_{\mathbf{d}}^{\mathbf{e}} \mid \exists i \in\{1, \ldots, p\}: e_{i} \leq a \text { or } \exists j \in\{1, \ldots, q\}: d_{j} \geq a\right\} \subseteq C \mathrm{R}_{D}^{*} .
$$

Since $c_{a}\left[\left(x_{1}, \ldots, x_{p}, y_{1}, \ldots, y_{q}\right)\right]=(a, \ldots, a) \in \mathrm{R}_{\mathbf{d}}^{\mathbf{e}}$ for all $\mathrm{R}_{\mathbf{d}}^{\mathbf{e}} \in W$, we have

$$
C \operatorname{Inv}\left(c_{a}\right) \supseteq W
$$

In order to prove $W \supseteq C \operatorname{Inv} c_{a} \cap C \mathrm{R}_{D}^{*}$, we assume that there is a relation $\mathrm{R}_{\mathbf{d}^{\prime}}^{\mathbf{e}^{\prime}}$ belonging to $C \operatorname{Inv}\left(c_{a}\right) \cap C \mathrm{R}_{D}^{*}$ but not to $W$. The latter implies $a<e_{i}^{\prime}$ for all $i \in\{1, \ldots, p\}$ and $d_{j}^{\prime}<a$ for all $j \in\{1, \ldots, q\}$. Let $\mathbf{r} \in \mathrm{R}_{\mathbf{d}^{\prime}}^{\mathbf{e}^{\prime}}$. We have $c_{a}[\mathbf{r}]=(a, \ldots, a) \notin \mathrm{R}_{\mathbf{d}^{\prime}}^{\mathbf{e}^{\prime}}$, 
which is a contradiction to $c_{a} \triangleright \mathrm{R}_{\mathbf{d}^{\prime}}^{\mathbf{e}^{\prime}}$

We show End $C \operatorname{Inv}\left(c_{a}\right)=\left\{i d_{D}, c_{0}, c_{n-1}, c_{a}\right\}$. The inclusion " $\supseteq$ " is trivial. Conversely, take $f \in \operatorname{End} C \operatorname{Inv}\left(c_{a}\right)$. Assume $f \neq c_{0}, f \neq c_{n-1}$ and $f \neq i d_{D}$. Since $f$ is not $i d_{D}$, there is some $c \in D$ such that $f(c) \neq c$. As $f \neq c_{0}$, there is some $y \in D$ such that $f(y)>0$. Finally, $f \neq c_{n-1}$ implies that there is some $x \in D$ such that $f(x)<n-1$. For the rest of the proof we show $f=c_{a}$.

1) For all $d \in[a, n-1]$ and for all $z \in[0, d]$ we have $f(z) \leq d$.

Let $d \in[a, n-1]$ and choose any element $z \in[0, d]$. As $z \leq d$, it follows $(x, z) \in \mathrm{R}_{d}^{n-1}$. Since $d \geq a$, we receive $\mathrm{R}_{d}^{n-1} \in C \operatorname{Inv} c_{a}$ and hence, $f \triangleright \mathrm{R}_{d}^{n-1}$. Thus, $(f(x), f(z)) \in \mathrm{R}_{d}^{n-1}$. For $f(x)<n-1$, it follows $f(z) \leq d$.

2) For all $e \in[0, a]$ and for all $z \in[e, n-1]$ we have $f(z) \geq e$.

The proof is analogous to 1 ).

3) For all $d \in[a, n-1]$ it holds that $f(d) \leq d$, and for all $e \in[0, a]$ we have $f(e) \geq e$. In particular, $f(a)=a$.

The first statement is a special case of 1 ), the second one a special case of 2). A combination of the two latter results yields $f(a)=a$.

4) For all $z \in[0, a]$ we have $f(z) \leq a$, and for all $z \in[a, n-1]$ we have $f(z) \geq a$. The claims follow from 1) and 2), respectively.

5) For all $z \in[0, a]$ it holds that $z \leq f(z) \leq a$, and for all $z \in[a, n-1]$ we have $a \leq f(z) \leq z$.

This is a combination of 3 ) and 4).

6) For all $z \in[0, a)$ such that $f(z) \neq z$ we have $f(z)=a$.

Let $z \in[0, a)$ and $f(z) \neq z$, then by 5) we obtain $z<f(z) \leq a$. Obviously, $(z, z) \in \mathrm{R}_{z}^{a}$. Since $\mathrm{R}_{z}^{a} \in C \operatorname{Inv} c_{a}$, we get $f \triangleright \mathrm{R}_{z}^{a}$. Thus, $(f(z), f(z)) \in \mathrm{R}_{z}^{a}$. Since $f(z)>z$, it follows $f(z) \geq a$.

7) For all $z \in(a, n-1]$ such that $f(z) \neq z$, we have $f(z)=a$.

The proof is analogous to 6 )

8) For all $z \in D$, it holds that $f(z)=z$ or $f(z)=a$.

This is a combination of 3),6) and 7).

9) There exists a $v \in D \backslash\{a\}$ such that $f(v)=a$.

We assume, $f \neq i d_{D}$. Hence, so there is some $v \in D$ such that $f(v) \neq v$. Statement 3) implies that $v \neq a$, and from 8) we infer $f(v)=a$.

10) If $f(0)=a$, then $f(z) \leq a$ for all $z \in D$.

Let $f(0)=a$ and $z \in D$. Then it holds that $(a, 0, z) \in \mathrm{R}_{(0, a)}^{n-1}$, and $\mathrm{R}_{(0, a)}^{n-1}$ belongs to $C \operatorname{Inv} c_{a}$. Hence, we get $(a, a, f(z))=(f(a), f(0), f(z)) \in \mathrm{R}_{(0, a)}^{n-1}$. Since $0<a<n-1$, it follows $f(z) \leq a$. 
11) If $f(n-1)=a$, then $f(z) \geq a$ for all $z \in D$.

The proof is analogous to 10 ).

12) If there exists a $v \in[0, a)$ such that $f(v)=a$, then $f=c_{a}$.

Let $v \in[0, a)$ such that $f(v)=a$. Let $z \in D$, then $(z, v) \in \mathrm{R}_{v}^{a}$. Since $\mathrm{R}_{v}^{a} \in C \operatorname{Inv} c_{a}$, we receive $f \triangleright \mathrm{R}_{v}^{a}$. Hence $(f(z), a)=(f(z), f(v)) \in \mathrm{R}_{v}^{a}$. As $a>v$, we infer

$$
f(z) \geq a .
$$

Now 4) implies $f(z) \leq a$ for all $z \in[0, a]$. This yields the precondition of 10$)$. Hence, we can infer $f(z) \leq a$ for all $z \in D$. That together with $(\star)$, implies $f=c_{a}$.

13) Now we can prove $f=c_{a}$.

In case that the precondition of 12) is fulfilled, we are done. Otherwise, from $9)$ we infer $f(v)=a$ for some $v \in(a, n-1]$. For any $z \in D$ we have that $(v, z) \in \mathrm{R}_{a}^{v} \in C \operatorname{Inv} c_{a}$. Since $f \triangleright \mathrm{R}_{a}^{v}$, it follows $(a, f(z)) \in \mathrm{R}_{a}^{v}$. Because of $a<v$, we get

$$
f(z) \leq a .
$$

Now 5) implies $a \leq f(z)$ for all $z \in[a, n-1]$. This yields the precondition of 11). Hence, it follows $f(z) \geq a$ for all $z \in D$. That together with $(\star \star)$ yields to $f=c_{a}$.

We finish this section by proving that there are no other minimal $C$-monoids than the ones already described in the previous Lemmata 5.4.3, 5.4.4 and Proposition 5.4.8. To do this we need an additional auxiliary statement.

5.4.9 Lemma. Let $f \in \mathrm{O}_{D}^{(1)}$ be an unary operation and $f \notin\left\{c_{0}, c_{n-1}\right\}$. If for every $a \in\{1, \ldots, n-2\}$ there exists $\varrho_{a} \notin C \operatorname{Inv} c_{a}$ such that $f \triangleright \varrho_{a}$, then $f(0)=0$ and $f(n-1)=n-1$.

Proof. Let $f \in \mathrm{O}_{D}^{(1)} \backslash\left\{c_{0}, c_{n-1}\right\}$. To prove $f(0)=0$ and $f(n-1)=n-1$, let us assume $f(0) \neq 0$ or $f(n-1) \neq n-1$. First we assume $f(0)>0$, we consider two cases:

- If $f(0)=: a \in\{1,2, \ldots, n-2\}$, then there exists a $\varrho_{a} \notin C \operatorname{Inv} c_{a}$ and $f \triangleright \varrho_{a}$. Since $\varrho_{a} \notin C \operatorname{Inv} c_{a}$, the parameters of the relation $\varrho_{a}=\mathrm{R}_{d_{1}, \ldots, d_{q}}^{e_{1}, \ldots, e_{p}}$ fulfil $a<e_{i}$ for all $i \in\{1, \ldots, p\}$ and $a>d_{j}$ for all $j \in\{1, \ldots, q\}$. Since $0 \leq d_{1}$, the tuple $\mathbf{r}:=(0, \ldots, \stackrel{p}{0}, \ldots, \stackrel{p+q}{0})$ belongs to $\varrho_{a}$, but $f[\mathbf{r}]=(a, \ldots, \stackrel{p}{a}, \ldots, \stackrel{p+q}{a}) \notin \varrho_{a}$. This contradicts $f \triangleright \varrho_{a}$.

- If $f(0)=n-1$, then $f \neq c_{n-1}$ implies that there is some $x \in D \backslash\{0\}$ such that $f(x)<n-1$. Let $f(x)=: b \in\{0,1, \ldots, n-2\}$, again we consider two subcases.

1. If $b \in\{1, \ldots, n-2\}$, then similar to the first case we obtain a contradiction to $f \triangleright \varrho_{b}$ for some $\varrho_{b} \notin C \operatorname{Inv} c_{b}$. 
2. Let $f(x)=b=0$ for $x \in D \backslash\{0\}$. By assumption and since $|D| \geq 3$, there exists $\varrho_{1}$ such that $\varrho_{1} \notin C \operatorname{Inv} c_{1}$ and $f \triangleright \varrho_{1}$. Since $\varrho_{1} \notin C \operatorname{Inv} c_{1}$, the relation $\varrho_{1}=\mathrm{R}_{d_{1}, \ldots, d_{q}}^{e_{1}, \ldots, e_{p}}$ satisfies $1<e_{i}$ for all $i \in\{1, \ldots, p\}$ and $1>d_{j}$ for all $j \in\{1, \ldots, q\}$. The tuple $\mathbf{r}^{*}:=(x, \ldots, \stackrel{p}{x}, 0, \ldots, \stackrel{p+q}{0})$ belongs to $\varrho_{1}$ because of $0 \leq d_{1}$. However, we receive

$$
f\left[\mathbf{r}^{*}\right]=(0, \ldots, \stackrel{p}{0}, n-1, \ldots, n-1) \notin \varrho_{1}^{p+q},
$$

in contradiction to $f \triangleright \varrho_{1}$.

Thus, $f(0)=0$. Similarly, we can prove that $f(n-1)=n-1$.

5.4.10 Theorem. For all $f \in \mathrm{O}_{D}^{(1)} \backslash\left\{i d_{D}, c_{0}, c_{n-1}\right\}$ there exists $a \in\{1, \ldots, n-2\}$ such that

$$
\begin{array}{rll}
C \operatorname{Inv} f \subseteq C \operatorname{Inv} c_{a}, & \text { i.e. } & \left\langle c_{a}\right\rangle_{\mathcal{C O}}^{(1)} \subseteq\langle f\rangle_{D} O_{D}^{(1)}, \\
\text { or } C \operatorname{Inv} f \subseteq C \operatorname{Inv} f_{a}^{-}, & \text {i.e. } & \left\langle f_{a}^{-}\right\rangle_{\mathcal{C O}}(1) \subseteq\langle f\rangle_{D} O_{D}^{(1)} \\
\text { or } C \operatorname{Inv} f \subseteq C \operatorname{Inv} f_{a}^{+}, & \text {i.e. } & \left\langle f_{a}^{+}\right\rangle_{\mathcal{C O}(1)} \subseteq\langle f\rangle_{\mathcal{C} O_{D}^{(1)}}
\end{array}
$$

Proof. Let $f \in \mathrm{O}_{D}^{(1)} \backslash\left\{i d_{D}, c_{0}, c_{n-1}\right\}$. Assume that for all $a \in\{1, \ldots, n-2\}$ it holds that

$\begin{array}{llll} & C \operatorname{Inv} f & \nsubseteq & C \operatorname{Inv} c_{a}, \\ \text { and } & C \operatorname{Inv} f & \nsubseteq & C \operatorname{Inv} f_{a}^{-}, \\ \text {and } & C \operatorname{Inv} f & \nsubseteq & C \operatorname{Inv} f_{a}^{+} .\end{array}$

Hence, we infer that for all $a \in\{1, \ldots, n-2\}$ it holds that

$$
\begin{aligned}
& \exists \varrho_{a} \notin C \operatorname{Inv} c_{a}: f \triangleright \varrho_{a}, \\
& \text { and } \exists \varrho_{a}^{-} \notin C \operatorname{Inv} f_{a}^{-}: f \triangleright \varrho_{a}^{-} \text {, } \\
& \text { and } \exists \varrho_{a}^{+} \notin C \operatorname{Inv} f_{a}^{+} \quad: \quad f \triangleright \varrho_{a}^{+} \text {. }
\end{aligned}
$$

As for all $a \in\{1, \ldots, n-2\}$ there is some $\varrho_{a} \notin C \operatorname{Inv} c_{a}$ such that $f \triangleright \varrho_{a}$, we infer from Lemma 5.4.9 that $f(n-1)=n-1$ and $f(0)=0$.

Since for all $a \in\{1, \ldots, n-2\}$ there is some $\varrho_{a}^{-} \in C \operatorname{Inv} f$ such that $f_{a}^{-} \not \varrho_{a}^{-}$, we obtain from Lemma 5.4.3 that

$$
\varrho_{a}^{-}=\mathrm{R}_{\mathbf{b}}^{e_{1}, \ldots, e_{i-1}, a, e_{i+1}, \ldots, e_{p}}
$$

for some $i \in\{1, \ldots, p\}, e_{k} \neq 0$ for $k \neq i$, and $\mathbf{b} \in(D \backslash\{n-1\})^{q}$. The tuple

$$
\mathbf{r}:=(0, \ldots, 0, \stackrel{i}{a}, 0, \ldots, \stackrel{p}{0}, n-1, \ldots, n-1)
$$

belongs to $\varrho_{a}^{-}$. Since $f \triangleright \varrho_{a}^{-}$, we receive

$$
(0, \ldots, 0, f(a), 0, \ldots, 0, n-1, \ldots, n-1)=f[\mathbf{r}] \in \varrho_{a}^{-},
$$


and hence,

$$
f(a) \geq a
$$

As for all $a \in\{1, \ldots, n-2\}$ there is some $\varrho_{a}^{+} \in C \operatorname{Inv} f$ such that $f_{a}^{+} \not \varrho_{a}^{+}$, we obtain from Lemma 5.4.4 that

$$
\varrho_{a}^{+}=\mathrm{R}_{b_{1}, \ldots, b_{j-1}, a, b_{j+1}, \ldots, b_{q}}^{\mathbf{e}}
$$

for some $j \in\{1, \ldots, q\}, b_{k} \neq n-1$ for $k \neq j$ and $\mathbf{e} \in(D \backslash\{0\})^{p}$. Let

$$
\mathbf{r}^{*}:=(0, \ldots, \stackrel{p}{0}, n-1, \ldots, n-1, \stackrel{j}{a}, n-1, \ldots, n-1) \in \varrho_{a}^{+} .
$$

Since $f \triangleright \varrho_{a}^{+}$, we receive

$$
f\left[\mathbf{r}^{*}\right]=(0, \ldots, 0, n-1, \ldots, n-1, f(a), n-1, \ldots, n-1) \in \varrho_{a}^{+} .
$$

Hence,

$$
f(a) \leq a
$$

We have $f(a)=a$ for all $a \in\{1, \ldots, n-2\}$, due to the Inequalities (5.3) and (5.4). Furthermore, $f(0)=0$ and $f(n-1)=n-1$. Thus, $f=i d_{D}$. This is impossible, since $f \notin\left\{c_{0}, c_{n-1}, i d_{D}\right\}$.

To finish this section, we present all results obtained so far in the Figure 5.4. Here we see the following:

- The rectangle labeled as $\left\langle f_{a}^{-}\right\rangle_{\mathcal{C} O_{D}^{(1)}}, 0<a<n-1$, denotes minimal $C$-monoids that are generated by $f_{a}^{-}$, where $0<a<n-1$.

- The rectangle labeled as $\left\langle f_{a}^{+}\right\rangle_{\mathcal{C O}}(1), 0<a<n-1$, denotes minimal $C$-monoids that are generated by $f_{a}^{+}$, where $0<a<n-1$.

- The rectangle labeled as $\left\langle c_{a}^{(1)}\right\rangle_{\mathcal{C O}_{D}^{(1)}}, 0<a<n-1$, denotes minimal $C$-monoids that are generated by the unary constant operation $c_{a}^{(1)}$ with value $a$, where $a \in\{1, \ldots, n-2\}$.

There are $n-2$ different minimal $C$-monoids on $D$, with $|D| \geq 3$, of the form $\left\langle f_{a}^{-}\right\rangle_{\mathcal{C O}(1)}, n-2$ of the form $\left\langle f_{a}^{+}\right\rangle_{\mathcal{C} O_{D}^{(1)}}$, and $n-2$ of the form $\left\langle c_{a}^{(1)}\right\rangle_{\mathcal{C} O_{D}^{(1)}}$. Furthermore, due to Theorem 5.4.10 there are no other minimal $C$-monoids than the ones already described. Thus, there are $3(n-2)$ minimal $C$-monoids on a $n$-element set $D$, where $|D| \geq 3$. 


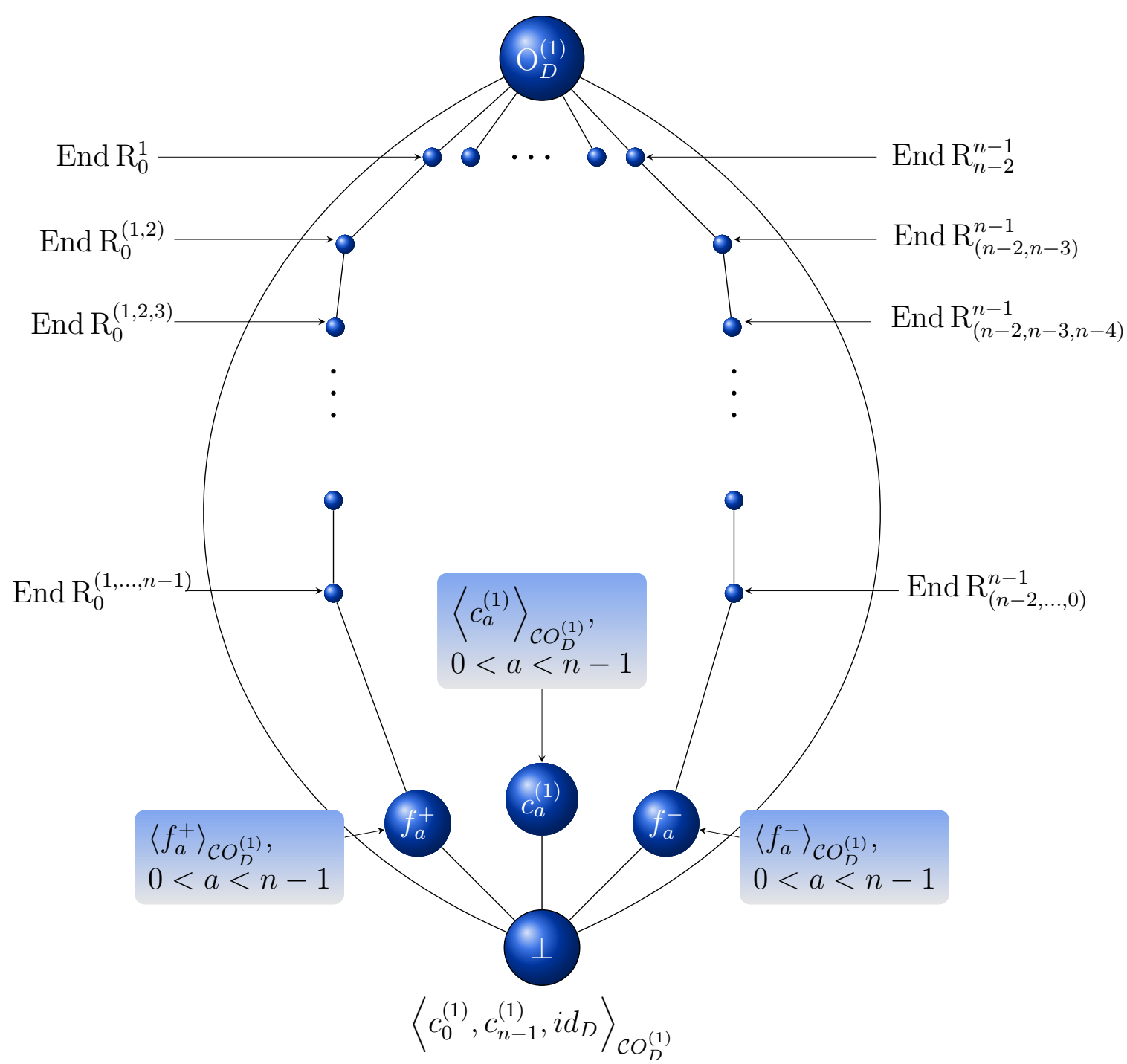

Figure 5.4: The lattice of $C$-monoids for $|D| \geq 3$.

\subsection{Special $C$-monoids for $|D| \geq 3$}

In this section some $C$-monoids and weak Krasner $C$-clones on $D$, where $|D| \geq 3$, are described. Hereafter, the image of $f$ is denoted by $\operatorname{Im}(f)$.

5.5.1 Lemma. Let $k \in\{1, \ldots, n-2\}$ be fixed, $F:=\left\{f \in O_{D}^{(1)} \mid \operatorname{Im}(f) \subseteq\{0, \ldots, k\}\right\}$. Then

$$
C \mathrm{R}_{D}^{*} \cap C \operatorname{Inv} F=\left\{\mathrm{R}_{\mathbf{b}}^{\mathbf{a}} \in C \mathrm{R}_{D}^{*} \mid \exists j \in\{1, \ldots, q\}: b_{j} \geq k\right\} .
$$

Proof. Fix $k \in\{1, \ldots, n-2\}$. We define

$$
W:=\left\{\mathrm{R}_{\mathrm{b}}^{\mathrm{a}} \in C \mathrm{R}_{D}^{*} \mid \exists j \in\{1, \ldots, q\}: b_{j} \geq k\right\} \subseteq C \mathrm{R}_{D}^{*} .
$$


First, we show $W \subseteq C \operatorname{Inv} F$. Let $\varrho \in W$ and $f \in F$, we have to prove that $f \triangleright \varrho$. Since $\varrho \in W$, we get $\varrho=\mathrm{R}_{\mathbf{b}}^{\mathbf{a}} \in C \mathrm{R}_{D}^{*}$ with $b_{j} \geq k$ for some $j \in\{1, \ldots, q\}$. Consider any tuple $\mathbf{r}:=\left(x_{1}, \ldots, x_{p}, y_{1}, \ldots, y_{q}\right)$ belonging to $\varrho$. Since $\operatorname{Im}(f) \subseteq\{0, \ldots, k\}$, we obtain $f\left(y_{j}\right) \leq k \leq b_{j}$. Thus, $f[\mathbf{r}] \in \varrho$.

To prove $W \supseteq C \mathrm{R}_{D}^{*} \cap C \operatorname{Inv} F$, we assume there is a relation $\mathrm{R}_{\mathbf{b}^{\prime}}^{\mathbf{a}^{\prime}}$ belonging to $C \mathrm{R}_{D}^{*} \cap C \operatorname{Inv} F$ but not to $W$. Hence, the parameters of the relation $\mathrm{R}_{\mathbf{b}^{\prime}}^{\mathbf{a}^{\prime}}$ fulfill $b_{j}^{\prime}<k$ for all $j \in\{1, \ldots, q\}$. Consider the unary function $f_{k}$ defined by

$$
f_{k}(x):= \begin{cases}k & \text { if } x=0 \\ 0 & \text { otherwise }\end{cases}
$$

By definition, $f_{k}$ belongs to $F$. Furthermore, the tuple $\left(n-1, \ldots, n-\frac{p}{-} 1, \stackrel{1}{0}, \ldots, \stackrel{q}{0}\right)$ belongs to $\mathrm{R}_{\mathbf{b}^{\prime}}^{\mathbf{a}^{\prime}}$. Since $f_{k} \triangleright \mathrm{R}_{\mathbf{b}^{\prime}}^{\mathbf{a}^{\prime}}$, we obtain

$$
\left(f_{k}(n-1), \ldots, f_{k}(n-1), f_{k}(0), \ldots, f_{k}(0)\right)=(0, \ldots, 0, k, \ldots, k) \in \mathrm{R}_{\mathbf{b}^{\prime}}^{\mathbf{a}^{\prime}} .
$$

However, this yields to a contradiction since $0 \nsupseteq a_{i}^{\prime}$ for all $i \in\{1, \ldots, p\}$ and $k \not \leq b_{j}^{\prime}$ for all $j \in\{1, \ldots, q\}$.

If $D=\{0,1,2\}$ and $k=1$, then $F=\left\{f \in \mathrm{O}_{D}^{(1)} \mid \operatorname{Im}(f) \subseteq\{0,1\}\right\}$ is equal to $\left\{f_{1}, f_{3}, f_{4}, f_{9}, f_{10}, f_{12}, f_{13}\right\}$ (see Table 5.1). From Lemma 5.5.1 we infer

$$
\begin{aligned}
C \mathrm{R}_{D}^{*} \cap C \operatorname{Inv} F & =\left\{\mathrm{R}_{\mathbf{b}}^{\mathbf{a}} \in C \mathrm{R}_{D}^{*} \mid \exists j \in\{1, \ldots, q\}: b_{j} \geq 1\right\} \\
& =\left\{\mathrm{R}_{1}^{1}, \mathrm{R}_{1}^{2}, \mathrm{R}_{(1,0)}^{2}, \mathrm{R}_{(1,0)}^{1}, \mathrm{R}_{1}^{(1,2)}, \mathrm{R}_{(1,0)}^{(1,2)}\right\} .
\end{aligned}
$$

The last equality can be read from the lattice of all $C$-monoids on $D=\{0,1,2\}$ depicted in the Figure 5.3.

Similarly, the following lemma can be proved.

5.5.2 Lemma. Let $k \in\{1, \ldots, n-2\}$ be fixed, and let

$$
F:=\left\{f \in \mathrm{O}_{D}^{(1)} \mid \operatorname{Im}(f) \subseteq\{k, \ldots, n-1\}\right\} .
$$

Then, $C \mathrm{R}_{D}^{*} \cap C \operatorname{Inv} F=\left\{\mathrm{R}_{\mathbf{b}}^{\mathbf{a}} \in C \mathrm{R}_{D}^{*} \mid \exists i \in\{1, \ldots, p\}: a_{i} \leq k\right\}$.

If $D=\{0,1,2\}$ and $k=1$, then $F=\left\{f \in \mathrm{O}_{D}^{(1)} \mid \operatorname{Im}(f) \subseteq\{1,2\}\right\}$ is equal to $\left\{f_{13}, f_{14}, f_{16}, f_{17}, f_{22}, f_{23}, f_{25}, f_{26}\right\}$, and from Lemma 5.5.2 we infer

$$
C \mathrm{R}_{D}^{*} \cap C \operatorname{Inv} F=\left\{\mathrm{R}_{1}^{1}, \mathrm{R}_{0}^{1}, \mathrm{R}_{0}^{(1,2)}, \mathrm{R}_{(1,0)}^{1}, \mathrm{R}_{1}^{(1,2)}, \mathrm{R}_{(1,0)}^{(1,2)}\right\} .
$$

5.5.3 Lemma. Let $k \in\{1, \ldots, n-2\}$, and $s \in\{1, \ldots, k\}$ be fixed, let $D$ be the domain with at least four elements. Let also $F:=\left\{f \in \mathrm{O}_{D}^{(1)} \mid \operatorname{Im}(f) \subseteq\{s, \ldots, k\}\right\}$. Then,

$$
\begin{aligned}
C \mathrm{R}_{D}^{*} \cap C \operatorname{Inv} F= & \left\{\mathrm{R}_{\mathbf{b}}^{\mathbf{a}} \in C \mathrm{R}_{D}^{*} \mid \exists i \in\{1, \ldots, p\}: a_{i} \leq s\right\} \cup \\
& \left\{\mathrm{R}_{\mathbf{b}}^{\mathbf{a}} \in C \mathrm{R}_{D}^{*} \mid \exists j \in\{1, \ldots, q\}: b_{j} \geq k\right\}
\end{aligned}
$$


Proof. Fix $k \in\{1, \ldots, n-2\}$ and $s \in\{1, \ldots, k\}$. We define

$$
\begin{aligned}
& W_{1}:=\left\{\mathrm{R}_{\mathbf{b}}^{\mathbf{a}} \in C \mathrm{R}_{D}^{*} \mid \exists i \in\{1, \ldots, p\}: a_{i} \leq s\right\} \subseteq C \mathrm{R}_{D}^{*}, \\
& W_{2}:=\left\{\mathrm{R}_{\mathbf{b}}^{\mathbf{a}} \in C \mathrm{R}_{D}^{*} \mid \exists j \in\{1, \ldots, q\}: b_{j} \geq k\right\} \subseteq C \mathrm{R}_{D}^{*} .
\end{aligned}
$$

First, we show $W_{1} \cup W_{2} \subseteq C \operatorname{Inv} F$. Let $\varrho \in W_{1} \cup W_{2}$ and $f \in F$, we have to prove that $f \triangleright \varrho$. Since $\varrho \in W_{1} \cup W_{2}$, we consider two cases:

- If $\varrho \in W_{1}$, then $\varrho=\mathrm{R}_{\mathbf{b}}^{\mathbf{a}}$ with $a_{i} \leq s$ for some $i \in\{1, \ldots, p\}$. Consider any tuple $\mathbf{r}:=\left(x_{1}, \ldots, x_{p}, y_{1}, \ldots, y_{q}\right)$ belonging to $\varrho$. Because $\operatorname{Im}(f) \subseteq\{s, \ldots, k\}$, we get $f\left(x_{i}\right) \geq s \geq a_{i}$. Thus, $f[\mathbf{r}] \in \varrho$.

- If $\varrho \notin W_{1}$, then $\varrho$ belongs to $W_{2}$. The latter implies $\varrho=\mathrm{R}_{\mathbf{b}}^{\mathbf{a}}$ with $b_{j} \geq k$ for some $j \in\{1, \ldots, q\}$. For any tuple $\mathbf{r}=\left(x_{1}, \ldots, x_{p}, y_{1}, \ldots, y_{q}\right)$ belonging to $\varrho$, we have $f[\mathbf{r}] \in \varrho$, since $f\left(y_{j}\right) \leq k \leq b_{j}$.

We show $W_{1} \cup W_{2} \supseteq C \mathrm{R}_{D}^{*} \cap C$ Inv $F$. Assume there is a relation $\mathrm{R}_{\mathbf{b}^{\prime}}^{\mathbf{a}^{\prime}}$ belonging to $C \mathrm{R}_{D}^{*} \cap C \operatorname{Inv} F$ but not to $W_{1} \cup W_{2}$. Hence, the parameters of $\mathrm{R}_{\mathbf{b}^{\prime}}^{\mathbf{a}^{\prime}}$ fulfill $a_{i}^{\prime}>s$ for all $i \in\{1, \ldots, p\}$ and $b_{j}^{\prime}<k$ for all $j \in\{1, \ldots, q\}$. Let $f_{k}^{s}$ be the unary function defined by

$$
f_{k}^{s}(x):= \begin{cases}s & \text { if } x=n-1 \\ k & \text { otherwise }\end{cases}
$$

By definition, the function $f_{k}^{s}$ belongs to $F$. Since $(n-1, \ldots, n \stackrel{p}{-} 1, \stackrel{1}{0}, \ldots, \stackrel{q}{0}) \in \mathrm{R}_{\mathbf{b}^{\prime}}^{\mathbf{a}^{\prime}}$. and $f_{k}^{s} \triangleright \mathrm{R}_{\mathbf{b}^{\prime}}^{\mathbf{a}^{\prime}}$, we obtain

$$
\left(f_{k}^{s}(n-1), \ldots, f_{k}^{s}(n-1), f_{k}^{s}(0), \ldots, f_{k}^{s}(0)\right)=(s, \ldots, s, k, \ldots, k) \in \mathrm{R}_{\mathbf{b}^{\prime}}^{\mathbf{a}^{\prime}} .
$$

However, this yields to a contradiction since $a_{i}^{\prime}>s$ for all $i \in\{1, \ldots, p\}$ and $b_{j}^{\prime}<k$ for all $j \in\{1, \ldots, q\}$.

For what is left of this section, we will characterise one-generated $C$-monoids for some functions $f \in \mathrm{O}_{D}^{(1)}$, (namely $f \in\left\{g_{k}, g_{k}^{\prime} \mid 0<k<n-1\right\}$ ) defined hereafter. For $k \in\{1, \ldots, n-2\}$, let $g_{k}, g_{k}^{\prime}$ be the unary operations on $D$ given by

$$
g_{k}(x):=\min \left(c_{k}(x), x\right) \text { and } \quad g_{k}^{\prime}(x):=\max \left(c_{k}(x), x\right),
$$

where $c_{k}$ is the unary constant operation with value $k$.

The $C$-monoids generated by these functions allow us to characterise some upper covers of the minimal $C$-monoids generated by $c_{k}$, where $k \in\{1, \ldots, n-2\}$.

5.5.4 Lemma. Let $k \in\{1, \ldots, n-2\}$ be fixed, $c_{k}$ be the unary constant operation with value $k$, and let $g_{k}$ be the unary operation on $D$ defined by

$$
g_{k}(x):=\min \left(c_{k}(x), x\right) .
$$

Then we have

$$
C \mathrm{R}_{D}^{*} \cap C \operatorname{Inv} g_{k}=\left\{\mathrm{R}_{\mathbf{b}}^{\mathbf{a}} \mid \forall i \in\{1, \ldots, p\}: a_{i} \leq k \text { or } \exists j \in\{1, \ldots, q\}: b_{j} \geq k\right\} .
$$


Proof. Let $k \in\{1, \ldots, n-2\}$ be fixed. We define

$$
W:=\left\{\mathrm{R}_{\mathbf{b}}^{\mathbf{a}} \mid \forall i \in\{1, \ldots, p\}: a_{i} \leq k \text { or } \exists j \in\{1, \ldots, q\}: b_{j} \geq k\right\} \subseteq C \mathrm{R}_{D}^{*} .
$$

First, we show $W \subseteq C \operatorname{Inv} g_{k}$. Let $\varrho \in W$ and let $\mathbf{r}:=\left(x_{1}, \ldots, x_{p}, y_{1}, \ldots, y_{q}\right)$ be any tuple belonging to $\varrho$. Since $\varrho \in W$, we get $\varrho=\mathrm{R}_{\mathbf{b}}^{\mathbf{a}}$, where $b_{j} \geq k$ for some $j \in\{1, \ldots, q\}$ or $a_{i} \leq k$ for all $i \in\{1, \ldots, p\}$. We consider the following cases:

- If there is $j \in\{1, \ldots, q\}$ such that $b_{j} \geq k$, then, since $\operatorname{Im}\left(g_{k}\right) \in\{1, \ldots, k\}$, we get $g_{k}\left(y_{j}\right) \leq k \leq b_{j}$. Thus, $g_{k}[\mathbf{r}] \in \varrho$.

- Otherwise, for every $j \in\{1, \ldots, q\}$ we get $b_{j}<k$. Then because $\varrho \in W$, we get $a_{i} \leq k$ for all $i \in\{1, \ldots, p\}$. Since $\mathbf{r} \in \varrho$, one obtains

$$
x_{1} \geq a_{1} \vee \ldots \vee x_{p} \geq a_{p} \vee y_{1} \leq b_{1} \vee \ldots \vee y_{q} \leq b_{q},
$$

and we consider the following subcases:

1. If $y_{j} \leq b_{j}$ for some $j \in\{1, \ldots, q\}$, then $y_{j} \leq b_{j}<k$, and hence, $g_{k}\left(y_{j}\right)=y_{j} \leq b_{j}$. Consequently, $g_{k}[\mathbf{r}] \in \varrho$.

2. If $x_{i} \geq a_{i} \in\{1, \ldots, k\}$ for some $i \in\{1, \ldots, p\}$, then either the element $x_{i}$ belongs to $\{1, \ldots, k\}$ or $x_{i}>k$. If $x_{i} \in\{1, \ldots, k\}$, then $g_{k}\left(x_{i}\right)=x_{i} \geq a_{i}$, and hence $g_{k}[\mathbf{r}] \in \varrho$. If $x_{i}>k$, then $g_{k}\left(x_{i}\right)=k \geq a_{i}$, and likewise $g_{k}[\mathbf{r}] \in \varrho$.

Second, we show $C \mathrm{R}_{D}^{*} \cap C \operatorname{Inv} g_{k} \subseteq W$. Assume there is a relation $\varrho$ belonging to $C \mathrm{R}_{D}^{*} \cap C \operatorname{Inv} g_{k}$ but not to $W$. Hence, $\varrho=\mathrm{R}_{\mathbf{b}^{\prime}}^{\mathbf{e}^{\prime}}$, where $e_{i}^{\prime}>k$ for some $i \in\{1, \ldots, p\}$ and $b_{j}^{\prime}<k$ for all $j \in\{1, \ldots, q\}$. The tuple $\mathbf{r}:=(0, \ldots, 0, n \stackrel{i}{-} 1,0, \ldots, \stackrel{p}{0}, \stackrel{1}{k}, \ldots, \stackrel{q}{k})$ belongs to $\varrho$. Since $g_{k} \triangleright \mathrm{R}_{\mathbf{b}^{\prime}}^{\mathbf{e}^{\prime}}$, we have $g_{k}[\mathbf{r}] \in \mathrm{R}_{\mathbf{b}^{\prime}}^{\mathbf{e}^{\prime}}$. However, this yields a contradiction since $g_{k}[\mathbf{r}]=(0, \ldots, 0, \stackrel{i}{k}, 0, \ldots, \stackrel{p}{0}, \stackrel{1}{k}, \ldots, \stackrel{q}{k})$ and

$$
\begin{aligned}
0<e_{1}^{\prime} \wedge \ldots \wedge 0<e_{i-1}^{\prime} \wedge k<e_{i}^{\prime} \wedge 0<e_{i+1}^{\prime} \wedge \ldots \wedge 0<e_{p}^{\prime} & \\
& \wedge k>b_{1}^{\prime} \wedge \ldots \wedge k>b_{q}^{\prime} .
\end{aligned}
$$

The dual statement of 5.5.4 is the following.

5.5.5 Lemma. Let $k \in\{1, \ldots, n-2\}$ be fixed, $c_{k}$ be the unary constant operation with value $k$, and let $g_{k}^{\prime}$ be the unary operation on $D$, defined by

$$
g_{k}^{\prime}(x):=\max \left(c_{k}(x), x\right) .
$$

Then we have

$$
C \mathrm{R}_{D}^{*} \cap C \operatorname{Inv} g_{k}^{\prime}=\left\{\mathrm{R}_{\mathbf{b}}^{\mathbf{a}} \mid \exists i \in\{1, \ldots, p\}: a_{i} \leq k \text { or } \forall j \in\{1, \ldots, q\}: b_{j} \geq k\right\} .
$$


In following propositions we establish that the one-generated $C$-monoids of functions $g_{k}$ or $g_{k}^{\prime}$ for fixed $k \in\{1, \ldots, n-2\}$, are upper covers of $\left\langle c_{k}\right\rangle_{\mathcal{C} O_{D}^{(1)}}$ in the lattice of all $C$-monoids.

5.5.6 Proposition. Let $k \in\{1, \ldots, n-2\}$ be fixed, and let $g_{k}$ be the unary operation given by $g_{k}(x)=\min \left(c_{k}(x), x\right)$ for $x \in D$. Then

$$
\left\langle g_{k}\right\rangle_{\mathcal{C O}(1)}=\left\{g_{k}, c_{k}, c_{0}, c_{n-1}, i d_{D}\right\}=\left\langle c_{0}, c_{n-1}, g_{k}\right\rangle_{O_{D}^{(1)}}
$$

Proof. First, we show $\left\{g_{k}, c_{k}, c_{0}, c_{n-1}, i d_{D}\right\}=\left\langle c_{0}, c_{n-1}, g_{k}\right\rangle_{O_{D}^{(1)}}$. Clearly,

$$
\left\{g_{k}, c_{0}, c_{n-1}, i d_{D}\right\} \subseteq\left\langle c_{0}, c_{n-1}, g_{k}\right\rangle_{O_{D}^{(1)}} .
$$

Furthermore, as $c_{k}(x)=\min \left(c_{k}\left(c_{n-1}(x)\right), c_{n-1}(x)\right)=g_{k} \circ c_{n-1}(x)$, we have that $c_{k}$ belongs to $\left\langle c_{0}, c_{n-1}, g_{k}\right\rangle_{O_{D}^{(1)}}$. Thus, $\left\{g_{k}, c_{0}, c_{n-1}, i d_{D}, c_{k}\right\} \subseteq\left\langle c_{0}, c_{n-1}, g_{k}\right\rangle_{O_{D}^{(1)}}$.

Let $F:=\left\{i d_{D}, c_{0}, c_{k}, c_{n-1}, g_{k}\right\}$. Because $F$ belongs to $\left\langle c_{0}, c_{n-1}, g_{k}\right\rangle_{O_{D}^{(1)}}$, in order to calculate all functions that belong to $\left\langle c_{0}, c_{n-1}, g_{k}\right\rangle_{O_{D}^{(1)}}$, we first calculate all compositions $x \circ y$ between functions $x, y$ of $F$.

\begin{tabular}{c|ccccc}
$x \backslash y$ & $i d_{D}$ & $c_{0}$ & $c_{k}$ & $c_{n-1}$ & $g_{k}$ \\
\hline \hline$i d_{D}$ & $i d_{D}$ & $c_{0}$ & $c_{k}$ & $c_{n-1}$ & $g_{k}$ \\
$c_{0}$ & $c_{0}$ & $c_{0}$ & $c_{k}$ & $c_{n-1}$ & $c_{0}$ \\
$c_{k}$ & $c_{k}$ & $c_{0}$ & $c_{k}$ & $c_{n-1}$ & $c_{k}$ \\
$c_{n-1}$ & $c_{n-1}$ & $c_{0}$ & $c_{k}$ & $c_{n-1}$ & $c_{k}$ \\
$g_{k}$ & $g_{k}$ & $c_{0}$ & $c_{k}$ & $c_{n-1}$ & $g_{k}$
\end{tabular}

From the previous table we obtain that $F$ is closed under composition of fuctions, i.e., there are no more functions in $\left\langle c_{0}, c_{n-1}, g_{k}\right\rangle_{O_{D}^{(1)}}$ than those belonging to $F$. Consequently, $\left\langle c_{0}, c_{n-1}, g_{k}\right\rangle_{O_{D}^{(1)}}=F=\left\{i d_{D}, c_{0}, c_{k}, c_{n-1}, g_{k}\right\}$.

Second, we show $\left\langle g_{k}\right\rangle_{\mathcal{C} O_{D}^{(1)}}=\left\langle c_{0}, c_{n-1}, g_{k}\right\rangle_{O_{D}^{(1)}}$. We have $\left\langle c_{0}, c_{n-1}, g_{k}\right\rangle_{O_{D}^{(1)}} \subseteq\left\langle g_{k}\right\rangle_{\mathcal{C O}} O_{D}^{(1)}$. In order to show $\left\langle g_{k}\right\rangle_{\mathcal{C} O_{D}^{(1)}} \subseteq\left\langle c_{0}, c_{n-1}, g_{k}\right\rangle_{O_{D}^{(1)}}$, we will prove that

$$
\text { if } g \in\left\langle g_{k}\right\rangle_{\mathcal{C O}(1)} \backslash\left\{c_{0}, c_{n-1}, c_{k}, i d_{D}\right\} \text {, then } g=g_{k} .
$$

Take $g \in$ End $C \operatorname{Inv}\left(g_{k}\right)=\left\langle g_{k}\right\rangle_{\mathcal{C} O_{D}^{(1)}}$. Suppose $g \neq c_{0}, g \neq c_{k}, g \neq c_{n-1}$ and $g \neq i d_{D}$. Since $g$ is not $i d_{D}$, there is some $c \in D$ such that $g(c) \neq c$. As $g \neq c_{0}$, there is some $y \in D$ such that $g(y)>0$, and $g \neq c_{n-1}$ implies that there is some $x \in D$ such that $g(x)<n-1$. Finally, as $g \neq c_{k}$ there is some $d \in D$ such that $g(d) \neq k$. For the rest of the proof we show $g=g_{k}$.

1) For all $z \in[0, k]$ we have $g(z) \leq k$.

Let $z \in[0, k]$. As $z \leq k$, it follows $(x, z) \in \mathrm{R}_{k}^{n-1}$. From Lemma 5.5.4, we infer that the relation $\mathrm{R}_{k}^{n-1}$ belongs to $C \operatorname{Inv} g_{k}$, and hence $g \triangleright \mathrm{R}_{k}^{n-1}$. Thus, $(g(x), g(z)) \in \mathrm{R}_{k}^{n-1}$. For $g(x)<n-1$, it follows $g(z) \leq k$. 
2) For $z \in[k, n-1]$ we have $g(z) \geq k$.

Let $z \in[k, n-1]$. As $z \geq k$, we have $(z, y) \in \mathrm{R}_{0}^{k}$. Furthermore, $\mathrm{R}_{0}^{k} \in C \operatorname{Inv} g_{k}$, so $g \triangleright \mathrm{R}_{0}^{k}$. Thus, $(g(z), g(y)) \in \mathrm{R}_{0}^{k}$. As $g(y)>0$, it follows $g(z) \geq k$.

3) For all $z \in[0, k]$ we have $g(z) \geq z$.

Let $z \in[0, k]$. As $z \leq k$, the relation $\mathrm{R}_{0}^{z}$ belongs to $C \operatorname{Inv} g_{k}$. Clearly, the tuple $(z, y)$ belongs to $\mathrm{R}_{0}^{z}$ and hence $g \triangleright \mathrm{R}_{0}^{z}$. Thus, $(g(z), g(y)) \in \mathrm{R}_{0}^{z}$. For $g(y)>0$, it follows $g(z) \geq z$.

4) For all $z \in[k, n-1]$ we have $g(z) \leq z$.

Let $z \in[k, n-1]$. As $z \geq k$, the relation $\mathrm{R}_{z}^{n-1}$ belongs to $C$ Inv $g_{k}$. Clearly, the tuple $(x, z)$ belongs to $\mathrm{R}_{z}^{n-1}$ and hence $g \triangleright \mathrm{R}_{z}^{n-1}$. Thus, $(g(x), g(z)) \in \mathrm{R}_{z}^{n-1}$. For $g(x)<n-1$, it follows $g(z) \leq z$.

5) For all $z \in[0, k]$ it holds that $z \leq g(z) \leq k$, and for all $z \in[k, n-1]$ we have $k \leq g(z) \leq z$. In particular, $g(k)=k$.

This is a combination of 1), 2), 3), and 4).

6) For all $z \in[0, k)$ such that $g(z) \neq k$ we have $g(z)=z$.

Let $z \in[0, k)$ and $g(z) \neq k$, then by 5) we obtain $z \leq g(z)<k$. Obviously, $(z, z) \in \mathrm{R}_{z}^{k}$. Since $\mathrm{R}_{z}^{k} \in C \operatorname{Inv} g_{k}$, we get $g \triangleright \mathrm{R}_{z}^{k}$. Thus, $(g(z), g(z)) \in \mathrm{R}_{z}^{k}$. Since $g(z)<k$, it follows $g(z) \leq z$.

7) If $g(0)=k$, then $g(z) \leq k$ for all $z \in D$.

Let $g(0)=k$ and $z \in D$. Then it holds that $(k, 0, z) \in \mathrm{R}_{(0, k)}^{n-1}$, and $\mathrm{R}_{(0, k)}^{n-1}$

belongs to $C \operatorname{Inv} g_{k}$. Hence, we get $(k, k, g(z))=(g(k), g(0), g(z)) \in \mathrm{R}_{(0, k)}^{n-1}$.

Since $0<k<n-1$, it follows $g(z) \leq k$.

8) If there exists a $v \in[0, k)$ such that $g(v)=k$, then $g=c_{k}$.

Let $v \in[0, k)$ such that $g(v)=k$. Let $z \in D$, then $(z, v) \in \mathrm{R}_{v}^{k}$. Since $\mathrm{R}_{v}^{k} \in C \operatorname{Inv} g_{k}$, we receive $g \triangleright \mathrm{R}_{v}^{k}$, and hence $(g(z), k)=(g(z), g(v)) \in \mathrm{R}_{v}^{k}$. As $k>v$, we infer

$$
g(z) \geq k \text {. }
$$

Now 5) implies $g(z) \leq k$ for all $z \in[0, k]$. This yields the precondition of 7$)$. Hence, we can infer $g(z) \leq k$ for all $z \in D$. That together with (5.5), implies $g=c_{k}$.

9) For all $v \in[0, k]$ we have $g(v)=v$.

From 5) we infer $g(k)=k$. Since $g \neq c_{k}$, by 8) we infer that for all $v \in[0, k)$, $g(v) \neq k$. This yields the precondition of 6$)$. Hence, $g(v)=v$ for all $v \in[0, k]$.

10) For all $z \in(k, n-1]$ we have $g(z)=k$.

Since $g$ is not $i d_{D}$, there is some $c \in D$ such that $g(c) \neq c$. By 9$)$, we have $c \in(k, n-1]$ and by 5) we obtain $g(c)<c$. Let $z \in D$. The relation $\mathrm{R}_{k}^{c}$ belongs to $C \operatorname{Inv} g_{k}$, hence $g \triangleright \mathrm{R}_{k}^{c}$. Clearly, the tuple $(c, z)$ belongs to $\mathrm{R}_{k}^{c}$. 
Thus, $(g(c), g(z)) \in \mathrm{R}_{k}^{c}$. For $g(c)<c$, it follows $g(z) \leq k$ and by 5$) g(z) \geq k$ for all $z \in(k, n-1]$. From the last two inequalities, we get $g(z)=k$ for all $z \in(k, n-1]$.

Similarly, one can prove

5.5.7 Proposition. Let $k \in\{1, \ldots, n-2\}$ be fixed, and let $g_{k}$ be the unary operation given by $g_{k}^{\prime}(x)=\max \left(c_{k}(x), x\right)$ for $x \in D$. Then

$$
\left\langle g_{k}^{\prime}\right\rangle_{\mathcal{C O}(1)}=\left\{g_{k}^{\prime}, c_{k}, c_{0}, c_{n-1}, i d_{D}\right\}=\left\langle c_{0}, c_{n-1}, g_{k}^{\prime}\right\rangle_{O_{D}^{(1)}}
$$

From the restriction of the Galois connection End - Inv to the Galois connection End $-C \operatorname{Inv}$, we get $\operatorname{End} \operatorname{Inv}(F)=\langle F\rangle_{O_{D}^{(1)}} \subseteq\langle F\rangle_{\mathcal{C} O_{D}^{(1)}}$, for any set $F$ of operations on $D$. Hence, some essentially unary $C$-clones ( $C$-monoids) generated by some $F$ may contain more functions, which are not obtained in the usual form, that is, by composition of functions from $F$. One example is $\left\langle g_{k}\right\rangle_{\mathcal{C} O_{D}^{(1)}}$, see also Proposition 5.5.6. The question is to determine the new functions in

$$
\langle F\rangle_{\mathcal{C O}_{D}^{(1)}} \backslash\langle F\rangle_{O_{D}^{(1)}}
$$

in a constructive way that is essentially different from applying End $C$ Inv.

To end this chapter, let us summarize the results obtained in Proposition 5.5.6 and 5.5.7 in the following figure.

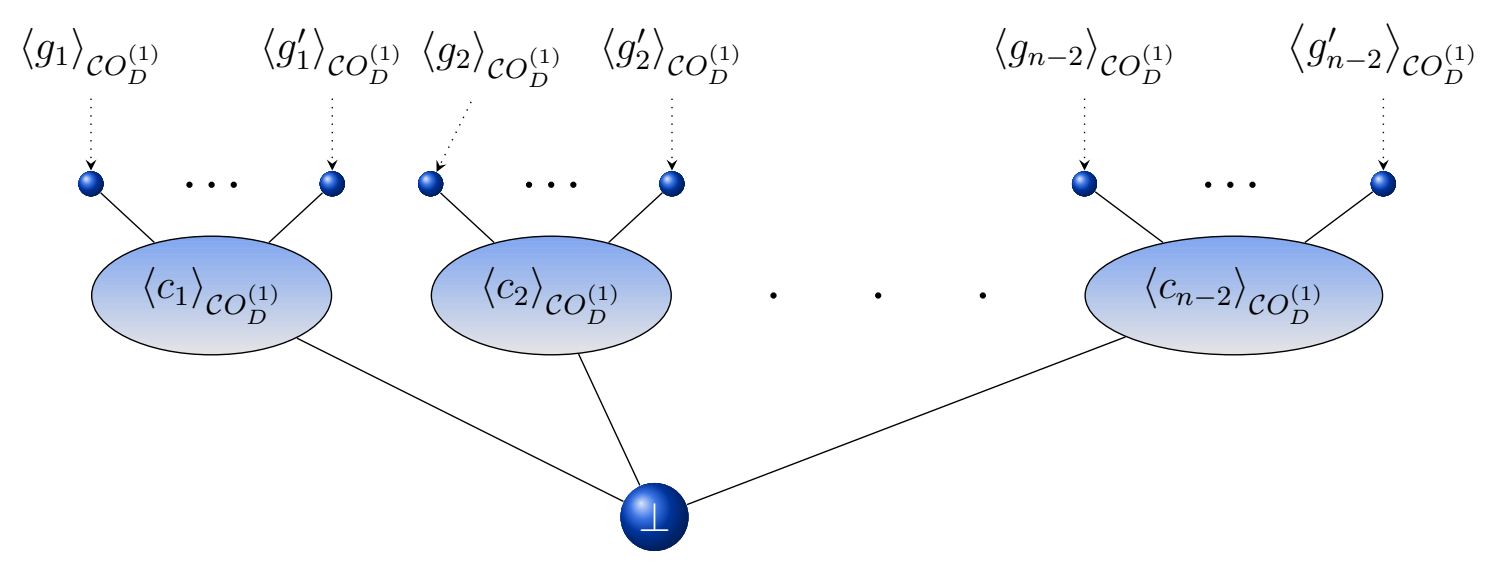





\section{Maximal $C$-clones}

\subsection{Description}

In this section we describe all co-atoms in the lattice of all $C$-clones for $|D| \geq 3$, these are formally defined as follows.

6.1.1 Definition. A $C$-clone $F$ is called maximal if $F \neq \mathrm{O}_{D}$ and between $F$ and $\mathrm{O}_{D}$ there are no more $C$-clones. That is, for every $C$-clone $F^{\prime}$ the condition $F \varsubsetneqq F^{\prime}$ implies $F^{\prime}=\mathrm{O}_{D}$.

Theorem 6.1 .5 below states that a $C$-clone is maximal if it can be determined by only one non-trivial clausal relation of arity two. To prove this theorem we need some auxiliary statements such as Lemmata 6.1.3, 6.1.4 and Remark 6.1.2. The latter states that $C$-clones of the form Pol R ${ }_{b}^{a}$, where $a \in D \backslash\{0\}$ and $b \in D \backslash\{n-1\}$, are candidates for maximal $C$-clones.

6.1.2 Remark. Let $a \in D \backslash\{0\}$ and $b \in D \backslash\{n-1\}$. Then

$$
\operatorname{Pol} \mathrm{R}_{b}^{a} \varsubsetneqq \mathrm{O}_{D}
$$

Proof. Assume $\mathrm{Pol} \mathrm{R}_{b}^{a}=\mathrm{O}_{D}$. Hence, we get $\mathrm{O}_{D}^{(1)} \cap \mathrm{Pol}_{b}^{a}=\mathrm{O}_{D}^{(1)}$, i.e. End $\mathrm{R}_{b}^{a}=\mathrm{O}_{D}^{(1)}$. The latter is a contradiction to End $\mathrm{R}_{b}^{a} \varsubsetneqq \mathrm{O}_{D}^{(1)}$ (cf. Remark 5.3.2)

6.1.3 Lemma. Let $f \in \mathrm{O}_{D}, a_{1}, \ldots, a_{p} \in D \backslash\{0\}$ and $b_{1}, \ldots, b_{q} \in D \backslash\{n-1\}$. Then we have

$$
f \triangleright \mathrm{R}_{b_{1}, \ldots, b_{q}}^{a_{1}, \ldots, a_{p}} \Rightarrow f \triangleright \mathrm{R}_{\max \left\{b_{1}, \ldots, b_{q}\right\}}^{\min \left\{a_{1}, \ldots, a_{p}\right\}} .
$$

Proof. Let $k \in \mathbb{N}_{+}$and $f$ be a $k$-ary function on $D$ such that $f \triangleright \mathrm{R}_{b_{1}, \ldots, b_{q}}^{a_{1}, \ldots, a_{p}}$. We have to show that $f$ preserves $\mathrm{R}_{\max \left\{b_{1}, \ldots, b_{q}\right\}}^{\min \left\{a_{1}, \ldots, a_{p}\right\}}$. For $l \in\{1, \ldots, k\}$ we consider $\mathbf{r}_{l}:=\left(x_{l}, y_{l}\right)$ to be any tuples that belong to $\mathrm{R}_{\max \left\{b_{1}, \ldots, b_{q}\right\}}^{\min \left\{a_{1}, \ldots, a_{p}\right\}}$. The latter implies

$$
\begin{aligned}
x_{l} \geq \min \left\{a_{1}, \ldots, a_{p}\right\} \vee y_{l} & \leq \max \left\{b_{1}, \ldots, b_{q}\right\} \\
\Longleftrightarrow x_{l} \geq a_{1} \vee \ldots \vee x_{l} \geq a_{p} \vee y_{l} & \leq b_{1} \vee \ldots \vee y_{l} \leq b_{q}
\end{aligned}
$$

Because of the expression above, the tuples $\left(x_{l}, \ldots, x_{l}, y_{l}, \ldots, y_{l}\right)$ belong to $\mathrm{R}_{b_{1}, \ldots, b_{q}}^{a_{1}, \ldots, a_{p}}$, where $l \in\{1, \ldots, k\}$. Furthermore, since $f \triangleright \mathrm{R}_{b_{1}, \ldots, b_{q}}^{a_{1}, \ldots, a_{p}}$, we obtain

$$
\begin{aligned}
& f\left(x_{1}, \ldots, x_{k}\right) \geq a_{1} \vee f\left(x_{1}, \ldots, x_{k}\right) \geq a_{2} \ldots \vee f\left(x_{1}, \ldots, x_{k}\right) \geq a_{p} \vee \\
& f\left(y_{1}, \ldots, y_{k}\right) \leq b_{1} \vee f\left(y_{1}, \ldots, y_{k}\right) \leq b_{2} \vee \ldots \vee f\left(y_{1}, \ldots, y_{k}\right) \leq b_{q} \\
\Longleftrightarrow & f\left(x_{1}, \ldots, x_{k}\right) \geq \min \left\{a_{1}, \ldots, a_{p}\right\} \vee f\left(y_{1}, \ldots, y_{k}\right) \leq \max \left\{b_{1}, \ldots, b_{q}\right\} .
\end{aligned}
$$

Thus, $f\left[\mathbf{r}_{1}, \ldots, \mathbf{r}_{k}\right]=\left(f\left(x_{1}, \ldots, x_{k}\right), f\left(y_{1}, \ldots, y_{k}\right)\right) \in \mathrm{R}_{\max \left\{b_{1}, \ldots, b_{q}\right\}}^{\min \left\{a_{1}, \ldots, a_{p}\right\}}$. 
We can also prove that any two $C$-monoids, $\operatorname{Pol} \mathrm{R}_{b_{1}}^{a_{1}}$ and $\mathrm{Pol}_{b_{2}}^{a_{2}}$, where

$$
\left(a_{1}, b_{1}\right) \neq\left(a_{2}, b_{2}\right)
$$

and $\left(a_{1}, b_{1}\right),\left(a_{2}, b_{2}\right) \in(D \backslash\{0\}) \times(D \backslash\{n-1\})$, are incomparable.

6.1.4 Lemma. Let $a_{1}, a_{2} \in D \backslash\{0\}, b_{1}, b_{2} \in D \backslash\{n-1\}$. If $\left(a_{1}, b_{1}\right) \neq\left(a_{2}, b_{2}\right)$, then we have

$$
\text { Pol } \mathrm{R}_{b_{1}}^{a_{1}} \nsubseteq \mathrm{Pol}_{b_{2}}^{a_{2}} \quad \text { and } \quad \operatorname{Pol}_{b_{2}}^{a_{2}} \nsubseteq \mathrm{Pol}_{b_{1}}^{a_{1}}
$$

Proof. Let Pol $\mathrm{R}_{b_{1}}^{a_{1}}$ and Pol $\mathrm{R}_{b_{2}}^{a_{2}}$, where $\left(a_{1}, b_{1}\right) \neq\left(a_{2}, b_{2}\right)$. Let us assume

$$
\text { Pol R } b_{1} \subseteq \text { Pol R } b_{b_{2}}^{a_{2}} \quad \text { or } \quad \text { Pol R } \text { R }_{b_{2}}^{a_{2}} \subseteq \text { Pol R } \mathrm{R}_{b_{1}}^{a_{1}}
$$

If Pol $\mathrm{R}_{b_{1}}^{a_{1}} \subseteq$ Pol R $\mathrm{R}_{b_{2}}^{a_{2}}$, then End $\mathrm{R}_{b_{1}}^{a_{1}}=\mathrm{O}_{D}^{(1)} \cap \operatorname{Pol} \mathrm{R}_{b_{1}}^{a_{1}} \subseteq \mathrm{O}_{D}^{(1)} \cap \mathrm{Pol} \mathrm{R}_{b_{2}}^{a_{2}}=$ End $\mathrm{R}_{b_{2}}^{a_{2}}$. The latter is a contradiction to End $\mathrm{R}_{b_{1}}^{a_{1}} \nsubseteq$ End $\mathrm{R}_{b_{2}}^{a_{2}}$. (cf. Lemma 5.3.4)

If $\mathrm{Pol} \mathrm{R}_{b_{2}}^{a_{2}} \subseteq$ Pol $\mathrm{R}_{b_{1}}^{a_{1}}$, then End $\mathrm{R}_{b_{2}}^{a_{2}}=\mathrm{O}_{D}^{(1)} \cap \mathrm{Pol} \mathrm{R}_{b_{2}}^{a_{2}} \subseteq \mathrm{O}_{D}^{(1)} \cap \mathrm{Pol}_{b_{1}}^{a_{1}}=$ End $\mathrm{R}_{b_{1}}^{a_{1}}$, which is a contradiction to End $\mathrm{R}_{b_{2}}^{a_{2}} \nsubseteq$ End $\mathrm{R}_{b_{1}}^{a_{1}}$.

Below we characterise all maximal $C$-clones.

6.1.5 Theorem. Let $M \subseteq \mathrm{O}_{D}$ be a $C$-clone. $M$ is maximal if and only if there are elements $a \in D \backslash\{0\}$ and $b \in D \backslash\{n-1\}$ such that

$$
M=\operatorname{Pol} \mathrm{R}_{b}^{a} .
$$

Proof. " $\Rightarrow$ :" Let $M \subseteq \mathrm{O}_{D}$ be a maximal $C$-clone. Then there exists a relation $\varrho \in C \mathrm{R}_{D}^{*}$ such that $M=\mathrm{Pol} \varrho$. This means there are elements $a_{1} \ldots a_{p} \in D \backslash\{0\}$ and $b_{1}, \ldots, b_{q} \in D \backslash\{n-1\}$ such that $M=\operatorname{Pol~R}_{b_{1}, \ldots, b_{q}}^{a_{1}, \ldots, a_{p}}$. From 6.1.3 and 6.1.2, we have

$$
M=\mathrm{PolR}_{b_{1}, \ldots, b_{q}}^{a_{1}, \ldots, a_{p}} \stackrel{6.1 .3}{\subseteq} \operatorname{Pol} \mathrm{R}_{\max \left\{b_{1}, \ldots, b_{q}\right\}}^{\min \left\{a_{1}, \ldots, a_{p}\right\}} \underset{f}{\stackrel{6.1 .2}{\subsetneq}} \mathrm{O}_{D} .
$$

Since $M$ is maximal, we have $M=\operatorname{Pol} \mathrm{R}_{\max \left\{b_{1}, \ldots, b_{q}\right\}}^{\min \left\{a_{1}, \ldots, a_{p}\right\}}$.

" $\Leftarrow$ :" Let Pol $\mathrm{R}_{b}^{a}$ be a $C$-clone, where $a \in D \backslash\{0\}$ and $b \in D \backslash\{n-1\}$. To prove that $\mathrm{Pol} \mathrm{R}_{b}^{a}$ is maximal we consider a $C$-clone $C$ such that $\mathrm{Pol}_{b}^{a} \varsubsetneqq C$ and we have to show that $C=\mathrm{O}_{D}$.

Since $\operatorname{PolR}_{b}^{a} \varsubsetneqq C$, there is some $f \in C$ and $f \notin \operatorname{PolR}_{b}^{a}$. As $f \in C$ and $\operatorname{Pol} \mathrm{R}_{b}^{a} \varsubsetneqq C$, we get $\{f\} \cup \operatorname{Pol} \mathrm{R}_{b}^{a} \subseteq C$. Hence,

$$
\operatorname{Pol} C \operatorname{Inv}\left(\{f\} \cup \operatorname{Pol} \mathrm{R}_{b}^{a}\right)=\left\langle\{f\} \cup \operatorname{Pol} \mathrm{R}_{b}^{a}\right\rangle_{\mathcal{C}} \subseteq\langle C\rangle_{\mathcal{C}}=C \subseteq \mathrm{O}_{D} .
$$

We consider two cases:

- If Pol $C \operatorname{Inv}\left(\{f\} \cup \operatorname{Pol} \mathrm{R}_{b}^{a}\right)=\mathrm{O}_{D}$, then from (6.1) we infer $C=\mathrm{O}_{D}$. Thus, $\mathrm{Pol} \mathrm{R}_{b}^{a}$ is maximal. 
- If Pol $C \operatorname{Inv}\left(\{f\} \cup \operatorname{Pol} \mathrm{R}_{b}^{a}\right) \varsubsetneqq \mathrm{O}_{D}$, then

$$
C \operatorname{Inv}\left(\{f\} \cup \operatorname{Pol} \mathrm{R}_{b}^{a}\right) \supsetneqq C \operatorname{Inv} \mathrm{O}_{D} \stackrel{3.4 .3}{=}\left\{D^{p+q} \mid p, q \in \mathbb{N}_{+}\right\} \text {, }
$$

and hence there is some relation $\varrho$ belonging to $C \operatorname{Inv}\left(\{f\} \cup \operatorname{Pol} \mathrm{R}_{b}^{a}\right)$, which is non-trivial. Thus, $\varrho=\mathrm{R}_{\mathbf{b}^{\prime}}^{\mathbf{a}^{\prime}}$, where $\mathbf{a}^{\prime} \in(D \backslash\{0\})^{p}$ and $\mathbf{b}^{\prime} \in(D \backslash\{n-1\})^{q}$.

Furthermore,

Pol $\mathrm{R}_{\max \left\{b_{1}^{\prime}, \ldots, b_{q}^{\prime}\right\}}^{\min \left\{a_{1}^{\prime}, \ldots, a_{p}^{\prime}\right\}} \stackrel{6.1 .3}{\supseteq} \operatorname{Pol} \varrho \supseteq \operatorname{Pol} C \operatorname{Inv}\left(\{f\} \cup \operatorname{Pol} \mathrm{R}_{b}^{a}\right) \supseteq \operatorname{Pol} C \operatorname{Inv}\left(\operatorname{Pol} \mathrm{R}_{b}^{a}\right)=\operatorname{Pol} \mathrm{R}_{b}^{a}$

Hence, $f \in \operatorname{Pol}_{\max \left\{b_{1}^{\prime}, \ldots, b_{q}^{\prime}\right\}}^{\min \left\{a_{1}^{\prime}, \ldots, a_{p}^{\prime}\right\}} \supseteq \operatorname{Pol} \mathrm{R}_{b}^{a}$. Because of 6.1 .4 , we have $a=\min \left\{a_{1}^{\prime}, \ldots, a_{p}^{\prime}\right\}$ and $b=\max \left\{b_{1}^{\prime}, \ldots, b_{q}^{\prime}\right\}$. Thus, $\operatorname{Pol}_{\max \left\{b_{1}^{\prime}, \ldots, b_{q}^{\prime}\right\}}^{\min \left\{a_{1}^{\prime}, \ldots, a_{p}^{\prime}\right\}}=\operatorname{PolR}_{b}^{a}$ and hence $f \in \operatorname{PolR}_{b}^{a}$, which is a contradiction to $f \notin \operatorname{Pol} \mathrm{R}_{b}^{a}$.

Due to 6.1 .5 and 3.4.4, we get

$$
\begin{gathered}
\langle h\rangle_{\mathcal{C}}=\operatorname{Pol}\left\{\mathrm{R}_{b}^{a} \mid a \in D \backslash\{0\}, b \in D \backslash\{n-1\}\right\} \\
\stackrel{\text { 6.1.5 }}{=} \bigcap\{M \mid M \text { maximal } C \text {-clone }\} .
\end{gathered}
$$

Note that one non-trivial clausal relation of arity two can be used to describe both a co-atom in the lattice of $C$-clones and a co-atom in the lattice of $C$-monoids, in contrast to the normal maximal clones where this does not happen. In fact, there are also $(n-1)^{2}$ maximal $C$-clones on an $n$-element set $D$.

\subsection{C-complete operations}

We return now to unary operations, referring back to Figure 5.3, we can see that there is a considerable number of unary operations, located at the top in this lattice. In this section these sets of operations are characterised.

6.2.1 Definition. A set $F \subseteq \mathrm{O}_{D}^{(1)}$ of unary operations on $D$ is $C$-complete if

$$
\langle F\rangle_{\mathcal{C O}}(1)=\mathrm{O}_{D}^{(1)}
$$

Theorem 6.2.3 below states that the set containing only one unary operation is $C$-complete if it does not preserve all non-trivial clausal relations of arity two. To prove this theorem we need the following statement.

6.2.2 Lemma. Let $F \subseteq \mathrm{O}_{D}^{(1)}$. Then $F$ is $C$-complete if and only if $F$ is not contained in any maximal $C$-monoid. 
Proof. " $\Rightarrow:$ Let $F \subseteq \mathrm{O}_{D}^{(1)}$ be a $C$-complete set of unary operations. Assume $F$ is contained in $M$ for some maximal $C$-monoid $M$. The latter implies

$$
\mathrm{O}_{D}^{(1)}=\langle F\rangle_{\mathcal{C O}}(1) \subseteq\langle M\rangle_{\mathcal{C} O_{D}^{(1)}}=M \varsubsetneqq \mathrm{O}_{D}^{(1)}
$$

which is a contradiction.

"६:" In order to show $\langle F\rangle_{\mathcal{C} O_{D}^{(1)}}=\mathrm{O}_{D}^{(1)}$, we assume $\langle F\rangle_{\mathcal{C O}} O_{D}^{(1)} \varsubsetneqq \mathrm{O}_{D}^{(1)}$. Since $\mathrm{O}_{D}^{(1)}$ is finite, the lattice of all $C$-monoids is also finite, thus there exists a maximal $C$-monoid $M$ which contains $\langle F\rangle_{\mathcal{C} O_{D}^{(1)}}$, hence $F \subseteq\langle F\rangle_{\mathcal{C} O_{D}^{(1)}} \subseteq M$, which is also a contradiction.

6.2.3 Theorem. Let $g \in \mathrm{O}_{D}^{(1)}$ be a unary operation, $\{g\}$ is $C$-complete if and only if $g \not \mathrm{R}_{b}^{a}$ for all $(a, b) \in D \backslash\{0\} \times D \backslash\{n-1\}$.

Proof. Let $\{g\} \in \mathrm{O}_{D}^{(1)}$ be $C$-complete. By Theorem 5.3.5 each maximal $C$-monoid $M$ is of the form $M=$ End $\mathrm{R}_{b}^{a}$ where $a \in D \backslash\{0\}$ and $b \in D \backslash\{n-1\}$. Thus, $\{g\} \nsubseteq M \Longleftrightarrow g \not \mathrm{R}_{b}^{a}$ for all $(a, b) \in D \backslash\{0\} \times D \backslash\{n-1\}$. Therefore, the theorem follows from 6.2.2.

In the following if we speak of a $C$-complete unary operation, we implicitly mean that the singleton set containing the unary operation is $C$-complete.

Due to Theorem 6.2.3 for $g \in \mathrm{O}_{D}^{(1)}$, deciding if $\{g\}$ is $C$-complete is equivalent to the task of finding for all $(a, b) \in D \backslash\{0\} \times D \backslash\{n-1\}$ a tuple $\mathbf{r}$ belonging to $\mathrm{R}_{b}^{a}$ such that $g[\mathbf{r}] \notin \mathrm{R}_{b}^{a}$.

Observe that the tuples $\mathbf{t}_{d}:=(n-1, d)$ and $\mathbf{t}^{\prime}{ }_{d}:=(d, 0)$, where $d \in D$, belong to $\mathrm{R}_{b}^{a}$ for all $(a, b) \in D \backslash\{0\} \times D \backslash\{n-1\}$. However, the tuple $(0, n-1)$ does not belong to $\mathrm{R}_{b}^{a}$.

A form to find conditions under which some unary operations are $C$-complete, is to look for unary operations $g_{d}$ on $D$ such that $g_{d}\left[\mathbf{t}_{d}\right]=(0, n-1)$, for some $d \in\{0,1, \ldots, n-1\}$. The latter implies

$$
g_{d}(n-1)=0 \text { and } g_{d}(d)=n-1 \text { and } d \neq n-1 .
$$

From the previous observation and Theorem 6.2.3, we infer that for fixed $d$ belonging to $\{0, \ldots, n-2\}$, the sets $\left\{g_{d}\right\}$, where $g_{d}$ satisfies

$$
g_{d}(x):= \begin{cases}n-1 & \text { if } x=d \\ 0 & \text { if } x=n-1\end{cases}
$$

are $C$-complete.

Similarly, if we consider unary operations $\widehat{g_{d}}$ on $D$ such that ${\widehat{g_{d}}}_{d}\left[\mathbf{t}_{d}^{\prime}\right]=(0, n-1)$, i.e. such that $\widehat{g_{d}}(d)=0$ and $\widehat{g_{d}}(0)=n-1$ and $d \neq 0$. For fixed $d \in\{1, \ldots, n-1\}$ the sets $\left\{\widehat{g_{d}}\right\}$, where $\widehat{g_{d}}$ satisfies

$$
\widehat{g_{d}}(x):= \begin{cases}0 & \text { if } x=d \\ n-1 & \text { if } x=0\end{cases}
$$


are $C$-complete.

Below the number of unary operations that satisfy $\left(I_{d}\right)$ or $\left(I_{d}^{\prime}\right)$ are counted. Since $\left(I_{d}\right)$ for $d=0$ is identical to $\left(I_{d}^{\prime}\right)$ for $d=n-1$, we count unary operations that satisfy $\left(I_{d}^{\prime}\right)$ for $0<d<n-1$, or $\left(I_{d}\right)$ for $0 \leq d<n-1$.

- For $D=\{0,1\}$ there is only one operation $g_{0}$ satisfying $\left(I_{d}\right)$. It is equal to $\neg$ the negation operation.

- For $D=\{0,1,2\}$, there are seven unary operations that are $C$-complete (see Fig.5.3), all of them are listed in the following table, its last row is labelled by the number of operations that satisfy $\left(I_{d}\right)$ for $d \in\{0,1\}$ or $\left(I_{d}^{\prime}\right)$ for $d=1$. For example in the last row, second column, $3=\mid\left\{f \in \mathrm{O}_{D}^{(1)} \mid f\right.$ satisfy $\left.I_{0}\right\} \mid$.

\begin{tabular}{|c|c|c|c||c|}
\hline$x$ & $g_{0}(x)$ & $g_{1}(x)$ & $\widehat{g}_{1}(x)$ & $\sum$ \\
\hline \hline 0 & $\mathbf{2}$ & 01 & $\mathbf{2}$ & \\
\hline 1 & 012 & $\mathbf{2}$ & $\mathbf{0}$ & \\
\hline 2 & $\mathbf{0}$ & $\mathbf{0}$ & 12 & \\
\hline & 3 & 2 & 2 & $3+2+2=7$ \\
\hline
\end{tabular}

- For $D=\{0,1,2,3\}$, there are 58 operations of the form $\left(I_{d}\right)$ for $d \in\{0,1,2\}$ or $\left(I_{d}^{\prime}\right)$ for $d \in\{1,2\}$, calculated as follows.

\begin{tabular}{|c|c|c|c|c|c|c|}
\hline$x$ & $g_{0}(x)$ & $g_{1}(x)$ & $g_{2}(x)$ & $\widehat{g}_{1}(x)$ & $\widehat{g}_{2}(x)$ & $\sum$ \\
\hline \hline 0 & $\mathbf{3}$ & 012 & 012 & $\mathbf{3}$ & $\mathbf{3}$ & \\
\hline 1 & 0123 & $\mathbf{3}$ & 012 & $\mathbf{0}$ & 123 & \\
\hline 2 & 0123 & 0123 & $\mathbf{3}$ & 0123 & $\mathbf{0}$ & \\
\hline 3 & $\mathbf{0}$ & $\mathbf{0}$ & $\mathbf{0}$ & 123 & 123 & \\
\hline & $4^{2}$ & $3 \times 4$ & $3^{2}$ & $4 \times 3$ & $3^{2}$ & $=58$ \\
\hline
\end{tabular}

- For $|D| \geq 3$, the number of operations of the form $\left(I_{d}\right)$ for $0 \leq d<n-1$ or of the form $\left(I_{d}^{\prime}\right)$ for $0<d<n-1$ is:

$$
\begin{aligned}
& \mid\left.\left\{f \in \mathrm{O}_{D}^{(1)} \mid f \text { satisfy }\left(I_{d}\right) ; 0 \leq d<n-1\right\}\left|=\sum_{i=0}^{n-2}\right| D\right|^{(|D|-2)-i} *(|D|-1)^{i}, \\
& \mid\left.\left\{f \in \mathrm{O}_{D}^{(1)} \mid f \text { satisfy }\left(I_{d}^{\prime}\right) ; 0<d<n-1\right\}\left|=\sum_{i=0}^{n-3}\right| D\right|^{(|D|-3)-i} *(|D|-1)^{i+1} .
\end{aligned}
$$

Contrary to $D=\{0,1,2\}$, on $D=\{0,1,2,3\}$, there are more unary operations that are $C$-complete, but they do neither satisfy $\left(I_{d}\right)$ for $0 \leq d<3$ nor $\left(I_{d}^{\prime}\right)$ for $0<d<3$. For example, the unary operation $g^{*}$ such that

$$
g^{*}(0)=1, g^{*}(1)=3, g^{*}(2)=0, g^{*}(3)=2 .
$$

does neither satisfy $\left(I_{d}\right)$ where $0 \leq d<3$ nor $\left(I_{d}^{\prime}\right)$ where $0<d<3$. However, it is $C$-complete, as it is proved in the following remark. 
6.2.4 Remark. Let $D=\{0,1,2,3\}$ and $g^{*}$ be defined as above. Then $\left\{g^{*}\right\}$ is C-complete.

Proof. Let $M:=\left\{\mathrm{R}_{b}^{a} \mid a \in\{1,2,3\}, b \in\{0,1,2\}\right\}$. Due to Theorem 6.2.3, it is enough to prove that $g^{*}$ does not preserve $\varrho$ for any $\varrho \in M$. Consider $M=M_{1} \cup M_{2}$, where

$$
M_{1}=\left\{\mathrm{R}_{0}^{a} \mid a \in\{1,2,3\}\right\} \text { and } M_{2}=\left\{\mathrm{R}_{b}^{a} \mid a \in\{1,2,3\}, b \in\{1,2\}\right\}
$$

and let $\varrho \in M$. We consider two cases:

If $\varrho \in M_{1}$, then $\varrho=\mathrm{R}_{0}^{a}$, where $a \in\{1,2,3\}$. As the tuple $(2,0)$ belongs to $\varrho$, and $\left(g^{*}(2), g^{*}(0)\right)=(0,1)$ does not, we get $g^{*} \not \varrho$.

If $\varrho \in M_{2}$, then $\varrho=\mathrm{R}_{b}^{a}$, where $a \in\{1,2,3\}$ and $b \in\{1,2\}$. Since the tuple $(2,1)$ belongs to $\varrho$, and $\left(g^{*}(2), g^{*}(1)\right)=(0,3)$ does not belong to $\varrho$, we obtain $g^{*} \not \varrho$.

More conditions under which unary operations on $D$ are $C$-complete, for $|D| \geq 4$, are established below.

Let $M:=\left\{\mathrm{R}_{b}^{a} \mid a \in D \backslash\{0\}, b \in D \backslash\{n-1\}\right\}$ be the set of all non-trivial clausal relations of arity two on $D$. For fixed $e_{1} \in\{1, \ldots, n-2\}$ we divide $M$ in two disjoint sets $M=M_{1} \dot{\cup} M_{2}$, where

$$
M_{1}=\left\{\mathrm{R}_{b}^{a} \mid b<e_{1}, a \in D \backslash\{0\}\right\} \text { and } M_{2}=\left\{\mathrm{R}_{b}^{a} \mid n-1>b \geq e_{1}, a \in D \backslash\{0\}\right\}
$$

Due to Theorem 6.2.3 a set $\{f\}$ is $C$-complete for $f \in \mathrm{O}_{D}^{(1)}$, if one finds a tuple $\mathbf{r}$ belonging to $\varrho$ for all $\varrho \in M=M_{1} \dot{\cup} M_{2}$ such that $f[\mathbf{r}] \notin \varrho$.

Let $d \in D$ be fixed. The tuple $(d, 0)$ belongs to $\varrho$ for all $\varrho \in M_{1}$, and the tuple $\left(d, e_{1}\right)$ belongs to $\varrho$ for all $\varrho \in M_{2}$.

The equality $f\left[\left(d, e_{1}\right)\right]=(0, n-1)$ is equivalent to

$$
f(d)=0 \text { and } f\left(e_{1}\right)=n-1 \text { and } d \neq e_{1} .
$$

If this condition is fulfilled, then $f \not \varrho$ for all $\varrho \in M_{2}$. Furthermore, the fact $f[(d, 0)] \in\{0\} \times\left\{e_{1}, e_{1}+1, \ldots, n-1\right\}$ is equivalent to

$$
f(d)=0 \text { and } f(0) \in\left[e_{1}, n-1\right] \text { and } d \neq 0 .
$$

In this case $f$ preserves no $\varrho \in M_{1}$. From the latter and Theorem 6.2.3, we infer.

If there are elements $e_{1} \in\{1, \ldots, n-2\}$ and $d \in D$, such that $0 \neq d \neq e_{1}$, then the sets $\{f\}$ on $D$, where $f$ satisfies

1) $f(d)=0$

2) $f\left(e_{1}\right)=n-1$,

3) $f(0) \geq e_{1}$, 
are $C$-complete.

Note that the unary operation $g^{*}$ (see 6.2.4) satisfies the latter conditions, for $e_{1}=1$ and $d=2$.

Similarly, for fixed $e_{1} \in\{1, \ldots, n-2\}$ we divide $M$ as $M=M_{1} \dot{\cup} M_{2}$, where

$M_{1}=\left\{\mathrm{R}_{b}^{a} \mid a \geq e_{1}, b \in D \backslash\{n-1\}\right\}$ and $M_{2}=\left\{\mathrm{R}_{b}^{a} \mid 0<a<e_{1}, b \in D \backslash\{n-1\}\right\}$.

Let $d \in D$ be fixed. The tuple $(n-1, d)$ belongs to $\varrho$ for all $\varrho \in M_{1}$, and the tuple $\left(e_{1}, d\right)$ belongs to $\varrho$ for all $\varrho \in M_{2}$.

The fact $f[(n-1, d)] \in\left\{e_{1}-1, e_{1}-2, \ldots, 0\right\} \times\{n-1\}$ is equivalent to

$$
f(n-1) \in\left[0, e_{1}-1\right] \text { and } f(d)=n-1 \text { and } d \neq n-1 .
$$

In this case $f$ preserves no $\varrho \in M_{1}$. Furthermore, the equality $f\left[\left(e_{1}, d\right)\right]=(0, n-1)$, is equivalent to

$$
f\left(e_{1}\right)=0 \text { and } f(d)=n-1 \text { and } d \neq e_{1} .
$$

If this condition is fulfilled, then $f \not \varrho$ for all $\varrho \in M_{2}$. Thus, we get the following assertion:

If there are elements $e_{1} \in\{1, \ldots, n-2\}$ and $d \in D$ such that $n-1 \neq d \neq e_{1}$, then the sets of unary operations $\{f\}$, where $f$ satisfies

1') $f(d)=n-1$,

2') $f\left(e_{1}\right)=0$,

3') $f(n-1) \leq e_{1}-1$,

are $C$-complete.

On $D=\{0,1,2,3,4\}$ there are still more unary operations that are $C$-complete but they do neither satisfy the conditions 1),2),3) nor the conditions $\left.\left.1^{\prime}\right), 2^{\prime}\right), 3^{\prime}$ ). Furthermore, they are neither of the form $\left(I_{d}\right)$ nor of the form $\left(I_{d}^{\prime}\right)$. For example ${ }^{1}$ the function $f_{1104}$ given by

$$
f_{1104}(0)=1, f_{1104}(1)=3, f_{1104}(2)=4, f_{1104}(3)=0, f_{1104}(4)=4 \text {. }
$$

is one of them.

More conditions under which unary operations are $C$-complete can be found as follows. We divide the set $M$ in three disjoint sets $M=M_{1} \dot{\cup} M_{2} \dot{\cup} M_{3}$, where

$$
\begin{aligned}
& M_{1}=\left\{\mathrm{R}_{b}^{a} \mid b<e_{1}, a \in D \backslash\{0\}\right\}, \\
& M_{2}=\left\{\mathrm{R}_{b}^{a} \mid e_{1} \leq b<e_{2}, a \in D \backslash\{0\}\right\}, \\
& M_{3}=\left\{\mathrm{R}_{b}^{a} \mid e_{2} \leq b<n-1, a \in D \backslash\{0\}\right\},
\end{aligned}
$$

\footnotetext{
${ }^{1}$ The index $k$ corresponding to this function is just the interpretation of $f_{k}(0) f_{k}(1) \ldots f_{k}(4)$ as a 5-adic number: $k=\sum_{i=0}^{4} f_{k}(i) 5^{4-i}$.
} 
for certain $e_{1}, e_{2} \in\{1, \ldots, n-2\}$ satisfying $e_{1} \neq e_{2}$.

We look for tuples belonging to $\varrho$ for all $\varrho \in M=M_{1} \cup M_{2} \dot{\cup} M_{3}$. Let $d \in D$ be fixed. The tuple $(d, 0)$ belongs to $\varrho$ for all $\varrho \in M_{1}$, the tuple $\left(d, e_{1}\right)$ belongs to $\varrho$ for all $\varrho \in M_{2}$, and the tuple $\left(d, e_{2}\right)$ belongs to $\varrho$ for all $\varrho \in M_{3}$. Finally, we look for conditions, under which unary operations $f$ do not preserve any for $\varrho \in M$.

- The equality $f\left[\left(d, e_{2}\right)\right]=(0, n-1)$ is equivalent to

$$
f(d)=0 \text { and } f\left(e_{2}\right)=n-1 \text { and } d \neq e_{2} .
$$

If this condition is fulfilled, then $f \not \varrho$ for all $\varrho \in M_{3}$.

- The fact $f\left[\left(d, e_{1}\right)\right] \in\{0\} \times\left\{e_{2}, e_{2}+1, \ldots, n-1\right\}$, is equivalent to

$$
f(d)=0 \text { and } f\left(e_{1}\right) \in\left[e_{2}, n-1\right] \text { and } d \neq e_{1} .
$$

In this case $f \not \varrho$ for all $\varrho \in M_{2}$,

- and $f[(d, 0)] \in\{0\} \times\left\{e_{1}, e_{1}+1, \ldots, n-1\right\}$, is equivalent to

$$
f(d)=0 \text { and } f(0) \in\left[e_{1}, n-1\right] \text { and } d \neq 0 .
$$

In this case $f$ preserves no $\varrho \in M_{1}$.

From the previous conditions and Theorem 6.2.3 we infer:

If there are elements $e_{1}, e_{2} \in\{1, \ldots, n-2\}$ and $d \in D$ such that

$$
0 \neq d \neq e_{1} \neq e_{2} \neq d,
$$

then sets of unary operations $\{f\}$, where $f$ satisfies

i) $f(d)=0$,

ii) $f(0) \geq e_{1}$,

iii) $f\left(e_{1}\right) \geq e_{2}$,

iv) $f\left(e_{2}\right)=n-1$,

are $C$-complete.

Note that the unary operation $f_{1104}$ defined above satisfies the latter conditions for $e_{1}=1, e_{2}=2$ and $d=3$.

Similarly, for $e_{1}, e_{2} \in\{1, \ldots, n-2\}$, where $e_{1} \neq e_{2}$, we divide $M=M_{1} \dot{\cup} M_{2} \dot{\cup} M_{3}$, where

$$
\begin{aligned}
& M_{1}=\left\{\mathrm{R}_{b}^{a} \mid a \geq e_{1}, b \in D \backslash\{n-1\}\right\}, \\
& M_{2}=\left\{\mathrm{R}_{b}^{a} \mid e_{2} \leq a<e_{1}, b \in D \backslash\{n-1\}\right\}, \\
& M_{3}=\left\{\mathrm{R}_{b}^{a} \mid 0<a<e_{2}, b \in D \backslash\{n-1\}\right\} .
\end{aligned}
$$

Finally, we get: If there are elements $e_{1}, e_{2} \in\{1, \ldots, n-2\}$ and $d \in D$ such that $0 \neq d \neq e_{1} \neq e_{2} \neq d$, then sets of unary operations $\{f\}$, where $f$ satisfies 
i') $f(d)=n-1$

ii') $f(n-1)<e_{1}$,

iii') $f\left(e_{1}\right)<e_{2}$,

iv') $f\left(e_{2}\right)=0$,

are $C$-complete.

Further conditions under which the sets $\{f\}$ for $f \in \mathrm{O}_{D}^{(1)}$ are $C$-complete, can be found if we divide $M$ in more disjoint subsets. Possibly the conditions obtained in this way are enough to determine when a set containing only one unary operation on $D$ is $C$-complete. We leave it as an open problem, to solve the following question.

Given an arbitrary unary operation $f$ on a finite set $D$, decide if the set $\{f\}$ is $C$-complete or not. 



\section{Problems for future research}

In this chapter some open problems are presented that emerged while working on this dissertation, but, unfortunately, could not be solved so far.

The first questions are closely related to cardinalities of the $C$-clone lattice $\mathcal{C} \mathcal{L}_{D}$ for a finite set $|D| \geq 3$.

P1. In Section 3.5, we showed that for $|D| \geq 3$ there exist infinitely many $C$-clones. However, how many $C$-clones exactly do exist? Are there only countably many $C$-clones?

To show that there are only countably many $C$-clones, one could try to show that for all $\operatorname{Pol} Q$, where $Q$ is a subset of clausal relations, there is already a finite set $Q_{0} \subseteq C \operatorname{Inv} \operatorname{Pol} Q$ such that

$$
\operatorname{Pol} Q=\operatorname{Pol} Q_{0}
$$

Below we show that the latter statement implies $\left|\mathcal{C} \mathcal{L}_{D}\right|=\aleph_{0}$. First, we show that if $|D| \leq \aleph_{0}$, then $\left|C \mathrm{R}_{D}\right| \leq \aleph_{0}$.

Let $p, q \in \mathbb{N}_{+}$. Consider the set $\mathcal{R}_{q}^{p}=\left\{\mathrm{R}_{\mathbf{b}}^{\mathbf{a}} \mid(\mathbf{a}, \mathbf{b}) \in D^{p} \times D^{q}\right\}$ of all clausal relations of arity $p+q$. The function $\varphi: D^{p} \times D^{q} \longrightarrow \mathcal{R}_{q}^{p}$ defined by

$$
\varphi(\mathbf{a}, \mathbf{b}):=\mathrm{R}_{\mathbf{b}}^{\mathbf{a}}
$$

is surjective. This implies $\left|\mathcal{R}_{q}^{p}\right| \leq\left|D^{p} \times D^{q}\right|=\left|D^{p+q}\right|$. Since $|D| \leq \aleph_{0}$, we have $\left|D^{p+q}\right| \leq \aleph_{0}$ and hence, $\left|\mathcal{R}_{q}^{p}\right| \leq \aleph_{0}$. Thus,

$$
\left|C \mathrm{R}_{D}\right|=\left|\bigcup_{(p, q) \in \mathbb{N}_{+}^{2}} \mathcal{R}_{q}^{p}\right| \leq \aleph_{0} .
$$

Second, let $\mathcal{P}_{\text {fin }}\left(C \mathrm{R}_{D}\right)$ be the set of all finite subsets of $C \mathrm{R}_{D}$. Because $\left|C \mathrm{R}_{D}\right| \leq \aleph_{0}$, we have $\left|\mathcal{P}_{\text {fin }}\left(C \mathrm{R}_{D}\right)\right| \leq \aleph_{0}$. Finally, consider the mapping

$$
\begin{aligned}
\psi: \mathcal{P}_{\text {fin }}\left(C \mathrm{R}_{D}\right) & \longrightarrow \mathcal{C} \mathcal{L}_{D} \\
Q_{0} & \mapsto \operatorname{Pol} Q_{0} .
\end{aligned}
$$

If we can prove that for all $\operatorname{Pol} Q$, where $Q$ is a subset of clausal relations, there is already a finite set $Q_{0} \subseteq C \operatorname{Inv} \operatorname{Pol} Q$ such that

$$
\operatorname{Pol} Q=\operatorname{Pol} Q_{0}
$$

we get that $\psi$ is surjective and hence,

$$
\left|\mathcal{C} \mathcal{L}_{D}\right| \leq\left|\mathcal{P}_{\text {fin }}\left(C \mathrm{R}_{D}\right)\right| \leq \aleph_{0}
$$


P2. Is there an uncountable set of $C$-clones? Is there a continuum of $C$-clones on a set containing at least three elements?

To show that there is a continuum of $C$-clones one could try to show the following: Let $F$ be a suitably chosen countable set of functions on $D$. Then, for all $f \in F$ one had to prove $f \notin \operatorname{Pol} C \operatorname{Inv}(F \backslash\{f\})=\langle F \backslash\{f\}\rangle_{\mathcal{C}}$.

In the following remark, we show that the latter statement implies $\left|\mathcal{C} \mathcal{L}_{D}\right|=2^{\aleph_{0}}$. In [VPT97] there is a form to prove that there is a continuum of clones on finite sets $D$.

7.0.5 Remark. Let $F$ be a countable set of functions on $D$ such that for all $f \in F$ holds $f \notin\langle F \backslash\{f\}\rangle_{\mathcal{C}}$. Then for all $G, H$ subsets of $F$ such that $G \neq H$ we get $\langle G\rangle_{\mathcal{C}} \neq\langle H\rangle_{\mathcal{C}}$.

Proof. Let $F \subseteq \mathrm{O}_{D}$ be countable, such that $f \notin\langle F \backslash\{f\}\rangle_{\mathcal{C}}$ holds for all $f \in F$, and let $G, H \subseteq F$, where $G \neq H$. The latter implies $G \nsubseteq H$ or $H \nsubseteq G$. W.l.o.g. $G \nsubseteq H$. Hence, there is some $g \in G$ such that $g \notin H$. Since $g \notin H$, we get $H \subseteq F \backslash\{g\}$ and $\langle H\rangle_{\mathcal{C}} \subseteq\langle F \backslash\{g\}\rangle_{\mathcal{C}}$. Since $g \notin\langle F \backslash\{g\}\rangle_{\mathcal{C}}$, $g \notin\langle H\rangle_{\mathcal{C}}$ follows. Furthermore, as $g \in\langle G\rangle_{\mathcal{C}}$, we get $\langle G\rangle_{\mathcal{C}} \nsubseteq\langle H\rangle_{\mathcal{C}}$. Hence, $\langle G\rangle_{\mathcal{C}} \neq\langle H\rangle_{\mathcal{C}}$.

Let $F$ be a countable set of functions on $D$ as above. From the previous remark, the following mapping

$$
\begin{aligned}
\varphi: \mathcal{P}(F) & \longrightarrow \mathcal{C} \mathcal{L}_{D} \\
G & \mapsto\langle G\rangle_{\mathcal{C}}
\end{aligned}
$$

is injective. Hence,

$$
2^{\aleph_{0}}=|\mathcal{P}(F)| \leq\left|\mathcal{C} \mathcal{L}_{D}\right| \leq\left|\mathcal{L}_{D}\right|=2^{\aleph_{0}}
$$

P3. In Chapter 5 we determined all atoms in the lattice of essentially unary $C$-clones. A problem for future research is to determine all atoms in the lattice of $C$-clones.

P4. The following open problem is related to the characterization of the Galois closed sets for Pol $-C$ Inv.

From the restriction of the Galois connection Pol - Inv to the Galois connection $\mathrm{Pol}-C$ Inv, we get $\operatorname{Pol} \operatorname{Inv}(F)=\langle F\rangle_{O_{D}} \subseteq\langle F\rangle_{\mathcal{C}}$, where $F$ is a set of operations on $D$. The question is to determine the new functions in

$$
\langle F\rangle_{\mathcal{C}} \backslash\langle F\rangle_{O_{D}}
$$

in a constructive way that is essentially different from applying Pol $C$ Inv.

P5. Answer the open question at the end of Chapter 5, related to the characterization of the Galois closed sets for End - $C$ Inv. 
P6. Answer the open questions at the end of Section 6.2, related to sets of unary functions that are $C$-complete.

F.R. Future research:

In what follows, we mention a result on the order of certain one-generated $C$-clones. Although the proof of Theorem 7.0.6 below will be shown in [BV11], we consider it convenient to mention the theorem, and an example to illustrate it. First, we introduce some notation.

Notation: Let $p, q \in \mathbb{N}_{+}, \mathbf{a}=\left(a_{1}, \ldots, a_{p}\right) \in D^{p}$ and $\mathbf{b}=\left(b_{1}, \ldots, b_{q}\right) \in D^{q}$. For $I \subseteq\{1, \ldots, p\}$ let $a_{\wedge I}:=\min \left\{a_{v} \mid v \in I\right\}$, likewise $b_{\vee J}:=\max \left\{b_{v} \mid v \in J\right\}$ for $J \subseteq\{1, \ldots, q\}$.

Let $p, q, R_{a}, R_{b} \in \mathbb{N}_{+}, \mathbf{a}=\left(a_{1}, \ldots, a_{p}\right) \in D^{p}, \mathbf{b}=\left(b_{1}, \ldots, b_{q}\right) \in D^{q}$. Let $\left\{I_{1}^{a}, \ldots, I_{R_{a}}^{a}\right\} \subseteq \mathcal{P}(\{1, \ldots, p\}) \backslash\{\emptyset\}$ and $\left\{I_{1}^{b}, \ldots, I_{R_{b}}^{b}\right\} \subseteq \mathcal{P}(\{1, \ldots, q\}) \backslash\{\emptyset\}$ be collections of nonempty subsets of indices such that

(1) the sets $I_{1}^{a}, \ldots, I_{R_{a}}^{a}$ are pairwise different,

(2) $\bigcup_{t=1}^{R_{a}} I_{t}^{a}=\{1, \ldots, p\}$,

(3) there exist $k_{1}^{a}, \ldots, k_{R_{a}}^{a} \in\{1, \ldots, p\}$ pairwise different such that

$$
\forall t \in\left\{1, \ldots, R_{a}\right\}: k_{t}^{a} \in I_{t}^{a} \text { and } a_{\wedge I_{t}^{a}}=a_{k_{t}^{a}} ;
$$

(1') the sets $I_{1}^{b}, \ldots, I_{R_{b}}^{b}$ are pairwise different,

(2') $\bigcup_{s=1}^{R_{b}} I_{s}^{b}=\{1, \ldots, q\}$,

(3') there exist $k_{1}^{b}, \ldots, k_{R_{b}}^{b} \in\{1, \ldots, q\}$ pairwise different such that

$$
\forall s \in\left\{1, \ldots, R_{b}\right\}: k_{s}^{b} \in I_{s}^{b} \text { and } b_{\vee I_{s}^{b}}=b_{k_{s}^{b}} .
$$

Note that these conditions are trivially true if $\left\{I_{1}^{a}, \ldots, I_{R_{a}}^{a}\right\}$ and $\left\{I_{1}^{b}, \ldots, I_{R_{b}}^{b}\right\}$ are partitions of $\{1, \ldots, p\}$ and $\{1, \ldots, q\}$, respectively.

7.0.6 Theorem ([BV11]). Let $\left\{I_{1}^{a}, \ldots, I_{R_{a}}^{a}\right\}$ and $\left\{I_{1}^{b}, \ldots, I_{R_{b}}^{b}\right\}$ be collections of nonempty subsets of indices satisfying the conditions mentioned above. Let also $\mathbf{c}:=\left(a_{\wedge I_{1}^{a}}, \ldots, a_{\wedge I_{R_{a}}^{a}}\right) \in D^{R_{a}}$, and $\mathbf{d}:=\left(b_{\vee I_{1}^{b}}, \ldots, b_{\vee I_{R_{b}}^{b}}\right) \in D^{R_{b}}$. Then

$$
\text { Pol R } \mathbf{b}_{\mathbf{b}}^{\mathbf{a}} \subseteq \operatorname{Pol} \mathrm{R}_{\mathbf{d}}^{\mathbf{c}}
$$

7.0.7 Example. Let $\mathbf{a}=(4,2,3,2), \mathbf{b}=(1,2,3,4,3)$. Consider, $R_{a}=2$, $R_{b}=3, \underline{I}_{1}^{a}=\{1,2,3\}, \underline{\sim}_{2}^{a}=\{2,4\}, \underline{I}_{1}^{b}=\{1,2,3\}, \underline{\sim}_{2}^{b}=\{2,3,4\}, I_{3}^{b}=\{2,3,5\}$, and the numbers $k_{1}^{a}=2, k_{2}^{a}=4, \bar{k}_{1}^{b}=3, k_{2}^{b}=4, k_{3}^{b}=5$ which satisfy the conditions (3) and (3') mentioned above. Then by 7.0.6, we get

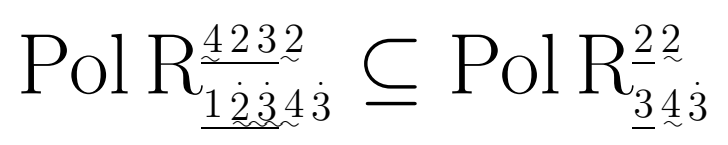





\section{Concluding remarks}

In this work we studied a special set of relations, so-called clausal relations, and we described clones that are determined by sets of these relations. These clones are called $C$-clones.

It was shown that clausal relations meet a sufficient condition that is known to ensure polynomial time solvability of the corresponding CSP, namely that their clone of polymorphisms contains a constant operation (see Section 3.4).

Our aim was the description of $C$-clones. First, the description of all $C$-clones on the two-element set was given (see Section 3.2). However, for $|D| \geq 3$ the description of $C$-clones has been proven to be a lot more difficult, due to the infinitely many $C$-clones showed in Section 3.5, by constructing infinite descending chains of these clones. Therefore, we analysed the $C$-clones up to equality of their unary bijective parts, i.e. $C$-automorphism groups.

The main result in the description of all $C$-automorphism groups is that they form a Boolean lattice with $|D|-1$ atoms (see Section 4.1). In particular, the $C$-automorphism groups can be characterised by permutations preserving some of their unary relations, which are intervals w.r.t. the natural order on $D$.

Furthermore, we studied the $C$-clone lattice up to equality of the corresponding endomorphism monoids, which is the lattice of $C$-monoids. The structure of this lattice is considerably harder to understand, since unary relations are not any more enough to determine the lattice of all relational clones given by $C$-monoids. However, first steps towards the description of this lattice were undertaken. First, it was shown that finite sets of clausal relations suffice to describe $C$-monoids (see Section 5.1). Then, the lattice of $C$-monoids for $|D|=3$ was determined (see Section 5.2). After that, the co-atoms and the atoms of the $C$-monoid lattice were characterised (see Sections 5.3 and 5.4).

Finally, all co-atoms in the lattice of $C$-clones were determined. It turned out that one non-trivial clausal relation of arity two can be used to describe both a co-atom in the lattice of $C$-clones and a co-atom in the lattice of $C$-monoids (see Section 6.1 ).

In future investigations, we will study the structure of the $C$-clone lattice on a finite set $D$. 



\section{Notation}

$f\left[\mathbf{r}_{1}, \ldots, \mathbf{r}_{k}\right] \ldots \ldots \ldots$ composition of $f \in \mathrm{O}_{D}^{(k)}$ by tuples $\mathbf{r}_{1}, \ldots, \mathbf{r}_{k} \in D^{m}$ $m \in \mathbb{N}_{+}$

$f\left(g_{1}, \ldots, g_{k}\right) \ldots \ldots \ldots$ general composition of $f \in \mathrm{O}_{D}^{(k)}$ with $g_{1}, \ldots, g_{k} \in \mathrm{O}_{D}^{(m)}$

$|D| \ldots \ldots \ldots \ldots \ldots \ldots$ cardinality of a set $D$

Aut $-C \operatorname{Inv} \ldots \ldots \ldots$ Galois connection Aut $-C$ Inv

Aut - Inv ......... Galois connection Aut - Inv

Aut $Q \ldots \ldots \ldots$ set of weak automorphisms of a set $Q$ of finitary relations

$c_{a} \ldots \ldots \ldots \ldots \ldots \ldots$ constant unary function with value $a$

$c_{a}^{k} \ldots \ldots \ldots \ldots \ldots$ constant $k$-ary function with value $a$

$C \operatorname{Inv} F \ldots \ldots \ldots$ set of invariant clausal realtions relations of a set $F$ of finitary operations

$\mathcal{C} \mathcal{L}_{D} \ldots \ldots \ldots \ldots$ lattice of all $C$-clones on a set $D$

$\mathcal{C} \mathcal{L}_{D}^{*} \ldots \ldots \ldots \ldots \ldots$ lattice of all relational $C$-clones on a set $D$

$\langle F\rangle_{\mathcal{C}} \ldots \ldots \ldots \ldots \ldots, C$-clone of operations (Pol $C$ Inv $F$ ) of a set $F$ of finitary operations

$\langle F\rangle_{\mathcal{C} O_{D}^{(1)}} \ldots \ldots \ldots \ldots \ldots, C$-monoid of operations (End $C \operatorname{Inv} F$ ) of a set $F$ of unary operations

$C \mathrm{R}_{D} \ldots \ldots \ldots \ldots \ldots$ set of all finitary clausal relations on a set $D$

$[Q]_{\mathcal{C}} \ldots \ldots \ldots \ldots \ldots$ relational $C$-clone $(C \operatorname{Inv} \operatorname{Pol} Q)$ of a set $Q$ of finitary clausal relations

$C \mathrm{R}_{D}^{*} \quad \ldots \ldots \ldots \ldots \ldots$ set of non-trivial clausal relations on a set $D$

CSP $\ldots \ldots \ldots \ldots \ldots$ Constraint Satisfaction Problem

$[Q]_{w \mathcal{C}} \ldots \ldots \ldots \ldots \ldots$ weak Krasner $C$-clone $(C \operatorname{Inv}$ End $Q)$ of a set $Q$ of finitary clausal relations 
$\Delta_{D}^{(2)}$

$\operatorname{diag}(D)$

D

$\mathrm{R}_{\mathbf{a}^{\alpha}}^{\mathbf{b}^{\alpha}}$

$\emptyset$

End $-C$ Inv

End - Inv

End $Q$

$e_{i}^{(k)}$

$\langle F\rangle_{O_{D}}$

$F \leq \mathrm{O}_{D}$

$f \triangleright \varrho$

$i d_{D}$

$\operatorname{Im}(f)$

$\operatorname{Int} \mathrm{R}_{\mathrm{b}}^{\mathrm{a}}$

$\operatorname{Inv} F$

$\operatorname{Inv}^{(m)} F$

$J_{D}$

$\mathcal{L}_{D}$

$\mathcal{L}_{D}^{*}$

$\nabla_{D}^{(2)}$

$\mathbb{N}_{+}$

$\mathbb{N}_{\geq 2}$

$\mathrm{O}_{D}$

$\mathrm{O}_{D}^{(k)}$ binary identity relation on $D$

set of all diagonal relations together with the empty relation

finite set $\{0,1, \ldots, n-1\}$

dual clausal relation of arity $p+q$ on a set $D$

emptyset

Galois connection End - $C$ Inv

Galois connection End - Inv

set of endomorphisms of a set $Q$ of finitary relations

$k$-ary projection on the $i$-th coordinate $(i \in\{1, \ldots, k\})$

clone generated by $F \subseteq \mathrm{O}_{D}$

$F \subseteq \mathrm{O}_{D}$ is a clone on $D$

. finitary operation $f$ preserves a finitary relation $\varrho$

identity mapping on a set $D$

image $f[A]$ of a function $f: A \longrightarrow B$

intervals belonging to $\mathrm{R}_{\mathrm{b}}^{\mathrm{a}}$

set of invariant relations of a set $F$ of finitary operations

set of $m$-ary relations in $\operatorname{Inv} F$

set of all projections on a set $D$

lattice of all clones on a set $D$

lattice of all relational clones on a set $D$

full binary relation on $D, D \times D$

the set of positive natural numbers

the set of natural numbers greater or equal to 2

set of all finitary operations on a set $D$

set of all $k$-ary operations on a set $D$ 
Pol $-C$ Inv $\ldots \ldots \ldots$ Galois connection Pol $-C$ Inv

Pol - Inv ......... Galois connection Pol - Inv

$\mathrm{Pol}^{(k)} Q \ldots \ldots \ldots \ldots$ set of $k$-ary operations in $\operatorname{Pol} Q$

Pol $Q \ldots \ldots \ldots \ldots \ldots$ set of polymorphisms of a set $Q$ of finitary relations

$\mathcal{P}(D) \ldots \ldots \ldots \ldots \ldots$ powerset of $D$

$\operatorname{pr}_{i}\left(\mathrm{R}_{\mathbf{b}}^{\mathbf{a}}\right)^{C} \ldots \ldots \ldots \ldots$ projection on the $i$-th coordinate $(i \in\{1, \ldots, p\})$ of $\left(\mathrm{R}_{\mathbf{b}}^{\mathbf{a}}\right)^{C}$

$[Q]_{\mathrm{R}_{D}} \cdots \cdots \cdots \cdots \cdots$ relational clone generated by $Q \subseteq \mathrm{R}_{D}$

$Q \leq \mathrm{R}_{D} \ldots \ldots \ldots \ldots, Q \subseteq \mathrm{R}_{D}$ is a relational clone on $D$

$\mathrm{R}_{D} \ldots \ldots \ldots \ldots$ set of all finitary relations on a set $D$

$\mathrm{R}_{b}^{a} \quad \ldots \ldots \ldots \ldots \ldots$ binary clausal relation on $D$

$\mathrm{R}_{\mathbf{b}}^{\mathbf{a}} \ldots \ldots \ldots \ldots \ldots \ldots$ clausal relation of arity $p+q$ on a set $D$

$\left(\mathrm{R}_{\mathbf{b}}^{\mathbf{a}}\right)^{C} \ldots \ldots \ldots \ldots \ldots$ complement of a clausal relation of arity $p+q$ on a set $D$

$\mathrm{R}_{\mathbf{0}, q}^{\mathbf{1}, p} \ldots \ldots \ldots \ldots \ldots$ clausal relation of arity $p+q$ on the two-element set

$\mathrm{R}_{D}^{(m)} \ldots \ldots \ldots \ldots \ldots$ set of all $m$-ary relations on a set $D$

$\mathrm{S}_{D} \ldots \ldots \ldots \ldots \ldots$ set of all permutations on a set $D$

$V_{\alpha} \ldots \ldots \ldots \ldots \ldots$ contravariant substitution functor applied to a mapping $\alpha: m \longrightarrow n, m, n \in \mathbb{N}_{+}$

$W_{\alpha} \ldots \ldots \ldots \ldots \ldots$ covariant substitution functor applied to a mapping $\alpha: m \longrightarrow n, m, n \in \mathbb{N}_{+}$ 



\section{Index}

$C$-clone, 17

smallest Boolean, 22

$C$-clones

Boolean, 18

$C$-automorphism group, 47

$C$-clone

maximal, 85

$C$-monoid, 57

maximal, 66

minimal, 69

automorphism, 7

clausal relation, 11

finitary, 11

inclusion, 16

covariant substitution

functor, 13

identification of coordinates, 14

intersection, 12

intervals belonging to, 49

non-trivial, 15

permutation, 13

union, 12

clone, 1

generated, 2

full, 2

monotone Boolean

functions, 3

trivial, 2

closure operator, 5

composition

of functions, 1

Constraint Satisfaction Problem, 10 cover, 18

dual

clausal relation, 31

operation, 29

endomorphism, 7

Galois closed sets, 6

Galois connection, 6

Aut - $C$ Inv, 47

End $-C$ Inv, 57

Pol $-C$ Inv, 17

Pol - Inv, 7

Krasner $C$-clone, 47

weak, 57

Krasner clones, 9

lattice

of partitions on $D, 54$

of $C$-automorphism group, 50

of some $C$-monoids, 77

of Boolean $C$-clones, 26

operation

finitary, 1

median, 20

constant, 2

monotone, 2

projection, 1

operation

ternary minority, 24

operations on relations

contravariant substitution

functor, 4 
Index

covariant substitution

functor, 4

identification of coordinates, 4

intersection, 4

permutation of coordinates, 4

projections onto coordinates, 5

polymorphism, 6

relation

finitary, 3

identity, 3

invariant, 6

relational

$C$-clone, 17

clone, 3

clone generated, 5

symmetric group, 4, 7

weak-Krasner clones, 9 


\section{References}

[Beh09] Mike Behrisch. Relational tame congruence theory and subalgebra primal algebras. Diplomarbeit, TU Dresden, 2009.

[BKJ00] Andrei A. Bulatov, Andrei A. Krokhin, and Peter Jeavons. Constraint satisfaction problems and finite algebras. In Automata, languages and programming (Geneva, 2000), volume 1853 of Lecture Notes in Comput. Sci., pages 272-282. Springer, Berlin, 2000.

[Bul02] Andrei A. Bulatov. A dichotomy theorem for constraints on a threeelement set. In FOCS, pages 649-658. IEEE Computer Society, 2002.

[BV10] Mike Behrisch and Edith M. Vargas. $C$-clones and $C$-automorphism groups. In Contributions to general algebra. 19. Proceedings of the Olomouc Workshop 2010 on General Algebra., pages 1-12. Verlag Johannes Heyn, Klagenfurt, 2010.

[BV11] Mike Behrisch and Edith M. Vargas. On the $C$-clone lattice. In preparation, 2011.

[CHKS08] Nadia Creignou, Miki Hermann, Andrei Krokhin, and Gernot Salzer. Complexity of clausal constraints over chains. Theory Comput. Syst., 42(2):239-255, 2008.

[DP02] B. A. Davey and H. A. Priestley. Introduction to lattices and order. Cambridge University Press, New York, second edition, 2002.

[DW02] Klaus Denecke and Shelly L. Wismath. Universal algebra and applications in theoretical computer science. Chapman \& Hall/CRC, Boca Raton, FL, 2002.

[GW99] Bernhard Ganter and Rudolf Wille. Formal concept analysis. SpringerVerlag, Berlin, 1999. Mathematical foundations, Translated from the 1996 German original by Cornelia Franzke.

[JCG97] Peter Jeavons, David Cohen, and Marc Gyssens. Closure properties of constraints. J. ACM, 44(4):527-548, 1997.

[Jea98] Peter Jeavons. On the algebraic structure of combinatorial problems. Theoret. Comput. Sci., 200(1-2):185-204, 1998. 
[PK79] R. Pöschel and L. A. Kalužnin. Funktionen- und Relationenalgebren, volume 15 of Mathematische Monographien [Mathematical Monographs]. VEB Deutscher Verlag der Wissenschaften, Berlin, 1979. Ein Kapitel der diskreten Mathematik. [A chapter in discrete mathematics].

[Pos41] Emil L. Post. The Two-Valued Iterative Systems of Mathematical Logic. Annals of Mathematics Studies, no. 5. Princeton University Press, Princeton, N. J., 1941.

[Pös80] Reinhard Pöschel. A general Galois theory for operations and relations and concrete characterization of related algebraic structures, volume 1 of Report 1980. Akademie der Wissenschaften der DDR Institut für Mathematik, Berlin, 1980. With German and Russian summaries.

[Pös04] R. Pöschel. Galois connections for operations and relations. In Galois connections and applications, volume 565, pages 231-258. 2004.

[Var10] Edith Vargas. Clausal relations and C-clones. Discuss. Math. Gen. Algebra Appl., 30(2):147-171, 2010.

[Var12] Edith M. Vargas. Maximal and minimal $C$-monoids. Demonstratio Math., 2012. to appear.

[VPT97] Gradimir Vojvodić, Jovanka Pantović, and Ratko Tošic. The number of clones containing an unary function. Novi Sad J. Math., 27(2):83-87, 1997. 


\section{Affirmation}

(a) Hereby I declare that I wrote the present thesis on my own, I indicated citations. I did not use any other means than indicated. This thesis has not been presented to any other examination board in this or a similar form, neither in Germany nor in any other country.

(b) The present thesis has been produced since April 2007 at the Institut für Algebra, Department of Mathematics, Faculty of Science, TU Dresden under the supervision of Prof. Dr. rer. nat. habil. R. Pöschel.

(c) There have been no prior attempts to obtain a $\mathrm{PhD}$ at any university.

(d) I accept the requirements for obtaining a $\mathrm{PhD}$ (Promotionsordnung) of the Faculty of Science of the TU Dresden, issued March 20, 2000 with the changes in effect since April 16, 2003 and July 17, 2008.

\section{Erklärung}

(a) Hiermit erkläre ich, dass ich die vorliegende Arbeit selbständig erarbeitet, verfaßt und Zitate kenntlich gemacht habe. Andere als die angegebenen Hilfsmittel wurden von mir nicht benutzt. Die Arbeit wurde bisher weder im Inland noch im Ausland in gleicher oder ähnlicher Form einer anderen Prüfungsbehörde vorgelegt.

(b) Die vorliegende Dissertation wurde im April 2007 am Institut für Algebra, Fachrichtung Mathematik, Fakultät Mathematik und Naturwissenschaften, Technische Universität Dresden unter der Betreuung von Prof. Dr. rer. nat. habil. R. Pöschel. angefertigt.

(c) Es wurden zuvor keine Promotionsvorhaben unternommen.

(d) Ich erkenne die Promotionsordnung der Fakultät Mathematik und Naturwissenschaften der TU Dresden vom 20. März 2000, in der geänderten Fassung mit Gültigkeit vom 16. April 2003 und vom 17. Juli 2008 an.

Dresden, den 25. Februar 2011 